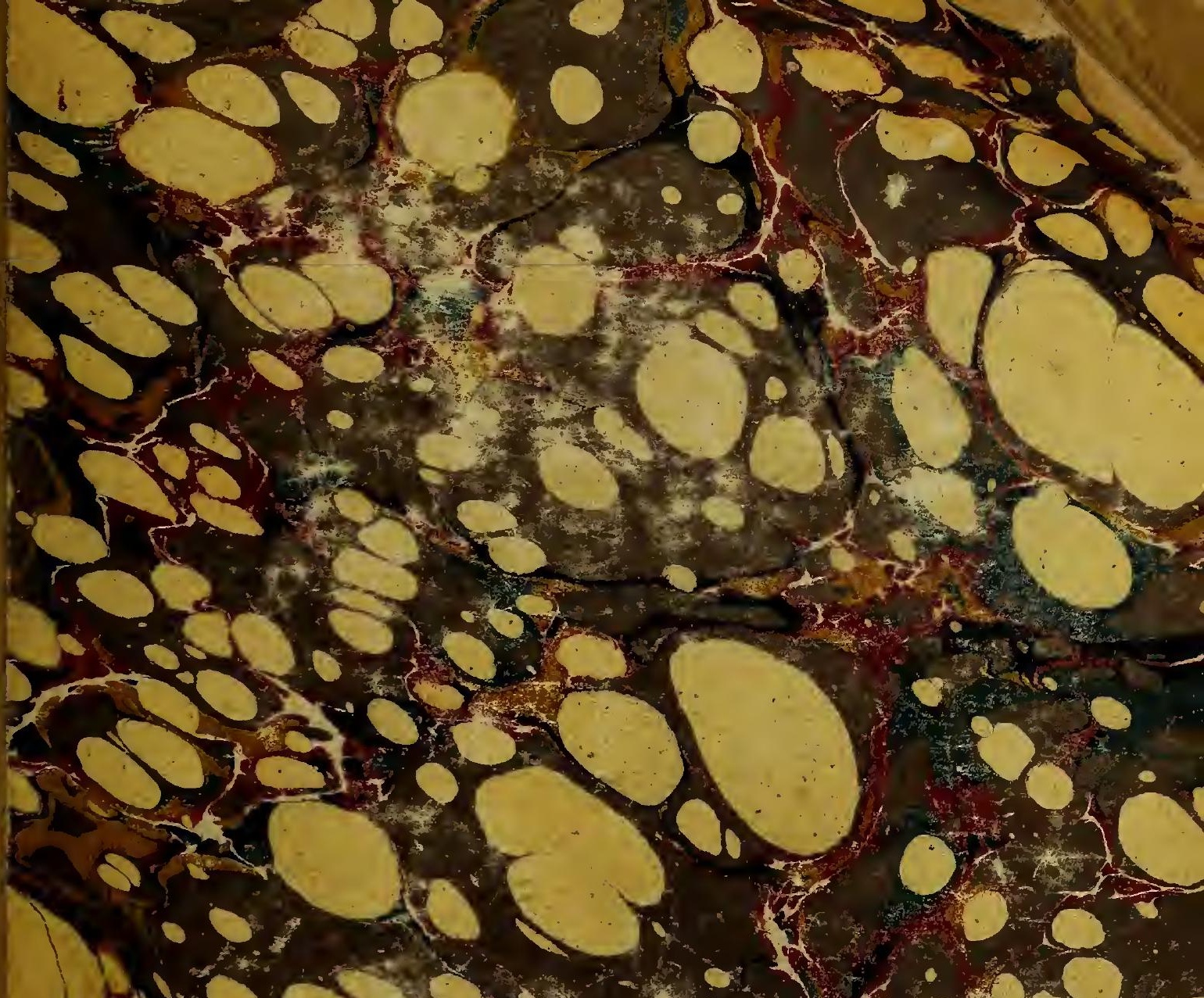

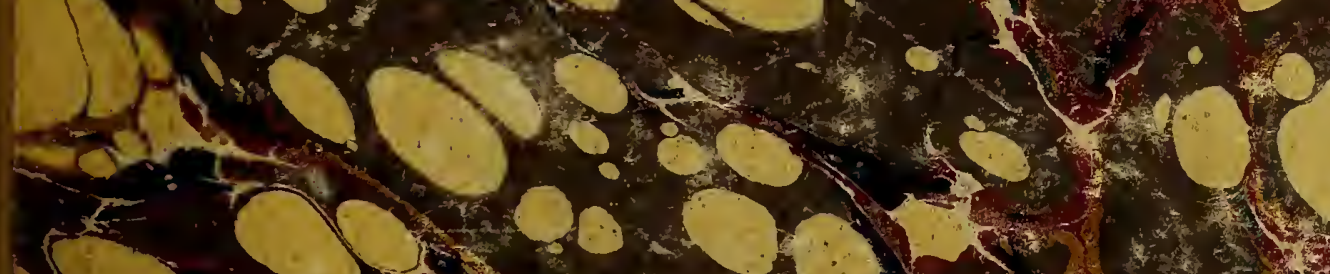

$580^{0}, 0 \%$

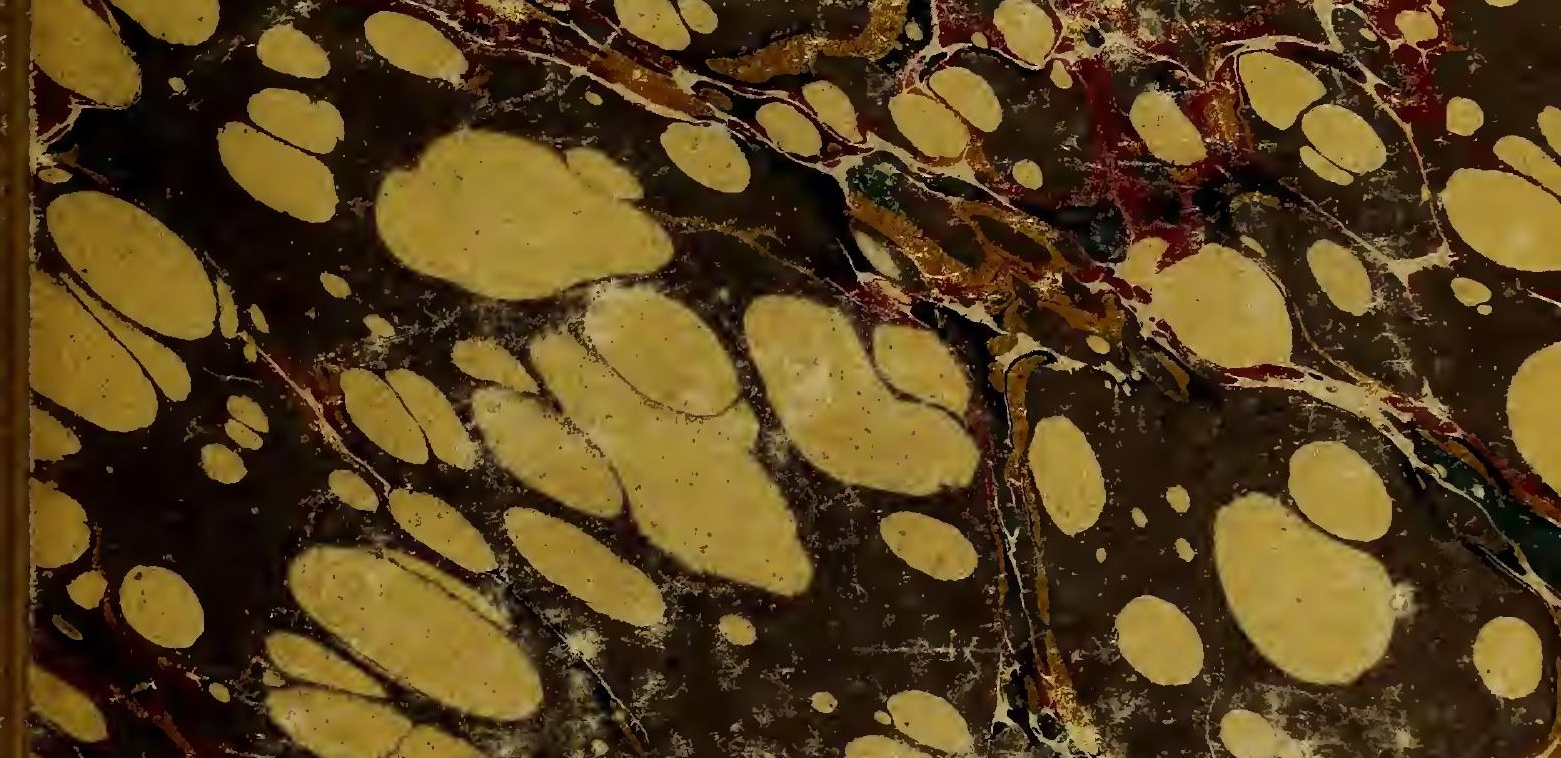

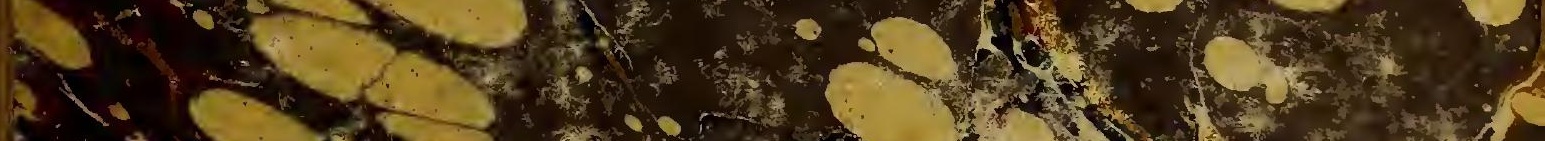

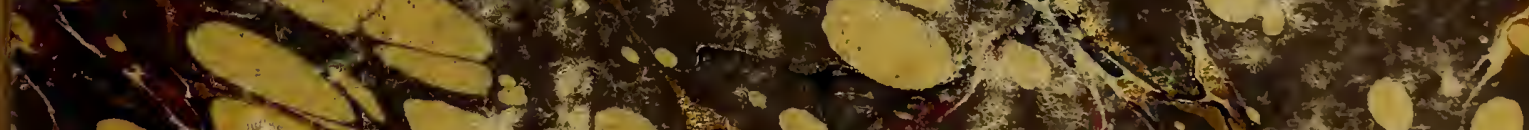

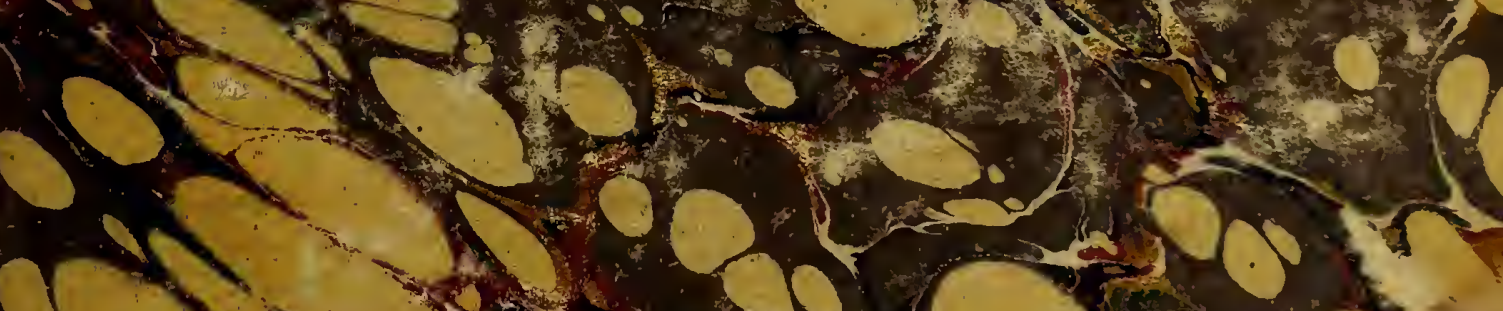

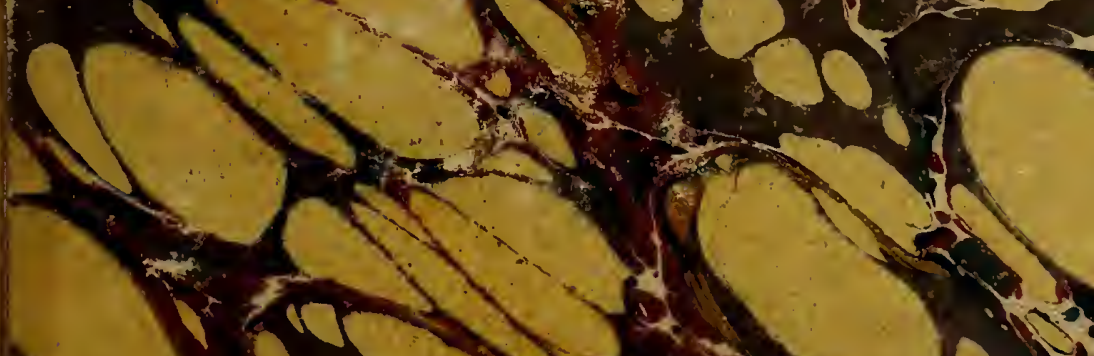


5
0
oracts. 
1 felbys arrangement of Birds

2 Bonapartes Conspectus syotemates ornithologie 3 "Conspectus of Burds of Prey

4 "Monograph of Laniines

5 Boureir \& mulsant on Irochilus Stanlegi

6 Lafresnaye on Dendrocolaptince te.

7 Moores Monograph of Ruticilla

8 "1 on tome N. Sidian Bros

9 "on ppecies of Orthotomus

10 Sclater Ianagrarum Catalogus specifiens

11 Bonaparte on Larides

12 Ideuglin on Birds of $\mathcal{A}$. East african

13. Sale Catalogue of Boris of Prince D'Essling. 



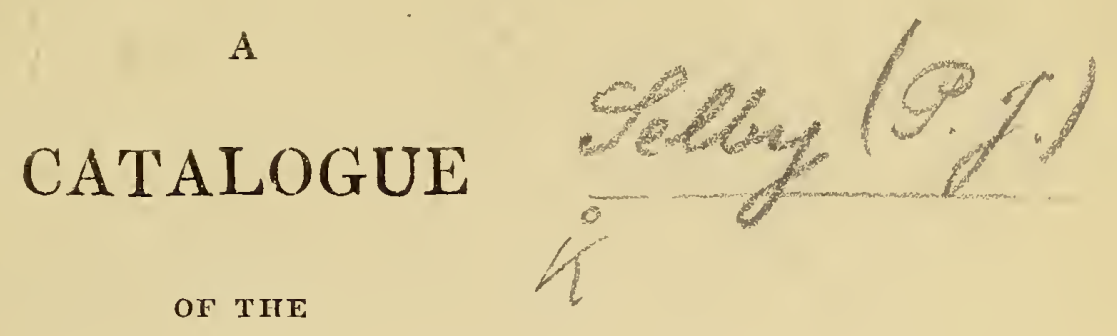

GENERIC AND SUB-GENERIC TYPES

OF THE CLASS

\section{AVES, BIRDS,}

ARRANGED ACCORDING TO THE NATURAL SYSTEM;

WITH

SEPARATE LISTS,

DISTINGUISHING THE

VARIOUS QUARTERS OF THE GLOBE

IN WHICH

THEY ARE TO BE PROCURED.

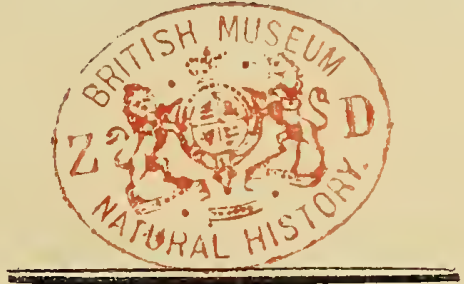

NEWCASTLE :

PRINTED BY T. AND J. HODGSON, UNION-STREET.

1840 . 


$$
\begin{gathered}
6 \% \\
3-10 . \\
6 .
\end{gathered}
$$


The following arrangement was drawn up by P. J. Selby, Esq., at the request of some of the Committee of the Natural History Socierty, and is printed for the purpose of exhibiting the Genera wanting in their collection, and to facilitate the exchange of specimens.

Those Genera which they already have, are marked with a*. Specimens, or offers to exchange, will be thankfully received. 



\section{LIST, \&c.}

\section{Class. AVES.}

\section{ORdo I. RAPTORES.}

Fam. 1. VULTURID压. Vultures.

Genus Vultur, Linn.

Vul. fulvus. So. Africa.

Genus Cathartes, (1) ILL.

Cath. (Sarcoramphus, Dum.,) Condor. So. America.

Cath. Aura. Amer.

Genus Neophron, Sav.

Neop. Percnopterus. Europe and Africa.

Genus Gypaëtus. StorR.

* Gyp. barbatus. Europe and Africa.

Gyp. (Phene, Sav.) hypoleucus.(2)

FAM. II. FALCONID $\mathbb{E}$.

Subfan. I. AQUILINAE. Eagles.

Genus Aquila, Auct.(3)

* Aqu. Chrysaëtos. Britain.

* Aqu. (Haliæëtus, A uct.,) albicilla. Britain.

(1) The genus Cathartes, of Illiger, contains two distinct groups, or Subgenera, one of which ircludes the "King of the Vultures" (Sar. Papa), the Condor (Sar. Condor), \&cc., the other is represented by the Turkey Buzzard (Cath. Aura), of Amer., or the Cath. Uruba.

(2) As a subgeneric type, in all probability the Vultur hypoleucus, of the "Illustrations of Ornithology," No.3, will enter here.

(3) From Aquila should be separated as one subgeneric group, that of which $A$. albicilla, or $A$. lencocephala is typical. To this the title of Haliceettus has been given by various authors. Mr. Swainson's genus 
Genus Pandion, Sav.

* Pan. Haliæëtus. Britain, (1)

Pan. (Icthyaëtus, Horsf.) Horsfeldii. Java.

Genus Harpyia,(2) Cuv.

Har. destructor. So. America.

Genus Gypogeranus, ILL.

* Gyp. serpentarius. So. Africa.

Genus Circaëtus, Vieicl.

Cir. cinereus.

Subfam. CYMINDID $\approx$.

Genus Ibycter, VIeILL.

Ib. ater. America.

Genus Polyborus, Vieill.

Poly. Braziliensis. South America.

Genus Cymindis, Cuv.

Cy. cuculoïdes. America.

Genus Nauclerus, Vigors.

* Nau. furcatus. America.

Nau. (Elanus, SAv.) melanopterus. Africa.

Nau. (Gampsonyx(3), Vra.) Swainsonii. America.

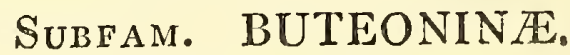

Genus Milvus, Auct.

* Mil. regalis. Britain.

Genus Pernis, Cuv.

* Per. apivorus. Britain.

Genus Spizaëtus, Vieılı.

Spi. cristatellus. India, \& c .

IIalixütus comprehends a different group-to which the name of $H a_{-}$ liastur is now given.

(1) As a subgeneric group, that of which the F. Icthyaëlus of Hors. is a type, here takes its station.

(2) It is probable that the genus IIomatornis of Gould, will enter among the Harpies, or form a subgeneric group of that genus.

(3) The genus Nauclerus of Vigors, appears to contain three distinct groups, or subgenera, viz., Nauclerus, Elanus, and Gampsonyx. 
Genus Buteo, Antra.

* But. vulgaris. Britain.

Genus Circus, Bechst.

* Cir. cyaneus. Britain.

SubFam. FALCONINA:.

Genus Falco,(1) A Uст.

* Fal. peregrinus. Britain.

Genus Harpagus, Vigors.

Har. corulescens. India, Ceylon, \& c.

Genus Lepidogenys,(2) Gould.

Lep. Indicus. India.

Genus Aviceda, Swain.

Av. cuculoïdes. West Africa.

\section{SubFam, ACCIPITRINÆE.}

Genus Ictinia, Vieill.

Ic. plumbea. America.

Genus Accipiter, Wil L.

* Ac. Nisus. Britain.

Genus Astur, Auct.

* As. palumbarius. Britain.

Genus Haliastur,(3) Mırr.

Hal. Pondecerianus. India.

(1) It is probable that the genus Ieracidea of Gould, will only prove to be an aberrant, or subgeneric form, of the restricted genus Falco, or of the same value as the group of the Kestrils, which, by some, have been separated from Faleo.

(2) A second species of this genus is given in Gould's "Synopsis of the Birds of Australia," under the title of Lepidogenys subcristatus, Lepidogenys having been substituted for Lophotes, as that term had been previously employed.

(3) Whether this group, of which F. Pondecerianus Auct. is a type, belongs to this subfam., or to the Pandions (Ospreys), seems a matter of doubt. I have substituted the title of Haliastur for that of Haliceertus, given it by Mr. Swainson, as the latter already designates a group of the Eagles. To it will belong the Haliceettus leucosternus and Halixeetus canorus of "Gould's Syn. of the Birds of Australia." 
FAM. STRIGID $\mathbb{E}$.

Genus Strix,(1) Linn.

* Str. flammea. Britain.

* Str. (Ulula, Ser b; Scotiaptex, Swa In.) Stridula. Brit.

* Str. (Scotophilus, Swarn.) Tengmalmi. Br. and Europe.

* Str. (Otus, Auct.) vulgaris. Britain.

Genus Bubo,(2) Cuvier.

* Bu. maximus. Brituin and Europe.

Bu. (Heliaptex, Swarn) arcticus. Arc., America.

* Bu. (Scops, Antiq.) Aldrovandi. Br. and Europe.

Genus Nyctea, SAvig.

* Nyc. candida. Britain and Arctic Regions.

Genus Nyctipetes, Swain.

* Nyc. nudipes. Britain and Europe.

Genus Surnia,(3) Dumer.

* Sur, funerea. America, and N. Europe.

\section{ORDO II. INSESSORES, VI\%。}

Trtbe. DENTIROSTRES.

FAM. LANIADZE.

Subfam. IANIAN 2 .

Genus Lanius, LinN.

* Lan. Excubitor. Britain.

(1) The subfamilies of the Owls have not yet been recognized, but it is likely that the genera already established, will, when the species become better known, and the groups properly analysed, assume that rank. According to Mr. Swainson's views, the genus Strix contains four subgeneric, or, perhaps, they may form generic groups, viz., Strix, Ulula, Scoloplitus, and Otus.

(2) Under the genus $B u b o$ we have, at least, three well-characterized groups.

(3) Under this genus, or", as a subgeneric form, it is probable the Athene fortis and Ali. Strenu of "Gould's Birds of Australia," will take their place. 'The Alticne maculata and At7. Boobook, of the same author, appear to belong to the genus Nyctipetes, Swain. 
Genus Telophonus, Swain.

Tel. leucogrammicus. Africa.

Genus Chætoblemma, Swain.

Chæ. leucocephala. Africa.

Genus Nilaus, Swain.

Nil. Capensis. Africa.

Genus Falcunculus, Vieill.

* Fal. frontalis. Australia.

Fal. (Cyclaris, Linn.) Guianensis. South America (1)

Subfam. THAMNOPHILIN $Æ$.

Genus Thamnophilus, Vieili.

Th. striatus. South America.

Genus Malaconotus, Swain.

Mal. atrococcineus. S'outh Africa.

Genus Prionops, Vieill.

Pri. plumatus. West Africa.

Genus Colluricincla, Vigors.

Col. cinerea. Australia.

SubFam. DICRURINÆE.

Genus Tephrodornis, SwaIN.

Teph. virgatus. Africa.

Genus Melasoma, Swain.

Mel. edolioïdes. West Africa.

Genus Ocypterus, Cuv.

Ocy. leucorynchus. India and Australia.

Genus Analcipus, Swain.

Anal. bicolur. Africa.

Genus Dicrurus, Vieill.

Dic. cristatus. India, \&c.

(1) By Mr. Swainson, Cyclaris Guianensis is now considered a Falcunculus, it appears, however, to be entilled to a subgeneric rank. 
SubFaM. CEBLEPYRIN E.

Genus Ceblepyris, Cuv.

Ceb. melanops. India and Australia.

Genus Oxynotus, Swain.

Oxy. ferrugineus. Indian Islands.

Genus Campephaga, VierLl.

Cam. atrata. Africa.

Genus Phœnicornis, Swain.

$\mathrm{Ph}$. flammeus. India.

Genus Erucivora, Swain.

Eru. orientalis. India.

SubFam. TYRANNINÆ.

Genus Ptilogonys, Swain.

Pti. cinereus. S. America.

Genus Chrysolophus, Swain.

Chr. ambulans. So. America.

Genus Saurophagus, Swain.

Sau. sulphuratus. America.

Genus Tyrannus, Vieill.

Ty. intrepidus. North America.

Ty. (Milvulus Swain.) forficatus.(1) America.

Genus Tyrannula, Swain.

Ty. audax. North America.

FAM. MERULID臣.

Surfam. BRACHYPODINE.

Genus Micropus, Swain.

Mic. calchocephalus, India.

Mic. (Hypsepetes Vrgors.) Psaroides.(2) India. Him.

(1) The long fork-tailed 'Tyrants have been designated by the subgeneric title of Milvulus.

(2) As a subgenus of Micropus, may at present be considered the G. Hypsepetes of "Vigors, of which lwo species are known. 
Genus Brachypus,(I) Swain.

Br. (Brachypus, Swain.) dispar. Africa.

Br. (chloropsis, JARd. and Selby, Cochinsinensis. India, or, Malabaricus. India.

Br. (Jora, Horsf.) scapularis. India, Java, \& c.

Br. (Andropadus, Swain.) vociferus. Africa.

Br. (Hæmatornis melanocephalus). India.

Genus Tricophorus, Tемм.

Tr. olivaceus. West Africa.

Genus Phyllastrephus, SwaIN.

Ph. Capensis. South Africa.

Genus Icteria, VI EILL.

Ic. polyglotta. North America.

SubFam. MYOTHERIN无.

Genus Dasycephala. Swarn.

Das. rufescens. South America.

Genus Myothera.(2) ILL.

My. capistrata. America.

My. (Drymophila, Swarn.) leucopus. So. America.

My. (Brachypteryx, Horsf.) montana. Java.

My. (Myocincla, Swain.) colma.

My. (Pithys, Vieill.) albifrons. America.

Genus Pitta,(3) Temm.

Pit. cyanura. India.

Pit. (Chlorisoma, Swain.) thalassina. India and China.

Pit. (Grallaria, Vieill.) Rex. India.

(1) The following five groups are considered as subgenera of Brachypus, viz.:-Brachypus, Swain.; Chloropsis, Jard. and Selby ; Jora Horsf.; Andropadus, Swain. and Hamatornis, Swain.

(2) In the genus Myothera of Illiger five groups or subgeneric forms are recognizable, viz. :-Myothera, Drymophila, Brachypteryx, Myocincla, and Pithys.

(3) Contains three subgeneric groups, viz.:-Pitta, Chlorisoma and Grallaria, in the last stands Chamoza meruloïdes of Vig. 
Genus Myophonus,(1) Temm.

My. metallicus. Asia and India.

Genus Cinclus, Bechst.

* Cin. aquaticus, Britain.

\section{Subfam. MERULINE.}

Genus Petrocincla, VIG.

Pet. saxatilis. Europe.

\section{Genus Merula,(2) RAY.}

* Merula nigra. Britain, and Merula viscivora. Brit. Genus Orpheus,(3) Swain.

Or. polyglottus. N. America.

Or. (Cussypha Vigors) leucoceps. West Africa. Genus Chætops, Swain.

Ch. Burchellii. Africa.

\section{Subfam. CRATEROPODIN若。}

\section{Genus Pellorneum, Swain.}

Pel. ruficeps. India.

Genus Crateropus, SwaIN.

Cr. Reinwardii. Africa.

$$
\text { Genus Grallina, VIEILL. }
$$

Gr. melanoleuca. Australia.

Genus Cinclosoma,(4) VIG. and Horsf.

Cinc. punctata. Australia.

(1) It seems not improbable that the Zoothera monticola of Gould" Contury will be found to be a subgenus of Myophonus.

(2) The one representing the dark, and the other the varied plumage of the Thrushes.

(3) Under this genus are placed the two subgeneric groups, Orpheus and Cossypha.

(4) Mr. Swainson considers G. Cinclosona, Vig. and Horsf., as a sub. genus of Grallina. 'This is doubtful, and it is here considered a generic form. 
Genus Malacocircus, Swain.

Mal. striatus. Africa.

Mal. (Megalurus, VIG. and Horsf.) palustris. India.

Mal. (Pomatorhinus, Horsf.) trivirgatus. India.

Mal. (Timalia, Horsf.) pileata. Java and India.

Genus Pteroptochus, (I) Kitslitz.

Pte. megapodius. South America.

SubFam。 ORIOLINAE.

Genus Donocobius, Swain.

Don. vociferans, America.

Genus Sericulus, SwaIn.

Se. chrysocephalus. Australia.

Genus Oriolus, Linn.

* Or. Galbula. Europe.

Or. (Irena, Horsf.) puella (2). Java.

Genus Dulus, Vieils.

Du. palmarum. South America.

Genus Sphecotheres, Vieili.

Sp. viridis. Australia.

FAM. SYLVIADE.

Subfam. SAXICOLINæ⿸(3)

Genus Gryllivora, Swain.

Gry. longicauda. Africa.

Genus Thamnobia, Swain.

Th. leucoptera. India.

Genus Saxicola, BECHST.

* Sax. Enanthe. Britain.

(1) This genus is inserted in the present sub-family on the autho. rity of Mr. Swainson.

(2) The Irena of Horsfield is now generally considered as a subgenus of Oriolus.

(3) By Gould, his genus or subgenus Sericomis is considered as belonging to the Saxicolinæ, as the type of it Seri. humilis or Seri. frontalis may be taker. Vide Gould's Syn. of Birds of Australia. 
Genus Erythaca. (1) SwalN.

* Ery. rubecula. Britain.

Ery. (Sialia, Swain.) Wilsonii. North America.

Genus Petroica, Swain.

* Pet. multicolor. Australia.

\section{Subfam. PHILOMELINE。}

Genus Phœnicura, Swain.

* Ph. ruticilla. Britain.

Genus Philomela, Auct.

* Phi. Luscinia. Britain.

* Phi. (Curruca, Bechst) hortensis. (2) Britain.

Genus Salicaria, Selby.

* Sal. phragmitis. Britain.

Genus Bradyptetus, Swain。

Brad. platyurus. Africa.

Genus Agrobates, SwaIn.

Ag. galactotes. Europe and Africa.

Subfam. SYLVIANR,

Genus Orthotomus, HorsF.

Or. longirostris. India.

Or. (Prinia, Honsf.) familiaris. India.

Genus Malurus, (3) Vieill.

Mal. (Hemipteryx, SwAIN.)

* Mal. (Drymoica, Swain.) cisticola, Europe.

* Mal. (Meluzophilus, Leach) provincialis. Britair.

* Mal. (Malurus, Vierll.) cyaneus. Australia.

Genus Sylvia, Latн.

* Sylv. Trochilus. Britain.

(1) 'Two subgeneric groups, Erythaea and Sialia. The latter com. prehending the Blue Birds of A merica. 'The former the'True Redbreasts.

(2) Curruca of Bechstein is here placed as a subgeneric group under Philomela; and Salicaria of Selby is considerea as a generic type.

(i) Under this genus four subgeneric groups have been recognized, viz.-Ncmipteryx, Drymoica, Mrelizoplihlus, and Malurus. 
Sylv. (Acanthiza, VIG and Horsf.) nana. Australia.

*

Sylv. (Regulus, RAx) auricapillus. Britain.

Sylv. (Cyanotis, Swain.) omnicolor. (1) So. America.

Genus Culicivora, Swain.

Cul. atricapilla. America.

Genus Praticola, Swa IN.

Pra. anthoides. Australia.

SUbFAm. PARIANÆ.

Genus Setophaga, SwaIN.

Set. ruticilla. North America.

Genus Sylvicola, (2) Swarn.

Sylv. (Dumecola, Swain.) ruficauda. North America.

Sylv. (Sylvicola, Swa rn.) pusilla. North America.

Sylv. (Vermivora, Swaln.) pinus. North America.

Sylv. (Mniotilta, Vieill.) varia. North America.

Sylv. (Zosterops, VIg. and Horsf.) dorsalis. Africa.

Genus Parus, (3) Linn.

Par. (Ægithina, Vieicl.) leucoptera. America.

Par. (Ægithalus, Vigors) pendulinus. Europe.

* Par. (Parus, Lin.) major. (4) Britain.

Par. (Parisoma, Swain.) rufiventer. Africa.

Par. (Hylophilus, Temm.) poicilotis. America.

(1) As subgeneric of Sylvia Mr. Swainson reckons Acanthiza of Vig. and Horsf., which appears to be the same with Gould's G. Sericor * nis; Regulus, Ray; and Cyanotis, Swain.

(2) Under this generic -head Swainson enumerates five subgeneric groups, viz.-Dumecola, Sylvicola, Vermivora, Mniotilta, and Zosterops.

(3) The five subgeneric groups of G. Parus are Agithina, Vieill.; EEgithalus, Vig.; Parus, Linn.; Parisoma, Swain.; and Hylophilus, Temm.

(4) The Bearded Titmouse is considered by Swainson as an aberrant species merely of the restricted genus Purus, representing the fissirostral type-by several late writers it has been elevated into a sub. generic type, under the title of Callimophilus. 
Genus Accentör, BECHST.

* Acc, modularis. Britain.

* Acc. (Seiurus, Swain.) auricapillus. (1) America.

Genus Trichas, Swain.

Tr. personata. North America.

Subfam. MOTACILLINE。

Genus Lessonia, Swain.

Les. erythronotus. South America.

Genus Motacilla, LINN.

* Mot. Yarrellii. (2) Britain.

* Mot. (Budytes, Cuv.) flava. Britain.

Genus Enicurus, Temm。

Eni. speciosus. Java.

Genus Anthus, Bechst。

* An. pratensis. Britain.

FAM. AMPELID压;

SubFam。LEIOTRICHAN在。

Genus Leiothrix, Swain.

Iei. furcatus. India.

Genus Pteruthius, Swain.

Pter. erythropterus. (3) India and Himalaya.

Subfam。VIREOIN E.

Genus Vireo, Vieill.

Vir. olivaceus. America.

(1) Seinrus of Swain. constitutes a subgenus of Accentor.

(2) $\Lambda$ s the Motacilla alba of English writers does not appear to be the true Moto alba of Limn. and continental authors, the name of Moto Yarrellii has been appropriately given to the British species. The true Mot, alba has not yet been met with in England. Mr. Swainson considers the Yellow Wagtails, or Budytes of Cuvier as forming a genexic group, but they appear rather to form a subgenus of Motacilla.

(3) This was first figured and described as a species of Lanius, by Gould, in the century of Himalayan Birds. Its true station, however, is undoubtedly in the present family. 
Genus Pachycephala, Swain.

Pach. gutturalis. Austr.

Pach. (Eopsaltria, Swa rn.) flavicollis. (1) Australia.

Genus Ptilichloris, Swain.

Pti. lunatus. Australia.

Genus Phibalura, Vifill.

Subfam. BOMBYCILLINÆ.

* Phi. cristata. South America.

Genus Bombycilla, Briss.

* Bom. garrula. Britain.

Genus Procnias, HofF.

Pro. ventralis. South America.

SubFaM. AMPELIN

Genus Calyptomina, RAFF.

Cal. Raflesia. Java.

Genus Chrysopteryx, Swain.

Chry. erythrorhynchus. America.

Genus Casmorhynchus, Temm.

Cas. carunculata. South America.

Genus Ampelis, Linn.

* Am. Pompadora. So. America.

Genus Rupicola, Briss.

* Rup. Peruviana. America.

Subfam. PIPRINÆ.

Genus Phœnicircus, Swain.

Phæ. carnifex. S. America.

Genus Pipra, (2) LinN.

* Pip. strigilata. S. America.

Pip. (Metopia, Swa nn.) galeata. America,

(1) Another Australian group, is considered as a subgenus of Pachy = cephala.

(2) Two subgenera have been recognized, viz.-Pipra and Metopia, Swain. 
Genus Calyptura, Swarn。

Cal. cristata. S. America and Brazil.

Genus Pardalotus, Vieili.

* Par. striatus. Australia.

Fam. MUSCICAPID Æ.

Subfam. QUERULINÆ。

Genus Querula, VIEILI.

* Qu. rubricollis. Brazil and S. America.

Genus Lathria, Swain.

Lath. cinerea. Brazil.

Subfam. PSARIAN

Genus Psaris, Cuv.

Psa. cayanensis. S. America.

Genus Pachyrhynchus, SpIx.

Pach. Cuvieri. S. America.

Subfam. FLUVICOLIN E.

Genus Gubernetes, Vigors.

* Guber. Suchii, S. America.

Genus Alecturus, VieiLl.

Al. psalurus. S. America.

Genus Fluvicola, (1) SwaIn.

Flu. cursoria. America.

Genus Seisura, VIG. and HorsF.

* Sei. volitans. Australia.

Genus Perspicilla, Swain.

P. leucoptera. S. America.

Subfant. mUSCICAPIN

Genus Rhipidura, VIG. and Horsf.

Rhi. flabellifera. Australia.

(1) Under this genus Mr. Swainson places, as a subgenus, Rle. chropus, but his type is unknown to us. 


\section{5}

Genus Monacha, VIG. and Horse.

Mo, carinata. Australia,

Genus Megalophus, Swain.

Meg. regius. S. America.

Genus Todus. (1)

To. (Conopophaga, Viejl c.) ruficeps. America.

To. (Platyrhynchus, Desm.) olivaceus. America.

To. (Todus, Linn.) viridis. S. America.

To. (Lepturus, SwaIn.) fulviceps. Brazil.

To. (Platystera, JARD. and Selby,) lobata. Africa.

Genus Muscicapa, (2) LrnN.

Mus. (Cryptolopha, SwaIn.) auricapilla. India.

Mus. (Muscipeta, Cuv.) Paradisea. India.

Mus. (Myïagra, VIG. and Horsf.) plumbea. Australia.

* Mus. (Muscicapa, Linn.) grisola. Britain.

Mus. (Hyliota, Swain.) flavigaster. West Africa.

\section{Subfam. EURYLAIMIN瓜。}

Genus Eurylaimus, Horsf.

Eu. Horsfieldii. Java.

Genus Cymbirhynchus, Vigors.

Cym. nasutus. India.

Genus Platystomus, Swain.

Plat. Blainvillii. India, Arch.

Genus Psarisomus, SwaIn.

Psa. Dalhousii. India.

Genus Serilophus, SwaIN.

Seri. lunulatus. India.

(1) Five subgeneric groups have been enumerated and typified un. der the genus Todus of Linn, viz._Conopophaga, Vieill.; Platyrhynchus, Desm.; Todus, Linr.; Lepturus, Swain.; and Platystera, Jard. and Selby。

(2) The five following groups are now considered as subgeneric forms of the genus Muscicapa, viz._Cryptolopha, Swain.; Muscipeta, Cuv.; Mÿ̈agra, Vig. and Horsf.; Muscicapa, Lin.; and Hyliota, Swain. 


\section{TRIBE II. CONIROSTRES.}

FAM. CORVID E.

Subfar. CORVIN王。

Genus Corvus, (19) Linn.

* Cor. Corax. Britain.

* Cor. (Pica, Briss.) melanoleuca. Britain.

* Cor. (nucifraga, Briss.) Caryocatactes. Europe.

Genus Coronica, (2) Gould.

Cor. fuliginosa. Austratia.

* Cor. (Barita, Cuv.) Tibicen. Australia.

Cor. (Vanga, Buff.) destructor. Australia.

Cor. (Platylophus, Swais.) galericulatus.

Genus Phonygama, Lesson.

Pho. Lessonii.

Subfar. GARRULIN Æ.

Genus Garrulus, Ray.

* Gar. glandarius. Britain.

Genus Cyanocorax, (3) Chs. Buonap.

Cy. coronatus. America.

Cy. (Cyanurus, Swain.) Bullockii. America.

Genus Dysornithia, Swain.

Dys. Canadensis. N. America.

(1) Pica, Briss. and Nucifraga, Briss. have been considered as subgenera of Corvus; though some doubts are entertained as to the value of the latter, which, is perhaps, entitled to Generic distinction.

(2) Under this genus should be placed, as a subgenus, Barita, of Cuvicr, as well as Vanga, Buffon, and Platyloplus, Swain., though there are doubts as to the appropriate station of the last.

(3) Cyanocorax is a genus instituted by the Prince of Musignano, and has the priority of G. Cyanurus of Swain. The latter, however, may be employed as a subgeneric title for that portion or group typified by Cysmurus Bullockii. 
Subfam. GLAUCOPINE.

Genus Crypsirhina, (1) Vreill.

Cry. temia. India.

Cry. (Dendrocitta, Gould.) vagabunda. India.

Genus, Ptilostomus, Swain.

Pti. Senegalensis. W. Africa.

Genus Brachystoma, Swain.

* Bra. cinerea. Australia.

Genus Glaucopis, Forster.

Gl. cinerea. Islands of the Pacific.

Subfam. CORACIN $巴$ 。

Genus Coracina, Vieill.

Cor. scutata. S. America.

Genus Cephalopterus, Geoff. St. Hilaire.

Cep. ornatus. S. America.

Genus Gymnocephalus, Geoff.

Gym."calvus. S. America.

Subfam. FREGILINÆ.

Genus Fregilus, Briss.

* Freg. graculus. Britain.

Genus Pyrrhocorax.

* Pyr. alpinus. Europe.

FAM. STURNIDÆ.

Subfam. STURNINÆ.

Genus Sturnus, Linn.

* Stu. vulgaris. Britain.

Genus Pastor, Темm.

* Pas. roseus. Britain, \&c.

(1) Mr. Swainson includes in the genus Crypsirhina, without any subgeneric division, not only the typical species of Vieillot's genus, but also those for which Mr. Gould has instituted the genus or subgenus Dendrocitta, which is here retained. 
Genus Gracula, AUCT.

* Gr. religiosa. India.

Genus Acridotheres, Vieill.

Acr. carunculatus. India and Africa.

Genus Oxystomus, (1) Swain.

Oxy, carunculatus. Isles of the Pacific.

Subam. LAMPROTORNINAE.

Genus Ptilonorhynchus, KuHL.

* Pti. holosericeus. Australia.

Pti. (Calodera, (2) Gould ) nuchale.

Genus Lamprotornis, Temm.

* Lam. leucogaster. Africa, or Lam. nitens, Africa.

Subfam. SCAPHIDURIN 2 .

Genus Astrapia, VIeIll.

As. gularis. New Groinea.

Genus Scaphidura, Swaln.

Sc. barita. South America.

Genus Quiscalus, Vieinl.

Qu. versicolor, America.

Genus Scolecophagus, Swain。

* Sco. ferrugineus. North America,

Subfam. ICTERIN虽.

Genus Cassicus, Daudin.

* Ca. cristatus. South America.

(1) Is this the same as Gould's genus Ncomorpla, mentioned in Zool. Trans.? 'The heads of two species are figured in his Syn. of Australian Birds. Swainson considers it as the scansorial type, but it appears to bear a close resemblance to the Fregilina.

(2) 'The group named Calodera, by Gould, may be considered as a subgeneric form. The typical representative was first figured in the Zool. Illust., under the title of Plilo.nuchalis. A further revision of this genus will probably shew the propriety of instituting subgene. ric groups. 
Genus Zanthornis, Cuv.

* Zan. Baltimuri. North America.

Genus Icterus, Cuv.

* Ic. castaneus. North America.

Genus, Chrysomus, Swarn.

Chry. icterocephalus. America.

Subfam. AGELAIN 压.

Genus Dolichonyx, Swain.

* Dol. oryzivora. North America.

Genus Agelaius, VierLl.

* Ag. phœniceus. America.

Genus Leistes, Vigors.

* Lei. Suchii. South America.

Genus Molothrus, Swain.

* Mol. pecoris. America.

Genus Sturnella, Vieill.

* Stur. collaris. North America.

FAM. FRINGILLIDÆ.

SubFam. COCCOTHRAUSTINæE.

Genus Coccothraustes. (I)

Coc. (Pyrenestes, Swain.) sanguinea. Africa.

Coc. (Guiraca) cœrulea. America.

* Coc. (Coccothraustes) Europæus. Britain.

Coc. (Spermophaga, Swain.) cyanorhynchus. (2) Africa.

Coc. (Dertroides, Swain.) albirostris. Africa.

(1) Under Coccothraustes Swainson enumerates five subgeneric groups, viz.-Pyrenestes, Sw.; Guîraca, Sw., afterwards changed to Coccoborus, Coecothraustes, Spermophaga, and Dertroides.

(2) The Spermophaga cyanorhynchus of Swain. is the Sper. homatina of Jard. and Selby, Illus. of Orn., N. Series, pl. XI., which title it ought, perhaps, in justice to retain.

(3) As subgeneric groups of Ploceus, besides Ploceus proper, the following are enumerated by Swainson, viz._Vidua, Cuv.; Euplectes, $\mathrm{Sw}_{\circ}$; and Symplectes, $\mathrm{Sw}$.- the latter is the same as Eupodes chrysomus 
Genus Ploceus, Cuv.

Pl. (Vidua, Cuv.) paradisea. (3) Africa.

Pl. (Euplectes, Swarn.) capensis. Africa.

Pl. (Ploceus, Cuv.) textor. India.

Pl. (Symplectes, Swain., Eupodes, Jard. and Selb.) chrysomus. Africa.

Genus Amadina (1), Swain.

Am. (Estrelda, Swain.) lineata. South Africa.

Am. (Amadina, Swarn.) melanotis. Australia.

Am. (Spermestes, Swarn.) cucullata. Africa.

Am. (Erythura, Swain.) Australia.

Am. (Pytelia, Swain.) Phœnicoptera. Africa.

Genus Tiaris, (2) Swain.

Tia. ornatus. South America.

Genus Carduelis, Briss.

* Car. elegans. Britain.

Genus Linaria, (3) Briss.

* Lin. cannabina. Britain.

Lin. (Leucosticte, Swarn.) tephrocotis. North America.

* Lin。(Chloris), vulgaris. Britain.

of Illus. of Orn., pl. X., 2d. Series, under which designation it was arranged in the collections of Sir William Jardine and Mr. Selby, long previous to the publication of the second volume of the Nat. Hist. Series of the Cabinet Cyclop.

(1) The following are considered to be the five subgeneric forms of Amadina, viz.-Estrelda, Sw.; Amadina, Sw.; Spermestes, Sw.; Erythura, Sw.; and Pytclia, Sw.

(2) Not having seen the types of Gould's genus Geospiza, their station in the series cannot be determined, but in all probability they will enter among the Coccothraustince. The groups are thus designated. Geospiza typified by Goospira magnirostris, Camarhynchus typified by Cam. Psiltacula, Cactomis typified by Cact. scandens, and Certhidea typified by Certh. olivaeca. _-See Gould in Proc. of Zool. Society, 1837, pp. 5 and 6. 'These forms belong to the Gallipagos, in which islands alone they have been found.

(3) Linaria, proper; Leucosticte, Sw.; and Chloris are supposed to be three of the subgeneric groups of this genus. 


\section{SubFam. TANAGRIN必。}

Genus Tardivola, Swain.

Tar. sphenura. America.

Genus Tanagra, (1) LinN.

* Tan. (Pitylus, Cuv.) bicolor. America.

Tan. (Spindalis, JARD. and SeLb.) bilineatus. W. Indian Islands.

Tan- (Tanagra, Linn.) Episcopus. America.

* Tan. (Cardinalis, Buon.) virginianus. America.

Tan. (Ramphopis, Vierll.) coccineus. South America.

Genus Pyranga, (2) VIEILL:

Py. (Lamprotes, Swain) rubrigularis. America.

$\mathrm{Py}$. (Pyranga, Vieill) rubra. America.

Py. (Tachyphonus, Vieill.) cristatus. America.

Py. (Leucopygia, Swain.) ruficollis. America.

Genus Nemosia, Vieill.

Nem. ruficapilla. America.

Genus Aglaïa, (3) Swain.

* Ag. gyrola. America.

Ag. (Euphonia, Vierll.) cæruleocephala. America.

Ag. (Tanagrella, Swain.) multicolor. America.

Genus Pipilo, Vieill.

Pip. maculata. America.

Pip. (Arremon, Vieizl.) torquatus. America.

(1) Swainson's subgeneric groups of this genus are Pitylus, under which he includes the Cissopus of Cuv., Tanagra, and Ramphopis, to these may be added Spindalis of Jard. and Selby, and perhaps the cardinalis of Buonop.

(2) 'The generic term of Pyranga is retained, as it was instituted prior to Phonisoma; under it Swainson enumerates four subgeneric forms, viz.-Lamprotes, Pyranga, Tachyphonus, and Leucopygia.

(3) Aglaïa, Euphonia, and Tanagrella are three subgeneric groups of the genus Aglaïa. 


\section{SubFam. FRINGILLIN $Æ$ 。}

\section{Genus Pyrgita (1), Auct.}

* Pyr. domestica. Europe, \&. .

Pyr. (Aimophila, Swain.) rufescens. America.

Pyr. (Leucophrys, Swain.) pileatus. Africa.

Genus Fringilla, (2) Linn.

Frin. (Passerella, Swa In ) iliaca. N. America.

* Frin. colebs. Britain.

Frin. (Zonotrichia, Swann.). Pennsylvania, America.

Frin. (Ammodramus, Swarn.)

Frin. (Chondestes, Swain.) strigata. Mexico, America.

Genus Emberiza, LinN.

* Em. miliaria. Britain.

Em. (Fringillaria, Swaino) capensis. Africa.

Genus Leptonyx. (3)

Lep, melanotis. S. America.

Lep. (Melophus, SwaINo) erythropterus.

Genus Plectrophanes. Meyer.

* Plec. nivalis. Britain.

Genus Agrophilus, SwaIn.

Ag. superciliosus, West Africa.

\section{Subfam. ALAUDINE.}

\section{Genus Alauda, Linn.}

\section{*A]. arvensis. Britain.}

(I) As Pyrgita seems to be more generally adopted than Passer, it is allowed to stand here. Aimophila, Sw., and Leucophrys, Sw., are two of its subgeneric types.

(2) 'The five subgeneric forms of Fringilla are Passerella, Sw.; Fringilla, Zonotrichia, Sw.; Ammodramus, Sw.; and Chondestes, Sw.

(3) Swainson considers the Emberiza crythroptera of the Illus. of ()ru. as a subgenus of Leptonyx,_to this he has given the title of Melophus, under which it is here inserted. 
Genus Calendula, (1) Linn.

* Cal. magnirostris. South Africa.

Cal. (Myrafra, Horsf.) Javanica. Java.

* Cal. (Brachonyx, Swain.) rufus. South Africa.

Genus Agrodroma, (2) Swain.

Agro. rufescens. Europe.

Genus Macronyx, Swain.

* Mac. capensis. South Africa.

Genus Certhilauda, Swarn.

Certh. longirostris. South Africa.

Subfam. PYRRHULIN $\mathbb{E}$.

Genus Pyrrhulauda, Sмrtн.

* Pyrr. cruciger, or Pyr. melanogaster. Asia and Africa.

Genus Pyrrhula, Аuct.

* Pyrr. vulgaris. Europe and Britain.

Pyrr. (Crithagra, (3) SwaIn.) sulphurata. Africa.

Pyrr. (Spermophila, Swarn.) albogularis. America.

Genus Psittirostra, Temm.

Psitt. icterocephala. Australia.

Genus Corythus, Cuv.

* Cory. enucleator. Europe and North America..

Genus Hæmorhous, Swain.

Hæmor. purpureus. North America.

Genus Loxia, Linn.

* Lox. curvirostra. Britain.

(1) Under this genus there appear to be three intermediate groups viz.-Calendula, Myrafra, and Brachonyx.

(2) This genus approaches very close to Anthus, which it represents in the circle of the Larks.

(3) Crithagra, Sw.; Spermophila, Sw., are considered as subgeneric types of Pyrrhula. 


\section{FAM. MUSOPHAGIDÆ. (1)}

\section{Subfam. PHYTOTOMINA.}

Genus Phytotoma, Molina.

Phy. rufescens, South America.

Genus, Hyreus, Steph.

Hyr. Abyssinicus. Abyssinia.

Subfam. COLIN E.

Genus Colius, LinN.

* Col. striatus. South Africa.

Subfam. MUSOPHAGIN在。

Genus Corythaix, Illiger.

* Cor. Persa. Africa.

Genus Chizarhis, WAGL.

Chi. variegata. West Africa.

Genus Musophaga, IsA RT.

Mus. violacea. Africa.

FAM. BUCERID无. (2)

Genus Buceros, Linn.

* Buc. rhinoceros. Africa.

Tribe II. SCANSORES.

FAM. RAMPHASTID正.

Genus Ramphastos, LinN.

* Ram. dicolorus, or R. Toco. S'. America.

Genus Pteroglossus, Icliger.

* Ptero. Aracari. S. America.

Genus Aulacorhynchus, Gould.

Aul. sulcatus. S. America.

(1) Not having seen Gould's genus Paradoxomis, it is uncertain whether it enters into the family of the Musopagide, or the Fringillides.

(2) A strict investigation of the Hornbills will probably show the necessity of a further division into genera, subfamilies, \&c. 
Genus Scythrops, Lat.

* Scy. Australis. Australia.

FAM. PSITTACID怎.

SUBFAM. MACROCIRCIN在.

Genus Palæornis, Vigors.

* Palæ. Alexandri. Asia, East Indies.

Genus Macrocircus, Vieill.

Mac. Aracanga. South America.

Mac. (Arara) Carolinensis, North America.

Genus Conurus, KuHL. (Psittacara, Vig.)

Con. nobilis. South America.

Con. (Psittaca, Jard. \& Swain.) leucotis. So. America.

Genus Leptorhynchus, SwaIN.

Lep. ruficaudus. South America.

Subfam. PSITTACIN瓜.

Genus Erythrostomus, Swain.

Ery. macrorhynchus. Africa.

Genus Chrysotis, Swain.

Chry. Amazonicus. South America.

Genus Psittacus, Linn.

* Psitt. erythacus. Asia, Africa.

Genus Agapornis, Selby.

Aga. cyanopterus. America.

Genus Pionus, Wagl. (Poicephalus, Swain.)

Pio. Senegalensis. Africa.

Subfam. PLYCTOlOPHIN E.

Genus Plyctolophus, Vieill.

* Plyc. sulphureus. Australia.

Genus Calyptorhynchus, Vrg. \& HorsF.

Cal. Solandri, Australia. 
Genus Nestor, WAGL.

Nes. productus. Australia.

Genus Microglossus, Geoff. St. Hilaire.

Mic. aterrimus. India.

Genus Dasyptilus (1), WAGL。

Dasyp. Pegnetii. Australia.

\section{SubFam. LORIANE}

Genus Brotogeris, Vigors.

Bro. pyrrhopterus (2), Pacific Islands.

Genus Psittaculus, Kund.

Psitt. Galgulus. Pacific Islands.

Psitt. (Coriphilus, Wagl.) Kunlir (3). S'and. Islands.

Genus Lorius, Briss.

Lor. domicellus. East Indies and Moluccas.

Genus Trichoglossus, Vigors.

* Trich. Swainsonii. Australia.

Genus Charmosyna, Wagl. (Pyrrhodes, Swarn.

Char. Papuensis. Indian Islands.

\section{SubFam. PLATYCERCINE。}

Genus Vigorsia, Swa IN.

Vig. vasa. IMadagascar.

Genus Platycercus, (4) VIG.\& Hors.

Platy. scapularis. Australia.

Genus Nanodes, VIG. and HorsF.

Non. venustus. Australia.

(1) 'This is the genus Centrourus of Swainson, but Wagler's name has the priority.

(2) In the Nat. Hist. of Parrots of the Nat. Lib. this bird is given as an aberrant form of Trichoglossus; further analysis, however, indicates it as entitled to generic distinction.

(3) 'The beautiful Cor. Kuhlii is here considered as a subgenus of Psiltaculus, to which it is nearly allied.

(1) Any of the following species may be taken as types:- $P l$. erythroterus, I'l. P'onnantii, I'l eximius, \&c. 
Genus Nymphicus, Wagl. (Leptolophus, Swain.)

Nym. Novæ-Hollandiæ (1). Australia.

Genus Pezoporus, Illiger.

* Pezo. formosus (2). Australia.

FAM. PICID $\mathbb{E}(3)$.

Subfam. PICIANÆ.

Genus Picus, Auctornm.

Picus. principalis. America.

Pi. (Hemicircus, Swain.), concretus, India.

Pi. (Dendrobates, Swa In.), fulviscapus. S. America.

* Pi. (Apternus, Swain.), Americanus. N. America.

* Pi. (Dendrocopus, Swain.), major, Britain.

Genus Chrysoptilus (4); Swain.

Chry. (Dendromus, Swain.), brachyrhynchus. Africa.

* Chry. (Chloronerpes, Swa In.), rubiginosus. S. America.

* Chry. (Dryotomus, Swain.), pileatus. N. America. Chry. (Crysoptilus, Swars.), guttatus. S. America.

Genus Malacolophus (5), Swaix.

* Mal. (Brachylophus, Swain.), viridis. Britain.

Mal. (Hemilophus, Swaın.), pulverulentus. India.

Mal. (Malacolophus, Swain.), cinnamomeus. S. Amer.

Mal. (Meiglyptes, Swain.), poicilophus. India.

Mal. (Crhysonotus, Swarn.), tridactylus. India.

(1) This is the Leptolophus auricomus of Swainson.

(2) This is the Ground Parrot of English writers.

(3) The subfamily of the Picianæ has been most carefully and successfully analyzed by Mr. Swainson, whose views are here adopted. The five groups or subgenera of Picus, as now restricted, are considered to be Picus, Hemicircus, Dendrobates, Apternus, and Dendrocouns.

(4) Under this generic head Mr. Swainson distinguishes the four following subgeneric groups:-Dendromus, Chloronerpes, Dryotomus, and Chrysoptilus.

(5) The five subordinate groups of this genus are named by $\mathrm{Mr}$. Swainson Brachylophus, Hemilophus, Malacolophus, Meiglypies, and Chrysonotus. 
Genus Colaptes (1), Swain.

Col. (Geocolaptes, Burchell), terrestris. Africa.

Col. auratus. North America.

Genus Melanerpes (2), Swain.

Mel. (Centurus, Swa In.), Carolinensis. North America.

Mel. (Leuconerpes, Swain.), candidus.

* Mel. (Melanerpes, Swain.), erythrocephalus. Americ\%。

Mel. (Tripsurus, Swain.), flavifrons. America.

Subfam. BUCCOIN㢳.

Genus Asthenurus, Swain.

Ast. exilis. South America.

Genus Picumnús, Temm.

Picum. abnormis, Asia, India." ${ }^{\text {" }}$

Genus Bucco, Linn.

Buc. armillaris. India.

Buc. (Micropogon, Tem м.) (3), Cayanensis. S. Americas Genus Pogonias, ILt.

* Pog. sulcirostris. Africa.

\section{SubFam. YUNXIN尔。}

\section{Genus Yunx, LinN.}

* Yu. torquilla. Britain.

\section{Subfam。 OXYRHYNCHINE.}

\section{Genus Oxyrhynchus, Temm.}

* Oxy. cristatus. South America.

(1) Two subgeneric groups only have been recognized under this genus, viz., Colaptes and Geocolaptes.

(2) Four subgeneric types are given by Mr. Swairson; the fifth, he supposes, to be the Picus meropirostris of Wagler, representing the Tenuirostral type.

(3) Micropogon of 'Jemm., is considered as a subgeneric form of Bucco. 
FAM. CERTHIADÆ.

SubFam. CERTHIANZE.

Genus Certhia, Linn.

* Certh. familiaris. Britain.

Genus Oxyurus, Swain.

Oxy. ornatus. South America.

Genus Dendrocolaptes, IL L. (1)

Dendro. decumanus. South America.

Dendro. (Ziphorhynchus, Swain.), procurvis. S. Amer.

Dendro. (Dendrocops, Șwain.), platyrostris. S. Amer.

Dendro. (Sittasomus, SwaIn.) sylviellus. S. America.

Dendro. (Dendroplex, Swarn.) guttatus. S. America.

Genus Sclerurus, Swain.

Scle. albogularis. S. America.

Genus Furnarius, Vieill.

Fur. melanotis. South America.

Subfam. ANABATINÆ.

Genus Geobates, SwaIN.

Geo. brevicauda. S. America.

Genus Synallaxis, Vieilı.

Syn. garrulus. S. America.

Genus Dendroma, Swarn.

Dend. caniceps. S. America.

Genus Anabates, Tемм.

Anab. Guianensis. S. America.

Genus Zenops. Illiger.

Zen. genibarbis. S. America.

\section{SubFam. SITTIN 2 .}

Genus Sittella. Swain.

Sitt. chrysoptera. Australia.

(1) Five subgeneric groups are recognized under Dendrocolaptes, viz., Dendrocolaptes, proper ; Ziphorynchus, Dendrocops, Sittasomus, and Dendroplex. 
Genus Geositta, Swain.

Geo. anthoides. S. America.

Genus Sitta, Linn.

* Sit. Europæa. Britain.

Genus Dendrophila (1), Swain.

Dendro. frontalis. India.

Genus Climacteris, Tемм.

Clim. auricomis. Australia.

SUbFAM. TROGLODYTIN $\mathbb{E}$.

Genus Platyurus, Swain.

Platy. corniculatus. South America.

Genus Thryothurus (2), Vieill。 (Scolopacinus, Buonap.)

'Thry. rectirostris. America.

Genus Troglodytes, Cuv.

* Trog. Europæus. Britain.

Genus Lochmia, Swain.

Loch. squamulata. S. America.

Genus Tichodroma, Illiger.

* Tich. muraria. Europe.

Subfam. BUPHAGIN $\mathbb{E}$.

Genus Buphaga, Latham.

Bup. Africana. Africa.

Genus Orthonyx, Temm.

Orth. spinicaudus. Australia.

FAM。 CUCULID $\rightleftarrows$.

Subfam. CUCUlin $\mathbb{E}$.

Genus Cuculus, Linn.

* Cuc. canorus. Britain.

(1) It seems doubtful whether this form is entitled to a generic distinction; is it not a subgenus of Sitta?

(2) If Gould's genus Scytalopus should prove different, or possess characters sufficiently distinct from Swainson's Platyurus, it will, in that case, rank as a subgenus of Platyurus.

(3) 'The analysis of this family by Mr. Swainson, contained in the Magazine of zoology and Botany, is here adopted. 
Genus Oxylophus, Swain.

Oxy. Edolius. Africa.

Genus Erythrophrys, Swain.

Ery. Carolinensis. N. America.

Genus Chalcites, Lesson.

* Chal, auratus, Africa.

Genus Eudynamys, Vigors. \& Horsf.

Eud. orientalis. India

Subfam. COCCYZINÆ.

Genus Serisomus, SwaIN.

Ser. cristatus. Africa.

Genus Zanclostomus, Swain.

Zan. Javanicus. Java, \&c.

Genus Coccyzus, Vieill.

* Cocc. Cayanensis. America.

Genus Ptiloleptus, SwaIn.

Pti. cristatus, S. America.

Genus Centropus, Illiger.

* Cent. Pyrrholeucus. Africa \& India.

Subfam. CROTOPHAGIN $\mathbb{E}$.

Genus Crotophaga, LinN.

* Cro. sulcirostra. America.

Genus Dasylophus, Swain.

Dasy. superciliosus.

Genus Phœnicophæus, Vieill.

Phœni calyorhynchus. Africa.

Subfam. LEPTOSTOMIN $\mathbb{E}$ 。

Genus Saurothera. VIEILL.

Sau. velata.

Genus Anadænus, Swain.

Ana. rufescens. West Indies.

Genus Leptostoma, Swain.

Lep. longicauda. South America. 


\section{Subfam. INDICATORIN尼。}

\section{Genus Indicator, LE VAILL.}

Ind. major. South Africa.

\section{Tribe IV. TENUIROSTRES.}

\section{FAM. MELIPHAGIDÆ.}

\section{Genus Meliphaga, (I) Swain.}

* Meli. Australasiana. Australia.

Meli. (Ptilotes, SwaIn.) chrysotis. Australia.

Meli. (Zanthomiza, Swain.) Phrygia. Australia.

* Meli. (Anthochæra, VIG. \& Horsf.) carunculata. Austr. Meli. (Acanthogenys (2) Gould.) rufugularis. Austr.

Genus Gliciphila, SwaIN.

Gli. fulvifrons. Australia.

G'enus Anthomiza.

Antho. cæruleocephala. Australia.

Genus Leptoglossus, Swain. (A canthorhynchus, Gouid.

Lep. tenuirostris. Australia.

Genus Ptiloturus, Swain.

Ptilo. Cafer, S. Africa.

Genus Manorina, Vieill.

* Man. viridis (3). Australia.

Man. (Gymnophrys, Swain.) torquatus. Australia.

Man. Eidopsarus, Swarn.) bicinctus. Australia.

(1) G. Meliphaga of Swainson, or rather as restricted by later authors, contains the following groups, viz._Meliphaga as now restricted, Ptilotis, Zanthromiza, Anthochora, and, perhaps, Acanthogneys of Gould.

(2) Whether this can be separated from Ptilotis, appears doubtful.

(3) Mr. Swainson makes his Gymnophrys, which answers to Gould's Homatops, and Eidopsarus, subgeneric forms of Manorina. The whole of this interesting family requires a stricter analysis than it has yet undergone before the value of the various groups it contains can be satisfactorily determined. 


\section{3}

Genus Entomiza, Swain., (Plectorhyncha, Gould.)

* Ent. cyanotis. (1) Australia.

Genus Tropidorhynchus, Vrg. and Hors.

* Tro. corniculatus, Ausiralia.

Genus Myzomela, Vig. and Hors.

Myz. cardinalis. Australia.

Genus Arachnothera, (2) Temm.

* Arach. flavigenis. India.

FAM. CINNYRID

Genus Melithreptes. VieiLl.

Melith. pacifica. Pacific Islands.

Genus Cinnyris, Cuv.

Cinny, chalybea. India and Africa.

Genus Anthreptes, Swain.

Anth. Javanica. Java, \& c.

Genus Nectarinia, ILr.I.

Nec. cyanocephala, America.

Genus Diceum, Cuv.

Dic. sanguineum. Australia and India.

FAM. TROCHILID E.

Genus Lampornis, Swain.

* Lam. mango. Brazil.

Genus Trochilus, Auct.

* Tro. longirostris. America.

Genus Cynanthus, Swain.

* Cy. forficatus. America.

Genus Phœthornis, Swain.

* Phæ. superciliosus. South America..

Genus Campylopterus. Swain。

Cam. recurvirostris. South America.

(1) The claracters of Swainson's genus Entomiza seem the same, or nearly approaching those of Gould's genus Plectorlynncha.

(2) The true situation of this genus is doubtful. 


\section{Subfam. PROMEROPID FE.}

Genus Promerops, Briss.

Pro, erythrorhynchus. Africa.

Genus Upupa, Linv.

* Up. Epops. Britain.

Genus Epimachus, Cuv.

Epim. superbus. Africa.

Subfam. PARADISIAD虑。

Genus Ptiloris (1), Swain。

Ptil. paradiseus, Austr.

Genus Paradisea, Swain。

Para. rubra. Indian Islands.

Genus Cicinnurus, Vieild.

Cicin. regius. Indian Islands.

Genus Parotia, Vieild:

Par. sexsetacea. Indian Arch.

Genus Lophorina, VIEILL。

Lop. superba. India.

\section{Tribe FISSIROSTRES.}

FAM. MEROPIDFE.

Genus Merops, Linn.

* Mer. Apiaster. Britain and Europe.

Genus Nyctiornis, Swain。

Nyc. Athertoni. India.

(1) Although the G. Ptiloris is here placed among the Paradisiadce, its affinities to Epimachus are so strong as to render it far from improbable that a stricter analysis, and better acquaintance with these hitherto little known groups, will place it among the Promeropidse, rendering it the connecting link between Promeropido, Melliphagide, and Paradisiado.

'T'he types of the Paradisiale are given with doubt, as their rarity and the difficulty of obtaining unmutilated specimens has hither. to prevented a proper analysis of the group. 
Genus Coracias, Linv.

* Cor. Garrula. Europe.

* Cor. (Eurystomus (1), Vieill.) orientalis. India.

Genus Chloropygia, Swarn.

Chlo. Leptosomus. Madagascar.

Genus Leptosomus, VicilL.

Lep. viridis. Madagascar.

\section{FAM. HALCYONIDÆ.}

Genus Tamatia (2). Auct.

Tam. maculata. America.

* Tam. (Capito, Vieicl.) leucotis. South America.

Tam. (Lypornix, Wagler.) striata. South America.

Tam. (Monassa, Vieill.) leucops. America.

Tam. (Brachypetes, Swain.) tenebrosa. South America. Genus Halcyon (3), Swain.

* Hal. sanctus. Australia.

* Hal. (Dacelo, Leach.) giganteus. Australia.

Hal. (Lyma, Lesson.) Lessonia.

Hal. (Todiramphus, Lesson.) sacer. Pacific Islands.

* Hal. (Ceyx, Lacepede.) tridactylus. India.

Genus, Alcedo (4), Linn.

* Alc. Ispida. Britain.

* Alc. (Ispida, Swain.) gigantea. Africa.

(1) The genus Eurystomus of Vieillot, (Collaris, Cuv.), is here considered in the light of a subgenus of Coracias.

(2) Under this generic name the following subgeneric groups have been recognized. Tamatia, Cuv.; Cayito, Lypornix, Monassa, and Brachypetes.

(3) Under this generic head, we have as subgenera, Halcyon, restricted, Dacelo, Lyma, Todiramphus, and Ceyx. Lyma is inserted on the authority of Mr. Swainson, no opportunity of examining a specimen having occurred.

(4) The four following forms have been separated as types of subgeneric groups. Alcedo, as restricted, 1spida, Tanysiptera, Alcyone. 
Alc. (Tanysiptera, Vigors.) Dea. India.

Alc. (Alcyone, Swain.) Australis. Australice

Genus Lamprotila, SwaIN.

Lamp. platyrhyncha.

Genus Ga lbula Linn.

* Gal. macroura, or G. albogularis. South America.

FAM. TROGONIDE.

Genus Trogon, Linn.

* Trog. fasciatus. South America.

Trog. (Harpactes, Swain.) Gouldii. Asia.

Trog. (Apaloderma, Swarn.) Reinwardii. Africa.

Trog. (Temnurus, Swain.) albicollis. West Indies and South America.

Trog. (Calurus, Swain.) resplendens. S. America.

Genus Crypticus, (1) Swarn.

Cryp. platyrhynchus. S. America.

Genus Prionites, Illiger.

* Prion. ruficapillus. S. America.

\section{Fam. CAPRIMULGide (2).}

Genus Podargus, Cuv.

Pod. humeralis. Austratia.

Pod. (Egotheles, Vig. \& Honsf.) Australis. Austratia.

* Pod. (Nyctibius, Humboldt) grandis. Australia.

(1) It is rather doubtful whether Crypticus be entitled to generic distinction. Is it not rather a subgenus of Prionites?

(2) The numerous forms and species of the Caprimulgidæ that have been discovered within the last few years, render a natural arrangement impossible, without a strict analysis and examination of the whole. In the first part of Mr. Gould's monograph of this family (the only one yet publisherl), there are no less than five new genera or subgenera characterized, several of them, it is more than probable, will be found to be merely aberrant species of genera or subgenera, previously recognized, but without examination it is difficult, indeed inipossible, to assign them their proper station. The following arrangement, therefore, is only to be considered as an imperfect attempt. 
Pod. (Batrachostomus, Gould.) auritus. Australia.

Genus Caprimulgus, LinN.

* Capri. Europæus. Britain.

* Capri. (Psalurus, Swain.) macropterus. South America. Capri. (Chordeiles, Swa in.) Americanus. America.

Capri. (Amblypterus, Gould.) anomalus. West Indies.

Genus Nyctidromus, Gould.

Nyct. Derbyanus. South America.

Genus Scortornis, Swain.

Scor, climacterus, W. Africa.

Scor. (Macrodipteryx, Swain.) Africanus. Africa.

Scor. (Semeïophorus, Gould.) vexillarius.

Scor. (Proïthera, Swain.) diurnus. America.

Genus Lyncornis, Gould.

Lyn. macrotis. India and Indian Islands.

\section{FAM. HIRUNDINIDÆ.}

Gerus Cypselus, Arist.

* Cyp. murarius. Britain.

Genus Macropteryx, Swain. (1)

Mac. longipennis. India.

Genus Chætura, Stephens.

* Chæ. macroptera. America.

Genus Hirundo, Lins.

* Hir. rustica. Britain.

\section{Order. RASORES.}

\section{FAM. PAVONIDEE.}

Genus Pavo, Linn.

* Pav. cristatus. Britain and India.

* Pav. (Argus, Temm.) giganteus. Straits of Malacca. Pav. (Polyplectron, Temm.) bicalcaratus. India.

(1) For the present a generic station is assigned to Macropteryx and Chcetura, though it is probable they may be eventually found, as Swainson considers them, subgenera of Cypselus. 
Pav. (Meleagris, Linn.) Gallipavo. Britain \& N. Amer. Genus Phasianus.

* Phas. colchicus. Britain.

* Phas. (Nycthemerus, Swarn.) argentatus. Asia.

Phas. (Tragopan) Satyrus, Asia, Nepaul.

Genus Gallus, AntıQ.

Gall. Bankiva, India and Indian Islands.

Genus Lophophorus, Tемм.

Loph. refulgens. India, Nepaul.

Genus Numida.

Num. Meleagris. Africa.

FAM。 TETRAONID正。

Genus Cryptonyx, Темm.

Cryp. coronatus. Sunda Islands.

Genus Odontophorus, VIEILL。

Odon. rufus.

Genus Tetrao, Linn. (1)

* Tetr. Scoticus. Britain.

* Tetr. (Urogallus) major. Europe.

* Tetr. (Lyrurus, Swarn.) Tetrix. Britain.

* Tetr. (Pterocles, Temm.) arenarius. Europe \& Africa.

Tetr. (Centrocircus, Swaln.) Urophasianus. N. America.

Genus Perdix, Briss.

* Per. cinerea, Britain.

Per. (Francolinus) collaris. Europe.

* Per. (Coturnix, BrIss.) Europæus. Britain.

Per. (Ptilopachus, Swıin.) erythrorlynchus. Africa.

(1) It seems lather doubtful whether the name of Tetrao should be restricted to that group of which the common Grouse, and the Ptarmi. gan are examples, or to that to which the Cock of the Wood belongs. Mr. Swainson has committed an error in his arrangement in placing the Grouse and TVood Grouse together, considering the former to possess nuked toes, like the latter. Lyrurus seems scarcely entitled to rank as a subgenus, considering it to be only one of the five forms of Tetrao, as restricted. 
Per. (Ortyx, Steph.) capistrata. America.

Genus Hemipodius, Temm. (Ortygis, Ilc.)

Hemi. Tachydromus. Asia, \&c.

Genus Tinamus, Latr. (Crypturus, Icь.)

Tin. Tataupa. South America.

Tin. (Nothurus, Wagl.) rufescens. South America.

\section{FAM. STRUTHRONID在.}

Genus Otis, LinN.

* Otis tarda. Britain, Europe.

Genus Struthio. (1)

Stru, Camelus. Africa.

Stru. (Casuarius, RAy) galeatus. Africa.

* Stru. (Dromiceus, Vieill.) Australis. Australia.

Stru. (Rhea, Brisson) Americanus. South America.

Genus Apteryx, Shaw.

Apter. Australis. Australia, New Zealand.

Fam. COLUMBID E.

Subfam. PTILINOPIN Æ.

Genus Vinago, Cuv.

Vin. aromatica. India, Africa.

Vin. (Sphenurus, Swain.) semitorquata. Africa.

Genus Ptilinopus, Swain.

Ptilin. purpuratus. Indian Islands.

Genus Carpophaga, SELBu.

Carpo. Oceanica. Indian Islands.

* Carpo. (Lophorhynchus, Swain.) dilophus. Java, Aust.

Genus Columba, LinN.

* Col. Palumbus. Britain.

Col. (Geopelia, Sw $\mathbf{1 N}$, ) lineata. India.

* Col. (Ectopistes, SwaIn.) migratoria. North America.

(1) Struthio proper, Casuarius, Dromiccus, and Rhea, are here consisidered as subgenera of G. Struthio. 
* Col. (Macropygia, Swarn.) phasianalla. Ind. Islands. Genus Turtur, Secby.

Tur, risorius. Africa.

* Tur. (CEna, Seley) Capensis. Africa.

Tur. (Chæmepelia, Swain.) cinnamominus. America.

Tur. (Leptotila, Swain.) rufaxilla. West Indies.

* Tur. (Peristera, Swain.) chalcoptera. Australia.

Genus Geophilus, Secty.

Geo. Nicobaricus. Indian Islands.

Genus Ptilophyrus (1).

* Pti. coronatus. IRolucca Islands, \& c.

Genus Opisthocomus, Hof F.

Opis. cristatus. South America.

Genus Chionis, Forster.

Chio. vaginalis (2). Australia.

FAm. CRACID在。 (Megapodinæ, SwaIn.

Genus Menura, Shaw,

* Men. Paradisea. Australia.

Men. (Megapodius, Gainard) Freycinetii. Ind. Islands and Pacific.

\section{Genus Alectura (3).}

Alect. Lathami。 Australia.

(1) Lophyrus having been used to designate a genus of insects, Mr. Swainson has changed this tille to Ptilopliyrus.

(2) Of the real affinities of this curious bird little seems to be known, and it is only to be considered as provisionally placed in its present situation.

(3) That curious bird, the Alcctura Lathami, first described by Latham as the $N w$ wolland Vulture, afterwards figured and described in the "Illustrations of Ornithology," under the title of Alectura I athami, seems more nearly allied to some of this family than any other, and we cannot agree in placing it in the circle of the Vulturida as Mr. Swainson has done, its form and characters being more decidedly Rasorial than Vulturine. It is therefore placed near to Menura and Megapodius. 
Genus Palamedea, Lins.

Pal. cornuta. America.

Genus Dicholophus, Illiger.

Dicho. cristatus. So. America.

Genus Psophia, Linn. (1).

Pso. crepitans, S. America.

Genus Crax.

Cr. globifera. S. America.

* Cr. (Ourax, Cuv.) erythrorhyncha. So. America.

Cr. (Ortalida, Merrem) garrula. South America.

* Cr. (Penelope, Lrnn.) Cumanensis. America.

Cr. (Lophocerus, Swain.) galeata. South America.

Order. GRALLATORES.

FAM. ARDEADE (2).

Genus Grus, Pall.

Gr. cinerea. Britain, Europe.

Genus Balearica, Briss.

Balear. Pavonina. Europe.

Genus Anthropoides, Vieill.

Anth. Stanleyanus. Africa.

Genus Ardea, Lins.

* Ard. cinerea. Britain.

* Ard. (Egretta, Briss.) Garzetta. Europe.

* Ard. (Butor, Antiq.) stellaris. Britain.

* Ard. ('Tigrisoma, Swarn.) lineata. South America.

* Ard. (Nycticorax, Auct.) Europæus. Britain, Europe.

Genus Cancroma, Linn.

Can. cochlearia. South America.

(1) It is doubtful whether this is the proper station of Psophia, which appears closely allied to the Cranes.

(2) The subfamilies are not yet fully established, though it is probable that the genera enumerated will, most of them, constitute the types. 


\section{Genus Platalea, LinN。}

* PIa. leucorodia. Europe.

Genus Ciconia, Briss.

* Cic. alba. Europe.

Cic. (Mycteria, Linn.) Australis. Australia.

Genus Scopus, Briss.

* Scop. Umbretta. Africa.

FAM. TANTALID在.

Genus Anastomus, It L.

Anas, lamelligerus. America.

Genus Tantalus, LinN.

Tan, loculator. North America.

Genus Ibis, Lacepede (1).

* Ib. religiosa. Asia, Africa.

Genus Ibidirhyncha, VIG.

Ibi.Struthersii. Asia, Himalaya.

Genus Hæmatopus (2).

* Hæm. Ostralegus. Britain.

Genus Aramus, Vieill.

Ar. scolopacinus. America.

\section{FAM. RALLID E.}

Genus Parra, Linn.

Par. Jacana. Asia, Africa.

Genus Purphyrio, Briss.

* Por. smaragnotus. Europe.

Genus Fulica, Lins.

* Ful. atra. Britain.

(1) The Genus Ibis, requires subdivision.

(2) The true situation of Hæmatopus is doubtful; it appears to enter this family rather than the Ardeadx, in which Swainson places it. 
* Ful. (Gallinula, Auct.) (1) chloropus. Britain.

Genus Rallus, Linn. (2)

* Ral. aquaticus. Britain.

Genus Crex, Bechst.

* Cr. Porzana, and Cr. pratensis. Britain.

Cr. (Alecthelia, Lessen) (3) lineata.

FAM. SCOLOPACIDE.

Subfan. EURYPYGIN $\mathbb{E}$.

Genus Eurypygia, ILL.

Eury. Helias. Africa.

\section{Subfam. SCOLAPACINÆ.}

Genus Scolopax, Linn.

* Sco. (Rusticola, Vieill ) communis. Brituin.

Sco. (Rhynchæa, Cuv.) Capensis. Africa.

* Sco. (Scolopax, Auct.) Gallinago. Britain.

Sco. (Macroramphus, LeAch) griseus. Europe and America.

* Sco. (Limosa, Briss.) melanura. Britain.

\section{Subfam. PHALEROPIN $Æ$.}

Genus Phaleropus, Briss.

* Phal. platyrhynchus. Britain.

Genus Lobipes, Cuv.

* Lob. hyperborea. Britain and Arctic Regions.

Genus Tringa,Lrnn.

* Trin. canutus. Britain.

(1) Swainson considers the Water Hen, Gallinula of authors, as only an aberrent species of Fulica, it is here considered as a subgeneric group of that genus.

(2) G. Rallus enters between Fulica and Crex.

(3) Not having examined this kind, its true value and station remains to be verified, but $\mathrm{Mr}$. Swainson has placed it as a subgenus of his G. Gallinula, our G. Crex. 
Genus Strepsilas, ILL。

* Strep. interpres. Britain.

\section{Subfam. HIMANTOPIN 无。}

Genus Recurvirostra, Linn.

* Rec. Avosetta. Britain, Europe.

Genus Himantopus, Briss.

* Himan. melanopterus. Britain, Europe.

Genus Totanus, Bechst.

* Tot. fuscus. Britain, Europe.

Genus Machetes, Cuv.

* Mach.pugnax. Britain.

\section{Subfam. NUMENIANR.}

Fal. Cuvieri. Africa.

Genus Falcinellus, Cuv.

Genus Numenius, (I) Briss.

* Num. arquata. Britain.

FAM. CHARADRIADE.

Genus Squatarola, Cuv.

* Squa. melanogaster. Britain.

Genus Charadrius, Lann.

* Char. pluvialis. Britain.

* Char. (Egialitis, (2) Gould, hiaticula. Britain.

Genus Arenaria, Meyer.

* Aren. calidris (3). Britain.

(1) Perhaps the Genus Ibidirhyncha should enter among the Curlews rather than the Tantalide; at all events it constitutes a beautiful connecting link between the Tantalide and the Scolopacida, by its near affinity to Numenius, and in the structure of its feet to Humatopus.

(2) 'The group of small Plovers to which our Ring Dottrel belongs has been separated by Gould from Genus Charadrius, under the title of Nirialitis; it seems to retain only a subgeneric value, and, as such, is here considered.

(:) T'he Genus Orcopholus of Jardine and Selby seems to enter 
Genus Oreopholus, JARd. \& Secby. Illust. of Orn.

Oreo. totanirostris. South America.

Genus Vanellus, Briss.

* Van. cristatus. Britain.

Van. (Pluvianus) armatus. Australia.

Genus Edicnemus, Temm.

* Edic. crepitans. Britain.

Genus Cursorius, Latr.

Cur. isabellinus. Europe, Britain, Asia.

Cur. (Ammoptila, Swain.) charadrioides.

Genus Glareola, Linn.

Glar. torquata. Europe.

Genus Erodia SALT.

Ero. Amphilensis (1). Shores of the Red Sea.

Order. NATATORES.

FAM. ANATIDE.

Subfam. Phanicoptin.E (2).

Genus Phœnicopterus, Linn.

* Phæni. ruber. Europe, Asia.

among the Charadriada, being nearly related in the structure of the feet to Cursorius, and in shape, disposition of colour, \&c., to Chara. drius.

(1) That curious bird, the Erodia Amphilensis of Salt., Diomus Ardeola, Paykull, from the for $\mathrm{m}$ of its bill, appears to be allied to the Edicmenus, while its legs, feet, and general form approach those of the Avosets : for the present it may enter here, until its direct affinities are better ascertained, for we can scarcely think it enters into the family of the Petrels, in which it is placed by Mr. Swainson.

(2) Mr. Swainson has removed the Flamingos from the Grallatorial order in which they have usually been placed, into that of the Natatores; considering that their affinities bring them into closer connection with the Anatide than any of the wading families. This view is here adopted, though with some degree of hesitation. 
Subfam. ANSERIN尼.

Genus Cygnus, Antra.

* Cyg. ferus. Britain or Cyg. Bewickii. Britain.

Genus Anser, AntiQ.

* Ans. Segetum. Britain.

* Ans. (Bernicla, Steph.) Brenta. Britain.

Genus Dendrocygna, Swain.

Dendro. arcuata. Java.

Genus Plectropterus, Leach.

Plect. Gambensis. Africa.

* Plect. Chenalopex, S герн.) Egyptiacus. Egypt, Britair.

Genus Cereopsis, Lath.

Cerop. Australis. Australia.

Subfam. ANATIN

Genus Mareca.

* Mar. Penelope. Brituin.

Genus Dendronessa, Swarv.

Dendro. sponsa. North America.

Genus Anas (1), Linn.

* An. (Chauliodus, Swaln.) Strepera. Britain.

An. (Malacorhynchus, Swain.). Australia.

* An. (Anas, Linn.) clypeata. Britain.

* An. (Boschas, Swain.) ferus. Britain.

* An. (Dafila, Leach.) caudacuta. Britain.

Genus Tadorna, LеAсH.

* Tad. Bellonii. Britain.

Genus Cairina.

Cai. Moschata, Britain or South America.

Subfam. FUligulinæe.

Genus Somateria, Lецсн.

* Soma. mollissima. Britain.

(1) 'The subgeneric groups of Mr. Swainson are here adopted. 
Genus Oidemia, Flem.

* Oid. perspicillata. America. Or Oid. fusca. Britain. Oid. (Hydrobates, Tемм.) lobatus. Australia.

Genus Fuligula, RAy.

* Ful. Marila. Britain.

Genus Clangula, Fuem.

* Clang. vulgaris. Britain.

Clang. (Oxyura, Gound.) Australis. Australia.

Genus Harelda, RAy.

* Har. glacialis. Britain.

Subfam. MERGANINE.

Genus Anserella.

Anser. Madagascariensis. Madagascar.

Genus Mergus, Linn.

* Mer. (Mergellus, Seldy.) albellus. Britain.

* Mer. Merganser. Britain.

FAM. COLYMBID E.

Genus Podiceps. Latн.

* Pod, cristatus. Britain.

Pod. (Poliocephalus, Serby) (1) Nestor. Australia.

Genus Podoa, Ilı.

Pod. Surinamensis. America.

Genus Colymbus, LinN.

* Col. glacialis. Britain.

FAM. ALCAD瓜.

Genus Uria, Brisson.

* Ur. Troile. Britain.

Genus Alca, Linn.

* Al. impennis. Arctic Regions.

Genus Mormon, ILL.

* Mor. arctica. Britain.

(1) Mr. Swainson gives to this group a generic value, but we doubt whether it is even entitled to a subgeneric rank. 
* Mor. (Mergulus) alle. Britain.

Genus Chimerina, Escholtz.

Chim. cornuta.

Genus Phaleris, Temm.

Phal, cristatella. South Seas.

Genus Aptenodytes. Forster.

* Apten. Patagonica. South Seas.

FAM. PELECANID E.

Genus Plotus, Lins.

Plo. Americanus. America.

Genus Tachypetes, VieiLI.

Tachy. Aquilinus. Tropics.

Genus Phalacrocorax, Briss.

* Phal. Cormoranus. Britain.

Genus Pelecanus, Linn.

* Pel. onocrotalus. Europe \& Africa.

Genus Sula, Auct.

* Sul. Bassana, Britain.

FAM. LARID无.

SubFam. STERNINE.

Genus Sterna.

* Ster. Hirundo, or Sterna arctica. Britain.

Genus Thalassites, Swain.

Thal. magnirostris.

Genus Phæton, LinN.

* Phæ. phœnicurus. Tropics.

Genus Rhynchops, Linn.

Rhyn. nigra. America.

Genus Gavia, Briss.

Gav. leucoceps. America, Europe, \&c.

Genus Larus, Linn.

* Lar. marinus. Britain. 
Genus Diomedia (1), Linn.

* Diom. exulans. Cape of Good Hope, \&c.

Genus Procellaria.

* Procell. capensis. Cape of Good Hope.

Genus Haladroma, ILt:

Hal. urinatrix. Pacific.

Genus Thalassidroma.

* Thal. Leachii. Britain. Or Thal. pelagica. Britain.

Genus Pachyptila, ILL.

Pachyp. Forsteri. Pacific.

(1) Under this generic head Mr. Swainson considers the following as subgenera, viz.:-Procellaria, Diomedia, Haladroma, Thalassidroma, and Pachyptila; but as they are more likely to constitute genera under a higher rank or subfamily, they are here considered as generic types. 


\section{I S T}

oF

\section{GENERIC AND SUB-GENERIC TYPES,}

TO BE PROCURED IN

BRITAIN AND EUROPE.

Neophron percnopterus.

Aquila Chrysætos,..............Golden Eagle.

Haliæetus albicilla, ............Sea, or White-tailed Eagle.

Pandion Haliæetus, ............ Osprey.

Milvus regalis, ................Common Kite.

Pernis apivorus,............... Honey Buzzard.

Buteo vulgaris, ................Common Buzzard.

Circus cyaneus,..................Hen Harrier.

Falco peregrinus, ..............Peregrine Falcon.

Accipiter Nisus, ............. Sparrow Hawk.

Astur palumbarius, ............Goshawk.

Strix flammea, ................ Barn Owl.

Ulula Stridula, ................Ivy, or Brown Owl.

Scotophilus Tengmalmi, ......Tengmalm's Owl.

Otus vulgaris, ................Long-eared Owl.

Bubo maximus, ................ Eared Eagle Owl.

Nyctea candida,................ Snowy $\mathrm{O}_{w}$.

Nyctipetes nudipes,............Little Owl.

Surnia funaria, ............... Hawk Owl.

Lanius excubitor, ..............Great Butcher Bird.

Cinclus aquaticus,..............Common Dipper. 


\section{1}

Petrocincla saxatilis,...........Rock Thrush.

Merula nigra,.................. Black Bird.

Merula musica, .................Common Thrush.

Oriolus Galbula,.................Golden Oriole.

Saxicola Enanthe, ............Wheatear.

Erythaca rubecula, ............Redbreast.

Philomela luscinia,............... Nightingale.

Curruca hortensis, .............Greater Pettychaps.

Salicaria phragmitis,........... Sedge Warbler.

Agrobates galactotes.

Drymoica cisticola.

Melizophilus provincialis,......Dartford Warbler.

Sylvia Trochilus, ..............Willow Wren Warbler.

Regulus auricapillus,............Common Gold-crest.

Egithalus pendulinus, ..........Penduline Tit.

Parus major, ...................Great Titmouse.

Accentor modularis, ............Hedge Accentor.

Motacilla Yarrellii,...............Pied Wagtail.

Budytes flava, ................ Yellow Wagtail.

Anthus pratensis, ............... Meadow Pipit.

Bombycilla garrula, ............Bohemian Wax-wing.

Muscicapa grisola,.............. Spotted Flycatcher.

Corvus Corax, .................Raven.

Pica melanoleuca, ...............Magpie.

Nucifraga caryocatactes, ....... Nutcracker.

Garrulus glandarius, .............Jay.

Fregilus gracuius, ............... Cornish Chough.

Pyrrhocorax alpinus, ...........Alpine Chough.

Sturnus vulgaris, ...............Common Starling.

Pastor roseus, .................Rose Pastor.

Coccothraustes Europæus, ...Hawfinch.

Carduelis elegans, ..............Goldfinch.

Linaria cannabina,..............Grey Linnet.

Chloris flavigaster, ............Green Linnet.

Pyrgita domestica,...............House Sparrow. 
Fringilla ccelebs, .............Chaffinch.

Emberiza miliaria,..............Corn Bunting.

Plectrophanes nivalis, ......... Snow Longspur.

Alauda arvensis,................ Skylark.

Agrodroma rufescens.

Pyrrhula vulgaris, ..............Bullinch.

Corythus enucleator,............Pine Grosbeak.

Loxia curvirostra, ............... Crossbill.

Dendrocopus major, ...........Great Pied Woodpecker.

Brachylophus viridis,...........Green Woodpecker.

Yunx torquilla, ...............Wryneck.

Certhia familiaris, ..............Common Creeper.

Sitta Europæa, ................ Nuthatch.

Troglodytes Europæus, ......Common Wren.

Tichodroma muraria,............Wall Creeper.

Cuculus canorus, ..............Cuckoo.

Upupa Epops, ................Hoopoe.

Merops Apiaster, ...............Bee-eater.

Coracias garrula, ..............Roller.

Alcedo Ispida, ................Kingsfisher.

Caprimulgus Europæus, ......Goatsucker.

Cypselus murarius, ........... Swift.

Hirundo rustica, .............. Swallow.

Phasianus colchicus, ............Pheasant.

Gallus.

Tetrao scoticus,................Red Grous.

Urogallus major, ............... Cock of the Wood.

Leiurus tetrix, .................Black Grous.

Perdix cinerea, ................ Partridge.

Coturnix Europxus, ...........Quail.

Otis Tarda,.....................Great Bustard.

Colurnba Palumbus, ...........Ringdove.

Grus cinerea,...................Crane.

Balearica pavonina, ........... Belearic Cranc.

Anthropoïdes Stanleyarus. 


\section{3}

Ardea cinerea, ................Common Heron.

Egretta garzetta, .............. Little Egret.

Butor stellaris, ...............Bittern.

Nycticorax Europæus, ......... Night Heron.

Platalea leucorodia, ............Common Spoonbill.

Ciconia alba, ...................White Stork.

Hæmatopus Ostralegus, ...... Oyster-catcher.

Porphyrio hyacinthinus.

Fulica atra,.................... Coot.

Gallinula chloropus, ...........Water Hen.

Crex porzana, ................. Spotted Crake.

Rallus aquaticus, .............Rail.

Scolopax rusticola,..............Woodcock.

Scolopax Gallinago, ............Common Snipe.

Macroramphus griseus,.........Brown Snipe, or Long-beak.

Limosa melanura, ..............Black tailed Godwit.

Phalaropus platyrhynchus, ...Grey Phalarope.

Lobipes hyperboreus, .........Red Lobefoot.

Tringa Canutus, $\quad . . . . . . . . . .$. Knot.

Strepsilas interpres, ...........Turnstone.

Recurvirostra Avocetta, ......Avocet.

Himantopus melanopterus, ...Black-backed Stilt.

Totanus fuscus, ................ Dusky Sandpiper.

Machetes pugnax,.............Ruff.

Numenius arquata,.............Curlew.

Squaterola melanogaster,......Bastard, or Grey Plover.

Charadrius pluvialis, ...........Golden Plover.

Egialitis hiaticula, ...........Ring Dottrel.

A renaria calidris, ............. Sanderling.

Vanellus cristatus,.............. (treen Lapwing.

Edicnemus crepitans, .........Thicknee.

Cursorius isabellinus, .........Cream-coloured Courier.

Glareola torquata,..............Collared Pratincole.

Cygnus ferus,...................Wild Swan.

Cygnus Bewickii, .............. Bewick's swan. 


\section{4}

Anser ferus, ...................Bean Goose.

Bernicla Brenta, ..............Brent Rarnicle.

Mareca Penelope,..............Widgeon.

Chauliodus strepera, ............Gadwall.

Anas clypeata, ................. Shoveller.

Boschas communis, ...........Wild Duck.

Dafila caudacuta, ..............Pintail.

Tadorna Bellonii, .............. Shieldrake.

Cariama moschata, ...........Muscovy Duck.

Somateria mollissima, .........Eider Duck.

Oidemia fusca, ................Velvet Scoter.

Fuligula marila,................ Scaup Pochard.

Clangula vulgaris, ............. Common Golden-eye Garrot.

Harelda glacialis, .............. Long-tailed Hareld.

Mergulus albellus, .............. Smew.

Mergus Merganser, ............Goosander.

Podiceps cristatus, ...........Great Crested Grebe.

Colymbus glacialis, ............ Northern Diver.

Uria Troile, ..................Guillemot.

Mormon fratercula, ..........Puffin.

Mergulus Alle, ................Rotche.

Phalacrocorax Cormoranus,... Cormorant.

Pelecanus onocratulus, .........Pelican.

Sula Bassana,..................Solan.

Sterna arctica, ................. Arctic Tern.

Larus marinus, ................Great Black-backed Gull.

Thalassidroma Bullockii, ......Bullock's Petrel.

Thalassidroma pelagica, .......Storm Petrel.

Puffinus Anglorum, ...........Mank's Shearwater. 


\section{A \\ L I S T \\ OF \\ GENERIC AND SUB-GENERIC TYPES, \\ TO BE PROCURED IN \\ AMERICA AND ITS DEPENDENCIES.}

Sarcoramphus Papa, ...........King of the Vultures.

Cathartes Aura,................Turkey Buzzard.

Harpyia destructor.

Ibycter ater.

Polyborus Braziliensis, .........Brazilian Kite.

Cymindis cuculoïdes.

Nauclerus furcatus.

Gampsonyx Swainsonii.

Ietinia plumbea.

Heliaptex arcticus.

Surnia funaria.

Thamnophilus striatus, .........Striated Bush-Shrike.

Ptiliogonys cinereus.

Chrysolophus ambulans. South America.

Saurophagus sulphuratus.

Tyrannus intrepidus,...........King Bird.

Milvulus forficatus.

Tyrannula audax.

Icteria polyglotta.

Dasycephala rufescens. South America. 
Myiothera capistrata. South America.

Drymophila leucopus. South America.

Pithys albifrons.

Orpheus polyglottus, ............Mocking Bird.

Pteroptichus megapodius.

Donocobius vociferans. South America.

Dulus palmarum.

Sialia Wilsonii, Blue-bird.

Cyanotus omnicolor.

Culicivora atricapilla.

Setophaga ruticilla, ...........American Redstart.

Dumecola ruficauda.

Sylvicola pusilla.

Vermivora pini, .. ............ Pine Warbler.

Mniotilla varia.

Egithina leucoptera.

Hylophilus poicilotis.

Seïurus auricapillus, Gold-crowned Thrush.

Trichas personata.

Lessonia erythronotus.

Vireo olivaceus.

Phibalura cristatus. South America.

Procnias ventralis. South America.

Chrysopteryx erythrorhynchus. South America.

Casmorhynchus carunculatus. S. America....Bell Bird.

Ampelis pompadoura. South America.

Rupicola peruviana, ............Cock of the Rock.

Phœnicurus carnifex. South America.

Pipra strigilata.

Metopia galeata. South America.

Calyptura cristata. South America.

Querula rubricollis, South America.

Lathria cincrea. South America.

Psaris Cayannensis.

Pachyrhynchus Cuvieri. South America. 
Gubernetes Suchii. South America.

Alecturus Psalurus. South America.

Fluvicola cursoria.

Perspicilla leucuptera. South America.

Megalophus regius. South America.

Conopophaga ruficeps. South America.

Platyrhynchus olivaceus. South America.

Todus viridis. South America.

Lepturus fulviceps.

Cyanurus Bullockii.

Cyanocorax coronatus.

Dysornithia Canadensis.

Coracina scutata.

Cephalopterus ornatus.

Gymnocephalus calvus.

Scaphidura barita.

Quiscalus versicolor.

Scolecophagus ferrugineus.

Cassicus cristatus.

Zanthornis Baltimore.

Icterus castaneus.

Chrysocomus icterocephalus.

Dolichonyx oryzivora.

Agelaius phoniceus.

Leistes Suchii.

Molothrus pecoris.

Sturnella collaris.

Guiraca cærulea.

Tiaris ornatus.

Leucosticte tephrocotis.

Tardivola sphenura.

Pitylus plumbeus.

Bethylus bicolor.

Spindalis bilineatus.

Tanagra Episcopus. 


\section{8}

Ramphopis coccineus. South America.

Lamprotis rubrigularis. South Americ\%.

Pyranga rubra.

Tachyphonus cristatus.

Leucopygia ruficollis.

Nemosia ruficapilla.

Aglaia gyrola.

Euphonia cæruleocephala.

Tanagrella multicolor.

Pipilo maculata.

Arremon torquatus.

Aimophila rufescens.

Passerella Iliaca.

Zonotrichia Pennsylvanica.

Chondestes strigata.

Leptonyx melanotis.

Spermophila albogularis.

Hæmorhous purpureus.

Phytotoma rufescens.

Ramphastos dicolorus.

Pteroglossus Aracari.

Aulacorhynchus sulcatus.

Macrocircus Aracanga.

Conurus nobilis.

Psittaca leucotis.

Leptorhynchus ruficaudus.

Chrysotis Amazonicus. South America.

Agapornis cyanopterus. South America.

Picus principalis.

Dendrobates fulviscapus.

Apternus Americanus.

Chloronerpes rubiginosus.

Dryotomus pileatus.

Chrysoptilus guttatus.

Malacolophus cinnamomeus. 
Colaptes auratus.

Centurus Carolinensis.

Melanerpes erythrocephalus.

Tripsurus flavifrons.

Asthenurus exilis.

Oxyrhynchus cistatus. South America.

Oxyurus ornatus.

Dendrocolaptes decumanus.

Ziphorhynchus procurvis.

Dendroceps platyrostris.

Sittasomus sylviellus.

Dendroplex guttatus.

Sclerurus albogularis.

Furnarius melanotis.

Geobates brevicauda.

Synallaxis garrulus.

Dendroma caniceps.

Anabates.

Zenops genibarbis.

Geositta anthoides.

Platyurus corniculatus.

Thryothurus rectirostris.

Lochmia squamulata.

Erythrophrys Carolinensis.

Coccyzus Cayanensis.

Ptiloleptus cristatus.

Crotophaga sulcirostris.

Anadænus rufescens. West Indies.

Leptostoma longicauda.

Nectarinia cyanocephala.

Lampornis Mango.

Trochilus longirostris.

Cynanthus forficatus.

Phothornis superciliosus.

Campylopterus recurvirostris. 
Tamatia maculata. South America.

Capito leucotis. South America.

Lypornix striata. South America.

Monassa leucops. South America.

Brachypetes tenebrosa.

Galbula macroura.

Trogon fasciatus. South America.

Harpactes Gouldii.

Temnurus albicollis.

Calurus resplendens, or

C. pavoninus.

Crypticus platyrhynchus.

Prionites ruficapillus.

Nyctibius grandis.

Psilurus macropterus.

Chordeiles Virginianus.

Amblypterus anomalus.

Nyctidromus Derbyanus.

Proithera diurnus.

Chøtura macroptera.

Centrocircus urophasianus.

Ortyx capistrata.

Tinamus Tataupa.

Nothurus rufescens.

Rhea Americana.

Ectopistes migratoria.

Chæmepelia Talpicoti.

Leptotila rufaxilla.

Opisthocomus cristatus.

Palamadea cornuta.

Dicolophus cristatus.

Psophia crepitans.

Crax globifera.

Ourax erythrorhynchus.

Ortalida garrulus. 
Penelope Marail.

Lophocerus galeatus.

Tigrisoma lineata.

Tantalus loculator.

Aramus scolopacinus.

Dendronessa sponsa.

Podoa Surinamensis.

Plotus Americanus.

Rynchops nigra. 


\author{
$A$ \\ I I S T \\ OF

\section{GENERIC AND SUB-GENERIC TYPES,}

TO BE PROCURED IN

AFRICA.

Vultur auricularis.

Neophron peronopterus.

Gypogeranus serpentarius.

Elanus melanopterus.

Aviceda cuculö̈des. West Africa.

Telophonus leucogrammicus.

Chætoblemrna leucocephala.

Nilaus capensis.

Malaconotus atrococcineus.

Prionops plumatus.

Tephrodornis virgatus.

Melasoma edolioïdes.

Analcipus bicolor.

Campephaga atrata.

Brachypus dispar.

Andropadus vociferus.

Trichophorus olivaceus.

Phyllastrephus Capensis.

Cossypha leucops. 
Crateropus Reinwardtii.

Malacocircus striatus.

Gryllivora longicauda.

Bradypetes platyurus.

Zosterops dorsalis.

Parisoma rufiventer.

Platystera lobata.

Hyliota flavigaster.

Ptilostomus Senegalensis.

Acridotheres carunculatus.

Lamprotornis leucogaster.

Pyrenestes sanguinea.

Spermophaga cyanorhyncha.

Dertroïdes albirostris.

Vidua paradisea.

Euplectes Capensis.

Eupodes chrysomus.

Estrelda lineata.

Spermestes cucullata.

Pytelia phonicoptera.

Leucophrys pileatus.

Fringillaria Capensis.

Agrophilus superciliosus.

Calendula magnirostris.

Brachonyx rufus.

Macronyx Capensis.

Certhilauda longirostris.

Pyrrhulauda crucigera.

Crithagra sulphurata.

Colius striatus.

Corythaix Persa.

Chizærhis variegata.

Musophaga violacea.

Buceros Rhinoceros.

Erythrostomus macrorhynchus. 
Psittacus Erythacus.

Pionus Senegalensis.

Vigorsia vasa.

Dendromus brachyrhynchus.

Geocolaptes terrestris.

Pogonias sulcirostris.

Buphaga Africana.

Oxylophus edolius.

Chalcites auratus.

Serisomus cristatus.

Centropus pyrrholeucos.

Phœnicophaus calayorliynchus.

Indiicator major.

Ptiloturus cafer.

Cinnyris chalybea.

Promerops erythrorynchus.

Epimachus superbus.

Leptosomus viridis.

Ispida gigantea.

Trogon (Apaloderma) Reinwardtii.

Scortornis climacteris.

Macrodypteryx Africanus.

Numida meleagris.

Pterocles arenarius.

Ptilopachus erythrorhynchus.

Struthio Camelus.

Turtur risorius.

CEna Capensis.

Scopus Umbretta.

Ibis religiosa.

Rynchæa Capensis.

Plectropterus Gambensis.

Anscrella Madagascariensis.

T'achypetes aquilinus.

Diomedia exulans.

Procellaria Capensis. 
A

\section{I S T}

OF

\section{GENERIC AND SUB-GENERIC TYPES,}

TO BE PROCURED IN

ASIA AND ITS ISLANDS.

Icthyæëtus Horsfieldii.

Spizaëtus cristatellus.

Harpagus cærulescens.

Lepidogenys Indicus.

Haliastur Pondecerianus.

Ocypterus leucorhynchus.

Dicrurus cristatus.

Ceblepyris melanops.

Oxynotus ferrugineus.

Phœnicornis flammeus.

Erucivora orientalis.

Micropus calcocephalus.

Hypsepetes psaroïdes.

Chloropsis Malabaricus.

Iora scapularis.

Hæmatornis melanocephalus.

Brachypteryx montana.

Pitta cyanura.

Chlorisoma thalassina. 
Grallaria Rex.

Myophonus metallicus.

Pellorneum ruficeps.

Pomatorhinus trivirgatus.

Timalia pileata.

Pteroptochus megapodius.

Irena puella. Java.

Thamnobia leucoptera.

Orthotomus longirostris.

Prinia familiaris.

Enicurus speciosus. Java.

Leiothrix furcatus.

Pteruthius erythropterus.

Calyptomina Rafflesii. Java.

Cryptolopha auricapilla.

Muscipeta paradisea.

Eurylaïmus Horsfieldii. Java。

Cymbyrhynchus nasut

Platystomus Blainvillii.

Psarisomus Dalhousii.

Serilophus lunulatus.

Crypsirina temia.

Dendrocitta vagabunda.

Gracula religiosa.

Acridotheres carunculatus.

Pyrrhulauda melanogaster.

Palæornis Alexandri.

Microglossus aterrimus.

Lorius Domicellus.

Charmosyna Papuensis.

Hemicircus concretus.

Hemilophus pulverulentus.

Meiglyptes poicilophus.

Chrysonotus tridactylus.

Picumnus abnormis. 
Bucco armillaris.

Dendrophila frontalis. Java.

Endynamys orientalis.

Zanclostomus Javanicus. Java.

Arachnothera flavigenys.

Anthreptes Javanica.

Paradisea rubra. Indian Islands.

Parotia sexsetacea. Indian Islands.

Lophorina superba. Indian Islands.

Nyctiornis amictus.

Ceyx tridactyla.

Tanysiptera Dea. Indian Islands.

Harpactes Gouldii.

Lyncornis macrotis.

Macropteryx longipennis.

Polyplectron bicalcaratus.

Nycthemerus argentatus.

Tragopan Satyrus.

Gallus domesticus.

Lophophorus refulgens.

Hemipodius tachydromus.

Casuarius galeatus.

Vinago aromatica.

Ptilinopus purpuratus. Indian Islands.

Carpophaga Oceanica. Indian Islands.

Leptorhynchus dilophus.

Geopelia lineata.

Macropygia Phasianella.

Geophilus Nicobaricus.

Ptilophyrus coronatus. Molucca Islands.

Megapodius Freycenetii. Indian Islands.

Ibidirhyncha Struthersii. Himalaya.

Parra Jacana.

Cursorius Isabellinus.

Dendrocygna arcuata. Java. 


\section{I S T}

OF

\section{GENERIC AND SUB-GENERIC TYPES,}

TO BE PROCURED IN

AUstralia AND isles of the PACIFIC.

Falcunculus frontalis.

Colluricincla cinerea.

Ocypterus leucorhynchus.

Ceblepyris melanops.

Grallina melanoleuca.

Cinclosoma punctata.

Sericulus chrysocephalus.

Sphecotheres viridis.

Petroïca multicolor.

Malurus cyaneus.

Acanthiza nana.

Praticola anthoïdes.

Pachycephala gutturalis.

Eopsaltria flavicollis.

Ptilochloris lunatus.

Pardalotus striatus.

Seisura volitans.

Rhipidura flabellifera.

Monacha carinata,

Myïagra plumbea. 
Coronica fuliginosa.

Barita Tibicen.

Vanga destructor.

Brachystoma cinerea.

Glaucopis cinerea. Pacific Islands.

Oxystomus carunculatus.

Ptilonorhynchus sericeus.

Calodera nuchale.

Amadina melanotis.

Scy throps Australis.

Plyctolophus sulphureus.

Calyptorhynchus Solandri.

Nestor productus.

Dasyptilus Pequetii.

Brotogeris pyrrhopterus. Pacific Islands.

Psittaculus Galgulus. Pacific Islands.

Coriphilus Kuhlii. Pacific Islands.

Trichoglossus Swainsonii.

Platycircus scapularis.

Nanodes venustus.

Nymphicus Novæ-Hollandiæ.

Pezoporus formosus.

Sitella chrysoptera.

Climacteris auricomis.

Orthonynx spinicaudus.

Melliphaga Australasiana.

Ptilotis Chrysotis.

Zanthomyza Phrygia.

Anthochæra carunculata.

Acanthogenys rufogularis.

Gliciphila fulvifrons.

Anthomyza cæruleocephala.

Leptoglossus tenuirostris.

Manorhina viridis.

Gymnophrys torquatus. 
Eidopsarus bicinctus.

Entomyza cyanotus.

Tropidorhynchus corniculatus.

Myzomela cardinalis.

Arachnothera flavigenis.

Melithreptes pacifica.

Dicæum sanguineum.

Ptiloris paradiseus.

Dacelo gigantea.

Halcyon sanctus.

Todiramphus sacer. Pacific Islands.

Alcyone Australis.

Podargus humeralis.

Egotheles Australis.

Batrachostomus auritus.

Dromiceus Australis.

Apternyx Australis.

Menura paradisea.

Alectura Lathami.

Mycteria Australis.

Cereopsis Australis.

Malacorhynchus.

Hydrobates lobatus.

Orsyura Australis.

Poliocephalus Nestor.

Aptenodytes Patagonica.

NEWCASTLE:

JRINTEY BY T. AND J. HODGSON, UNION STRHET. 

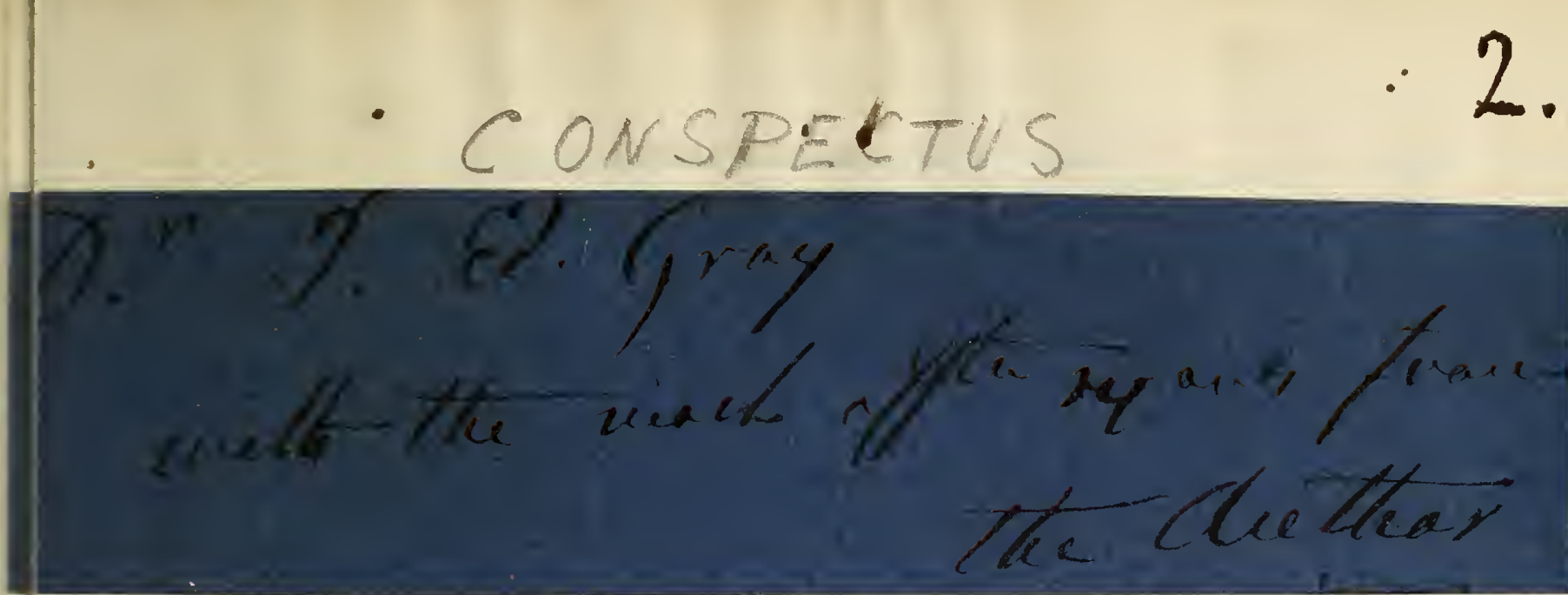

\section{SYSTEMATIS ORNITHOLOGIA.}

TISH MUSEU

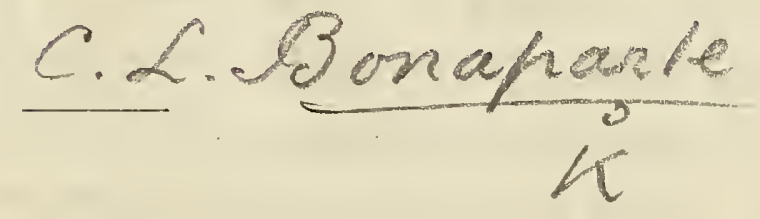

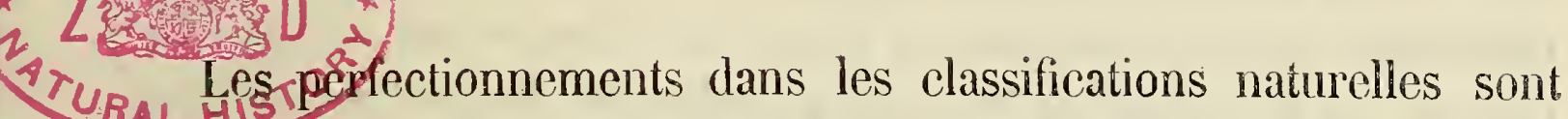
$U_{R A L}$ HIS
mecessairement des changements. Il ne faut donc pas s'étonner des variations qui s'y produisent, mais bien au contraire s'y complaire, lorsqu'elles sont basées sur de nouvelles études, sur des déductions plus justes, sur de meilleures appréciations, et surtout sur la découverte de faits nouveaux.

L'application de la doctrine des séries parallèles acquiert tous les jours de nouveaux développements, et entraine par conséquient des modifications correspondantes. Classes, ordres, familles, genres, et dans les genres la distribution elle-même des espèces, tout semble se résoudre de plus en plus en séries, à peu près comme il arrive dans un cristal qui, par le clivage, se fractionne en parties, dans lesquelles le type primitif se reproduit indéfiniment.

Bien que trop simple pour correspondre exactement à l'ordonnance réelle de la nature, le système des séries parallèles s’en rapproche cependant assez pour donner à l'esprit qui cherche les rap-ports naturels des êtres les plus utiles ressources, et mériter par conséquent tous les efforts des naturalistes par le progrès qu’il constitue à l’égard de la série linéaire.

En 1826, je pressentis la grande subdivision des Oiseaux dans les deux sous-classes que je nomme maintenant, d'après Owcn, Altrices et Pracoces, quoique le radical du premier nom se rapporte aux mocurs des parents, le second à celles de leur progénilure.

Extr. des Annales des sciences naturelles. 
Mais ce ne fut que bien des années après que, poursuivant le principe dans ses dernières conséquences, je m’affranchis, quant aux détails, du préjugé des classifications généralemént recues, en instituant, et transportant de la seconde à la première sous-classe, mes ordres des Herodiones et des Gavia, qui sont les analogues des Gralle et des Anseres, absolument comme les Couumbe le sont des Galuina. Quelques semaines se sont à peine écoulées depuis la publication de ma dernière elassification ornithologique dans les Comptes rendus de l'Académie; et je puis déjì, grièce ì de nouvelles ćtudes, ct aux nombreuses observations que j’a recues de tous côtés, apporter certaines améliorations de détail à mes séries parallèles. C'est surtout dans l'arrangement des Gallinacés, et dans la translation d'une sous-classe à l'autre des Univatones et des PTizopteri ou Manchots, qui ne sont pas plus des Palmipèdes que les Phoques ne sont des CÉTıcés, que le lecleur trouvera des changements; et c'est principalement aux remarques de M. O. des Murs, de M. Jules Verreaux, et surtout de M. Martin de Londres, que la science et moi en sommes rederables.

Bien loin de détruire mon système, les critiques bienveillantes et conlidenticlles de ce dernier, qui justifie si bien son prénom de Linnaus, n'ont servi qu'ì l'élablir sur des bases plus solides, comme M. Martin le reconnaîtra lui-même, quand il verra l'usage que j’en ai fait. Nous y puisons effectivement un nouveau parallélisme, et le plus éclatant de tous. Les Impennes du professeur Geoffroy SaintHilaire (les Ptiloptères ou Manchots), ces Phoques des oiseaux auxquels on ne saurait plus disputer te rang d'ordre à part, terminent la grande série des Altrices, eomme ses Rudipennes (les Ratites ou Autruches) leur correspondent à la fin de celle des Pracoces. 
E PI T OME.

AVES.

ALTRICES.

PRACOCES.

PSITTACI.

9. ACCIPITRES.

3. PASSERES.

1. Oscines.

2. Volucres.

\section{Zygogaclyli.}

4. INEPTI.

1. Anisodaclyli.

๖. COLUMBE.
1. Pleiodi.
2. Gyrantes.
3. Coleoramphi.

0. HERODIONES.

1. Grues.

6. Ciconiæ.

7. GAVI
1. Totipalmi.
2. Longipennes.
3. Urinatores.

8. PTILOPTERI.

9. GALLINE.

1. Passeraceic.

2. Grallacex.

1. Craces.

2. Galli.

3. Perdices.

40. GRALLE.

1. Cursores.

2. Alectorides.

11. ANSERES.

En assignant aux Struthones la place qu'ils occupent dans le tableau ci-dessus, j’ai été guidé en partie par une propriété très frappante qui semble appartenir à la classe des Oiseaux. C'est que, dans cette classe si normale, les espèces aberrantes tendent à s'élever vers les Mammifères, au contraire de ce qui advient dans ceuxci où les espèces aberrantes tendent à s'abaisser vers les Reptiles, qui eux-mêmes passent aux Poissons, comme les Poissons de leur' côté se dégradent tout à fait par leur transition si connue aux Invertébrés.

Je donne donc une nouvelle édition de ma classification, et j'en profite pour y ajouter l'énumération de tous les genres sous chaque sous-division de sous-famille ou série subordonncée. Au be- 
soin, à celle ćnumération se trouveront jointes des notes cxplicatives.

\section{Glassis 1I. AVES.}

\section{Subclassis 1. ALTRICES (Insessores).}

\section{Ordo 1. PSITTACI (Prehensores).}

i. PSITTACIDA.

a. Americamon.

1. Macrocercinz. . . . . . . . . . . Amer merid.

Genera. 1. Anodorhynchus, Spix. -- 2. Macrocercus, Vieill. (Ara, Br.). - a. Cyanopsitta, Bp. - b. Araranna, Bp. - c. Aracanga, Bp. - 3. Rhynchopsitta, Bp. - 4. Sittace, Wagl. 5. Psittacara, Spix. - 6. Cyanolyseus, Bp. - 7. Enicognathus, Gr. - 8. Nandayus, Bp. - 9. Heliopsitta, Bp.

2. Conurint. . . . . . . . . . . . America. 35 10. Conurus, Kuhl.-11. Aratinga, Spix.-12. Microsittace, Bp. - 3. Eupsittula, Bp.-14. Brotogeris, Vig. - 13. Myiopsitta, Bp. - 16. Tirica, $\mathrm{Bp}$.

3. Psittaculine. . . . . . . . . . . . Amer merid. 00 47. Psittacula, Br. - 18. Evopsitta, Bp. - 19. Pyrrhulopsis, Reich. - 20. Triclaria, Wagl. - 21. Pionopsitta, Bp. 22. Pionus, Wagl. - 23. Caica, Less. - 24. Chrysotis, Sw. 25. OEnochrus, Bp. - 26. Derotypus, Wagl.

\section{b. Delbis antiquai.}

4. Paldorntrhine, . . . . . . . . As. Ocean. 27

27. Palcornis, Vig. - 28. Belurus, Bp. - 29. Prioniturus, Wagl. -30. Tanygnathus, Wagl.-31. Psittinus, Blylh.

ij. Pezoporing. 32. Pezoporus, $\mathrm{Ill}$.

6. L'hatrenercin.

Occan. 0

33. Melopsiltacus, Could. - 34. Euphema, Wagl. - 35. Nymphicus, Wagl.-36. Psephotus, Could.-37. Barrabandius, Bp.38. Milycercus, Vig. - 39. Barnardius, Bp. - 40. Cyanoramphus, Rp. - 11. Purpureicephalus, Bp. - 12. Aprosmictus, Cimeld 
¿. Psittacine. . . . . . . . . As Afr. Oe.

a. Eelectear. - 43. Discosurus, Bp. - 44. Geeffroyus, Less. 45. Psittacodis, Wagl. - 4.6. Eclectus, Wagl.-4.7. Loriculus, Blyth.

b. Psittacese. - 48. Mascarinus, Less. - 49. Psittacus, L. 50. Poiocephalus, Sw. - 51. Agapornis, Selby. - 52. Poliopsitta, Bp. - 53. Cyclopsitta, H. et Jacq.

8. Dasuptilin.z. . . . . . . . . . . . Occan. Madag, fi Э4. Coracopsis, Wugl. - 5 . Stavorinius, Pp. - 56. Dasyptilus, Wagl.

9. Nestorine. . . . . . . . . . . . . . Occan. 3 57. Nestor, Wagl.

10. Plyctolophin.e. . . . . . . . . . . . . Ocean. 10 58. Eolophus Bp. - 59. Cacatua, Br.-60. Plyctolophus, Ill. 61. Licmetis, Ill.

2. MICROGLOSSIDE.

11. Calyptorhynchine. . . . . . . . . . . Occan. \& 62. Callocephalon, Less. - 63. Calyptorhynchus, Vig.

12. Migroglossine. . . . . . . . . . . Occan. 2 64. Microglossus, Geofr.

13. Nastrernine. . . . . . . . . . . . . . Ocean. 1 65. Nasiterna, Wagl.

\section{TRICHOGLOSSIDE.}

14. Trichoglossine. . . . . . . . . . . . Ocean. 50 66. Lathamus, Less. - 67. Trichoglossus, Vig.- 68. Chalcopsitta, Bp. - 69. Eos, Wagl. - 70. Lorius, Br. - 71. Charmosina, Wagl. — 72. Coriphilus, Wagl.

\section{STRIGOPIDA:}

15. Strigopine . . . . . . . . . . . . Ocean. I

73. Strigons, $G r$. 
Ordo 2. ACCIPITRES (Rapaces).

3. VULTURIDA.

16. Catilartina. . . . . . . . . . America.

74. Sarcoramphus, Dum. - 75. Gryphus, Geoffr.- 76. Cathartes, Ill. - 77. Coragyps, Geo/fr.

17. Vulturinde.

Eur. As. Alr. 13

78. Gyps, Sav. - 79. Vultur, L. - 80. Otogyps, Gr. - 81. Neophron, Sav.

6. GYPAETIDE.

18. Gypaetind. . .

Eur. As. Afr.

82. Gypaetus, Storr.

7. GYPOHIERACID

19. Gypolineracina.

Africa.

83. Gypohierax, Rupp.

8. FALCONIDE.

20. Aquilin. • . • . . . . . . . . Cosmopolit. 38

a. Aquileax.-84. Aquila, Br.-a. Aquila, Kp.- b. Uroetus, Kp. - c. Plercelus, Kp. - 85 . Entolmatus, Blylh. - 86. Onychæetus, Líup. - 87. Helotarsus, Smilh.

b Haliaeteac. - 88. Haliactus, Sav. - a. Halicelus, Kp. -

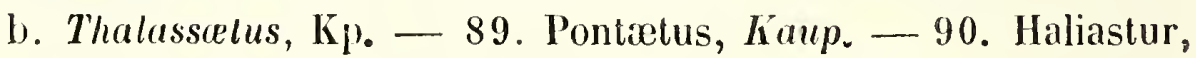
Selb. - 91. Heterachus, liaup.

c. Pandioneax. - 92. Pandion, Sav. - 33. Polioselus, haup.

1. Circarteare. - 94. Cirçelus, Vieill. - 9̈. Spilornis, Gr. 96. Herpelotheres, Vieill.

21. Butloninas. . • . • • . • . . C Cosmopolit.

a. Butconeac. - 97. Archibuteo, Brehr. - 98. Buteo, Cuv. - a. Buteo, $\mathrm{K}_{\mathrm{P}}$. - b. Peccilopternis, $\mathrm{K}_{\mathrm{p}}$. - 99. Tachytriorchis, $K p$. - 100. Buteogallus, Less.- 101. Ichthyoborus, $K$ p. - 102. Polionis, Kip. - a. Poliornis, Kp. - b. Pernopsis, 1)ubus. - 103. Kaupifalco, $B p$.

b. Asturincese-104. Asturina, Vicill.- 105. Leucopternis, Kip.

a. Milvear. - 106. Milus, br. - 107. Lophoiclinia, Kr. 10.8. ligpoictinat lip. 
b. Perneae. - 109. Pernis, Cuv. - 110. Avicida, Sw. 111. Lophastur, Blyth. - 112. Nacheiramphus, Schleg. 113. Rosthramus, Less. - 114. Odontriorchis, Kp. - 115. Regerhinus, $K p$.

c. Elaneae. - 116. Nauclerus, $A g$. - 117. Chelidopteryx, Ip . - 118. Elanus, Sav. - 119. Gampsonyx, Vig. - 120. Ictinia, Vieill. - a. Ictinia. Kp. - b. Pocilopleryx. Kp.

23. Falconin.e. . . . . . . . . . . Cosmopolit.

a. Falconcae. - 121. Jeracidea, Gould. - 122. Falco, $L_{\text {. }}$ 123. Hierofalco, Cuv. - 124. Gennaja, $h^{\prime} p$. - 125. Chiquera, Bp. - 126. Hypotriorchis, Boie.-127. Esalon, Kp.

b. Harpageae. - 128. Harpagus, Vig. - 129. Spiziopteryx, K $p$.

c. Tinnunculeae. - 130. Tinnunculus, Vieill.-a. Timmunculus, Kp. - b. Tichornis, Kp. - c. Pecilornis, Kp. - 131. Erythropus, Brehm. - 132. Polioierax, Kaup. - 133. Jerax, Vig.

24. Accipitrinz. . . . . . . . . . . . Cosmopolit.

a. Spizateae. - 134. Lophætus, Kp. - 135. Pternura, Kp. 136. Spizætus, Vieill. - 137. Jerætus, Kp. - 138. Limnætus, Vig. - 139. Spizastur, Less.

b. Morphneae. -140 . Thrasætus, Gr. - 141. Harpyhaliætus, Lafr. - 142. Morphnus, Cuv. - 143. Hypomorphnus, Cab. -144. Urubitinga, Less. - 145. Craxirex, Gould. - 146. Dædalion, $B p$.

c. Accipitreac.-147. Rhynchomegus, $B p .-148$. Micrastur, Gr. - 149. Lophospizia, Kp. - 150. Leucospizia, Kp.-151. Astur, Bechst. - 152. Cooperastur, Bp.

133. Geranospiza, Kp. -154 . Melierax, Gr. -155 . Sparvius, Vieill. - a. Sparvius, Bp. - b. Tuchyspizia, Kp. - c. Scelospizia, Kp.-156. Nisus, Bp.-157. Urospizia, Kp.-158. Micronisus, Gr. - 159. Accipiter, Br. - 160. Jeraspizia, $K p$.

25. Circine.

161. Circus, Lacép. - 162. Spiziacircus, Kp. - 163. Strigiceps, Bp. - a. Spilocircus, Kp. - b. Plerocircus, Kp.—c. Strigiceps, $\boldsymbol{K} \mathbf{p}$.

26. Polyborink.

Amer. m. Madag.

a. Ibictereae -164 . Etotriorchis, $K p .-165$. Daptrius, Vieill. -166. Ibicter, Vieill. - 167. Milvago, Spix. - 168. Phalcobænus, Lafr.

b. Polyboreae. - 169. Polyborus, Vieill.

c. Polyboroideare - 170. Polyboroides, Smilh. 
9. (TPOCERANIDE.

27. Givrogeranina.

Africa.

171. Gypogeranus, 111 .

10. STRIGID E.

28. Strigine. • • • • . . . . . . . . Cosmopolit. 19

172. Strix, L. - a. Megastrix, Kp. - b. Dactylostrix, Kp. -

c. Strix, Kp. - d. Sceiostrix, Ki . - 173. Phodilus, Geoffr.

29. Ulubine. . • . . . . - . . . Cosmopolit.

a. Uludege. - 174. Ulula, Cuv. - 175. Plynx, Blyth.176. Nyctale, Brehm.

b. Syrnieae. - 177. Syrnium, Sav. - 178. Macabra, Bp.179. Myrtha, Bp. - 180. Bulaca, Hodgs.-181. Cicaba, Wagl. - 182. Pulsatrix, Iíp. - 183. Gisella, BPp.

c. Dtcre. - 184. Otus, Cuv. - a. Otus, Kp. - b. Rhinoptinx, Kip. - 185. Nyctalops, Wagi.-186. Brachyotus, Boie.187. Phasmatoptynx, $K p$.

d. Bubonexe. - 188. Bubo, Cuv. - 189. Nisuella, Bp.190. Nyctætus, Geoffr. - 191. Urrua, Hodgs. - 192. Megaptynx, Bp.-193. Ascalaphia, Geo/fr.-194. Pseudoptynx, Iíp.

30. Surning.

Cosmopolit.

a. Seopere. - 195 . Ketupa, Less. - 196. Lophostrix, Less. 197. Ephialtes, Bp. - 198. Acnemis, Kaup. - 199. Asio, Schleg.-200. Lempijius, Bp.—201. Scops, Sav.—202. Pisorhina, Iip.

b. Atheneze. - 03. Scotopelia, Bp. - 204. Ctenoglaux, hip.205. Pholeoptynx, $K p .-206$. Athene, Boie._ 207. Gymnasio, $B p$.

c. Heroglauceae. - 208. Ieroglaux, Kp. - 209. Rabdoglaux, Bp. - 210. Spiloglaux, Kp. - 211. Sceloglaux, Kp. 212. Tænioglaux, Kp.—213. Tænioptynx, Kp.—214. Phalænopsis, Bp. - $21 \ddot{3}$. Microglaux, Kp.

d. Sumicae. - 216. Nyctea, Stcph. - 21\%. Surnia, Dumér. 218. Glaucidium, Boie. 
Ordo 3. PASSERES.

\section{Tribus 1. DCHNWS.}

\section{Stirps 1. CULTRIROSTRES.}

1. CORVIDE.

31. Corvine.

Cosmopoi. 40

a. Corveae. - 219. Corvultur, Less. - 220. Corvus, L. a Corvus, Kp. - b. Corone, Kp. - c. Trypanocorax, Bp. d. Amblycorax, Bp. - 221. Lycocorax, Bp. - 222. Physocorax, $B p$. 223. Gazzola, Bp. - 224. Monedula, Brehm.

b. Gymmocorveae. - 225. Gymnocorvus, Less. - 226. Picathartes, Less.

32. Nugifragin.x. Eur. As. Am. s.

227. Nucifraga, Br.-228. Picicorvus, Bp. -229. Podoces, Fischer.

33. Baritine. Oceania. 16 230. Strepera, Less. - 231. Barita, Cuv. - 232. Cracticus, Vieill. - 233. Bulestes, Caban. - 234. Pityriasis, Less.

34. Fregiline. Eur. As $\mathbf{s}$

235. Pyrrhocorax, Vieill. - 236. Fregilus, Cuv.

\section{GARRULID $\approx$.}

3ə̈. Garrilinz.

Cosmopol.

a. Picaceac.-237. Pica, Br.-238. Streptocitta, Bp.-239. Cyanopica, Bp. - 240. Psilorhinus, Rupp. - 241. Biophorus, Schlegel. - 242. Calocitta, Bp. - 243. Cyanurus, $B p$.

b. Garruleae. - 244. Xanthoura, Bp. - 245. Uroleuca, Bp. 246. Cyanocorax, Boie. - 247. Cissilopha, Bp. - 248. Cyanocitta, Strickl. - 249. Garrulina, Bp. - 250. Aphelocoma, Cab. - 251. Gymnokitta, Wied. - 252. Cyanogarrulus, Bp. 253. Garrulus, $B r .-254$. Perisoreus, $B p$.

36. Ptylorhynchine. . . . . . . As. m. Ocean.

255. Kitta, Kuhl.-256. Ptilorhynchus, Kuhl-257. Alluroedus, Cab. - 258. Chlamydera, Gould.

37. Myiophonina. As. m. Ocean.

259. Myiophonus, Temm. - 260. Arrenga, Less.

Extr des Annales des sciences naturelles. 
38. Crupsinilinin.e. As. Afr. Oc. 13

261. Crypsirhina, Vieill. - 262. Glenargus, Cab. - 263. Temnurus, Less. - 264. Dendrocitta, Gould. - 265. Ptilostomus, Sw. - 266. Struthidea, Gould.

\section{STURNIDE.}

39. Lamprotornithine.

As. Afr. Oc. 58

a. Dny chognatheae. - 267. Sturnoides, $H$. et Jacq.-268. Lamprocorax, Bp.-269. Onychognathus, Harll-270. Amydrus, $C a b$. 271. Pilorhinus, $C a b$. - 272. Naburupus, Bp. 973. Cinnamopterus, $B p$.

b. Lamprotomitheae. - 274 . Spreo, Less. - 275. Notauges, Cab. - 276. Calornis, Bp. - 277. Lamprocolius, Sundev.278. Urauges, Cab. - 279. Juida, Less. - 280. Lamprotornis, Temm. - 281. Enodes, Temm. - 282. Aplonis, Gould.283. Saraglossa, Hodgs. - 284. Hartlaubius, Bp.

40. Stunnine. . . . . . . . Eur. As. Afr. Oc. 40

283. Sturnus, L. - 286. Pastor, Temm. - 287. Gracupica, Less. - 288. Sturnopastor, Hodgs. - 289. Heterornis, Gr. 290. Sturnia, Less. - 291. Temenuchus, Cab. - 292. Acridotheres, Vieill. - 293. Dilophus, rieill.

41. Graculine.

As. Ocean. 8

294. Gymnops, Cuv. - 295. Mino, Less. - 296. Gracula, L.297. Ampeliceps, Blyth. - 298. Basilornis, Temm. - 299. Melanopyrrhus, Bp.

4. 2. Buphagine.

Afr. Ocean. 3

300. Scissirostrum, Lafresn. - 301. Buphaga, L.

\section{ICTERIDE.}

43. Quiscalina.

America.

302. Scolecophagus, Sw. - 303. Quiscalus, Vieill. - 304. Scaphidurus, $S w .-305$. Hypopyrrhus, Bp. - 306: Lampropsar, Cab.-307. Aphobus, Cab.-308. Molothrus, Sw.-309. Cyrtotes, Reich.

44. Icternin.

America. 112

a. Cassiceas - 310. Clypiclerus, Bp.-311. Ocyalus, Waterh. - 312. Ostinops, Cab. - 313. Cassicus, Ill. - 314. Cassiculus, $S w .-315$. Archiplanus, $C a b$.

b. Ictereas - 316. Icterus, Br. - 317. Xanthornus, Cuv. 318. Gymnomystax, Reich. - 319. Xanthosomus, Cab.320. Hyphantes, Vieill. - 321. Jendulinus, Vieill. 
c. Agelreieac. - 322. Trupialis, Bp. - 323. Pedotribes, $C a b$. - 324. Amblycercus, Cab.- 325. Amblyramphus, Leach. 326. Leistes, Vig. - 327. Xanthocephalus, Bp. - 328. Agelæius, Vieill. - 329. Thilius, Rp.-330. Dolichonyx, Sw.

Stirps 2. CONIROSTRES.

15. PLOCEIDE.

45. Ploceinæ.

Africa As. Oc. 70

331. Alecto, Less. - 332. Sycobius, Vieill. - 333. Sycobrotus, Caban.-334. Nelicurvius, Bp. - 335. Hyphanturgus, Cab. - 336. Hyphantornis, Gr. - 337. Textor, Bp.-338. Ploceus, Cuv. - 3.39. Sitagra, Reich. - 340. Quelea, Reich. 341. Foudia, Reich.-342. Ploceipasser, Smilh. - 343. Nigrita, Strickl.

46. Viduina.

Africa.

344. Pyromelana, Bp.-345. Euplectes, Sw.-346. Coliipasser, Rupp. - 347. Urubrachya, Bp. - 348. Chera, Gr. - 349. Steganura, Reich. - 350. Vidua. Cuv. - a. Tetroenura, Reich. b. Videstrelda, Lafr. - 351 . Hypochera, $B p$.

47. Estreldine.

As. Afr. Ocean.

a. Pyrenesteae. - 352. Spermospiza, Gr.-3ə3. Pyrenestes, Sw. - 354. Coryphegnathus, Reich. - 355. Sporopipes, Cab.

a Amadineae. - - 356. Padda, Reich. - 357. Munia, Hodgs. - 358. Uroloncha, Cab. - 3599. Spermestes, Sw.-360. Amadina, Sw.-361. Donacola, Gould.-362. Sporothlastes, Reich. - 363. Stagonopleura, Reich. - 364. Pœphila, Gould. 365. Erythrura, $S w .-366$. Neochmia, H. et Jacq.-367. Otygospiza, Sundev.-368. Pytelia, Sm.- 369. Emblema, Gould. - 370. Habropyga, Cab.

c. Estreldeac. - 371. Lagonosticta, Cab. - 372. Uræginthus, Cab.-373. Zonæginthus, Cab. - 374. Egintha, Cab.375. Sporæginthus, Cab. - 376. Estrelda, Sw. - a. Estrelda, Bp., 1850 . - b. Neisna, Bp., 1850.

\section{FRINGILLIDE.}

48. Passerina.

Eur. As. Afr. 30

377. Philaterus, Sw. -378 . Passer, Br. -379 . Pyrgita, Cuv.

-380 . Pyrgitopsis, $B$ p. -381 : Corospiza, $B$ p. 
49. Frimghimas. Eur. As. Afr. Am. 100

a. Fringllleae. - 382. Mycerobas, Cab. - 383. Hesperiphona, Bp. - 384. Eophonia, Gould. - 385. Coccothraustes, Br. 386. Callacanthis, Reich. -397 . Fringilla, L. -388 . Petronia, Kp. - 389. Gymuoris. Hodgs. - - 390. Xanthodina, Sund.

b. Cardueleae. - 391. Hypoxanthus, $C a b$. -392 Chrysomitris, Boie. - 393. Astragalinus, Cab. - 394. Pyrrhomitris, Bp.395. Carduelis, $\mathrm{Br}$.

c. Serineze. - 396. Crithagra, Sw. - 397. Poliospiza, Schiff-398. Citrinella, Bp. -399 . Serinus, Koch. -400 . Metoponia, $B p$.

d. Pyrrhuleac. - 4.01. Pyrrhula, Br. - 402. Pyrrhoplectes, Hodgs.

:0. LOXIINE.

Eur. As."Am. s. 46

a. Loxieas - -403 . Chaunoproctus, $B p .-404$. Hematospiza, $B l y l h$. - 405. Loxia, Br. - 406. Corythus. Cuv. - 407. Spermopipes; Cab. - 408. Uragus, Keys. et Bl.

b. Carpodaceae. - 409. Pyrrha, Cab. - 4.10. Pyrrhospiza, Hodgs. - 411 . Propasser. Ilodgs. - 412 Carpodacus, Bp. 43. Pyrrhulinota, Hodgs. - 414. Erythrospiza, $k p-a$. Rhodopeclyys, Cab. - b. Buchanetes, Cab.

c. Montifringilleae. - 415 . Leucosticte, Sw. - 416. Montifringilla, Brehm. - 417. Fringalauda, Hodgs.

d. Limotere. - 418. Linota, Bp. - 419. Acanthis, leys.

31. Psittinostrink. . . . . . . . . Ocean. 2 200. Psittirostra, Temm. - 421 . Hypoloxia, Licht.

52. Geospizine.

Ins. Gallopag. 1:: 422. Geospiza, Ciould. - 423. Camarhynchus, Gould. - \$24. Piezorhina, Lafr. - 425. Cactornis, Gould. - 426. Certhidia, Giould.

83. Limenazind. . . . . . . . Eur. As. Afr. Am. s 40 427. Cynchramus, B1. - 428. Plectrophanes, Mey. - 429. Centrophanes, Kp. - \$30. Onychospina, Bp.—431. Emberiza, $L$. - 432. Buscarla, Bp. - 433. Schenicola, Bp.-434. Horlulants, lip.- $43 \%$. Fringillaria, Sw. - 435. Hypocentor, Cab.

a Ronotrichie: - 437. Granativora, Bp. - 438 . Oritura, Bp. - 139. Hirmophili, Sw. - \$10. Chondestes, Sw. - 44. Zomolrichia, Sw. - \$2. Chrysongega, Bp. - \$43. Euspiza, Bp. 
CONSPECTUS SYSTEMATIS ORNITHOLOGIE.

- 44 . Spizella, Bp. -45 . Passerculus, $B p .-446$. Peucia, Aud. - 447. Coturniculus, Bp.-448. Ammodromus, Sw. 449. Emberizoides, Temm. - 4300. Sycalis, Boie. - 451. Mclanodera, $B p .-452$ Gubernatrix, Les\$s.

b. Struthese - 453. Calamospiza, Bp.-4.̈4. Diuca, Reich.455. Phrygilus, Cab. - 456. Rhesdespina, Cab.-457. Passerella, Sw. - 458. Struthus, Bp.-459. Junco, Wagl. 460. Poospiza, Cab. - 461. Cocopsis, Reich. - 469. Paroaria, Bp. - 463. Lophospiza, Bp. - 464. Tiaris, Sw. - 465. Melophus, $S w$.

c. Spizeac. -466. Spiza, Bp.-467. Hoplospiza, Cab.-468. Volatinia, Reich.

d. Pipiloneare. - 469. Pipilo, Vieill.-470. Pyrgisoma, Pucheran. - 471. Arremon, Vieill. - 42. Phænicophilus, Strickl. - 473. Buarremon, Bp. - 474. Embernagra, Less.-47ä. Donacospiza, $C a b$. - 476. Pipilopsis, Bp.— 477. Thlypopsis, $C a b$. -478. Allapetes, Wagl.-479. Chlorospingus, Cab.-480. Hemispingus, Cab. - 481. Pyrrhocoma, Cab. - 482. Cypsnagra, Less.

5:. Pityline.

America. 100

a. Pityleac. - 483. Coccoborus, Cab. - 484. Caryothraustes, Reich - 485. Periporphyrus, Reich. - 486 . Pitylus, Cuv. a. Pilylus, Reich.- b. Cissurus, Reich.- 487. Cyanoloxia, Bp. -488. Guiraca, Sw. - 489. Cardinalis, Bp. - 490. Pyrrhuloxia. $B p$.

b. Spermophileac - 491. Oryzoborus, Cab. - 492. Melopyrrha, Bp. - 493. Pyrrhulagra, Schiff. - 494. Catamblyrhynchus, Less. - 495 . Catamenia, Bp. - 496 . Phonipara, Bp.497. Spermophila, Sic. - a. Leucomeluner, Bp. - b. Pyrrhomelance, Bp. - 493. Sporophila, Cab. - 499. Callirhynchus, Less.

c. Saltatorea - -500 . Psittospiza, Bp. - 501. Lamprospiza, Bp. - 50 2. Diucopsis, Bp.-503. Orchesticus, Cab. -50 '. Bethylus, Cuv. - 505. Saltator, Vieill.

\section{Stirps 3. SUBULIROSTRES.}

\section{TURDIDE.}

56. Tundine. .

Cosniopol. 100

506. Zoothera, Vig. - 507. Myiophaga, Less, - 308 . Oreocincla; Gould. - 509. Cichlherminia, Rp. -510 . Turdus, L. - 
511. Planesticus, Bp. - 512. Cichloselys, Bp. -513 . Myiocichla, Schiff. - 514. Merula, Ray. - 515. Geocichla, Kuhl. 516 . Dulus, Vieill. - 517 . Catharus, Bp.

57. Saxicolinx.

Cosmopol. 150

a. Monticoleac. - 518. Monticola, Boic. - 519. Petrocossyphus, Boie. - 520. Orocetes, Gr. - 521. Grandala, IIodgs. 522. Kittacincla, Gould. - 523. Copsychus, Wagl.- 524. Gervaisia, Bp. - 525. Pooptera, Bp. - 526. Bessonornis, Smith. - 527. Thamnolæa, Cab. - 528. Dromolæa, Cab.

b. Saxicoleave - 529. Parisoma, Sw. - 530. Bradornis, Smith. - 531. Sigelus, Caban.-532. Agricola, Verr.-533. Myrmecocichla, Cab. - 534. Campicola, Sw.- 535 . Saxicola, Bechst. - 536. Pratincola, Koch. - 537. Oreicola, Bp.

c. Lusciniere. - 538 . Hodgsonius, Bp. - 539. Ajax, Less. 540 . Myiomela, IIodgs. - 541. Pogonocichla, Cab.-542 Sialia, Sw. - 543. Niltava, Modgs. - 544. Petroica, Sw. 545. Erythrodryas, Gould. - 546 . Drymodes, Gould. 547. Miro, Less. - 3̈48. Janthia, Blyth. - 549. Larvivora, Hodgs. - 550 . Adelura, Bp. — 591 . Chæmorrhous, Hodgs. 552. Ruticilla, Ray. - 553. Cyanecula, Br. - 554. Rubecula, $B r$. - 553. Calliope, Gould. - 556 . Philomela, Br.

19. Srluinde.

Eur. As. Afr. 50

a. Sylvieae. - 557. Adophoneus, Kaup. - 538. Curruca, Br. 559. Sylvia, /3p.— $\$ 60$. Sterparola, Bp.-561. Pyrophthalma. lip. - 562. Melizophilus, Leach.

b. Phyllopscusteac. - 563. Phyllopseuste, Meyer.-564. Abrornis, Hodys.— 65 . Horornis, Hodgs.— 566 . Geobasileus, Caban.

69. Cialanoherpixa.

Eur. As. Afr. 190

a. Sphennere. - 567 . Cynchlorhamphus, Gould.- 568 . Heterurus, Hodys. - 569. Eurycercus, Blyth. - 570. Megalurus, IIorsf. - 571. Sphenacacus, Strickl. - 572. Poodytes, Cab. \$73. Sphenura, Licht. - 574. Chactornis, Sw.

b. Canamoherpearo. - 575 . Tatare, Less. - 576. Bernieria, Bp. - 577. Myyllastrophus, Sw. - 578. Calamoherpe, Meyer. 579. Calamodyta, Meyer. - 580. Lusciniola, Gr.— - 81 . Lusciniopsis, Bp. - 582. Bradypterus, Sw. - 583 . Cettia, Bp. 584. Tribura, IIodys. - 585. Neornis, IIodgs. - \$86. Chloropeta, Sinith. - \$87. Hypolais, Brehm. - \$88. Iduna, Keys.

r. Iocenstellear. - \$89. Locustclla, Gould. - \$90. Calamanthus,

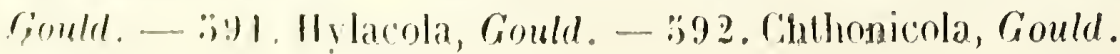


d. Edoneae. - 593. Chætops, Sw.-594. Cercotrichas, Boie.595 . Pentholæa, Cab. - 596. Thamnobia, Sw. - 597. Edon, Boie.

e. Drimoiceac. - 598. Orthotomus, Horsf. - 599. Arundinax, Blyth. - 600. Horictes, Hodgs. - 601. Daseocharis, Cab. 602. Prinia, Horsf.-603. Dumetia, Blyth.-604. Suya. Hodgs. - 605. Cisticola, Less. - 606. Catriscus, Cab. - 607. Apalis, Sw.-608. Drymoica, Sw.-609. Drymoipus, Bp.-610. Hemipteryx, Sw.-611. Tesia, Hodgs. - 612. Pnoepyga, Hodgs. - 613. Comaroptera, Sundev. - 614. Sylvietta, Latr. 615. Syncopta, $C a b$.

60. Accentorine.

Eur. As. Afr. Oc.

a. Accentoreas. - 616 . Cinclosoma, Gould. - 617. Accentor, Bechst. - 618. Prunella, Vieill. - 619. Origma, Gould.

b. Acanthizeze. - 620. Sericornis, Gould. - 621. Gerigone, Gould. - 622. Pyrrholæmus, Gould.-623. Acanthiza, Vig. 624. Picnosphrys, Strickl. - 625. Culicipeta, Blyth.

18. MIALURIDE.

61. MaLuRin $\pi$. Ocean. 14

626. Malurus, Vieill. - 627. Stipiturus, Less - 628. Amytis, Less.

\section{TIMALIID 2 .}

62. Garrulagina.

Asia, Ocean. 40

629. Lophocitta, Gr. - 630. Garrulax, Less. - 631. Jantho. cincla, Gould. - 632. Leucodioptron, Schiff. - 633. Trochalopteron, Hodgs. - 634. Pterocyclus, Gr. - 635. Actinodura, Gould. - 636. Otagon, Mus. Lugd. - 63\%. Keropia, Gr. 638. Cutia, Hodgs. - 639. Alcopus, Hodgs. - 640. Malacias, Cab.

63. Psophodina.

As. m. Ocean. 10

641. Psophodes, Horsf. - 642. Oreoica, Gould. - 643. Sphenostoma, Gould.-644. Xerophila, Gould.-645. Melanochlora, Less.

64. Crateropodine. As. Afr. Oc. 40

646. Phyllanthus, Less. - 647. Crateropus, $S w$. - 648. Argya, Less. - 649. Malacocercus, $S w .-650$. Gampsorhynchus, Blylh. -651. Pomatostomus, Cab.-652. Pomatorhinus, Horsf. -653. Xiphoramphus, Blyth. 
6:. MIMIN

America .

6:34. Mimus, Buie. - 655. Orpheus, $5 w$. - 656. Melanotis, Bp. - 637. Galeoscoptes, $C a b$. - 6.38. Felivox, Bp. - 659. Donacobius, Sw.-660. Buglodytes, Bp.-661. Harporhynchus, Bp.

66. Brachutodin.s.

a. Brachipodinge. -66 2. Picnonotus, $h u h i .-663$. Ixos. Temm. - 66 '. Brachypus, Sw._663. Otocampsa, Cab._-666. Lodorusa, $C a b .-667$. Apalopteron, Schiff. - 668. Trachycomus, Cab. - 669. Alcurus, Hodgs. - 670. Prosecusa, Reich. 671. Ixidia, Hodgs. - 672. Meropixus, Bp. -673. Ixocherus. Bp. - 674. Sphagias, Cab.

b. IIypsipeteac. - 675. Hypsipetes, Vig. - 676. Ixocincla, Hodgs. — 677. Hemixus, Hodgs. - 678. Galgulus, líitlitz.679. Microscelis, $G r$.

c. Crinigere - -680 . Ixonotus, Verr. -681 . Andropodus, $S w$. -682. Criniger, Temm. - 683. Iole, Blyth. - 684. Trichophoropsis, Bp. - 683̈. Setornis, Less. - 686. Trichixos, Less.

6 \%. Leiotrichins. . . . . . . . As. centr, et m.

a. Leiotrichese. - 687. Lioptilus, Cab. - 688. Leiothrix, Sw. - 689. Fringilliparus, Hodgs. - 690. Hemiparus, Hodgs. 691. Minla, Hodgs. - 692. Proparus, Hodgs. - 693. Sylviparus, Burton. -- 694. Suthora, IIodgs. - 695. Conostoma, Gr. - 696. Heteromorpha, Hodgs. - 697. Paradoxornis, Gould.

68. Timalind. . . . . . . . . . Asia m. Oc.

a. Timalieae. - 698. Timalia, Horsf. - 699. Chrysomma, Hodgs. - 700. Mixornis, Hors/. - 701. Macronus, Jard. 702. Myiolestes, Miull. - 703. Napothera, Boie. - 704. Laniellus, Sw.

b. Cateopittear. - 705. Turdinus, Blylh. - 706. Cacopitta, Bp. -- 707. Turdirostris, IIey.—708. Pellorneum, Sw.-709.Cinclidium, Blylh. - 710. Drymocataphus, Bl.-711. Brachypteryx, Horsf. - 712. Alcippe, Blyth. - 713. Stachyris, Hodgs. - 714. Trichostoma, Bl. - 718. Erpornis, Hodgs. 716. Malacopteron, Eylon.

c. Certhipareare. - 717. Clitonyx, Reich. - 718. Certhiparus, Less. 
CONSPECTLS SYSTEMATIS ORNITHOLOGIA.

721. Presbys, Cab. - 722. Pheugopodius, Cab. - 723. Cyphorinus, Cab. - 724. Thryothorus, Vieill. - 725. Telmatodytes, Cab.-726. Troglodytes, Vieill._727. Cistothorus, Cab.

21. CERTHIIDE.

70. Certhines.

a. Certhiere. - 728. Certhia, L. - 729. Caulodromus, Gr.

b. Tiehodromere. -730 . Salpornis, Gr. - 731. Tichodroma, Ill. - 732. Climacteris, Temm.

71. Sirtine. Cosmopol. 25

733. Callisitta, Bp. - 734. Dendrophila, Sw. - 735. Sitta, L.736. Sittella, $S w .-737$. Acanthisitta, Lafr.

22. PARIDE.

72. PARINE.

Cosmopol. 60

a. Pareae. -738. Bacolophus, Cab. - 739. Lophophanes, Kaup. -740. Machlolophus, Cab. - 741. Melanoparus, Bp.—742. Parus, L.- 743. Cyanistes, Kaup. - 744. Penthestes, Reich. -745. Pæcila, Kaup. - 746. Egithaliscus, Cab.-747. Psaltriparus, Bp. -748 . Psaltria, Temm. - 749. Mecistura, Leach. -750 . Oritiscus, $B p$.

b. Egithaleae. - 751. Panurus, Kaup. - 752. Egithalus, Vig. - 753. Anthoscopus, $C a b$.

73. Pardalotine.

As. Ocean.

754. Pardalotus, Vieill. - 755. Triglyphidia, Reich. - 756. Piprisoma, Blylh. - 757. Smicrornis, Gould.

74. Reguline.

Eur. As. Afr. Am. 10

758. Regulus, Vieill. - 759. Reguloides, Blylh.- i60. Cephalopyrus, $B p$.

23. CINCLID E.

73. Cinclina.

Eur. As. Amer.

761. Cinclus, Bechst. - 762. Ramphocinclus, Lafr. - 763. Cinclops, $B p$.

76. EuPETINE.

As. Ocean.

764. Eupetes, Temm. - 765. Grallina, Vieill. - 766. Henicurus, Temm. - 767. Ephthianura, Gould.

Extr. des Annalifs des sgienges naturelles. 
24. MOTACHLLID.

77. Motacilline. . . . . . . Eur. As. Afr. Ocean. 25

768. Motacilla, L. - 769. Nemoricola, Blyth. - 770. Pallenura, Pall. - 771. Budỵtes, Cuv.

78. Axtmine. . . . . . . . . . . . Cosmopol. 40

772. Nacronyx, $S w$ - 773. Corydalla, Vig. - 774. Agrodroma, Sw. - 77\%. Anthus, Bechst. - 776. Pipastes, Kaup. $776^{a}$. Cynadium, Sund.

25. ALAUDIDE.

79. Pyrrhulaudine.

As. Afr.

777. Pyrrhulauda, Smilh.

80. Alacding. . . . . . . . . . Cosmopol. 60

a. Callandrellea - 778. Otocorys, Bp.-779. Calandrella, Kaup.

b. Mandeae. - 80 . Rhamphocorys, Bp.-781. Melanocorypha, Boie. - 782. Mirafra, Horsf. - 783. Megalophonus, Gr. 784. Annomanes, Cub. - 785. Alauda, L. - 786. Lululla. Kaup. - 787. Galerida, Boie. - 788. Certhilauda, Sw. 789. Alemon, Keys, et Bl.

Stirps 4. CURVIROSTRES.

226. EPIMACHIDE.

81. Eрimacuine.

Oceania. 6

790. Epimachus, Cuv. - 791. Ptilorhys, Sw. - 792. Craspedophora, Gr. - 793. Seleucides, Less.

27. PARADISEIDE.

82. Paradiseinde.

Oceania. 9

794. Cicinnurus, Vieill. - 795. Paradisea, L. - 796. Xanthomelus, Bp. - 797. Diphyllodes, Less. - 798. Lophorina, Vieill. - 799. Parotia, Vieill.

83. Astrapina:.

Oceania.

800. Astrapia, Vieill. - 801. Paradigalla, Less.

8 '. Phoviganiva.

Oceania. 3

802. Plionygama, Less.

28. GLAUCOPIDE.

85. Gilaucorin.s.

Oceania.

803. Corcorax, Less. - 804. Glaucopis, Gm. - 805. Neomorpha, Cionld. - 806. Creadion, Vicill. 
29. MELIPHAGID玉.

86. Meliphagine.

As. in. Oc

807. Tropidorhynchus. Vig. -808 . Leptornis, Hombr. 809. Xanthotis, Less. - 810. Moho, Less. - 811. Entomyza, Siv. - 812. Acanthogenys, Gould. - 813. Prosthemadera, Gr. -814. Anthochæra, Vig.-815. Anellobia, Caban.-816. Manorhina, Vig. -- 817. Foulehajo, Reich. - 818. Sericulus, Sw. - 819. Meliphaga, Lewin. - 820. Hypergerus, Reich. 821. Lichenostomus, Cab. - 822. Pogonornis, Gr. - 823. Anthornis, Gr. - 824. Ptilotis, Sw. - 825. Lichmera, Cab. 826. Meliornis, Gr. - 827. Glyciphila, Sw. - 828. Entomophila, $G^{r}$. - 829. Conopophila, Reich.

87. Melithreptine.

Oceania.

830. Plectrorhyncha, Gould. - 831. Melithreptus, Vieill. -832. Hæmatops, $B$ p. -833 . Eidopsarus, Sw.

88. MYZOMELIN.

Oceania.

$1 \ddot{3}$

834. Acanthorhynchus, Gould.-835. Myzomela, Vig.-836. Cissumela, Bp. - 83\%. Certhionyx, Less.

30. ARACHNOTHERID年.

89. Arachnotherine.

Oceania.

838. Arachnothera, Temm.

31. PHYLLORNITHIDE.

90. Рhyllornithin..

As. m. Oc.

30

839. Philopitta, Is. Geo//r.-840. Phyllornis, Boie.-84.1. Yuhina, Hodgs. - 842. Mizornis, Hodgs. - 843. Ixulus, Hodgs. 84 . Jora, Hors.

91. Zosteropine.

Afr. As. Oc.

845. Zosterops, Vig. - 846. Malacirops, $B p$. -817 . Cyclopterops. $B p .-848$. Orosterops, $B p$.

32. NECTARINIIDE.

92. Prilotunina. .

Africa.

849. Piloturus, $S w$.

93. Nectarinine.

As. Afr. Oc.

850. Nectarinia, Ill. - 851. Arachnethra, $C a b$. -852 . Cinnyris,

Cuv. - 853. Adelinus, Bp. - 854. Anthodiæta, Cab. 855. Mangusia, Bp. - 856. Anthobaphes, Cab. - 857. Panæola, $C a b .-858$. Hedidypna, $C a b,-809$. Leptocoma, Cab. -860. Ethopyga, Cab.-861. Chalcoparia, Cub.-862. Chalcostetha, $C a b .-863$. Cyrtostomıs, $C a b$. 
94. Anthreptine.

As. Ocean. 20

864. Anthreptes, Sw. - 865. Cinnyricinclus, Less.

\section{DREPANIDE.}

95. Drepanina.

As. Ocean.

866. Drepanis, Temm. - 867. Himatione, Caban. - 868. Hemignathus, Licht.

\section{3 i. DICAIDE.}

96. Dic

As, Ocean.

869. Dicæum, Cuv. - 870. Prionochilus, Strickl. - 871. Pachyglossa, Ilodgs. - 872. Myzanthe, Hodys.

\section{CAREBIDA.}

97. Carebina.

A merica.

873. Cæreba, Vieill. - 87 í. Diglossa, Wagl.

98. DaCnidine.

America.

875. Certhiola, Sundev. - 876. Dacnis, Cuv. - 877. Conirostrum, $\mathrm{Or} b$.

\section{Stirps 5. DENTIROSTRES.}

\section{LANIIDA.}

99. Malaconotiñ.

Africa. 60

a. Vangeac. - 878. Vanga, Vieill. - 879. Xenopirostris, Bp. - 880. Artamia, Lafr. - 881. Archolestes, Cab.

b. Malacomotcate. - 882 . Chlorophoneus, Cab. - 883 . Pelicinius, Boie. - 884. Laniarius, Boie. - 883. Telephonus, Sw.

- 886. Harpolestes, Cub. - 887. Malaconotus, Sw. 888. Chaunonotus, Gr. - 889. Hapalophus, Gr.-890. Rhynchastatus, Br. - 891. Dryoscopus, Boie. - 892. Nilaus, Sw. - 893. Calicalicus, Bp.

100. Prionorin.s.

Afric. Ocean.

894. Lurocephalus, Smilh.-895. Sigmodus, Temm.-896. Prionops, Vieill. - 897. Fraseria, Bp. - 898. Tephrodornis, Sw. - 899 . Cabanisia, Bp.

a. Convinelle:- - 900. Urolestes, Cab-_-901. Corvinella, Less.

h daniear. - 902. Janius, L. - 903. Fiscus, Bp. - 904. Col- 
lurio, Bp. - 905. Otomela, Bp. - 906. Phoneus, Bp.907. Leucometopon, Bp. - 908 . Enneoctonus, Boie.

102. Pachicephaline.

As. Oceania.

909. Colluricincla, Vig. - 910. Rectes, Reich. - 911. Falcunculus, Vieill. - 912. Pteruthius, Sw. - 913. Allothrius, Temm. - 914. Pucherania, Bp. - 915. Pachycephala, Sw. 916. Timixos, Blyth.-917. Psaltricephus, Bp.-918. Eopsaltria, Sw. - 919. Hyloterpe, Cab.

103. Vireoniñ.

America.

920. Cyclorrhis, Sw. - 921. Vireolanius, Dubus. - 922. Vireo, Vieill. - 923. Vireosylvia, Bp. - 924 . Hylophilus, Temm.

\section{ARTAMIDE.}

104. Artamine.

Asia m. Afr. Oc. 18

925. Artamus, Vieill. - 926. Ocypterus, Cuv. - 927. Leptopterus, $B p .-928$. Cyanolanius, $B p .-929$. Tephrolanius, $B p$.

105. A nalcipodin.t.

Asia m. Oc. Madag. $\ddot{3}$

930. Analcipus, $S w .-931$. Anais, Less. - 932. Psaropholus, Jard. - 933. Oriolia, Is. Geoffr.

38. ORIOLIDE.

106. Orioline.

Eur. As. Afr. Oc.

934. Oriolus, L. -935 . Galbulus, $B p .-936$. Broderipus, $B p$. - 937. Baruffius, Bp. - 938. Xanthonotus, Bp. - 939. Mimeta, Vig. - 940. Sphecotheres, Vieill.

39. EDOLIID 2 .

107. Edolinas.

As. Afr. Oc.

941. Chibia, Hodgs. - 942. Balicassius, Bp. - 943. Dicranostreptus, Reich. - 944. Edolius, Cuv.-940̈. Bhringa, Hodgs. -946. Chaptia, Hodgs. -947. Dicrourus, Vieill.-948. Drongo, Less. - 949. Musicus, Reich. - 950. Buchanga, Hodgs. - 951. Irena, Horsf. - 952. Prosorinia, Hodgs. - 953. Edolisoma, Pucheran.

108. Ceblepyrine.

As. Afr. Oc. 60

954. Pteropodocys, Gould - 955. Graucalus, Cuv. - 956. Campephaga, Vieill. - 957. Oxynotus, Sw. - 958. Ptiladela, Pucheran. - 959. Ceblepyris, Cuv. - 960. Volvocivora, Hodgs. - 961. Lanicterus, Less. - 962. Lobotos, Reich. - 963. Symmorphus, Gould.- 964. Lalage, Boie.-965. Pericrocotus, Boie. 
40. AIIPELIDA.

109. Anpelink. . . . . . . . . Eur. As Afr. Am.

966. Ampelis, L. - 967. Hypocolius, Bp. - 968. Lepturus, Less. - 969. Ptilogonys, Sw. - 970. Cichlopsis, Cab. 971. Myiadestes, Sw.

\section{NIUSCICAPID E.}

110. Monarchine . . . . . . . . As. Afr. Oc.

972. Xenogenys, Cab. - 973. Melænornis, Gr. - 974. Melanopepla, Cab. - 975. Chasiempsis, Cab.-976. Metabolus, Bp.97\%. Pomarea, Bp.-978. Piezorhynchus, Gould.-979. Symposiachrus, Bp. - 980. Monarcha, Vig. - 981. Arses, Less. - 982. Philentoma, Eyton. - 983. Anthipes, Blyth. 984. Dimorpha, Hodgs. - 995. Ochromela, Blyth.

111. Muscicapine. . . . . . . . . Rur. As. Afr. Oc. 50 986. Glaucomyias, Cab. - 987. Cyanoptyla, Blyth. - 988. Eumyias, Cab. - 989. Cyornis, Blyth. - 990. Hemipus, Blyth. - 991. Hemichelidon, Boie. - 992. Muscicapula, Blyth. 993. Nuscicapa, L. - 994. Butalis, Boie. - 995. Micræca, Gould. - 996. Alseonax, Cab. - 997. Charidhylas, Bp.998. Erythrosterna, Bp. - 999. Xanthopygia, Blyth.

112. Mylagrine. . . . . . . . Afr. As. Oc. Am. 80

1000. Terpsiphone, Gloger. - 1001.Tschitrea, Less. - 1002.Muscipeta, Cuv. - 1003. Trochocercus, Cab. - 100k. Elminia, Bp. -1003. Rhipidura, Vib.-1006. Leucocerca, Sw.--1007. Sauloprocta, Cab.-1008. Seisura, Vig.-1009. Cryptolopha, Sw. - 1010 . Chelydorynx, Hodgs. - 1011. Myiagra, Vig. 1012. Bias, Less. - 1013. Hypothymis, Boie.- 1014. Hyliota, Sw. - 1015. Platystira, Jurd. - 1016. Muscisylvia, Less. - 1017. Todopsis, Bp. - 1018. Stenostira, Bp. 1019. Culicivora, Sw.

12. TINAGRIDE

113. TaCHYPHOXIN.E.

America. $\quad 50$

a. Hamphocellere. - 1020. Sericossypha, Less. - 1021. Lamprotes, B1. - 1022. Ramphocelus, Desmar. - 1023. Jacapa, Iip. - 1024. Ramphopis, Vieill.

b. 'achyphone:ac. - 1025. Pyranga, rieill. - 1026. Phanicothraupis, Caban. - 1027. 'Tachyphonus, Vieill. - 1028. Trichothraupis, Cab. - 1029. Lanio, Vicill. - 1030. Comarophagus, B1. - 1031. Icteria, Vicill. - 1032. Orthogonys, Striclil. - to3:3, Cyanicterms, Br. 
114. Tanagrine.

America. 100

c. Tanagrea.-103k. Buthraupis, Cab.-1035. Dubusia, Bp. 1036. Tanagra, L._-1037. Spindalis, Jard.-1038. Anisognathus, Reich. - 1039. Stephanophorus, Strickl. - 1040. Iridornis, Les.s.

d. Callisteae. - 1041 . Callispiza, $B p$. - 1042. Chalcothraupis, Bp. - 1043. Tatao, Bp.-1044. Thraupis, Bp.-1045. Calliparæa, Bp. - 1046. Chrysothraupis, $B p$. - 1047. Calliste, Boie. - 1048 . Ixothraupis, Bp. - 1049. Gyrola, Reich. 1050. Tanagrella, Sw.

115. Euphonine.

America. $\quad 30$

e. Euphonea - 1051 . Tersina. Vieill. - 10 ב̈. Pipreola, Sw. - 1053. Procnopis, Cab. - 1054. Cyanophonia, Bp. 1055. Chlorophonia, Bp.-1056. Ypophæa, Bp. - 1057. Pyrrhuphonia, Bp. - 1058. Acroleptes, Schiff. - 1059. Euphona, Desm. - 1060. Iliolopha, $B p$.

116. Sylvicoline.

America. 100

f. Nemosicae.-1061? Egithina, Vieill. -106\%. Nemosia, Vieill. - 1063. Hemithraupis, Cab. - 1064. Granatellus, Bp. 1065. Cardellina, $B p$.

g. Helmitherear. - 1066. Helminthophaga, Cab. - 1067. Helmintheros, $R a f$.

h. Setophagere. - 1068. Basileuterus, Cab. - 1069. Setophaga, Sw. - 1070. Myiodioctes, Aud. - 1071. Euthlypis, Cab.

i. ylvicoleas. - 1072. Seiurus, Sw.--1073. Sylvicola, Sw. 1074. Pachysylvia, Bp. - 1075. Thaumasioptera, Schiff. 1076. Mniotilta, Vieill. - 1077. Rhimamphus, Rafin.1078. Myiothlypis, Cab. - 1079. Parula, Bp. - 1080. Trichas, Sw.

Stirps 6. FISSIROSTRES.

\section{HIRUNDINIDE.}

117. Hirundininæ.

Cosmop.

a. Hirandineae. - 1081. Hirundo, L. - 1082. Cecropis, Boie. - 1083. Uromitus, Bp. - 1084. Atticora, Boie.

b. Prognear. - 1085. Progne, Boie. - 1086. Petrochelidon, Cab.-1087. Tachycineta, Cab.-1088. Psalidoprocne, Cab.1089. Ptyonoprogne, Reich. - 1090. Cheramoeca, Cab. 1091. Cotyle, Boie. - 1092. Chelidon, Boie. 


\section{Tribus II. VALUCRES.}

\section{Cohors I. Z YG ODACT I LI.}

Stirps. 7. AMPHIBOLI.

\section{RHAMPHASTIDA}

118. Rilamphastine.

Anier. calid. 50

1093. Rhamphastos, L. - 1094. Pteroglossus, IIl. - 1095. Andigena, Gould. - 1096. Selenidera, Gould. - 1097. Aulacoramphus, Gr. - 1098. Beauharnesius, Bp.

\section{5้. CUCULIDE.}

119. Scythropina.

Ocean.

1099. Scythrops, Lath.

120. Phenicopheixe.

Amer., Afr., Ocean.

1100. Carpococcyx, Gr. - 1101. Rhinortha, Vig. - 1102. Dasylophus, Sw. - 1103. Lepidogrammus, Reich. - 110\&. Phænicophæus, Vieill. - 1105. Melias, Glog. - 1106. Zanclostomus, Siv.

121. Crotophagina.

Amer. trop.

6

1107. Crotophaga, $L$.

122. Centropodine . . . . . . . As., Afr., Oc.

1108. Taccocua, Less. - 1109. Centropus, Ill. - 1110. Coua, Cuv. - 1111. Serisomus, Sw.

123. Saurotherine.

1112. Saurothera, Vieill. - 113. Geococcyx, Wagl.

124. Coccyzine.

1114. Cultrides, Pucheran. - 1115. Diplopterus, Boie. 1116. Ptiloleptis, Sw. - 1117. Guira, Less. - 1118. Piaya, Less. - 1119. Coccyzus, Vieill. - 1120. Dromococcyx, Wagl.

125. Cucculinx. Eur., As., Afr., Oc. 50

1121. Eudynamis, Vig. - 1122. Oxylophus, Sw. - 1123. Cuculus, L. - 1124. Cacomantis, Miill. - 1125. Hierococcyx, Mull. - 1126. Surniculus, Less. - 1127. Lampromorpha, Vig. 1128. Chrysococcyx, Boie. - 1129. Chalcites, Less. 


\section{Stirps 8. SCANSORES.}

46. PICIDE.

127. Picine.

a. Picex. - 1131. Dryotomus, Sw. - a. Megapicus, Malh. - b. Diyolomus, Sw. - c. Campephilus, Gr. - ${ }^{*} 1132$. Reinwardtipicus, Bp. - 1133. Hemilophus, Sw. - *a. Mulleripicus, Bp. - ${ }^{*}$ b. Lichtensteinipicus, Bp. - c. Hemilophus. Bp. 1134. Dryocopus, Boie. - 1133. Dryopicus, Malherbe. *1136. Pilumnus, Bp. - 1137. Picus, L. - *a. Dyctiopicus, Bp. - * b. Phrenopicus, Bp. - ${ }^{*}$ c. Trichopicus, Bp.— *d. Pipripicus, Bp.- e. Picus, Bp. - *f. Hypopicus, Bp. - *g. Leiopicus, Bp. - *h. Yungipicus, Bp. - 1138. Picoides, Lacép. a. Apternus Sw. - b. Tridactylia, Stephens.

b. Celere. - 1139. Celeus, Boie.- 1140 . Cerchneipicus, Bp. *1141. Blythipicus, Bp. - 1142. Micropternus, Blyth.1143. Neiglyptes, $S w .-1144$. Hemicercus, $S w$.

c. Chrysoptileae. - + Africance. - 114 5. Dendropicus, Malh. - 1146. Campethera, Licht. - 1147. Mesopicus, Mall. - 1148. Dendromus, Sw. - *149. Pardipicus, Bp.

if Americance. -1150 . Chrysoptilus, Sw. - 1151. Chrysopicus, Malh. - 1152. Chloronerpes, Sw. - 1153. Veniliornis, Bp. - * 1154 . Capnopicus, Bp.

d. Tigeae. -1135 . Chrysocolaptes, Blyth. -1136 . Brachypternus, Strikl. - 1157. Tiga, Kaup. - 1158. Chrysonotus, Sw. - 1159 . Gecinulus, Sw.

e. Gecimese. - 1160. Gecinus, Boie. - 1161. Chrysophlegma, Gould. - 1162. Brachylophus, Strickl. - 1163 . Callipicus, Bp.

f. Centurese. - 1164. Leuconerpes, $S w$. - 1163. Melanerpes, Sw. - 1166 . Tripsurus, Sw. — 1167 . Xiphidiopicus, Bp. 1168. Centurus, Sw.—* 1469 .Zebripicus, Malh.-1170. Linnæipicus, Mall.

g. Colapteae. - 1171. Colaptes, $S w$. - 1172 . Pituipicus, Bp. - 1173. Geocolaptes, Burch. — *1174. Hypoxanthus, Bp. - 1175. Malherbipicus, $B p$.

128. Yungine

Eur. As. Afr. 1176. Yunx, $L$.

129. Picuminas. As. m. Oc. Am. m. 15

1177. Picumnus, Temm.-1178. Piculus, Is. Geo/fr.-1179. Microcolaptes, Gr. - 1180. Asthenurus, Sw. - 1181. Vivia, Hodgs. - 1182. Sasia, Hodgs.

Extr. des Antales des sciences naturelles. 
47. BUCCONIDE.

\section{Stirps 9. BARBATI.}

130. Bugconine.

As. Afr. Oc. Am. m.

1183. Pogonias, Ill. - 1i84. Læmodon, Gr. - 1185. Gymnobucco, Bp.-1186. Xylobucco, Bp. 一 *18\%. Tricholæma, Verr.-1488. Trachyphonus, Ranzani.-1489. Barbatula, Less. - 1190. Psilopogon, Mull. (Buccotrogon, Reich.) - 1191. Psilopus, Temm.-1192. Bucco, L.-1193. Negalæma, Gr.- - Chotorea, Bp.- b. Mlegalema, Bp. - c. Cyanops, Bp.-d. Xantholcema, Bp.-1194. Micropogon, Temm. - 1193. Eubucco, Bp.

4. CAPITONIDE.

131. Capitonina.

Amer. m. 30

1196. Capito, Temm. - 1197. Chaunormis, Gr. - 1198. Tamatias, Cuv. - 1193. Nyctastes, Strickl, - 1200. Malacoptila, Gr. - 1201. Scotocharis, Gloger.-1202. Monasa, Vieill. - 1203. Chelidoptera, Gould.

49. LEPTOSOHIDA.

132. Leptosonina. Madagascar. 1

1204 . Leptosoma, Sw.

50 . GALBULid 2 .

133. Galbulink.

Amer. m. 16

4205. Jacamerops, Cuv. - 1206. Galbula, L. - *1207. Urogalba, Bp. - *1208. Brachygalba, Bp. - 1209. Gabalcyrhynchus, O. des Murs. - 1210. Jacamaralcyon, Cuv.

31. TROGONIDE.

Stirps 10. HETERODACTYLI.

134. Trogoninz.

Amer. calid. As. Afr. Oc. 48

1211. Calurus, Sw.-a. Pharomachrus, De la Llave.-b. Cosmurus, Reich.—1212. Trogon, L.- a. Trogon, L.- b. Curucujus, Bp. - c. Trogonurus, Bp.—d. Temnotrogon, Bp. - 1213. Priotelus, Gr.-1214. Apaloderma, Sw. - 1215. Harpactes, Sw. - a. Ilarpacles, Bp. — - * b. Duvancelius, Bp. — * *. Pyrotrogon, Bp. - * d. Orestios, Bp. - * e. Apalharpactes, Bp.

Cohors 2. ANISODACTYLI.

¿2. BCCRROTIDA.

Stirps. 11. FRUGIVORI.

13:3. Buclinotriz. .

As. Afr. Ocean.

a. Sucorveas. - 1216. Bucorvus, Bp.

b. IBuceroteax. - *1217. Ceralogymna, Bp. - 1218. Tmetoceres, Cab. - 1219. Berenicornis, Bp. - 1220. Buceroturus, 
Bp. (Cranocerus, Reich.) - 1221. Buceros, L.— *1222. Homraius, Bp. - 1223. Hydrocorax, Briss. - 1224. Hydrocissa, Bp. - a. Anthracoceros, Reich. - b. Anorrhinus, Reich. 1225. Rhyticeros, Reich. - a. Aceros, Hodgs. - b. Cassidix, Bp. - c. Rhyticeros, Bp.

c. 'Tockere. - 1226. Calao, Bp. - 1227. Penelopides, Reich. - 1228. Meniceros, Glog. - 1229. Rhinoplax, Gloger. 1230. Grammicus, Reich. - 1231. Rhynchoceros, Glog. 1232. Tockus, Less.

136. Eurycerotine. . . . . . . . . . Madagascar.

1233. Euryceros, Less.

53. MUSOPHAGID E.

137. Musophagine.

Africa. 14

1234. Musophaga, Isert. - 1235. Gallirex, Less. - 1236. Turacus, Cuv. - 1237. Opæthus, Vieill. - 1238. Corythaix, Ill. 1239. Coliphimus, Smilh. - 1240. Schizorhis, Wagl.

54. OPISTHOCOMIDE.

138. Оріsтносомгіє. . . . . . . . . Am. merid.

12 1. Opisthocomus, Vieill.

55. COLIIDE.

139. Colinne.

Africa. 6

*1242. Urocolius, $B p$. - 1243. Colius, Br. - *1244. Rhabdocolius, $B p$.

56. PHYTOTOMIDE.

140. Рнятотоиine. . . . . . . . . . . Amer. merid.

1245. Phytotoma, Molina.

Stirps 12. FORMICIVORI.

57. MENURID正.

14. Menurine.

Australasia.

2

124.6. Menura, Davis.

14.2. Orthonychine.

1247. Orthonyx, Temm.

58. MYIOTHERIDE.

143. Myiotherine. . . . . . . . . . Amer. merid.

a. Hylaeteae. - 1248. Hylactes, King. - 1249. Pteroptochus, Killl. - 1250. Rhinocrypta, Gr. - 1251. Triptorhinus, Caban. - 1252. Sarochalinus, Caban. - 1253. Scytalopus, Gould. 1254. Sylviaxis, Less.

b. Myiothereae. - $125 \%$. Grailaria, Vieill. - 1256. Colobathris, Bp. ex Cab.-1257. Hipsybemon, Cab.-1258. Chamæza, Vig. 
1239. Holocnemis, Strickl. - 260 . Hypocnemis; Cuban. 1261. Nyiothera, III. - 1262. Drymophila, Sw. - 1263. Pithys, Vieill.-1264. Gymnopithys, Schiff.-1265. Corythopsis, Sundev. - 1266. Conopophaga, Vieill. - 1267. Pyriglena, Caban. - 1268. Chamæa, Gambel.

14. Thancophulixe. . . . . . . . Amer. merid.

a. Whamaphileas.-1269. Thamnophilus, Vieill.-1270. Cymbilaimus, Gr. - 1271. Diallastus, Reich-1272. Nisius, Reich. - 1273. Batara, Less. - 1274. Taraba, Less.

b. Dasycephneare. - 1275. Agriornis, Gould. - 1276. Dasycephala, Sw.-127\%. Attia, Less.-1278. Dysithamnus, Cab. -1279. Thamnolanius, Less.

c. Forraicivorege. - 1280. Formicivora, Sw. - 1281. Rhopoterpe, Caban. - 1282. Herpsilochmus, Cab. - 1283. Ellipura, Cab. - 1284. Tanidiura, Reich. - 1285. Thamnomanes, Cab. 1286 . Rhamphocænus, Vieill. (Acontistes, Sundev.)

89. ANABATIDA.

$1 \%$ O. Axabatinas. Amer. merid.

128\%. Anabates, Temm. - 1288. Cichlocolaptes, Reich.1289. Automolus, Reich. - 1290. Homorus, Reich.1291. Pseudoseisura, Reich.-- 1292. Siptornis, Reich.1293 . Lepioxyara, Reich.

146. Sxxallacina.

Am. m. 100

1294. Phacellodromus, Reich. - 1295. Astheres, Reich. 1296. Cranioleuca, Reich.-1297. Oxyurus, Sw. - 1298. Molanopareia, Reich.-1299. Bathmidura, Cab-1300. Anumbius, Less. - 1301. Leptostenura, Reich.-1302. Synallaxis, Vieill. - 1303. Sylviorthorhynchus, O.des Murs.

147. Furamina.

Am. m. 100

130 4. Philydor, Less. - 1303 . Heliobletus, Reich. - 1306. Rhodinocycla, IIarll. - 1307. Furnarius, Vieill. - 1308. Ochetorhynchus, Meyen.-1309. Henicornis, Gr.-1310. Limnornis, Gould. - 1311. Thelydrias, Reich. - 1312. Tartarea, Reich. 1313. Cinclodes, Gr. - 1314. Opetiorrhynchus, Temm. 131\%. Cillurus, Reich.-1316. Upucerthia, Goffr.-1317. Cinclocerthia, Gr. - 1318. Gcobates, Sw. - 1319. Geositta, Sw.

148. Xaxopixi:

Amer. m.

1320. Xenops, Illig. - 1321. Anabaxenops, Lafr. - 1322. Thripophaga, Caban. - 132.). Pseudocolaptes, Reich. - 132. Margrarornis, Reich. - 1325. Sclerurus, Sw. - 1326. Lochmia, Sw. - 1327. Pygarthicus, Licht. (Dendrodromus, Gould. - Dromode:dron, Gr.) - 1328. Oxyramphus! Sw. 
60. DENDROCOLAPTIDE.

14.9. Dendrocolaptina.

Amer. cal.

a. Dendrocolapteae. - 1329. Dendrocolaptes, Herm. b. Xiphocolaptes, Less. - 1330. Cladoscopus, Reich. 1331. Lepidocolaptes, Reich. - 1332. Picolaptes, Less. *1333. Dacryophorus, Bp. - 1334. Xiphorhynchus, Sw.1335. Drymornis, Less. - 1336. Nasica, Less. - 1337. Dendrornis, Eyt.-1338. Dendroplex, Su. - 1339. Neops, Vieill. (Glyphorhyrchus, Wied.) - 1340. Sittasomus, Sw.

b. Dendrocopeae. - 134.1. Dendrocops, Sw. - 1342. Dendrocincla, Gr. - 1343. Dendroxetastes, Eyt.

Stirps 13. MUSCIVORI.

61. TODIDA.

150. Tenropterisk. . . . . . . Amer. mer. et centr. 100

a. Mlectorareac. - 1344 . Alectorurus, Vieill. -1345 . Gubernetes, Such.

b. Tanioptereae. - *1346. Ixoreus, Bp. - 1347. Tænioptera, Bp. - 1348. Pepoaza, A sara. - 1349. Ochthæca, Cab. 1350. Machetornis, Gr. - 1351 . Suiriria, Reich. - 1352. Fluvicola, Sw. - 1353. Myiophila, Reich. - 1354. Muscisaxicola, Lafr. (Ptyonura. Gould.) - 1355. Cnipolegus, Boie.-1356. Ada, Less. - *1357. Hydrozetetes, Schiff. - 1358. Lichenops, Commerson. - 1359. Muscigralla, Lafr. (Ochthites, Caban.) - 1360. Euscarthmus, Wied. - 1361. Colopterus, Cab. - 1362. Centrites, Cab. - 1363. Hapalicercus, Caban. 136\%. Myiosympotes, Reich. - 1365. Cyanotis, Sio. 1366. Anairetes, Caban.

c. Platyrhynchexe. - 1367. Todirostrum, Less. - 1368. Serpophaga, Gould. - 1369. Phoneuticus, Cab. - 1370. Platyrhynchus, Desmar.

151. Tyrannine. . . . . . . . . . . . . America 250

a. Milvuleae. - 1371. Copurus, Strickl. - 1372. Milvulus, Sw. - 1373. Despotes, Reich. - 1374. Muscipipra, Less.

b. Tyrannese. - 1375. Scaphorhynchus, Wied. - 1376. Saurophagus, Sw. - 1377. Dioctes, Reich.-1378. Satellus, Reich. - 1379. Tyrannus, Vieill. - 1380. Lophyctes, Cab. 1381. Onychopterus, Reich. - *1382. Myiodynastes, Bp. 1383. Arundinicola, Lafr.— 1384 . Syrichta, Bp. -1385. Tyrannula, Sw. — * 1386 . Capnixus, Bp. — * 1387. Sayornis, Bp. — ${ }^{*}$ 388. Kaupornis, Bp. - 1389. Myiarchus, Bp. 1390. Myiacleptes, Caban - 1391. Pyrocephalus, Gould. - 
*1392. Myiozeta, Schiff. - *1393. Sericoptila, Schiff. -1394. Elænia, Sundeval. - 1393. Leptopogon, Caban.1396. Hirundinea, Orb. - 1397. Myionectes, Caban. 1398. Hapalura, Caban. - 1399. Tyrannulus, Vieill.

c. Cychnorhymeheae. - * 1400 . Pipromorpha, Schiff. *1401. Myiocapta, Schiff. - 1402. Myiobius, Gr.-1403. Megalophus, Sw. - 1404. Onychorynchus, Fisch. - 1405. Cychlorhynchus, Sundev.

152. TODINE.

Amer. calid.

1406. Todus, L.

153. Psarine. Amer. calid. 25 1407. Tityra, Vieill.-*1408. Exetastus, Bp. - 1409. Erator, Kaup. - 1410. Pachyramphus, Gr. - *1411. Platypsaris, Bp. - 142. Psaris, Cuv. - 1413. Chloropsaris, Kaup. *14 14. Callopsaris, $B p$.

62. COTINGIDE.

15\%. Lipadgixis. . Amer. merid. 9

4415. Lipaugus, Boie. - 1416. Lathria, Sw. — ${ }^{*} 1417$. Aulea, Schiff.- * 14 18. Schiffornis, Bp.

135. QUeruline.

Amer. merid. $\ddot{3}$

1419. Pyroderus, Gr. -1420. Querula, Vieill. — *1421. Hæmatoderus, $B p$.

13̋. Gymoderind. . . . . . . . . . . . Amer. merid. 8

1422. Gymnocephalus, Geoffr. - 1423. Cephalopterus, Geoffr. 1424. Gymnoderus, Geoffr. - 1425. Ch asmorhynchus, Temm. - 1426. Arapunga, Less.

157. Cotingina.

Amer. merid. 30

a. Cotingeae. - 1427. Pyrrhorhynchus, Lufr.-1428. Euchlornis, De Filippi-1429. Ptilochloris, Sw. -1430. Tijuca, Less. -1431. Ampelion, Caban. (Carpornis. Gr.) - 1432. Heliochera, J) Filippi. - 1433. Phibalura, Vicill. - 1434. Xipholena, Gloger. - 1433. Cotinga, Br. - * 1 136. Porphyrolæma, Bp.

b. Iodopleureare. - 1437. Iodopleura, Less.

6.3. PIPRIDE.

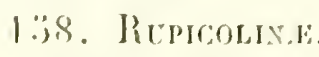

Niner. mer.

143.5. Rupicola, Arr. - 1439. Phenicocercus, Sw.

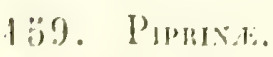

Amer. calid. 40

1440. Antilophia, Reich. (Iretopia, $S w)$.-144 . Masius, Bp. 14.2. Manacus, Mr. (Chiromacheris, Caban.) - *14.3. Heteropolua, Schiff- - 144. Xenopipo, Cubun, ${ }^{*} 14 \% 3$. Chiroprion, 
Schiff. - 446. Chiroxiphia, Caban. - 1447. Hicura, Reich. - *448. Cercophæna, Schiff. - 1449. Cirrhipipra, Bp.

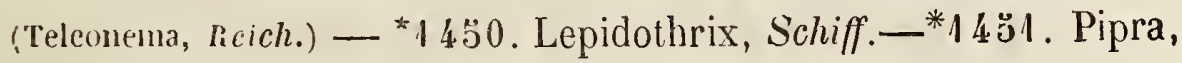
L. - 1452. Dixiphia, Reich. - *153. Ceratopipra, Bp. *1454. Corapipo, Schiff. - *1455. Dasyncetopa, Schiff. *1456. Machæropterus, Schiff. - 1457. Hemipipo, Caban. 1458. Piprites, Caban. - 1459. Calypturus, Sw.

\section{EURYLAIMID 2 .}

160. Caliptomenine.

1460. Calyptomena, Raffles.

161. Eurylamina.

Asia m. Oc.

1461 . Peltops, Wugl. - 1462. Parisomus, Sw. - 1463. Serilophus, Sw. - 1464. Cymbirhynchus, Vig. - 1460̈. Eurylæmus, Horsf. - 1466 . Corydon, Less.

162. Simthornituink.

Africa

4467. Smithornis, $B p$.

6ว. PITTID\&.

Stirps 14. CALLOCORACES (Callichromi).

163. Pittine. As. m. Afr. Oc.

1468. Brachyurus, Thunb. (Pitta, Reich.) — *a. Gigantipitla, Bp. - b. Brachyurus, Bp.—*c. Erythropitta, Bp.-**d. Iridipitta, Bp. - ${ }^{*}$ e. Melanopitta, Bp.—1469. Pitta, Vieill. (Eucycla, Reich.) 66. CORACIIDA.

164. Coracinna. . . . . . . Eur. Afr. As. m. Ocean.

*1470. Coraciura, Bp. - 1471. Coracias, L. - 1472. Colaris. Cuv. - 1473. Eurystomus, Vięill.

165. Atelornithise.

Madagascar.

14.74. Brachypteracias, Lafr. - 14.73. Atelornis, Pucheran.

67. PRIONITIDE.

166. Prionitine.

Amer. calicl. 14

1476. Crypticus, Sw. - 1477. Prionites, Ill. - 1478. Baryphonus, Vieill. - 1479. Hylomanes, Licht.

68. MEROPIDE.

Stirps 15. GRESSORII (Syndactyli).

167. Meropine. . . . . . . . . Eur. As. Afr. Oc.

a. Meropere. - 1480. Merops, L.- a. Merops, Reich. - b. Blepharomerops, Reich. - c. Erops, Reich.—*d. Urica, Bp. e. Melittophas, Reich. - 1481. Phlothrus, Reich.-1482. Melittotheres, Reich. - 1483. Tephrærops, Reich. - 1484. Melittophagus, Boie. - 1183. Sphecobus, Reich. 
b. Nyetiornitheae. - 1486. Coccolarynx, Reich. - 1487. Meropiscus, Sund.-1488. Meropogon, Bp.-1489. Nyctiornis, Sw. - a. Nyctiornis, Reich. - b. Bucia, Hodgs.

69. ALCEDINIDA.

168. Dacelonine. . . . . , . As. m. Afr. Ocean.

1490. Dacelo, Leach. - 1491. Choucalcyon, Bp. - 1492. Melidora, Less. - 1493. Syma, Less. - 1494. Paralcyon, Bp. - 1498. Lacedo, Reich. - 1496. Actenoides, Hombr. et $J$. - 149\%. Cittura, Kaup. - 1498. Chelicutia, Reich.

169. Halcrovine. . . . . . . Eur. As. m. Afr. Ocean. 60

1499. Callialcyon, $B p .-\ldots$ 1500. Cancrophaga, Bp. - 1501. Todiramphus, Less.-1502. Ispidina, $K p .-1503$. Tanysiptera, Vig. - 1504 . Halcyon, Swo- 1505 . Ramphalcyon, Bp. - a. Ramphalcyon, Reich. - b. Hylcaon, Reich.- \$506. Ceix, Lacép.

170. Alcedinines. . . . . . . . Eur. As. Afr. Am. Oc.

a. Ceryleac. - 1507. Megaceryle, Kp. — *1508. Streptoceryle, Bp. - 1509. Ceryle, Boie. - 1510. Chloroceryle, Kp. 1511 . Amazonis, Reich.

b. Alcedinese. - 1512 . Alcedo, L. - 15\%3. Corythornis, Kp. - 1514. Alcyone, Sw. (Therosa, Mull.)

\section{Stirps 16. TENUIROSTRES (Epopides).}

70. UPUPIDE.

171. UPUPiNe.

Eur. As. Afr. 与

1515 . Upupa, $L$.

\%. PROMEROPID E.

172. Falculiñ . . . . . . . Madagascar, Ins. Borb.

1 $\$ 16$. Falculia, Is. Geoffr. - 1517 . Fregilupus, Less.

173. Promeropinte. . . . . . . . . . . Africa.

1318. Promerops, Br. - 1519. Irrisor, Less. - 1520. Rhinopomastes, Smilh.

72. TROCHILIDA.

$$
\text { Stirps 1\%. SUSPENSI (Trochili). }
$$

17\%. Gruptese. . . . . . . . . . Amer. merid.

1521. Grypus, Spix. (Rhamphodon, Less.) - 1522. Myiaëtina, $B p$. (Eutoxeres, Reich.) - 1523. Glaucis, Boie. - 1524. Threnetes, Ciould. - 1525. Doleromyia, Bp. (Lencippus.)

$17 \%$. Phemonitumato.

Amer trop.

1526. Mhetornis, Sw. - 1527? Ametrornis, Reich. - 1528. Orthornis, Bp. - 1329. Guyornis, $B p$. - 1530. Pygmornis, $B p$ (Eremita, lieich.) 
176. Lampornithine.

Amer. mer. et centr.

1 3̈31. Topaza, Gr. - 13̈32. Oreotrochilus, Gould. - 1533. Eulampis, Boie. - b. Sericotis, Reich. - 1534. Lampornis, Sw. - a. Anthracothorax, Boie. - b. Hypophania, Reich. c. Floresia, Reich. - $153 \%$. Campylopterus, Sw. - a. Pampa, Reich. - b. Platystopterus, Reich. - c. Scepiopterus, Reich. - d. Jonolcema, Reich. - 1536. Aphantochroa, Gould. 1537. Petasophora, Gr. - a. Anais, Reich. - b. Delphinella, Reich. - 1538. Schistes, Gould. - 1539. Heliothrix, Boie. 1540 .Leadbeatera, $B p$. (He'iodoxa, Gould.)

177. Cunanthine. . . . . . . . Amer. mer. et centr.

a. Patagoneae. - 1541. Patagona, Gr. - 1542. Pterophanes, Gould. - 1543. Docimastes, Gould.

b. Dorifereae. - 1544 . Heliomastes, $B p$. -1545 . Ornithomyia, Bp. (Callopistria, Reich.) - 1546 . Dorifera, Gould. (Hemistephania, Reich.) - 1547. Helianthea, Gould. - b. Hypochrysia, Reich. - 1548. Bourcieria, Bp. - a. Conradinia, Reich.- b. Lampropygia, Reich. - 1549. Cœligena, Bp. (Homophania, Reich.) 1550. Lafresnaya, Bonap. - 1551. Chrysobronchus, Bonap. (Smaragdites, Reich. nec Boie.) - 1552. Heliangelus, Gould. 1553. Heliotryppha, Gould. (Parzudakia, Reich.) - 1554 . Eriocnemys, Reich. - a. Eriocnemys, Reich. - b. Aline, Reich. c. Mosqueria, Reich. - d. Luciania! Reich. - *1535. Derbyomiya, $B p$.

c. Cynantheae. - 1556 . Lesbia, Less. (Cometes, Gould. - Sappho, Reich.) - 1557. Cynanthus, Sw. (Lesbia, Reich.)

d. Metallureae. - 1558. Oxypogon, Gould. - 1559 . Lampropogon, Gould. (Chalcostigma, Reich.) - 1560. Aglæactis, Gould. - 1561. Ramphomicron, Bp. - 1562. Metallura, Gould. 1563. Myiabeillia, Bp. (Guimetia, Reich.) - 1564. Urosticte, Gould. - 1565. Augastes, Gould. - b. Lumachellus, Reich. 1566. Adelomyia, $B p$.

478. TrochiLin.e.

America. 162

a. Florisugexe. -1567 . Florisuga, $B p$. -1568 . Delattria, $B p$. (Lamprolæma, Reich.) - 1569. Clytolæma, Gould. - a. Phoeoloema, Reich. - b. Boissonneaua, Reich. - 1570. Thalurania, Gould. (Glaucopis, Boie, nec Gm.) - 1571. Eupetomena, Gould. (Prognornis, Reich.)

b. Polytmeae. - 1572 . Polytmus, $B r$.

c. Amaziliese. - 1573. Cyanomyia, Bp. (Uranomitri, Reich.) 1574. Amazilius, Bp. - 1575. Chrysuronia, Bp. - 1576. Sau- 
cerottia, Bp. - 1577 . Sporadinus, Bp. (Ricordia, Reich.) 4578. Hylocharis, Boie. (Chalybura, p. Reich.) - 1579. Chlorostilbon, Gould. - 1580. Thaumantias, Bp. - a. Thaumantias, Reich. - b. Leucochloris, Reich. - c. Margarochrysis, Reich. - 1581. Juliamyia, Bp. - 1582 . Sapphironia, Bp.a. Basilimna, Reich. ex Boie. - b. Cyanochloris, Reich.

d. Avoeetulear. - 1583. Avocettula, Reich. - 1584 . Avocettinus, $B p$.

e. Trochileae. - 1580 . Sephanoides, Less. - a. Eustephanus, Reich. - b. Stokoesiella, Reich. - 1386 . Chrysolampis, Boie. -1587. Orthorhynchus, Lacép. (imaragdites, p. Boie.)-1588. Cephalepis, Loddiges. - 1589. Heliactin; Boie. - 1590. Loddigiornis, Bp. (Loddigesia, Gould. - Mulsantia, Reich.) - 1591. Spathura, Gould. (Steganura, Reich.) - 1592 . Discura, Bp.-1593. SeJasphorus, Sw. (Calliphox, Reich. ex Boie.) - 1594. Trochilus, L.

f. Mellisugeae. - 1595 . Thaumastura, Bp. (Tilmatura. Reich.) 1596. Lucifer, Less. - a. Cyanopogon, Reich. - b. Cora, Reich. - c. Elisa, Reich. - 159\%. Tryphæna, Gould. (Calliphlox, Boie.) - 1398. Callothorax, Gr. (Lucser, Reich.) - 1599. Bellatrix, Boie. - 1600. Lophornis, Less. - 1601. Gouldomyia, Bp.b. Popelairia, Reich. - 160\%. Mellisuga, $\mathrm{Br}$.

73. CYPSELIDA.

$$
\text { Stirps 18. HIANTES (Cypseli). }
$$

179. Cypseline.

Cosmop.

a. Dendrochelidoneae. - - 1603 . Dendrochelidon, Boie.

b. Cypseleae. - 1604 . Pallene, Lesson. - 1605. Achanthylis, Boie. - 1606. Cypselus, Illiger. - 1607. Tachornis, Gosse. - 1608. Panyptila, Cab.

180. Collocalinas.

1609. Collocalia, Gr.

$$
\text { Stirps 19. INSIDENTES (Nocturni). }
$$

74. STEATORNITHIDE.

181. Steatomitiman. As. 1 . Oc. is

1610 . Sleatornis, IJumboldt.

7:). (APRINULGII)E.

182. MODARGTIA. Malaiasia, Oceania.

1611. Podargus, Cuvier. - 1612. Batrachostomus, Gould. 1613. Bombycistoma, Bp. ex //ay. 
184. Nyctibine. . . . . . . . . . . America. 50

a. Nyctibieae. - 1615 . Podager, Wagl.-1616. Nyctibius, $V$.

b. Chordeileae. - 161\%. Chordeiles, $S w .-1618$. Lurocalis, Cass. - *1619. Nyctiphrynus, Bp.-1620. Antrostomus, $G$.

c. Nyetidromea: - 1621. Nyctidromus, Gould. - 1622. Sienopsis, Cassin. - 1623. Hydropsalis, Wagl. - 1624. Eleothreptus, Gr.

185. Caprinulgine.

Eur. As. Afr. Oc. 40

1625. Scotornis, Sw.-1626. Nacrodypteryx, Sw. (Seloclusa! Gr.)

- 1627. Lyncorniś, Gould. - 1628. Eurostopodus. Gould.

- *1629. Nyctiprogne, Bp. - 1630. Caprimulgus, $L$.

Ordo 4. INEPTI (Inertes).

76. DIDIDE.

186. Epyornithine . . . . . . . . Extinct. Madagascar

1531. Epyornis, Isidore Geoffr. — *1632. Ornithaptera, Bp. *1633. Cyanornis, $B p$.

187. Didine.

Extinct.

1634. Didus, L. - 1635. Pezophaps, Stricklard.

Ordo 5. COLUMBE (Gemitores).

Tribus 1. PLEIODI.

77. DIDUNCULIDE.

188. Didunculine.

1636. Didunculus, Peale (Gnathodon, Jard.-Pleiodus, Reich.)

\section{Tribus 2. GYRANTES.}

78. TRERONIDE.

189. Treronine. . . . . . . . . . . As. Afr. Ocean.

*1637. Butreron, Bonap. - 1638. Sphenæna, Bp.- 1639. Treron, Vieill. - a. Toria, Hodgs. - b. Treron, Vieill 1640. Vinago, $B p$. ex Cuvier.

190. Ptilopodine. . . . . . . . . . . . . As., Oc.

* 1641 . Leucotreron, Bp. - *1642. Ramphiculus, Bp.-1643. Pti lopus, $S w .-1644$. Kurutreron, Bp.-—*1645. Chrysæna, Bp. 191. Alectranadinz. . . . . . . Madagascar, Sechelles :

*1646. Chlamydæna, $R p .-1647$. Alectrænas, $G r$.

79. COLUMBIDE.

192. Lopirol EMiNx.

Australia

1648. Lopholæmus, Gr. 
193. Campophagina As, Ocean.

1649. Carpophaga, Selby. - 1650. Megaloprepia, Reich. *1651. Hemiphaga, Bp. - 1652. Alsecomus, Hodgs. 1653. Janthænas, Reich. - 1654. Zonænas, Reich. *1655. Leucomelæna, Bp. - 1656. Myristicivora, Reich.

19؛. Columbixe . . . . . . . . . . . Cosmop. 50

a. Columbere. - 165\%. Palumbus, Kaup. (Tæuiænas, Reich.) 1658. Dendrotreron, Hodgs - - 1659. Columba, L. (Verru'ia! Flem. - Craspedænas! Reich.) - a. Lithcenas, Reich. - b. Chlorcenas, Reich.-1660. Stictænas, Reich. - 1661. Patagiænas, Reich. - 1662. Lepidænas, Reich. - * 1663. Crossophthalmus, Bp.

b. Macropygieae. - 1664. Macropygia, $S w .-166$. Tomopeleia, Reich. - 1666. Reinwardtænas, Bp. - 166\%. Ectopistes, $S w$.

193. Turturine. . . . . . . . . Eur., As., Afr., Oc.

1668. Turtur, Br. - 1669. Peristera, Boie. - 1670. Geopeleia, Sw. - 1671. Tympanistria, Reich. - 1672. Stictopeleia, Reich. - 1673. OEna, Selby.

196. Zenaidine. . . . . . . . . . America.

1674. Chamæpelia, Sw. — *1673. Zenaidura, Bp. - 1676. Zenaida, Bp. - 1677. Columbina, Spix. - 1678. Starnænas, Bp. - 1679. Leptoptila, Sw. - 1680. Geotrygon, Gosse.1681. Oropeleia, Reich.

197. Pilapine. . . . . . . . . . . Ocean.

1682. Trugon, Hombr. et Jacq. - 1683. Phlegænas, Reich. *1684. Pampusana, Bp.-1685. Petrophassa, Gould. 1686. Phaps, Selby. - 1687. Leucosarcia, Gould. - 1688. Chalcophaps, Gould. - 1689. Ocyphas, G. - 1690. Geophaps, G.

80. CALLILNADIDE.

198. Calumenadine.

Ocean.

1691. Callixnas, Gr.

81. GOURIDE.

199. Gounisa.

1692. Goura, Flem.

Ordo 6. HERODIONES.

Tribus 1. Gitedes.

82. PHANICOPTERIDA. 
83. GRUIDE.

201. Gruine . . . . . . . . . Cosmopol.

1694. Grus, Gesn. - 1695. Antigone, Reich.-*1696. Geranus,

Bp. - 1697. Laomedontia, Reich. - 1698. Anthropoides,

Vieill. - 1699. Balearica, Br.

202. Euripygine . . . . . . . . . Amer. m.

1700. Euripyga, Ill.

203. Araminee America.

1701. Aramus, Vieill.

84. PSOPHIID瓜.

204. PSOPHINE.

Amer. m.

1702. Psophia, Barrère.

85. CARIAMIDE.

205. Cariaminte.

Amer. m.

1703. Cariama, Marcgr. (Microdactylus, Geoffr.)

86. PALAMEDEIDE.

206. Palamedelnet . . . . . . . . . Amer. m.

1704. Palamedea, L. - 1705. Chauna, Ill. - a. Chauna, Reich.

- b. Ischyornis, Reich. - Hitchcockia, Reich. Foss.

\section{Tribus 2, OICONIF.}

87. DRONADIDE.

207. Dromadine.

1706. Dromas, Paykull.

88. CICONIIDE.

208. Ciconnine. . . . . . . . . . . . Cosmopol. 15

1707. Argala, Leach. (Leptoptilos, Temm.) - 1708. Ciconia, L. -

a. Ciconia, Reich.-b. Melanopelargus, Reich.-1709. Sphenorhynchus, Ehrenb.- 1710 . Mycteria, L.-Pelarganax, Reich. Foss. - Pelargides, Reich. Foss.

209. Anastomatina

As., Afr. 2

1711. Anastomus, Bonnat. - 1712. Hiator, Reich.

89. ARDEIDE.

210. Ardeine. . . . . . . . . . . . . Cosmopol.

a. Ardeese. - *1713. Ardeomega, Bp. - 1744. Typhon, Reichenb. - 1715. Ardea, L. - 1716. Herodias, Bp. ex Boie. - 1717. Egretta, Bp.-1718. Agamia, Reich._*1719. Bubulcus, Pucheran. - 1720. Buphus, Boie. - 1721. Ardetta, Gr. - 1722. Ardeola, Bp. ex Boie.

b. Botaureas. - 1723. Botaurus, Br. - 1724. Butorides, Harll. - 1725. Tigrisoma, $S w$. - *1726. Gorsakius, $B p$. 
c. Nycticonaceae. - 172\%. Pilherodius, Reich. - 1\%98. Nyctherodius, Reich. - 1799. Nycticorax, Br.

211. Scopina.

Africa. 1

1730 . Scopus, $B r^{\circ}$.

90. CANCROMLDÁ.

212. Cancromixe.

Amer: m.

1731. Cancroma, $L$.

213. BaLmenicepiate

Afr.

1732. Balaniceps, Gould.-Prolopelargus, Reich. Foss.

91. PLATALEIDA.

214. Plataleina. - . . . - Eur., As., Afr., Amer., Oc. 8

1733. Platalea, L. - a. Platalea, Reich. - b. Spatherodia, Reich.

c. Ajaia, Reich. - d. Leucerodia, Reich.

92. TANTALIDAE.

215. Tantaline. . . . . . . . As. Afr. Am.

1734. Tantalus, L. - a. Tanlalus, Reich. - b. Tantalides, Reich.

- Tantaleus, Reich. Foss. - Talantatos, Reich. Foss.

פ16. Iвике. . . . . . . . As. Afr. Am. m.Oc.

1735. Ibis, Savign. Cuv. (Threskiornis, Wagl.) - 1736. Nipponia, Reich. - 1737. Carphibis, Reich. - 1738. Inocotis, Reich. - 1739. Phimosus, Wagl. - 1740. Geronticus, Wagl. (Comatibis, Reich.) - 1741. Theristicus, Wagl. - 1742. Lophotibis, Reich. - 1743. Molybdophanes, Reich. - 174. Bostrychia, Wagl. - 1745. Harpiprion, Wagl. - 1746. Cercibis, Wagl.

217. Eudocimist. . . . . . . . . Eur. As. Am.

1747. Eudocimus, Wagl. (Paribis, Geofr.) - a. Leucibis, Reich. b. Guara, Reich._1748.Plegadis, Kaup.(Nalcinellus, Bechst.nec Cuv.)

Ordo 7. GAVIR (Pelagici).

'Tribus 1. 'TO'THPALIII (Steganopodes).

93. PELECANIDE.

218. PELECANIX di

1749. Pelecanus, L. - a. Onocrotalus, Reich. - b. Catoptropelicunus, R. - c. Leptopelicanus, R.-1750. Cyrtopelicanus, R. 219. PHalachoconacinde.

1751. Phalacrocorax, Br. - 1752. Graculus, Aldr. - 1753. Hypoleucus, Reich. - 175\%. Haliæus, $1 l l$. 
220. Sulinæ . . . . . . . . . . . . . 7

175็. Sula, Br. - a. Plancus, Reich. ex Klein. - b. Sula, Reich.

- c. Piscatrix, Reich. - d. Dysporus, Reich. ex Ill.

94. TACHYPETIDE.

221. TACHypetine

1756. Tachypetes, 1ll. - Prolopelicanus, Reich. Foss.

95. PLOTIDE.

222. Plotinte.

1757. Plotus, $L$.

223. Helionnithine .

1758. Heliornis, Bonnat. - 1759. Podoa, Ill. - 1760. Podica, Less. - Deanea, Reich. Foss.

96. PHAETONIDA.

224. Phaetonine.

1761. Phaeton, L. - a. Phaeion, Reich.- b. Lepturus, Moehring. - c. Tropicophilus, Leach.

Tribus. 2. LONGIPENNES.

97. PROCELLARIIDE.

225. DioMEDEINE.

Cosmop.

1762. Diomedea, L. - a. Diomedea, Reich. - b. Thalassarche, Reich. - c. Phcebetria, Reich. - d. Phcebastria, Reich.

226. Procellarinne. . . . . . . . . . . Cosmop. 50

a. Puffineae. - 1763. Puffinus, Br. - 1764. Priofinus, Hombr. et J. - 1765. Thyellus, Gloger. - 1766. Ardenna, Reich. 1767. Majaqueus, Reich.

b. Procellarieae. - 1768. Bulweria, Bp. - 1769. Thalassidroma, Vig. - a. Thalassidroma, Bp. - b. Oceanodroma, Reich. 1770. Procellaria, L. - a. Oceanilis, Keis. et Blas. - b. Pelagodroma, Reich. - c. Procellaria, Bp.

c. Fulmareae.-1771. Ossifraga, Hombr. et Jacq. - 1772. Fulmarus, Leach. - 1773. Priocella, Hombr. - 1774. Daption, Stephens. - 1775. Prion, Lacép. (Pachyplila? lll.)

d. Wagellese. - 1776. Wagellus, Ray. - 1797 Rhantistes, Kaup. - 1778. Thalassoica, Reich. 
98. LARIDE.

228. Lestrigink.

Cosmop

1780. Lestris, Ill. - 1781. Coprotheres, Reich. - 1782. Stercorarius, Bp. ex Br. - 1783. Cataracta, Brunn. - Cimoliornis, Owen, Foss.

229. Larixe.

Cosmop.

a. Larcae. - *1784. Procellarus, Bp. - 1785 . Leucophæus, Bp. - "1786. Blasipus, Bonap. - *1787. Gabianus, Bp. 1788. Larus, L. - 1789. Laroides, Brehm. - 1990 . Gavina, Bp. -- *1791. Gelastes, Bp. - 1792. Pagophila, Kaup. 1793. Rissa, Brunn. - 1794. Rhodostetia, Macgill.

b. Xemeae. - * 1795. Adelarus, Bp. - 1796. Ichthiætus, Kaup. — *1797. Atricilla, Bp. - 1798. Gavia, Br.- 1799. Chroicocephalus, Eyton.- $* 1800$. Creagrus, Bp.-1801. Xema, Leach.

230. Sternixas. . . . . . . . . . . Cosmop.

a. Sternere. - 1802. Phaetusa, Wagl. - 1803. Pelecanopus, Wagl. - 1804. Onychoprion, Wagl.-1805. Haliplana, Wagl. 1806. Hydroprogne, Kaup. - 1807. Thalasseus, Boie. 1808. Gelochelidon, Brehm.-1809. Sterna, L. - 1810. Seena, Blyth. - 1811. Sternula, Boie. - 1812. Gygis, Wagl. 1813. Hydrochelidon, Boie.

b. Anoca. 1814. Anous, Leach. - b. Megalopterus, Boie. 1815. Larosterna, Bly $h$. -1816 . Procelsterna, Lafr.

231. Ruxnchopina. Maria intertr.

1817. Rhynchops, $L$.

99. CHIONIDE.

232. Chioxinat.

Amer. m.

1818. Chionis, Ill.

Tribus 3. URnNaTOates (Brachypteri).

100. ALCID E.

233. $\Lambda_{\text {LCIN }}$.

Terræ arct.

6

1819. Alca, L. - 1820. Utamania, Leach.

234. Pinlemidina.

As. S. Am. s. 14

1821. Normon, Ill. (Fratercula, Br.) - a. Lunda, Pall. - b. Gymnoblepharum, Brandt. - c. Ceraloblepharum, Brandt. 1822. Sagmatorhina, Bp. - 1823. Ceratorhyncha, Bp. (Chimerini, Eschsch.)- 1824. Ciccronia, Reich. - 1825. Ombria, Eschscholtz. - 1826. Phaleris, Temm.- a. P'haleris, Brandt.b. Tyloramphus, Brandt. - 1827. Plychoramphus, Brandt. 
233. Uninim.

Terræ aret.

1828. Uria, Br. - a. Lomvic, Brandt. - b. Cepphus, Pall. (Grylle, Brandt ) - 1829. Apobapton, Brandl. - a. Brachyramphus, Brandt. - b. Synthliboramphus, Brandt. - 1\$30. Mergulus, Vicill. (Cephus, Chei.)

101. COLYMBIDE.

236. Colymbin.e.

Terræ arctic. 4

1831. Colymbus, L. (Eudytes, $I t t$.)

102. PODICIPIDE.

237. Podicipine. . . . . . . . . . Cosmopolit.

1832. Podiceps, Lath. (Colymbus, Br.)- - . Podiceps, Kaup. (Colvmbas, Reirh.) - b. Pedaithyia, Kaup. - c. Dyles, Kaup. - d. Otodytes, Reich. - e. Tachybaplus, Beich .-- f. Dasyptilus, Sw. - 1833. Sylheocyclus, Bp. (Podilymbus, Less. - Podiceps, Reich)

Ordo 8. PTILOPTERI. (Impernes.)

103. SPIIENISCIDR.

238. Spheniscinte.

Terra antarct.

1834. Aptenodytes, Forster. - 1835. Spheniscus, Br. 1836. Eudyptes, Vieill. - 1837. Catarhactes, Br. - 1838. Pygoscelys, Wagl. - 1839. Dasyramphus, IIombron et Jacq.

Subclassis 2. PRECOCES. (Grallatores.)

\section{Ordo 9. GallLiñ. (Rasores.)}

104. MESITIDE.

\section{Tribus 1. SEERACER.}

239. Mesitink.

Madagascar.

184.0. Mesites, Geoffr.

403. MEGAPODIIDE.

240. MEgaPODIINE.

Ocean. 8

18\$1. Megapodius, Quoy el Gaimard.

24. Talegalinas.

Occan.

1842. Leipoa, Gould. -- 1843. Talegalla, Sw. (Cubcurue, Su.) 1844. Megacephalon, Temm.

106. ROLLULIDA.

212. Rollulinte. Malaias.

1845. Rollulus, Vieill. - 1816. Cryptonyx, IIl.

107. NUMIDID $E$.

243. Agelastina .

1847. Agolastes, Temm.

Extr. des Axnales des sgences naterelees. 
944. Numidne:

Africa.

1848. Numida, L. - a. Numida, Reich. - b. Querelea, Reich.

- 1849. Guttera, Wagl. - 1850. Acryllium, Gr.

Tribus 2. GALLINACEA.

Cohors 1. CRACES. (Longicaudce americance.)

108. MELEAGRID E.

245. Meleagrine

Amer. s. 2

1851. Meleagris, $L$.

109. CRACIDA.

246. Cracine.

Amer. calid.

1852. Crax, Barr. - 1853. Pauxi, Temm. - 1854. Urax, Cuv.

110. PENELOPIDE.

247. Penelopine. • . . . . . . . . . Amer. calid.

1855. Salpiza, Wagl. - 1856. Penelope, Merr. - 1857. Abur -

ria, Reich. (Chamxpetes? Wagl.) -1858 . Penelops, Reich. 1859. Ortalida, Merr.

248. Oreopuasinks.

Am. centr.

1860. Oreophasis, Gr.

Cohors 2. GALLI. (Longicaudice asiaticae.)

111. PAVONIDE.

249. Argine.

1861. Argus, Temm.

250. Pavonine . . . . . . . . As. m. Occan.

a. Pavoneac. - 1862. Pavo, L. - a. Pavo. - b. Spicifer.

b. Polyplectroneae. - 1863. Polyprectron, Temm. - a. Emphania, Reich.-b. Polyplectron, Reich.-㫧 864 . Chalcurus, Bp.

112. PIIASIANIDE.

231. Pinasianify . . . . . . . . . As. Oc. Eur. 25

a. Satyrease. - 186 מ. Satyra, $L$. (Tragopan, Cuv.) - 1866. Pucrasia, $G r$.

b. Phassianeace - 1867. Thaumalea, Wagl. - 1868. Phasianus, L. - 1869. Graphephasianus, Reich. - 1870. Syrmaticus, Wayl. - 1871. Nycthemerus, Sw. - 1872. Lophophasianus, Reich. - 1873. Euplocomus, Temm. (Gennxus, Wagl.)

c. Galleace. - 187\%. Gallus, $L$.

252. Lophopiorints. As. m. Oc. 10

187\%. Lophophorus, Temm. - 1876. Alectrophasis, $G r$. (Gallophasis, Itorlgs.) - 1877. Crossoptilon, Hodgs. - 1878. Acomus, Reich. - 1879. Grammatoptilus, Reich. 
Cohors 3. PERDICES. (Brevicaudce.)

113. THINOCORIDE.

253. Thinocorine. . . . . . . . . . . . . Am. m. 7

1880. Attagis, Is. Geoffr. - 1881. Thinocorus, Eschsch.

114. PTEROCLIDE.

25\%. Pteroclinte. . . . . . . . . . . Eur. As. Afr. 16

1882. Pterocles, Temm. (Enas, Vieill.) - *1883. Pteroclurus, $B p$.

- 1884. Psammænas, Blylh.

255. Syrrhaptine

As. centr.

1885. Syrrhaptes, Ill. (Heteroclitus, vieill.)

115. TETRAONINÆ.

256. Tetraonine.

Eur. As. Am. s.

1886. Tetrao, L. - 1887. Lyrurus, $S w$. - 1888. Centrocercus,

Sw. - 1889. Canace, Reich. - 1890. Cupidonia, Reich. 1891. Bonasia, Bp. - 1892. Lagopus, Br.-a. Oreias, Kaup.

- b. Allagen, Kaup. - c. Lagopus, Kaup.

116. PERDICIDAE.

257. Perdicine.

a. Tetraogalleae. - 1893. Tetraogallus, J.Gr. - 1894. Lerwa, Hodgs. - 1895. Galloperdix, Blyth. - 1896. Plectrophora, Gr. - 1897. Ithaginis, Wagl.

b. Francolinese.-1898. Francolinus, Gesn.-1 899. Ortygornis, Reich. - 1900. Didymacis, Reich. - 1901. Pternistes, Wagl.

c. Perdiceac. - 1902. Caccabis, Kaup. - 1903. Perdix, L. 1904. Ammoperdix, Gould.

d. Starneac. - 1905: Starna, Bp. - 1906. Margaroperdix, Reich. - 1907. Ptilopachys, Sw. - 1908. Hepburnia, Reich. - 1909. Arboricola, Hodgs.

258. Ortygine

Americ.

1910. Odontophorus, Vieill. - 1911. Dendrortyx, Gould. 1912. Ortyx, Steph. - 1913. Cyrtonyx, Gould. - 1914. Eupsychortyx, Gould. - 1915. Callipepla, Wagl. - 1916. Lophortyx, $B p$.

259. Cotunnicine . . . . . . . . Eur. As. Afr. Oc. 28

1917. Perdicula, Hodgs. - 1918. Synoicus, Gould.-*1919. Ortygion, Bp. ex Keys. - 1920. Coturnix, Gesner.

260. Tunnicina. . . . . . . . . Eur. As. Afr. Oc. 30

1921. Turnix, Bonnat. - 1922. Ortygis, Ill. - 1923. Oxyteles,

Vieill. - 1921. Pedionomus, Gould. 
I17. CRYPTURMDE.

261 Cripturixa.

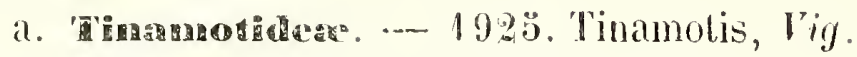

b. Croprouace. - 1926. Hinamus. Lath. - 192\%. Crypturus, Ill. - 1928. Nohnura, Hagl. - 1929. Rhynchoúis. Spix.

962. Eunromuxis.

1930. Ludromia, Is. Geo/fir.

Grib 10. GRALLE.

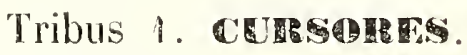

1180 TIDE

263. Othiке . . . . . . Eur. As, Afr. Occan。

1931. Otis, L. - 193\%. Houbara, Bp. (Chlamydotis, Less.) 1933. Tracholotis, keichcmbach. - 1934. Tetrax, Bonaparle. _* 1935. Afrotis, Bp. - 1936. Lophotis, Reich.-1937. Sypheotis, Less. (Comatolis, Reich.) - 1938. Lissotis, Reich. -. 1939. Eupodolis, Less._- 1940 . Choriotis, Bp.

119. CHARADRIDDAE.

264. Hotcremiñ. • . . . . . . . . . Cosmopol. 8

1941. Burhinus, Illig. - 1942. Edicnemus, Eelon - 1943. Esacus, Less. - 1914. Carvanaca, IIodgs.

260̈. Chamadrinz. • . . . . . . . . . . Cosmopol.

a. Charmadrouere. - 1945. Pluvialis, Br. - 1946. Squatarola, Cuv.- 1987. Morincllus, Ray. (Eurlomias, Buie.)- 1948. Oreophilus, Jard. - 1949. Oxyechus, Reich. - 1950. Charadrius, L. - 19:31. Egiahus, Reich. - 195\%. Ochthodromus, Reich. - 1933. Thinornis, G\%. - 195\%. Pipis, Licht.

b. Vandelleac. - 1955. Vandlus, Br. - 1956. Chottusia. Bp. - 1937. Zonibyx, Reich. - 1938. Erylhrogonys, Goule. 1959. Moplopterus, Bip. -- a. Stephanilyx, Reich. - b. Bicionopterus, Reich. - c. Iloploplerus, Reich. - d. Sarcogrammus, Ricich. - 1960 Sarciophoras Strichl.-1961. Lobivanellus, Strickl. - a. Tylibyx, Reich. - b.' Tiphidiopierus, Reich. c. Lobivanellus, Gould. - 1962. Pluvianus, Vicill.

266. Cunsondix . . . . . . . Eur. As. Afr. Oc.

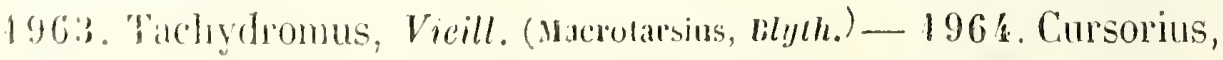
Lath.-- $196 \%$. Chalcopterus, Rech.

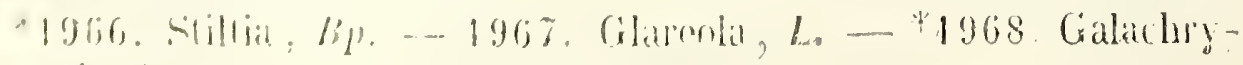
iil lip. 
121. ILEMATOPODIDE.

268. Strepsilin.e.

1969. Pluvianellus, Ifombr. et $J$. 1970. Aphriza, Aud. 1971. Strepsilas, $L$.

269. H.enatopodin.e.

1972. Hæmatopus, L. - a. Hcomalopus, Reich. - b. Ostralegus, Reich. - c. Melanibyx, Reich. - Argoides, Hithcock, Foss.

\section{RECURVIROSTRIDE.}

270. Recurvirostrin.e

Cosmop.

a. Himantopodere. - 1973. Himantopus, $\mathrm{Br}$.

b. Recurvirostreae. - 1974. Cladorhynchus, Gray. (septoriynclins, Dubus.) - 1978. Recurvirostra, $L$.

123. PHALAROPODIDE.

271. Pinalaropodine.

Arct.

1976. Phalaropus, Br. - 1977. Lobipes, Cuv. - 1978. Holopodius, $B p$.

\section{SCOLOPACIDA.}

272. Prosobonin.e.

1979. Prosobonia, $\mathrm{Rp}$.

273. Scolopacixe.

Cosmop.

1980. Rhynchæa, Cuv. - 1981. Scolopax, L. - 1982. liusticola, Bp. (Plituheli, vieill.) - 1983. Xylocota, Bp. - 1984. Gallinago, Bp. - a. Pelorychus, Kaup. - b. Gallinago, Ray. c. Limnocryples, Kaup. - 1983. Macroramphus, Leach.

274. Tringines

Cosmop.

a. Tringeae. - 1986. Eurynorhyncus, Nills. - 1987. Heteropoda, Bp. - 1988. Hemipalama, Bp. - 1989. Calidris, Ill. 1990. Pelidna, Cuv. (Erolia! vieill. - Falcinellus! Cuv.)-1991. Limicola, Koch. - 1992. Tringa, L. - 1993. Machetes, Cuv. (Philomachus, Moehr. - Pavoncella! Leach.) - 1994. Phegornis, Gr.

b. Totanneac. - 1995. Actitis, Ill. (Tringoiles, Gr.) - 1996. Actiturus, Ill. (Bartramia, Less. - Eu'iga, Nutt.) - 1997. Totanus, Bechst. - a. Ilyornis, Kaup. — b. Gambetta, Kaup. - c. Erythroscelus, Kaup. - d. Rhyocophilus, Kaup. - e. Helodromus, Kaup. - 1998. Catoptrophorus, Bp. (Symphemia, Rafin. - Iorli1es, Kaup.) - 1999. Glottis, Nilss. (Limicula, Leach.)

c. Cinnosere. - 2000. Anarhynchus, Quoy ol Gaim.-2001. Te-

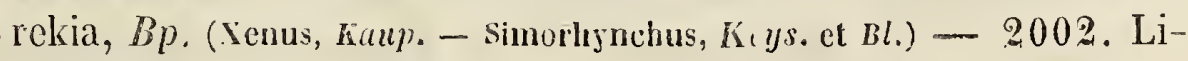
mosa, Br. ( Fedoa, steph.) - 2003. Numenius, Mochr. (Phorolus, Cul.) - 2004. Ibidorhyncha, rig. 


\section{Tribus 2. ALECTORIDES.}

120ั. PARRIDE.

275. Parrine

As. Afr. Am. Oc.

2005. Parra, L. - 2006. Metopidius, Wagl. - 2007. Hydrophasianus, Wagl. - 2008. Hydralector, Wagl.

426. RALLIDA.

276. RaLLind.

Cosmopol. 100

a. Balleac. 2009. Aramides, Pucheran. (Rallina, Reiehenbach.) 2010. Biensis, Pucher. - 2011. Rallus, L. - 2012. Hypotænidia, Reich. - a. Hypotonidia, Reich. - b. Euryzona, Reich. c. Lewinia, Reich. - *d. Lalerallus, Bp. - 2013. Hypnodes, Reich. - 2014. Porzana, Vieill. - 2015. Ortygometra, Barrère. - 2016. Zapornia, Leach. (Phalaridion, Kaup.) *2017. Coturnicops, Bp. - 2018. Crex, Bechst. - 2019. Corethrura, Reich.

b. Gallimaleae. - 2020. Notornis, Owen. - 2021. Porphyrio . Br. - a. Porphyrio, Reich. - b. Casarornis, Reich. 2022. Porphyrula, Blyth. (Jonornis, Reich.) - 2023. Tribonyx, Dubus. - 2024. Gallinula, Br. — 2025 . Gallinulopha, $B p$. 2026. Amaurornis, Reich. - *2027. Porphyriops, Puch. 2028. Erythra, Reich. - 2029. Hydrocicca, Caban. 2030. Glaucestes, Reich.

c. Fuliceae. - 2031. Fulica, L. - a. Fulica, Reich. - b. Phalaria, Reich. - b. Lysca, Reich. - 2032. Lupha, Reich.*2033. Licornis, $B p$.

277. OcroROMINE.

Afr. Occan.

2(13. Ocydromus, Strickl. (Gallirallus, Lafr.) - 2035. Eulabeornis,

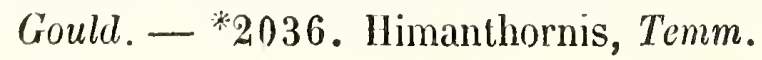

Ordo 11. ANSERES. (Natatores.)

127. ANATIDT.

278. Crgniaf Cosmop.

a. Cygneac. - 2037. Olor, Wagl. - 2038. Cygnus, L. 2039. Chenopsis, Wagl. - 20\%0. Coscoroba, Bp.

279. ANSERINE

Cosmop.

b Ansereac. - 20\&1. Cygnopsis, Brandt. 2042. Anser, Barvere. - a. Anser, Reich. - b. Marilochen, Reichenbach. 20 43. Chen, Brehm. (Chionochen, Reich.)... 2044. Eulabeia, Reich. - 2015. Nellapus, Brandl. 
c. Bernicleae. - 2046. Bernicla, Aldr. - a. Bernicla, Reich. - b. Leucopareia, Reich._2047. Tænidiestes, Reichenbach. 2048. Chlamidochen, Bp.

d. Cereopseae. - 2049. Cereopsis, Temm.

e. Plectroptereae. - 2050. Sarkidiornis, Eylon. - 2051. Chenalopex, Steph. - 2052. Plectropterus, Leach. - 2053. Anseranas, Less.

280. Anatine . . . . . . . . . . . Cosmop.

f. Tadorneae. - 2054. Casarca, Bp. - 2055. Radjah, Reich. 2056. Tadorna, Leach. - Todarna, Reich. Foss. - 2057. Dendrocygna, $\boldsymbol{S} w$. (Leptotarsis, Ey .) $^{\circ}$

g. Anatese.-2058. Cairina, Flem.-2059. Anas, L. -2060. Rhodonessa, Reich. - 2061. Chaulelasmus, Gr. - 2062. Malacorhynchos, Sw. - 2063. Spatula, Boie. (Rhynchaspis, Leach.) - 2064. Plerocyanea, Bp. - 2065. Querquedula, Steph. - 2066. Aix, Sw. (Cosmonessa, Kaup.) - 2067. Mareca, Steph. - 2068. Stictonetta, Reich. - 2069. Marmaronetta, Reich. - 2070. Dafila, Flem. - 2071. Pæcilonetla, Eyton.

281. Fuliguline. . . . . . . . . . . . Cosmop.

h. Erismatureae. - 2072. Biziura, Leach. - 2073. Thalassornis, Eyton. - 2074 . Erismatura, Bp. (Undina, Gould.) 2075. Nesonetta, Gr.

i. Clanguleae. - 2076. Micropterus, Less. - 2077. Harelda, Leach. - 2078. Clangula. Flem - 2079. Camptolæmus, Gr. (Camptorlynchus, Eyton.)

k. Somaterieae. - 2080. Somateria, Leach. - 2081. Stelleria, $B p$. (Eviconetta, Gr.) - 2082. Lampronetta, Brandt.

1. Didemiere. - 2) 83. Polionetta, Kaup. - 2084. Melanetta, Brehm. - 2085. Oidemia, Flemm.

m. Fuliguleae. - 2086. Fuligula, Ray. (Fulix, Sund. ex Cicerone.) -2087. Marila, Reich. - 2088. Nyroca, Flemm. - b. Ethyia, Boie. - 2089. Branta, Boie. - 2090. Hymenolæmus. Gr. (Malacorhynchus, $p$. Wagl.)

128. MERGID瓜.

282. Mergiza.

2091. Merganser, Br. - 2092. Mergus, L. - 2093. Lophodytes, Reich. - 2094. Mergellus, Selby.

283. Merganettine. • . . . . . . . . . . Austr. 3

2095. Merganetta, Gould. 
Ordo 12. STRUTIIONES. (Rudipennes.)

129. STRUTHIONID E.

28. Struthonisa.

Africa

2906. Struthio, $L$.

จ8̈. RIIEIx.

Am m. Or.

$\dddot{3}$

209\%. Rhea, Mohring. - a. Bellowa. Reich. Foss. - b. Berceyn-

thia, Reich. Foss. - Cybele, Reich.Foss.-- 2098. Dromaius,

Vicill. - 2099. Casuarius, Frisch.

\section{DINORNITHIDA:}

286. Dinornituina.

N. Zeland. Foss.

Dinomis, Owen. Fuss. - Moa, Reich. Russ. - Movia, Reich

287. Enenn. . . . . . . . . . . N. Zeland Foss

Emeus, Reich. Foss. - Cela, Reich. Foss. - Syomis, Reich.

298. Palapterigixis.

Palapteryx, Owen. - Aptornis, Mantell. Foss. - Ansmaiopteryx, Roich. Foss.

\section{APTERYGIDR.}

289. ArtengGinis.

2100. Apleryx, Shave.

Nova-Zelandia.

Specierum Avium numerus.

8300

\section{ADDENDA.}

2101. $\left.133^{n}\right)$ Psittovius, bp. - 2102. (22) Graydidascalus, Bp. - 2103. (12) Prosopeia, Bp.--2104. (70) Glossopsitta, Bp.--2105. (71a) Psitteuteles, Bp. - 2106. (212i) Smilhiglaux, Bp.-2107. (255i) Cissa, Bp.-2108. 308a) Hyobletes, Glog. - 2109. (510a) Hodoiporus Bp. cx Reich. - 2110. (385a) Atrichia, Gould. - 2111. (386 $)$ Pycnoptilus, Gould, 1850 - 2112. (881\%). Meristes, Ip . ex Reich. - 2113. (706a) Polystictus, Reich. - 2144. (1000a) Xocephus, lip. - 2115 (1019) Negabias, Verb. - 2116. (1015a) Diopho-

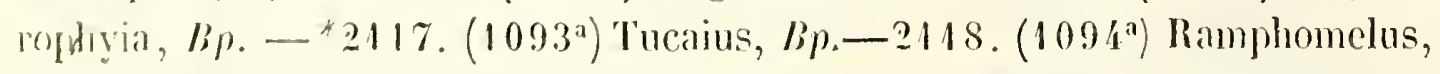
lip. - 2119(109:3") Piperivorus, Bp.-2190. (1096") Ramphoxanthus, bp. 
Extrait de la Revue et Magasin De Zoologia.

$\mathbf{N}^{0} \mathbf{8},-1854$.

\section{TABLEAU}

DES

\section{OISEAUX DE PROIE}

Par S. A. le Prince C.-L. Bonaparte.

\section{CONSPECTUS ACCIPITRUM}

AVIUM Ordo II. ACCIPITRES. (Rapaces.)

Familia 5. VULTURID E.

Subfam. 16. Cathartine.

1. Saricoramphus, Duméril. (Gypagus, Vieill.) - 1. papa, L.

2. Gryphus, Is. Geoffr. - 2. cuntur, Dum. (condor, Less.) - 3. californianus, Shaw.

3. Cathantes, Ill. (Cahharista, Vieill.) - 4. aura, L. (septentrionalis, Wied.) - 5. jota, Molina. (burrovianus? Cass.)

4. Conagres, Geoffr. - 6. atratus, Wils. (urubu, Vieill.) - 7. brasiliensis, $\boldsymbol{B r}$. (foetens? Ill.)

Subfam. 17. Vulturina.

5. Gyps, Savign. - 8. fulvus, Gm. (vulgaris, Sav.) 9. occidentalis, Schlegel. - 10. ruppelli, Bp.- 
11. Kolbi, Daud. - 12. indicus, Scopoli. - 15. bengalensis, Lath. tenniceps, Hodgs.)

i. Vubture $\mathbb{L}$.

*a. Lophogyps, Bp. - 14. oceipitalis, Burchell. (eulophur: elirenb.)

b. Vultur, Bp. - 15. monachus, L. (cinerens, Gm.)

7. Orogrs, Gr. - 16. auricularis, Dand. - 17. nubicus, Smith (imperialis, Temm.) - 18. calvus, Scopoli. (ponticerianus, Lath.)

8. Neophon, Sav. - 19. phieatus, Murchell. (carunculaus, Smith.) - 20. percnopterus, $\mathbb{L}$.

Familia 6. GYPAETIUA.

Sublam. 18. Grpaetine.

9. Grpatus, Storr. - 21. barbatus, L. - 22. oceidentalis, Schleg. - 25. nudipes, Brehm.

\section{Familia 7. GYPOIIERACIDA.}

Subfam. 19. Gyponeracine.

10. Grponemax, Rump). - 2年. angolensis, Gm. (hypolurcus, Rennett.)

\section{Familia 8. FALGONID}

Sublia:n. 20. Agumank.

$$
\text { a. Aquilea. }
$$

11. Nouma. Brr.

a. Alpuilu, Kaup. - 25. fulva, L. - 26. chrysactos, L. - 27. heliaca, Savign. (imperialis, Temm.) - 28. navioides, Cuv. (rapax, Temm.) - 29. planga, Vieill. 30). llixia, Gm. 
b. Uraetus, Kaup. - 51. audax, Lath. (fincosa, Cuv. cuneicaudati, Brehm.)

c. Pteraetus, Kaup. - 52. vulturina, Daud. (nigra. Jameson. - verreauxi, Less.)

12. Pseudaerus, Hodgs. (Eutulmaelus, Blyth.) - 5j. bonelli, Temm. (ducalis, Licht. - intermedia, Boirard.) 54. grandis, Hodys. (niveus, Jerd.)

15. Onychaetus, Kculp. (Heteropus, Hodgs, nec Fit:ing.) 55. malayensis, Reinwardt. (ovivora, Jerdon.)

14. Helotarsus, Smith. - 36. ecaudatus, Daul. (iypus, Smith.!

\section{b. Halieter.}

15. Haliaetus, Sav.

a. Haliaetus, Kaup. - 37. albicilla, L. - 38. leucocephalus, $\boldsymbol{L}$.

b. Thalassaetus, Kaup. - 79 . pelagicus, Pall. (imperator, Kittlitz. - leucopterus, Temm )

16. Сuncums, Hodgs. (pontaetus, Kaup.) - 40. macei, Temm. - 41. vociferoides, O. des Murs. - 42. vocifer, Daud. - 43. leucogaster, Gould.

17. Haliastur, Selby. - 44. ponticerianus, Gm. (indus, Bodd.) - 45. leucosternon, Gould. - 46. sphenurus, Vieill. (canorus, Vig.)

18. Geranoaetus, Kaup, 1844. 'Heleraelus, Kaup, 1840.) 47. aguia, Temm. (melinoleucus et fuscescens, Vieill.)

\section{c. Pandionece.}

19. Pandion, Sav. - 48. haliaetus, L. - 49. albicollis, Brehm. - 50. carolinensis, Gm. - 51. leucocephalus, Gould. (gouldi, Kaup.)

20. Poliaetus, Kaup. - 52. ichthiactus, Horsf. 53. humilis, Temm. 
21. Circaetcs, Vieill. - 54. gallicus, Gm. - 55. thoracicus, Cur. (fasciolatus, Gr. jun. - cinerens, Vieill.)

22. Sphorns, Gr. - 56. bacha, Daud. - 57. cheela, Dand. - 58. holospilus, Vig.

20). Herpetothenes, Vieill. - 59. cachinnans, $L$.

Sublam. 21. Buteonine.

\section{e. Buteonere.}

24. Ancinuteo, Brehm. - 60. lagopus, Brunn.61. sancli-johannis, Gm. - 62. strophiatus, Blyth. -- 65. hemiptilopus, Blyth.

25. Buteo, Cin.

a. Buteo, Kaup. - 64. cinereus, Gm. (vulgaris, Bechst. - mutans et fasciatus, Vieill. - pojana, Sari.) - 65. japonicus, Schley. - 66. tachardus, Dand. - 67. cirtensis, Levaill. jun. - 68. rufinus, Rupp. - 69. canescens, Hodgs. - 70. jackal, Duud. - 71. augur, Dand. - 72. plumipes, Hodgs.

b. Pocilopternis, Kaup. - 75. borealis, Gm. (harlani, Aud. var.) - 74. swainsoni, Bp. - 75. linealus, Gm. (hyemalis, Gm.) - 76. wilsoni, Bjp. (pensylvanicus, Wils. - latissinus, Ord. - platypterus, Vicill.) - 77. leucops, (ir). (infilatus, $\boldsymbol{K}$ aup.)

26. Tachytmonchis, Kamp. - 78. erythronotus, King. (allinotalus, Gr. - tricolor, Orb. - polysomus, Quoy.) 79. plerocles, Temm.

27. Butrogalus, Less. - 80. buson, Dand. (equinoctialis? Luth.) 
28. Iснтнуововus, Katup: (Busarellus, Lafr.) -81 , busarellus, Daud. (nigricollis, Vieill. - melanobroncluus, Shaw.) 29. Polionnis, Schlegel.

a. Poliornis, Kaup. - 82. poliogenys, Temm. (indicus? Gm.) - 83. liventer, Temm. (pallidus, Less.) 84. teesi, Franklin. - 85. pyrrhogenys, Schlegel.

b. Pernopsis, Dubus. - 86. rufipennis, Sundev. (erythropterus, Dubus.)

30. Kaupifalco, Bp. - 87. monogrammicus, Temm.

\section{f. Asturinea.}

71. Asturina, Vieill. - 88. magnirostris, Gm. (F. inseclivorus, Spix, Av. Br. t.8.a.) - ${ }^{\star} 89$. pucherani, Verr. (magnirostris, Mus. Paris.) - 90. nitila, Lath. 91. leucorrhoa, Quoy. - 92. brachyura, Vieill. (albifrons, Kaup.)

32. Leucopterns, Kaup. - 93. ghiesbreghti, Dubus. 94. pœcilonotus, Cuv. - 95. polionotus, $G r$. 96. lacernulatus, Temm. - 97. albicollis, Lath. 98. melanops, Lath. - 99. kuhli, Bp.

Subfam. 22. MiLvine.

\section{g. Milvere.}

33. Miuvus, Briss. - 100. regalis, Br. - 101. govinda, Sykes. - 102. affinis, Gould. - 103. niger, Br. - 104. ægyptius. Forsk. - 105. parasiticus, Lath.

34. Lophorctinia, Kaup. - 106. isura, Gould. \{pacifica? Lath)

35. Grpoictinia, Kanp. - 107 melanosterua, Gould. 


\section{h. Pernece.}

36 . Pennis, Cuv. - 108. apivora, L. - 109. madagascariensis, Smil! - 110. cristata, Cuv.

37. BAzs, Hodgs. (Lophastur, Less.) - 111. indicus, Less. 58. Avicida, Sw. - 112. reinwardti, Schlegel. 115? subcristatus, Gould. - 114. magnirostris, hanp. - 115. cuculoides, Sw. (piscator? Gm. - verreauxi, $\mathbb{L} a / r$.

39. Machemamphus, Schlegel. - 116. alcinus, Schlegel. 40. Rostrhasus, Less. - 117. hamatus, Ill. (tæuiurus? Caban.)

41. Odonthriorchis, haup). (Leptodon, Sundev., 1853, nec Web.) - 118. cayanensis, Gm. (cyanopus, Vicill. - palliatus. wied.)

42. Pegerhinus, Kaup. - 119. uncinatus, Ill. 120. wilsoni, Cass. - 121. megarhynchus, Janp.

\section{i. Elanew.}

19. Nadchenus, Vig. - 122. furcalus, $\mathbb{L}$. (carolinensis, $B r$.) 44. Comlictinis, Less. (Chelidopteryx, $\boldsymbol{K}$ p.) - 125. riocouri, Vieill.

45. Elanus, Savign. - 124. melanopteras, Daud. 125? minor, Bonat. - 128. axillaris, Lath. 127. scriplus, Gould. - 128. leucurus, Vieill.

46. Gampsonyx, Sw. - 129. swainsoni, Vig. (torquantus, Cuv. - rufifrons, Wierl. - holmi, Sundev.)

17. ICTnn, Vieill. (Nertus, Boic.)

a. Ictimia, Kaup. - 150. mississipensis, Wils.

1. Pocilopteryx, Kaup. - 131. plumbea, Vieill. 
Subfam. 23. Falconine.

j. Falconex.

48. Jeinacida, Gould. - 132. novæ-zelandiæ, Gm. 153. berigora, Vig. - 154? occidentalis. Gould. 49. Falco, Br. - 155. communis, L. - 136. anatum, $B$ p. - 157. melanogenys, Gould. - 158. peregrinator, Sundev. - 159. peregrinoides, Temm. - 140. minor, Schleg. — * 141. radama, Verr.

50. Hieropalco, Cuv. - 142. candicans, Gm. 145. islandicus, Brumn. - 144. gyrfalco, Schlegel. - 145. subniger, Gr. - 146. hypoleucas, Gould. 147. mexicanus, Licht.

51. Gennaja, Kamp. - 148. sacer, Belon. - 149. jugger, Gm. - 150. lanarius, Schlegel. - 151. barbarus, L. (alphanet, Schleg. - puniceus? Levaill. jun.) 152. cervicalis, Licht. - 15̃. tanypterus, Licht.

*52. Cinlouera, Bp. - 154. typus, Bp. - 155. rulicollis, $S w$

5е. Hypotriorciss, Buie. - 156. eleonoræ, Géné. 157. concolor, Temm. - 158. severus, Horsf 159. frontatus, Gould. - 160. subbuteo, L. 161. deirolencus, Temm. - 162. aurantius. Gm. - ${ }^{\star} 165$. auranticulus, Bp.

54. Esalon, Gr. - 164. lithofalco, Gm. - 165. fomoralis, Temm. - 166. ardesiacus, Vieill. - 167. columbarius, $\boldsymbol{L}$.

k. Tinnunculea.

35. Tinnunculus, Vieill.

a. Tinnunculus, Kaup. - 168. alaudarius, Briss. 169. japonicus, Schl - 170. guttatus, Brehm. - 
171. moluccensis, Schleg. - 172. rupicolus, Dand. - 175. rupicoloides, Smilh. - 174. cenchroides. Vig. - 175? paradoxus, Brehm. - 176. rupicoleformis, Wurtemberg. - 177? intermedius, Brehm. - 178? fascialus, Brehm.

b. Tichornis, Kaup. - 179. cenchris. Naum. (tinnunculoides, Temm.) - 180. punctalus, Cuv.

c. Pocilornis, Kaup. - 181. sparverius, L. 182. sparverioides, Vig. - 185. cinnamomeus, $S w$. - 184. isabellinus, $S w$. - 185. gracilis, $S w .-$ * 186 ? fraseri, $B P$.

56. Erixhnopus, Brehm. - 187. vespertinus, L. (rufipes, Beseke. - desertorum, Daud. ex Levaill. Afr.t. 17, jun.)

57. Polomerax, Kanp. - 188. semitorquatus, Smith.

58. Jerax, Vig. - 189. coerulescens, L. - 190. bengalensis, Briss. - 191. sericeus, Kittl.

\section{Harpagea.}

59. Malpagus, Vig. - 192. bidentatus, Lath.-. 193. diodon, Termm.

60. Splzhptens, halu) - 194. circumscriptus, Iraup.

Subfam. 24. Accipitrine.

in. Spizalece.

(i1. Lopharers, K(uly). - 195. occipitalis, Daud.196. Licneri, Gerv. - 197. isidori, O. des Murs.

62. Ptehinda, fraup. - 198. tyrannus, Wied. 65̃. Sirateres, Vieill. - 199. bellicosus, Dand. 2009. coronalus, L. - 201. spilogaster, Dubus. (zonuris, de Mull.1.1, jun.) - 202. ornatus, Dand. 
64. Jeraetus, Kaup. - 203. morphnoides, Gould. 204. pennalus, Gm. - 205. minulus, Brehm.

65. Limnaetus, Viy. - 206. cirrhatus, Gmelin. 207. orientalis, Temm. - 208. lanceolatus, Temm. 66. Spizastur, Less. - 209. melanoleucus, Vieill.

n. Morphnea.

67. Thrasaetus, Gr. - 210. harpyia, L. (destructor, Daud.)

68. Harpyhalietus, Lafr. - 211. coronatus, Vieill. nec $L$. (azaræ, Kaup.)

69. Morphnus, Cuv. - 212. guianensis, Daud. (somnini, Shaw.)

70. Hypomorphus, Cab. - 213. meridionalis, Lath. (rutilans, Licht.)

71. Unubitinga, Less. - 214. longipes, Ill. (Aq. brasiliensis, $\mathrm{Br}$ ) - 215. solitarius, Tschud. (mexicanus, Dubus. - anthracinus, Licht)

72. Craxirex, Gould. - 216. unicinctus, Temm. 217. gallapagoensis, Gould.

73. Dedalion, Bp. (Dædalia!) - 218. longicaudus, Less.

o. Accipitrew.

¿ Astures.

74. Rhynchonegus, Вp. - 219. brachypterus, Temm. - 220. dynastes, Verr.

75. Micrastur, Gr. p.- 221. xanthothorax, Temm. 222. concentricus, Illig. - 225. guerilla, Cassin.

76. Lophospizia, Kaup. - 224. trivirgata, Temm.

77. Leucospizis, Kaup. - 225. novæ-hollandiæ, Gm. 
78. Astur, Bechst. - 226. radialus, Lulh. - 227. melanoleucus, Smilh. - 228. pálumbarius, $\boldsymbol{L}$. 229. alricapillas, Wilson. (regalis, Temm.)

79. Cooperastur, Bp. - 230. pectoralis, Cuv. 251. stanleyi, Audub. (cooperi, Bp.) - 232. pileatus, Wied. - 255? bicolor, Vieill. (variatus, Cuv.) 20́4. poliogaster, Temm.

ب Accipitres.

80. Geranosprza, Taup. - 255. gracilis, Termm.

81. Melierax, Gr. - 256. musicus, Dand. - 237. poIyzonus, Rupp.

82. Nisus, Bp . (Emisus!) - ${ }^{\star} 238$. toussenellii, Verr. - . 259. sphenurus, Ruppell. - 240. polyzonoides, Smith. - 241. tachiro, Daud. - 242. badius, Gm. - 243. dussumieri, Temm - 244. virgatus, Temm.

85. Urospizia, Taup. - 245. approximans, Vig.246. torquatus, Cuv. (cruentus, Gould.) - 247. Iricolor, Vieill. (radiatus, Cuv.)

84 . Sparvius, Bp. (Eusparvius!)

a. Sparvius, Iip.-248.trinotatus, Temm. - 249. griserceps, Temm. - 250. hyogaster, Mull. (rufitorques, Peale.) Voy. P. sud.t. 22.

1. Tachyspizia, Kaup. - 251. soloensis, Horsf.

c. Scelospizin, Kaup. - 252. francisca, Smith.

8. Accipten, Br. - 253. nisus, L. - 2\%4. madagascariensis, Verr. - 25. g gularis, Schleg. - 256 . fuscus, Gm. - 957. erythronemus, Gr. - 258. casta nilius, Bp. - 259. chionogaster, Raup. - 260. ruliventris, Smith.

\$6. Jemspran, Kamp. - 261. Linus, Lath.-262? fonlanicri, BP. - 265. minullus, Dand.

37. Micnonsus, Gr. - 264. gabar, Dand. - 20in. gaInrims, Sundev. - 266. niger, Vieill. 
Sublam. 25. Cincine.

p. Circex.

88. Cincus, Lacép. - 267. æruginosus, L. (rufus, Gm.) 268? mulleri, Henylin. (Africa or. Constautinopol?) 259. gouldi, $B$ p. - 270. ranivorus, Gould. 271. spilonotus, Traup.

89. Spizacircus, Kaup. - 272. macropterus, Vieill. (leucophrys, Vieill. - gularis? Temm.) - 275. histrionicus, Quoy.

90. Strigiceps, $B \boldsymbol{p}$.

a. Spilocircus, Kaup. - 274. jardinii, Gould.

b. Pterocircus, Kaup. (Glaucopteryx, Kp. 1844, nec Hubn. 1816.) - 275. cineraceus, Montag. - 276. swainsoni, Smith. (herbecola? Tickell. - superciliaris, Smith.) c. Strigiceps, Kaup. - 277. cyaneus, L. - 278. hudsonius, L. - 279. melanoleucus, Gm. - 280. acoli, Daud. - 281. maurus, Temm.

\section{Subfam. 26. Polyborine.}

q. Ibycterea.

91. Etotnolichis, Kaup. (senex, J. E. Gr.)-- 282. australis, Gm. (nova-zelindiæ, Temm.)

92. Iвүстеr, Vieill. - 283. aquilinus, Gm. (nudicollis, Daud.)

95. Daptrius, Vieill. - 284. ater, Vieill. (aterrimus, Temm.)

94. Milvago, Spix. - 285. chimango, Vieill. (pezoporos, Meyen.) - 286. chimachima, Vieill. (degener, Ill.)

95. Phalcobenus, Orb. - 287. montanus, Orh. (megalopterus, Meyen.) 
r. Polyborece.

96. Polyborus, Vieill. - 288. brasiliensis, Gm. (vulgaris, Vieill. - plancus, Lath.)

\section{s. Polyboroidea.}

97. Polyboromes, Smith. - 289. typus, Smith.290. radiatus, Scopoli. (madagascarieusis, Daud. - gymnogenys, Temm.)

\section{Familia 9. GYPOGERANIDE.}

\section{Subfam. 27. Grpogeranina.}

98. Gypogeranus, IIl. (Serpentarius, Cuv.) - 291. serpentarius, Gm. (secretarius, Scopoli. - reptilivorus, Daud.)

Familia. 10. STRIGID AE.

Subfam. 28. Sthigina.

a. Strigea.

99. Strix, $\boldsymbol{L}$.

a. Meyastrix, Kaup. - 292. tenebricosa, Gould.

b. Dactylostrix, Kaup. - 293. personata, Gould. 294. castanops, Gould.

c. Strix, Kaup. - 295. delicatula, Gould. - 296. javanica, Gm. - 297. flammea, $L_{-}$- 298 . africana, Bp. - 299. poensis, Fraser. - 300. thomensis, IItrll. - 301. perlata, Licht. - 302. punctatissima, Gr. - 503. pratincola, Bp.

d. Scelostrix, Kaup. (Glanx. Blyth, nec Botanic.) 304. candida, Tickel. - 505. capensis, Smith.

100. Phonnus, Geoffr. - 306. badius, Horsf.

b. Ululea.

101. Uluhs, Cuv. - 307. cinerea, Gm. - 508. lappo. nica, Rel\%. - 509. nebulosa, Gm. 
102. Ptrnx, Blyth. - 310. uralensis, Pall. - 311. fuscescens, Temm

103. Nyctale, Brehm. - 512. funerea, L. - 313. ricliardsoni, Bp. - 314 . kirtlandi, Cassin. Ђ̈. acadica, Gm. - $316^{\text {P }}$ siju, Orb.

\section{c. Syrniece.}

104. Syrnium, Sav. - 517. aluco, L. - 518. woodfordi, Smith. - 319. nivicolum, Hodgs.

105. Macabra, Bp. - 320. hylophila, Temm. 321. fasciata, Vieill. - 322. suinda, Vieill. 325. melanonota, Vieill. - 324. cayanensis, Gm. 525 albigularis, Cassin.

106. Bulaca, Hodgs. - 326. indranee, Sykes.327. sinensis, Lath. - 328. ocellata, Less.

107. Mrrtha, Bp. - 329. pagodarum, Temm. 330. leptogrammica, Temm. - 531. sumatrana, $\boldsymbol{B} p$. 108. CicGaba, Wagl. - 532. huhula, Daud. (lineata. Shaw.)

109. Pulsatrix, Kaup. - 333. torquata, Daud. 534. major, Bl). (pulsatrix, Wied. - melanota, Aliq.)

110. Gisella, Bp. - 335. lathami, Bp. 1831. (harrisi, Cassin.)

\section{Subfam. 29. Ulubink.}

\section{d. Oteix.}

111. Orus, Cuv.

a. Otus, Kaup. - 3566. vulgaris, Flem. - 337. americanus. Gm. (wilsonianus, Less.) - 338. zonurus, Gr.

b. Rhinoptynx, Kaup. - 339. mexicanus, Gm.

112. Nrctalors, Wagl. - 340. stygius, Wagl. (l)rasiliensis, Less. - melanopis? Licht.) - 341 . siguapa, Orb. (granmicus? Gosse.) - 34\%. midas, Licht.

113. Brachyutus, Boie. - 545. agolius, Pall. - 
T4 palustris, BP. - 545. gallapagoensis, Gould.-

*546. sandwichensis, $B p$.

14. Phasmoptrax, Taup. - 547. capensis, Smith.

\section{e. Bubonea.}

115. Bubo, Cuv. - 548. maximus, Sibb. - 549? alhrniensis, Aldrov. - 550. sibiricus, Eversm. *551. confucius, $\mathbb{R}$ p. - 552. capensis, Dand. 555. cincrascens, Guérin. - 50ّ4. bengalensis, Franklin. - 355. virginianus, Gm. - 356. magellanicus, Gm.

116. Nisuelda, Bp. - 537. maculosa, Vieill. - 5058. dilIoni, O. des Murs. - 559. madagascariensis, Smith.

117. Megaptrnx, Bp. - 360. crassirostris, Vieill.

18. Nyctaetus, Is. Geoffr.-561. lacteus, Temm.562. verreauxi, Bp. - 563 ? sultanus, Less. (lacteus, Rupp.)

119. Urrus, Ilodgs. - 364. coromanda, Lath. 565. orientalis, Horsf. (pectoralis, Jerd.)

120. Ascalapha, Is. Geoffr. - 366. savignyi, Geoffr.

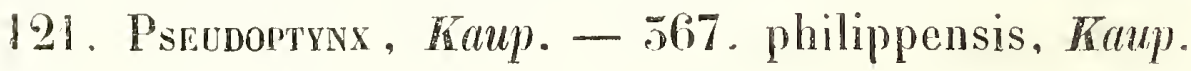

\section{Subfam. 50. Surininat.}

\section{r. Scopex.}

122. Kerupa, Less. - 368. ceylonensis, Gmel. 589. javensis, Less. - 570. llavipes, Hodgs.

125. Lopnostnix, Less. - 571. cristata, Dand. (griseala, lath.)

124. Pruropsis, Kaup. (Ephia les, Bp., nee Schrank, 1802.)

- 372 . Ieucolis, $S w$. - 5750 . megalolis, $G r$.

12\%. Acxurs, Homp. - 77\%. gymnopodus, Gr. 77.5? Indipes, Vieill. (Ois. Am. 1. 16.) 
126. Asio, Schlegel. (Megascops, Kaup.) - 376. 110-vius, Gm. - 377. atricapillus, Cuw.-378. brasi!iensis, Bp. - 579. watsoni, Cassin. - 580. 10. photes, Less. - ? trichopsis, Wagl.

127. Lenruus, Bp. - 381. semitorques, Schleg. 382. magicus, Mull. - 383. indicus, Gm. (lempiji, Gr.) - 584. lettia, Hodlgs. - 585. sagittatus, Cassin. ('eltoides? Jerd.) - 586. noctula, Reinw. (javanicus, Less.) - 387. mantis, Mull.

128. Scops, Sw. - 588. zorca, Gm. - 589. capensis, Smith. - 590. senegalensis, Sw. -- 591. leucopsis, Hartl. - 592. hendersoni, Cassin. - 593. pennatus, Hodgs. - 394. sunia, Hodgs. - 595. japonicus, Temm. - *396. kamichatchensis, Verr.

129. Pisonhina, Kanџ. - 597. rutilus, Pucheran. 398. manadensis, Quoy. - 599. novæ-zulandiæ, $\boldsymbol{B}$ ).

\section{g. Athenew.}

130. Scotopelia, Bp. - 400. typica, Bp. (Strix peli, Mus. Lugdunens.)

151. Nisox. Hodgs. - 401. squamipila, Bp. - 402. guteruhi, Mull. - 405. hirsuta, Temm. -- 404. borneensis, Schlegel. — 405. japonica, Schl. - *406. madagascariensis, Bp. ... * 407. philippensis, Bp. 408. forsteni, Bp. - 409. superciliaris, Vieill.

132. Pholeoptynx, Kan!. - 410. cunicularia, Molinu. - 411 . hypogaa, Bp. - 412 ? dominicensis. Vieill.

133. Arhene, Boie. - 415. noclua, Retz. (passerini, Temm. an Lath? nec $L$, -414 ? indigena, Brehm. 415. persica, Vieill. (meridionalis, Risso. - bactriana, Hutton. - numida, Levaill. jun._pharaonis, de Müll. - nilo. (ica, Wurtemb.) - 416 . brama, Temm.

134. Gymnası, Bp. - 417. nudipes, Dand. (Nochaa milipes, Lembeye, Aves Cub., 1. 4. fig. 2.) Mus. Paris. 
h. Ieroglancex.

155. Ieroglaux, Kaup. - 418. connivens, Lath. 419. strenua, Gould.

156. Rimabdoglaux, Bp) - 420. rufa, Gould. - 421. humeralis, Hombr. et Jacq. - 422. variegata, Quoy et Gaim. - 425. jacquinoti, Bp. ex Hombr. (All. ir. niala! Pucheran.)

137. Sceloglaux, $\boldsymbol{K}(1 \mathrm{u})$. - 424. alhifacies, $G r$.

158. Spiloglaux, Kaip. - 425. boobook, Lath. 426 ocellata. Hombr. et Jacq. - 427. maculata, Viy. - 428. marmorata, Gould. - 429. novæ-zelandix, Gm. - * 450. theomacha, B1) - 451. punctulata, Quoy et Gaim. - 452 fusca, Vieill.

159. Tenioglaux, $\boldsymbol{K}(u$ ) - - 455. castanoptera, Horst. (spadicea, Reinw.) - 454. malabarica, Blyth. (castmoptera, Jerd.) - 4555. castanotus, Blyth. (castanoptera, Bl., nec Auct.) - 456. radiata, Ticliel. - 457. cuculnides, Viy.

- 140. Suithiglaux, Bp. - 458. capensis, Smith.

141. Тænортухx, Kan\%. - 459. brodiei, Burton. 440. sylvatica, Muller.

142. Phalenopsis, Bp. - 441. nana, King. - 442. leucoliema, Bp. ex Hombr. et Jacq. (nana, Puch.) 445. infuscata, Temm. (passerinoids, Temm.) 444. ferruginea, Wied. - 445. minutissima, Wied. - 446. phalænoides, vieill.

140̄. Microglaux, Kall). - 447. perlata, Vieill. 448. licua, Licht.

\section{i. Surniece.}

144. Nrctes, Steph. - 449. nivea, Daud. ('andida, Lath.) 4/5\%. Surias, Dumér. - 450. ulula, L. (funerea, Lath.) 146. Gilauchond, Boie. (Microptyux, Kaup.)-451. passerina, L. (pusilla, Daud.-pygmaa, Bechst.-acadica, Temm.)

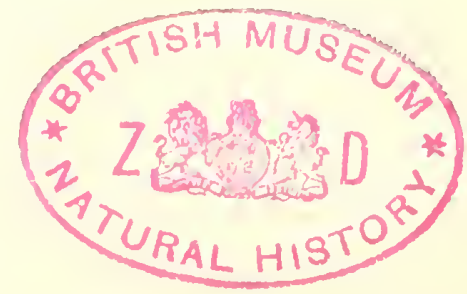


Extrait de la Revoe et magasin de zoozogie.

IN 7 . -1853 .

\section{MONOGRAPHIE DES LANIENS}

Par le Prince Ch. Wo BONAPARTE.

Fragment d'une leltre de $\boldsymbol{M}$. Ph. Defilippi à Son Altesse le prince Bonaparte.

N. Brun Rollet, négociant savoyard résidant à Kar-loum, ayant poussé par le Nil Blanc une excursion jusquie entre le quatrième et le troisième degré nord (latitude) dans l'Afrique orientale, a bien voulu faire profiter son pénible voyage au Musée de Turin, qu'il vient d'enrichir d'une belle suite d'oiseaux du pays qu'il a visilé. Si j'ai été trompé dans mon espoir d'y trouver quelques individus du Balceniceps rex, j'ai eu du moins la satisfaction de découvrir dans ce précieux envoi quelques espèces nouvelles et d'autres bien connues, mais non mentionnées dans le dernier résumé de l'ornithologic de l'Afrique orientale de notre ami Rüppel (System. uebers. Vôgel nord-ost Afrika's). Voici le catalogue de ces espèces :

Micronisus monogrammicus, Temm.

Polihierax semitorquatus, Smith.

Athene perlata, Vieillot.

Irrisor Cabanisi, Defilippi. - Magnitudine, forma, pictura, Irrisori minori, Rüpp. valde affinis; sed absque remigum maculis albis.

Muscipeta cristata, Gmel. 
Lamins dealbatus, Defilippi. - Supra pallide cinereus : uropygio scapularibusque sensim albis : rostro, vitla a naribus orta parum ultra regionem paroticam protensa, tectricibus alarum et remigum tertii ordinis maxima parte, nigris : remigibus primariis ad hasim per dimidiam rtem, secundariis pogonio interno et limbo lato apicali, albis; ceterum fuscis : subtus pure albus.

Cauda gradata : rcctricibus angustatis; duabus externis in utroque latere albis scapo fuscescente; tertia macula magna oblonga fusca in pogonio interno; quarta nigra, apice albo; quatuor medianis totidem nigris: pedibus luscis. - Longitudo 0 m. 20.

Specimen in R: Musco Taurinensi, jam extabat a Tripoli.

Lanius macrocercus, Defilippi. -- Supra cinereus, infra albus: vitta magoa frontali per oculos ad latera colli totius ducla: alis nigris, excepta remigum primi et secundi ordinis dimidia parte basim versus alba: plumis axillaribus nigris in plerisque (pro atate aut sexu?) chocoladinis : cauda longa gradata ; rectricibus dilatalis dimidia parte basali alba, apicali nigra; duabus intermediis nigris ad basim tantum cinereis, transversim nigro-lasciatis; pedibus nigris. - Longitudine, $0 \mathrm{~m} .24(1)$.

Corvinella cissoïdes, Vieillot.

Oriobhs larvatus, Lichienst.

Corospiza simplex, Lichtenst.

Amadina cantans, Gmelin.

Buphaga Africana, Gmelin.

Indicalor albirostris, Temm.

Pogonias Rolleli, Defilippi. - Magniludine P. Dubii: differt collo toto pectoreque cum capite et dorso uni-

(1) Voyez, ì la fin de l'article, la note ajouce par Son Aliesse le prince lionaparte. 
coloribus nigro-violaceis sericeo resplendentibus; plumis ventris lateralibus stramineis rarissime nigro flammulatis ; rostro valde convexo; sulco maxilla profundissimo, mandibula lævi; pedibus fuscis. Ceterum cum $\boldsymbol{P}$. Dubio confert (1).

Laimodon leucocephalus, Defilippi - Capite, collo toto, pectore, dorso infimo, uropygio crissoque albis : dorso supremo, tectricibus alarum ventreque fuscis albicanti longitudinaliter flammulatis : remigibus rectricibusque fușcis, illis margine interno albescenti. Rostro nigro levissime unisulcato; pilis mystacalibus exiguis albis. Pedibus nigrescentihus. - Longitudine, 0 m. 16.

Parra Africana, Latle. Juv. - Pectore ventreque albis.

Ardea ardesiaca, Wagl.

- atricollis, Wagl.

Ciconia umbellata, Wagl.

Je saisirai cette occasion pour regretler, avec tous les ornithologistes, l'interruption de voire excellent Conspectus avium; j'espère, mon prince, que vous ne laisserez pas incomplet cet ouvrage, qui devrait trouver des imitateurs dans les autres branches de la zoologie.

Turin, ce 19 juin 1853.

Ph. Defilippi.

\section{Note.}

Cette espèce ne diffère pas de celle figurée planche 8 du voyáge de Lefèvre en Abyssinie, et que M. O. Des Murs a nommée, p. 89, Lanius excubitorius; elle a été aussi nommée Lanius princeps par M. Cabanis, et se trouve, ainsi que $\boldsymbol{L}$. dealbatus, Defilippi, exposée de-

(1) Celte belle espèce est la seconde du genre Pogonias, comme il convient de le restreindre sous le nom imposé par Iiliger, et qui, sous cctte forme, n'avait jamais été employé.

Charles L. Bonafarte. 
puis longtemps au public sans aucune dénomination dans les galeries du Muséum d'histoire naturelle de Paris.

J'espère que mon savant ami Defilippi ne me saura pas mauvais gré de cette note, et que surtout il ne voudra pas la confondre avec celle insérée à la page 171 de ce volume. A cette occasion, j’ai lait subir une revue sévère à toute la famille des Laniides, qui doit être considérablement restreinte pour former, avec les $\boldsymbol{E} d o$. liides, les Oriolides et les Artamides, le groupe supérieur des Dentirostres. Les Laniides ne doivent être constitués que par les sous-familles des Laniens et des Malaconotiens. Les vrais Laniens se composent des genres Lanius, Enneoctonus, Eurocephahus, Corvinella, et Basanistes (ou pour mieux dire Urolectes, Cabanis), Sigmodus lui-même devant prendre rang parmi les $M a$ laconotiens.

Voici, d'après mes notes et l'étude approfondie des richesses exposées et enfonies du Muséum du Jardin des Plantes, l'énumération des espèces de Laniens en commençant par celles de véritable Lanius, que je crois devoir subdiviser ultérieurement en plusieurs petits groupes ou sous-genres. Charles L. Bonaparte. 
Moxographie des Laniens, par Charles L. prince Bonaparte.

SUBFAM. 85. LANIINE.

Genus I. LANIUS, L. (Collyrio p. Merr. - Collurio, Vig.)

Subgenus I. Lanius, Bp. (1).

A. Europcei.

1. Lanius excubitor, L. (major, Brehm. - cinereus, Leach, ex Br.), pl. enl. 445. - Roux, Orn. Prov. t. 152. - Gould, Eur. t. 66. ex Eur. s. et media.

Dilute griseus, capistro concolore, dorso albo-marginato; subtus cum speculo alarum duplici, magıo, albus : remigum secunda sextam æquante; tertia, quarta et quinta omnium longissimis : rectricibus latis, exterioribus fere omnino albis.

2. Lanius meridionalis, Temm. pl. col. 143. - Roux, Orn. Prov. t. 152. - Gould, Eur. t. 66. ex Eur.m. occ.

Fusco-cinereus, capistro albido; subtus roseus : superciliis, gula, crissoque albis : speculo alari unico, circumscripto : rectricibus exterioribus partim albis.

(1) Lanius minor, Gm. (italicus, Lath., - vigil, Pall. - longipennis, Blyth.) est un Enneoctonus!

L. subicus, Licht. (brubru, Sibthr.) constitue à lui seul mon nouveau genre LeucoMetopon.

L. AFer, Lath. (signatus, Shaw) et L. pexdens, Lath., Lev. Afr. t. 66.1, sont des espèces très-douteuses.

L. melanurus, Licht. est synonyme de son B. cissoldes (melanoleucos, Smith), bien différent de celui de Vieillot, qui doit rester seul dans le genre Corvinella, tandis que l'oiseau de Lichtenstein est le type du genre Urolestes; Cabanis, noin substitué à BasanIS TES, préoccupé. 


\section{B. Africuni.}

3. Lanius algeriensis, Less. Rev。zool. 1839, p. 134. ex Afr.s.

Cincreo-plumbeus, capite obscuriore, capistro nigro, superciliis concoloribus : subus cinereus : speculo alari unico circumscripto : cauda elongata, rectricibus angustioribus, externis dimidiato albis : rostro robusto.

4. Lanius excubirorius, $O$. Des Murs (princeps, $C a$ banis, - macrocercus, Defilippi) Lefewr. Voy. Abyss., p. 89. 1.8. ex Afr. or. et centr.

Griseus; subtus candidus : fronte lata et vitta transoculari protracta, alis candaque nigerrimis : speculo alari vix ullo : rectricibus omnibus ad medium usque albis.

5. Lanius dealbatus, Defilippi (Mus. Paris ex Nilo albo, Arnaud, 1845!!!) Fig. mulla. ex Afr. or. et centr.

Griseus, fronte concolore; subtus abbo-poseus : speculo alarum latissimo albo : cauda brevi, rectricibus angustis, acutis, mediis nigris, cxtcrnis albis, rachide tantum nigro.

\section{Asiatici.}

6. Collofio mathora, Sylkes (L. burra, J. Gr., - excubitor, var. C. Lath., - excubitor, Franklin, - magnirostris, Less., - Lanius lathora, Blyth.), Hardw. Ill. Ind. zool. 1. 32.1, 2 el 1. 33. 3. ex India univ. excl. liengal. infer.

Griseus, capistro uigro; subtus albus : remigibus, rectricibusque lateralibus magne ex parte allsis.

7. Lanius auchen, Pucheran, Mus. Paris. ex Persia Aucher, 1840.

Similis precedenti, medius quasi inter lathoram ex excubitorem; serl obsrurior et dorso sine albo; cauda longiore, rectricibus 


\section{-7 -}

angustioribus et cum renigibus minus albo variis: remigibus secundariis brevissimis !

\section{Americani.}

8. Lanius septentrionalis, Gm. (excubitor, Wilson,-borealis, Vieill.) Ois. Am. s. t. 50. - Wils. Am. Orn. t. 5. 1. - Sw. Faun. Bor. Am. t. 33, - Aud. Am. t. 192. ex Amer. bor.

Spurco-cinereus, capistro albido, macula postoculari nigra cir'cumscripta : subtus albus undulis plus minus cinereis vix evanescentibus; speculo alarum albo restricto; remigun secunda breviore quam sextam; quarta omnium longissima : rectricibus latis, lateralibus apice tantum albis : mandibula basi pallida.

9. Lanius ludovictanus, L. (ardosiaceus, Vieillot, carolinensis, Wils., - excubitorides, Sw.), Ois. Am. s., t. 5l, -Wils. Am. Orn, t. 22. 5-Faun. Bor. Am. t. 34, - Aud., Am. t, 57. ex Am. s.

Fusco-cinereus, capistro concolore vix nigricante, superciliis albis : subtus albo-griseus : speculo alari albo circumscripto; remigum secunda sextam æquante; tertia omnium longissima: rectricibus angustis, lateralibus apice late albis : rostro nigerrimo.

10. Lanius elegans, Sw. Faun. Bor. Am. Fig. nulla, ex Am. s.

Pure cinereus, capistro concolore, linea superciliari vix alba : subtus alb:dus; pectore sub-gri eo-rosaceo parum undulato; alis albo-variis ob speculum conspicuum et latos remigum margines apicales; tertia et quarta omnium longissimis : cauda nigerrima, rectricibus lateralibus apice lato albis : rostro nigerrimo. 



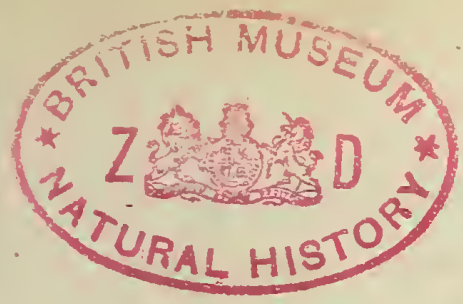

\section{DESCRIPTION}

B'UNe

\section{ESPËCE NOUVELLE D'OISEAU-MOUCHE,}

Par Mil. J. BOLRCIER et E. MULSANT.
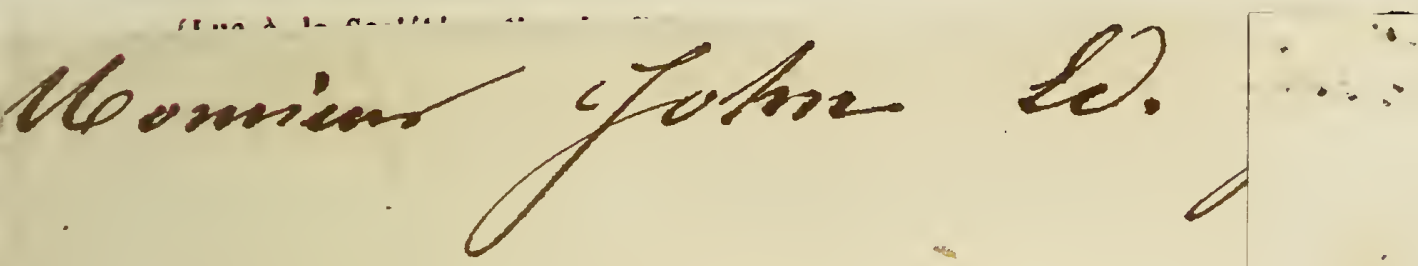

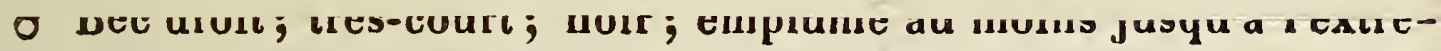
mité des narines : celles-ci, couvertes. Téte allongée. Dessus du corps revêtu de plumes d'un gris enfumé, légèrement bronzées sur la tête et sur les couvertures des ailes : dos et croupion d'un bleu violet. Ailes moins longuement prolongées que la queue; d'un brun violacé. Queue fourchue; à dix rectrices largement barbées; d'un bleu vert ou d'un vert bleuâtre. Gorge parée d'une sorte de rabat ou de plastron très-allongé et subgraduellement rétréci, formé de plumes écailleuses, áont les inférieures sont longues et étroites, d'un vert émeraude très-brillant sur sa parlie antérieure, passant à l'améthiste bleu vers sa partic inféricure, bordé dans sa périphérie de plumes brunes et soyeuses. Ventre d'un gris enfumé. Page inférieure de la queue d'un bleu d'acier. Couverture sous-caudale formée de plumes d'un blanc sale ou cendré, ornées dans leur milieu d'une tache d'un brun ou bleu d'acier. Tarses noirs, dénudés.

ㅇ Diffère du $\sigma^{\prime}$ par l'absence du plastron métadlique sous-jugulaire.

Longueur totale $0^{\mathrm{m}}, 125$; bec $, 0^{\mathrm{m}}, 013$; ailes, $0^{\mathrm{m}}, 070$; queue : rectrices exlernes, $0^{\mathrm{m}}, 050$ : rectrices interues, $0^{\mathrm{m}}, 030$.

Nous avons dédié cctte espèce à l'honorable Édouard-Henri Stanley, fils du lord du même nom, mombre du parlement anglais. Puisse cet hommage lui rappeler quelquefois son passage à Quito, en 1849 ! Puisse-t-il lui inspirer les goûts de 

B'UNe

\section{ESP ËCE: NOUVELLE' DNOISEAU-MOUCHE:}

Par UII. J. BOLRCIER et E. MULSANT.

(Lue à la Soclété nationale d'agriculture, d'histoire naturelle et des arts utlles de Lyon, dans la séance du 24 mai 185\%.)

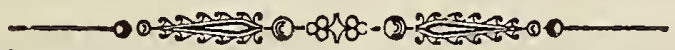

\section{TROCEILUS STANLEYT.}

$\sigma^{\circ}$ Bec droit ; très-court ; noir ; emplumé au moins jusqu'à l'extrémité des narines : celles-ci, couvertes. Tète allongée. Dessus du corps revêtu de plumes d'un gris enfuıné, légèrement bronzées sur la tête et sur les couvertures des ailes : dos et croupion d'un bleu violet. Ailes moins longuement prolongées que la queue; d'un brun violacé. Queue fourchue; à dix rectrices largement barbées; d'un bleu vert ou d'un vert bleuâtre. Gorge parée d'une sorte de rabat ou de plastron très-allongé et subgraduellement rétréci, formé de plumes écailleuses, áont les inférieures sont longues et étroites, d'un vert émeraude très-brillant sur sa partie antérieure, passant à l'améthiste bleu vers sa partie inférieure, bordé dans sa périphérie de plumes brunes et soyeuses. Ventre d'un gris enfumé. Page inférieure de la queue d'un bleu d'acier. Couverture sous-caudale formée de plumes d'un blanc sale ou cendré, ornées dans leur milieu d'une tache d'un brun ou bleu d'acier. Tarses noirs, dénudés.

q Diffère du $\sigma^{\prime}$ par l'absence du plastron métallîque sous-jugulaire.

Longueur tolale $0^{\mathrm{m}}, 125$; bec, $0^{\mathrm{m}}, 013$; ailes, $0^{\mathrm{m}}, 070$; queue : rectrices externes, $0^{\mathrm{m}}, 050$ : rectrices internes, $0^{\mathrm{m}}, 030$.

Nous avons dédié cette espèce à l'honorable Édouard-Henri Stanley, fils du lord du même nom, membre du parlement anglais. Puissc cet hommage lui rappeler quelquefois son passage à Quito, en 1849 ! Puisse-t-il lui inspirer les goûts de 
son noble aieul, lord Derby, et ofrir à ce dernier un témoignage de notre gratitude pour l'accueil gracieux que nous avons recu chez lui, l'un el l'autre, en 1847 !

Quel naturaliste n'a visité ou du moins n'a oui parler du magnifique château de Knowsley (1), et des merveilles du jardin zoologique qui l'entoure? Là, vivent au nombre de plus de mille, et sans doute fort étonnés de se trouver si rapprochés, les mammifères et les oiseaux les plus rares de toutes les parties du monde: les premiers, enfermés dans des bâtiments spacieux, ou parqués dans des enclos séparés par des treillis de fer et par des bosquets : les seconds, soit logés dans des pavillons où ils trouvent la température élevée qui leur est nécessaire, soit dispersés dans des volières, la plupart assez vastes pour permettre aux arbres de moyenne grandeur de s'y développer.

L'oiseau-mouche de Stanley (2) paraît ne s'éloigner jamais des limites les plus élevées de la végétation. Le fragment suivant de la lettre de $\mathbf{M}$. Bourcier servira à faire connaître les lieux dans lesquels il a été récemment découvert par ce naturalisie.

“ J'ai entrepris, il y a quelques'jours, avec deux Indiens, une ascension au Pichincha, volcan qui n'est situé qu'à trois lienes et demie de Quito, à l'onest nord-ouest de cette ville, mais auquel on n'arrive ordinairement qu'après sept à huit heures de marche. Une partie de la route peut se faire trèsfacilement à cheval, mais lorsqu'on est parvenu à une certaine hauteur, it faut abandonner ses montures jusqu'au retour. Liisolement où l'on se trouve permet d'altacher ces animaux dans les champs, sans avoir à craindre les voleurs. De là, pour arriver au point culminant, il nous fallut escalader des amas

(1) Pres du village de Iluyion, à deux lienes de Liverpool.

(2) Il doit itre placé près des $T$. heteropogon, Borsonv., el T'. Mernani, De I.Aitr. at J. listric. 
de pierre ponce disposés en pente très-raide. Mais comment vous dire les sentiments divers, les impressions vives et profondes que j'éprouvai après avoir triomphé de ces obstacles? Comment vous peindre la magnificence du tableau que nous avions alors sous les yeux! Figurez-vous deux cavités cratériformes séparées par un mur trachytique, au sein desquelles sont ouvertes près de quarante bouches vomissant de la fumée! Nous étions au-dessus du niveau des mers, à une hauteur pareille à celle du Mont-Blanc; nulle trace de végétation autour de nous : c'était le désert avec sa majesté et son silence. Le condor seul, ce roi des solitudes élevées, planait au-dessus de ces lieux déserts son éternel domainc. A nos pieds se montraient les vastes effondrements dont la distance nous dissimulait la profondeur. Ils se sont formés sans doute à la suite des éruplions épouvantables dont l'histoire et la tradition ont conservé les douloureux souvenirs. Malheureusement nous nous étions dirigés vers le point le plus élevé; il fallut nous détourner de près de trois quarts de lieue pour trouver une pente moins longue; elle était néanmoins encore de 400 à 500 mètres. Il me tardait d'arriver au fond de ce cirque désert, d'approcher de plus ou mòins près de ces soupiraux qui préservent peutêtre le pays des colères nouvelles du volcan, et surtout d'explorer sous le rapport ornithologique ces champs trop rarement visités! Mais que de peines et de dangers avant d'être rendus! Pour descendre ces flancs abruptes, on est obligé soit de s'appuyer sur ses mains, soit parfois de se laisser glisser. Souvent, les pierres calcinées sur lesquelles on pose le pied se pulvérisent sous le poids du corps qui les foule, font perdre l'équilibre à leurs voisines, en attirent d'autres à leur suite, et occasionnent des éboulements plus ou moins considérables qui menacent la vie du voyageur.

“ Après quatre heures de fatigues adoucies par l'espoir de prochaines jouissances, nous touchons au but tant désiré. Le 
cratère dans lequel nous nous trouvions a une forme circulaire ou un peu ovale; il est entouré d'une sorte de mur de trachyte d'une élévation uniforme, coupé du côté de l'occident, pour le passage des eaux qui se jettent dans l'Esméralda, et de là portent leur tribut à l'océan Pacifique. Dans cette sorte d'enclos surgit un cône élevé d'où sortent de nombreuses bouffées d'acide sulfureux!, et d’où s'échappe avec un certain bruit de la vapeur d'eau. Dans quelques-uns de ces soupiraux le soufre se condense en cristaux. Dans divers endroits, le sol est couvert de ponces pulvérisées ou d'une cendre noire, qui rend timide le pied désir'eux de s'approcher de ces cheminées. La terre présente des crevasses dont l'œil ose à peine mesurer la profondeur; quelques-unes doivent avoir au moins 200 mètres; elles recueillent les eaux des pluies et de la fonte des neiges, et forment le lit des ruisseaux qui s'échappent ver's l'occident. Ces ravins ou quebrados, comme les nomment les Indiens, obligent à des détours considérables et font perdie un temps énorme; les moins profonds présentent leurs flancs tapissés de verdure et ornés de divers ar'üstes. C'est là que jai eu le plaisin de me procurer l'Oiseau-mouche de Stanley, char. mante créature qui butinait sur le chuquiraga insignis, décrit par l'illustre Alexandre de Ilumboldt (1), dont le Pichincha et tous les lieux des environs semblent avoir retenu le nom. L'oiseau-mouche de Stanley parait être le courtisan assidu de celte plante, conjointement avec l'Oiseau-mouche Pichincha, qui lui fait une guerre continuelle.

« Il m'aurait fallu huit jours pour explorer complètement ces lieux sanvarges; mais comment y transporter la nourriture nécessaire? Comment surtout pouvoir espérer, pendant une semaine, une température favorable? J'ai été, sous ce dernicr rapjort, favorisé au-delà de mes espérances. A une journée 
magnifique succéda une nuit superbe, pendant laquelle la lune dans son plein prêtait à ces lieux un charme féerique. Enveloppés dans nos ponchos, espèces de manteaux du pays, nous attendimes le jour, couchés sur des pierres et abrités par un rocher; malgré le peu de commodité du lieu, nous jouimes des douceurs d'un sommeil profond, sur ce sol menaçant, qui, dans un moment de caprice, pouvait nous lancer dans l'espace. «Le lendemain, des peines inouies nous attendaient pour regagner les rochers qui servent de barrière à cet enclos ; il nous fallut piétiner dans un terrain arénacé dans lequel nous enfoncions jusqu'aux genoux, avancer péniblement sur ce sable mobile qui fuit sous le pied comme celui des dunes, prendre pour appui des rocher's qui se brisent sous les doigts. Il faut la passion d'un naturaliste pour se vouer à de pareilles fatigues. Arrivés enfin après des heures longues et pénibles sur les degrés les plus élevés du versant opposé, nous nous trouvàmes bientôt dans les paramos, montagnes où l'on envoie paître les bœufs. Les vents s'y font sentir parfois avec tant de violence et $\mathrm{y}$ sont d'un froid si pénétrant, que déjà par deux fois j'étais arrivé jusqu'à ces champs sans me sentir le courage de poursuivre ma route. Nous reprîmes nos chevaux; grâce à ces animaux nous eûmes bientôt franchi les pentes boisées dans lesquelles croissent des Daturas d'espèces variées, puis les plaines plus riches qui conduisent à la ville. En rentrant à Quito toutes mes peines étaient oubliées; il ne me restait que le plaisir d'avoir fait pour la science une conquête nouvelle, et le souvenir des jouissances délicieuses que j’avais pu goûter.»

(Extrait des Annales de la Société nationale d'agriculture, d'histoire naturelle et des arts utiles de Lyon. - 1851.) 



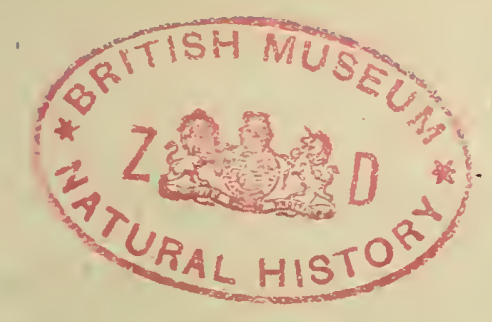

QUATORZIÈME ANARE. - OCTOBRE 1851.

\section{TRAVAUX INÉDITS.}

Essal d'une monographie du genre Picucule (Buffon), Dendrocolaptes (Hermann, Illiger ), devenu aujou rd'hui la sous-famille Dendrocolaptine (Gray, Genera of Birds), de la famille Certhiade de Swains.; par F. de Lafresiaye. - Suite. Voy. 1850, p. $95,143,27 \%, 569,417,588 .-$ 1851. $-145,517$.

$7^{\circ}$. Dendrocops Tordinus, Licht., Monogr. du genre Dendrocolaptes, $\mathrm{n}^{\circ} \mathbf{7}$, et Ciat. des doubles du Mus. de Berlin, $\mathrm{n}^{\circ} 150$.

"D. supra olivaceo-brunneus, fere unicolor, uropygio parum rufescente, iectricibus caulæ superis ferrugineis; pilei plumis in medio pallidiore vix conspicuè striatis; subtùs paulo pallidior, gutture grisescente, alis caudâque cinnamomeis; rostro recto, cultrato, livido, gonyde albâ. - Lorigit tota (ave arte farcto), 20 cent. 172 ; alæ plicatæ, 10 cent.; caudæ, 9 cent.; rostri a fronte, 2 cent. 1/3. - Habitat in Brasilià, Bahia. w

Cette espèce, voisine de la précédente par son système de plunage presque unicolore, a, commie elle, sur la tête des stries d'une teinte plus pâle et peu apparentes. Le brun olivâtre du dos prend une teinte un peu roussâtre sur le croupion, et ferrugineuse sur les suscaudales. Les rémiges secondaires sont d'un ferrugineux olivâtre, et la queue est, suivant l'usage, d'un brun canelle intense. Tout lo dessous est de la couleur du dos, mais plus pâle et sans maculatures. La gorge est d'un gris roussâtre pâle. Le bec, de couleur livide en dessus, a sa mandibule inférieure blanche, et ce dernier caractère la fait aisément distinguer de notre lyranninus, d'ailleurs notablement plus grand, $2^{\mathrm{e}}$ ś́RIE. T. III. Année 1851. 
et de plusicurs autres espèces voisines. La mandibule supérieure est légèrement infléchie à son extrémité, et l'inféricure suit un peu cette inflexion.

$8^{\circ}$. D. Fumigatus, Licht., Monogr.. no 8. - Grimpart enfumé, Levaill., Pronérops et Guêpiers, pl. 28. - Dend. fuligivosus, Vieill., Dict., vol. 26, p. 117.

a D. rostro recto, apice deflexo, valido, nigro, vittâ utrinque duplici supra et infra oculos pallidâ; capite corporeque immaculatis. - Longit. 8 pouces. - Habitat in Cayenna."

Telle est la diagnose de Lichtenstein.

Cette espèce, d'après Levaillant, serait remarquable, entr'autres caraclères, par deux bandes de couleur roux clair de chađ̧ue côté de la tête, l'une au-dessus, l'autre audessous de l'œill. Elle a le bec noir, blanchissant vers la pointe, terminé par un petit crochet; la gorge roussâtre; tout le dessous d'un brun roussâtre, aiminuant de vigueur vers le ventre; les pieds sont plombés. Elle habite Cay enne, selon Levaillant.

$9^{\circ}$. D. ATrirostris, Nob., Synops avium Amer., $2^{\circ}$ part., p. 12, et d'Orbigny, Voy., pl.54, f. 1 .

(D. supra totus brunneo-olivaceus, fere unicolor, tectricibus caudæ superis rufo-ferrugineis, alis caudâque cinnamomeis; pileo unicolore aut striis angustis paulo pallidioribus vix conspicue notato; vittì superciliari aut potius post-oculari pallicle rufâ; subtùs dorso concolor', gutture, colloque antico parum grisescentibus, pallido ita ut caput vix conspicue striolatis; rostro atro, recto, apice sensim curvato, uncinato et pallescente; subcaudalibus apice ferrugineis; tarsis dighitisque debiliopibus. - Longit tota (ave arte farcto), 20 cent.; alæ plicatæ, 10 cent. 1 læ; caudæ, 9 cent. 12. - Habitat in Boliviâ, Guarayos d'Orbigny et in Colombiâ. ఎ

Nous avons été longtemps incertain si nous ne devions point rapporter cette espèce bolivienne à la précédento (le Grimpart enfumó de Levaillant, de Cayenne); une seule particularité de coloration, jointe à la diversité d'habitat (C'ayenne et le Pérou), nous a fait douter de leur identité. 
C'est que Levaillant, et, après lui, Lichtenstein, décrivent le Grimpart enfumé comme ayant de chaque côté de la tête deux bandes claires, l'une au-dessus et l'autre audessous de l'œil, tandis que notre atrirostris de Bolivie et de Colombie n'en a positivement qu'une, et plutôt postoculaire que sur-oculaire.

10. D. Merdla, Licht., Monogr. - Mém. de l'Acad. de Berlin, 1818, n० 17, p. 208.

《 D. long. 7 pouces $1 \mathbf{1 2}$ rostro recto, compresso, brevi, apice deflexo nigrescente, gonyde albâ, corpore toto obscure guajacino, gulâ albâ. - Habitat in Cayenna."

Telle est la diagnose du docteur Lichtenstein; en voici une plus étendue que nous avons été à même de faire sur un individu rapporté au Musée par l'expédition Castelnaud.

"D. totus unicolor ; supra fusco-brunneus, alis, uropygio caudâque saturate cinnamomeis; subtùs olivaceo-brunneus, gutture albicante, abdomine crissoque viride cinnamomeis; rostro recto, parum apice curvato, maxillâ nigrâ, mandibulâ albâ aut flavescente, pedibus nigris, debilibus. - Longit. tota, 20 cent. 1/2; alæ plicatæ, 10 cent. 1 l3. - Habitat loco dicto les Missions de Sarayacu, ad summum Amazon. ")

Cette espèce, voisine, par sa coloration, des quatre précédentes, en diffère un peu par son bec plus étroit, quadrangulaire; par l'absence totale de taches roux clair sur la tête et le devant du corps, et par la tache blanc sale qui couvre chez elle toute la partie gutturale.

$11^{\circ}$. D. Meruloides, Nob.

Cette espèce, extrêmement voisine du Dend. merula, n'en diffère que parce qu'elle n'a pas, comme lui, la gorge blanche. Son croupion n'est que roussâtre, et non roux canelle, comme chez le merula; il en est de même pour la nuance de l'abdomen et de l'anus, et aussi de la queue, qui est d'un canelle plus foncé chez le merula. Le bec est, du reste, semblable de forme, tout droit, et pres- 
que quadrangulaire. Llle a été rapportée de la côte ferme par M. Beauperthuys.

Tel était le nombre des espèces à nous connues, lorsque nous adressâmes, il y a plus de six mois, à M. Guérin lo texte de cette dernière partie. Pendant ce long retard dans son impression, M. T.-C. Eyton, Esq. a publié, dans les Contributions to Ornilhology, 18.j, part. 4, qui vient de paraître, la description de trois espèces nouvelles de Picucules et d'un nouveau genre des Dendrocolaptince (le genre Deudrexetastes, Eyton).

Nous donnons, sous forme de supplément à notre travail, les descriptions de ces trois espèces et du genre, copiées sur celles mếne de l'auteur, nous réservant de les intercaler au milieu des nôtres, dans leur ordre respectir, dans une table générale des espèces que nous donnerons dans le prochain numéro, ainsi que leur traduction en français.

«Dendrocolaptes multistrigatus, Eyton. - D. rostro parùm arcuato valdè depresso cultrato; gulâ, maculis triangularibus capitis vittaque postoculari, flavo, rufis; singulis pennis pectoris latè, colloque superiore angustè medio eodem color e strigatis; abdomine pectoreque imo flavo-rufis singulis pennis quatuor striis: angustis atris et transversis notatis; illis pectore atro marginatis ; dorso brunneo-olivaceo, inferiore parte uropygio remigibus rectricibusque lætè cinnamomeo rufis his apicibus brunneo viridi vix tinctis; rostro mandibulà superione atıo inferiore ad basin corneo. - Long. corp. 10. 5; rosl. 1. 6; tars. 1. (pouces anglais).

This species of Dendrocolaples approaches nearer to I. platyrostris, Spix, in the form of the bill than to any other species, but has it more slender, not so broad at the base, but at the same time more depressed. The specimen from which the above description is taken is in Lord Derby's Museum at Knowsley.

“Picolatétes validirostris, Eyton. - P. rostro valido, ar- 
cuato, cultrato, ad basin palidè brunneo; capite brunneo-atro, pennis singulis in medio rufo-flavo latè strigulatis et nigro-fusco limbriatis, striis colli mediis staturâ inter illos capitis et dorsi ; gula genisque sordidè albis; pectore abdomineque pennis in medio parte eodem coloris fusco leviter fimbriatis; uropygio remigibus rectricibusque lætè cinnamomeo-rufis, remigibus externis apicibus brunneis viridi parcè tinctis; pedibus brunneo-corneis. - Long. corp. 8. 5 ; rost fron. 1.5 ; tars., 9 ; alæ, 4. 3.

The above may be easily distinguished from the other species of Picolaptes, by the greater length and strength of the bill. The specimen is in the Knowsley Museum.

"Picolaptes atripes, Eyton. - P. præcedente similis sed pennis medio parte lætè albis nigro fimbriatis, rostro breviore et gracilliose, et pedibus atris. - Long. corp. 8. 5 ; rost. fron., 1. 5 ; tars., 9; alæ, 4. 7. 1 l2.

This species approaches very nearly to the preceding one, but may be at once distinguished from it by the superior length of the wing, the slenderness of the bill, the black or very dark brown feet, and by the centre of the feathers being pure white instead of dirty white. A specimen is in Lord Derby's Museum and another in my own.

Il est facile de reconnaître que la première de ces trois espèces fait partie de notre deuxième section, les Dendrocolaptinées dépressirostres, et suivra immédiatement, sous le numéro $5^{\circ}$ (bis), la description de notre $5^{\circ}$ Dendrocops plalyrostris, $185 \%$, p. 526 , dont elle paraît différer par une taille et un bec surtout plus allongés, par une teinte inférieure d'un roux jaunâtre et non blanchâtre, et par des stries ventrales noirâtres plus nombreuses et plus étroites, etc., etc.

La seconde, Picolaptes validirostris, offre des rapports dans la force de son bec et sa coloration avec notre Picolaptes Souleyeti; et la troisième, le Picolajtes atripes, en offre, par sa maculature blanche, avec le Picolaptes leuco. gaster de Swainson; mais ces deux dernières ont le bec plus long que nos deux espèces décrites. 
"DENDREXETASTES, n. g. Generic Character. - Rostrum valioum, arcuatum, parùm cultratum, haud altior quam latum, naribus magnis vix ovalibus.

"Pedes tarsique $e_{\mathrm{i}}$ validi, cauda duabus rectricibus mediis longissimis.

“ Dendrexetastes capitordes, Eyton. - D. capite, dorso abdomineque brunneis, pectore, gulâ colloque posteriore, pennis medio parte latè albis, atris, dein brunneo fimbriatis, gulâque brunneu solummodo fimbriatâ; uropygio, reciricibus remigibusque latè rufo-cinnanomeis illis apicibus brunneo-viridi exteriore latere propillatis. -... Long. corp. 9; rost. fron., 1. 2; tars., $11 ;$ ala, 4.

"From the form of the bill in this curious genus it might at first sight be supposed "to be related to the Capilonnice; but being destitute of bristles at the base of the bill, and also the toes being placed, three in front and one behind, point out its true position to be among the Dendrocolaptince. It also agrees with the latter family in the structure of the tail and in the style of the plumage. The specimen from which the above description is taken is in the Knows. ley Museum, and was purchased from Mr. Leadbeater, the locality in unsknown.

SuR deux nouvelles espèces de Tyrans d'Amérique, par M. F. DE LAERESNAYE.

Au milieu des nombreuses espèces de Tyrans qui peuplent les deux Amériques, il en est deux remarquables par leur taille et leur conformité de coloration, mais en même temps par la grande différence dans la forme de leur bec; Ce sont: 10 le Bienteveo ou Puitanga d'Azara, ou le Lanius sulphuralus de Gmelin, enl. 249, sous le nom de Geay à ventre jaune, de Cayenne, ol dont la Bécarde à ventre jaune, enl. 296 , qui n'a pas de jaune sur le milieu de la tête, nous paraît être le jeune âge, espèce à bec droit, robuste, comprimé, crochu à l'extrémité. $2^{\circ}$ le Nei-nei d'Azara, enl. 
212, appelé par Vieillot le Bentaveo lanius pitangua, à tort. puisque c'est au premier qu'appartient ce nom de pays, selon Azara, qui les a très-bien distingués dans ses deux descriptions. Celui-ci se distingue, au premier abord, du premier par un bec singulièrement élargi, déprimé, arqué en dessus, et ayant un peu la forme d'un bateau renversé. Dans ces derniers temps, ces deux espèces sont devenues les iypes de deux genres nouveaux. On a formé, avec la première, le genre Saurophagus, et, avec la seconde, celui de Megastoma, ou Scaphorhynchus, Swainson.

Il est certain que l'Amérique possède des groupes d'oiseaux dont les espèces ont une telle similitude de coloration, qu'au premier abord il est difficile de ne pas les confondre, et que ce n'est qu'après une scrupuleuse observation qu'on parvient à reconnaître les petites différences qui, se retrouvant néanmoins chez tous les individus d'une même localité, en constituent réellement des espèces distinctes, c'est ce que nous remarquons dans le groupe des Bécardes, dans celui des Picucules, et enfin dans celui des Tyrans, dont nous nous occupons maintenant. C'est ainsi qu'en observant dernièrement plusieurs Tyrans de Colombie, qu'au premier abord nous croyions être le Saurophagus sulphuratus, mais dont les ailes et la queue avaient plus de roux, nous avons fini, après une scrupuleuse comparaison, par reconnaitre entre elles des différences notables qui, jointes à la diversité des localités, ne nous ont plus laissé de doutes sur la distinction spécifique de cette espèce colombienne, dont voici la description :

"Saunophagus kufipennis, Lafr. - S. suprâ umbrinus, pileo nigro, cristato, cristâ in medio ranunculaceâ; vitıâ superci liari a fronte ad nucham pileum totum cingente, ailbâ; remigibus totis a basi rufis, tertiâ parte ap̧icali tantumimoủo nigro-fuscis, extùs rufo marginatis; rectricibus totis intùs rufis, extus nigrofuscis, rufo marginatis ; duabus mediis tantummodo intùs et extus fuscis rufo marginatis; subtùs uti apud Saurophagum sulphuratum totus citrinus, gulâ albâ. - Longit. tota, 22 cent. (ave 
arte farcto) ; alæ plicatæ, 12 cent. 172 ; caudæ; 9 cent. - Habitat in Colombiâ, Caracas. ")

Lorsqu'on rapproche cet oiseau du Saurophagus sulphuratus, on est frappé de leur grande analogie de formes et presque de coloration; cependant, notre rufipennis se distingue de l'espèco type par ses rémiges primaires entièreinent rousses jusqu'aux deux tiers de leur longueur; par les secondaires, qui le sont également sur toute leur partie interne et sur leur bord extérieur, n'ayant de noir que leur partie médiane le long de leurs tiges, et leurs grandes et moyennes couvertures étant marquées de même; il s'en distingue encore par ses rectrices rousses sur tout leur côté interne et sur leur bord externe, n'ayant de noirâtre qu'une bande longitudinale médiane le long de la tige. Le fond de la couleur supérieure est moins olive, et tire davantage sur la nuance terreuse. Quant à la forme, elle est pour ainsi dire la même; le rufipennis a cependant les pattes plus faibles, tout en ayant les ailes au moins aussi longues.

Les deux individus que nous possédons de cette espèce viennent de Caracas. L'espèce paraît se trouver dans toute la Colombie, et y remplacer le sulphuratus de Cayenne et du Brésil.

Nous possédons deux Saurophagus de Chuquisaca, en Bolivie, presque entièrement semblables au sulphuratus, quant à la coloration, mais sensiblement plus grands dans toutes leurs proportions : ainsi leurs ailes, ployées, ont plus de 15 centimètres de longueur, tandis que, chez le sulphuratus, elles n'en ont que 12. leur queue a 9 centimètres $1 / 2$; elle n'en a que $81 / 2 \mathrm{chez}$ le premier : de plus, la couleur jaune de leurs parties inférieures est d'un jaune plus blanchâtre; un de ces deux individus a nonseulement toutes les rémiges, sauf l'extrémité des primaires et leurs tectrices, bordées de roux, comme chez le sulphuratus, mais ses rectrices le sont également et réguliè- 
rement, et le dessus de sa tête est tout noir, sans apparence de jaune à la base des plumes.

Ces deux caractères nous paraissent indiquer le jeune âge chez cet individu. Nous pensons, du reste, que ces deux individus ne constituent pas, comme le rufipennis, une espèce distincte du sulphuratus, mais plutôt une variété de taille, ou race plus grande et particulière à la Bolivie.

Le second Tyran, dont nous avons reconnu la distinction spécifique, est le Scaphorhynchus Mexicanus, Nob.

"S. supra grisescente-olivaceus, remigibus primariis et secundariis nigro-fuscis, basi sultiliter rufescente marginatis, tertiariis palliclioribus; rectricibus fuscis, subtilissime et vix conspicuè rufescente fimbriatis; pileo capitisque lateribus nioris, fronte a grisescente, vittà latâ superciliari a fronte ad nucham extensâ allíi$d_{\star}$; verticis plumis basi pulchrè luteis, apice nigris; subtùs sulphiurascente flavus, gulà tołâ albidâ; rostrum nìgrum, ınagnum, elongatum, sed minus dilatatum quam in Scaphorhyncho pitangua, Gmelin (Bentaveo, Buff., enl. 212). - Longit. tota in exuviâ ave, 25 cent. ; alæ plicatæ, 13 cent.; caudæ, 9 cent. $1 / 2$; rostri a fronte, 3 cent; illius latitudo, 1 cent. 1/2. - Habitat Mexico. »

Cette espèce, qui offre les plus grands rapports ávec le Nei-nei d'Azara, ou Bentaveo de Buffon, col. 212, s'en distingue cependant par sa coloration supérieure d'un olive grisâtre, et non d'un olive brunâtre; par celle des parties inférieures, d'un jaune plus pâle ét plus teinté de soufré; par la forme de son bec, évidemment plus étroit, plus parallélogrammique; par sa taille plus grande, ses ailes ployées ayant 15 centinètres de longueur au lieu de 12; sa queue, $91 / 2$ au lieu de $81 / 2$, et néanmoins son bec n'étant pas plus long, mais étant bien plus étroit et moins dilaté.

Nous possédons un second individu entièrement semblable de forme et de coloration, et du Mexique comme 
lui, mais dont les plumes du vertex, au lieu d'être, comme les siennes, d'un beau jaune jonquille à leur base, sont d'un roux vif en cettc partie, offrant cependant vers l'occiput quelques plumes jaunes.

Nous possédons, en outre, quatre Scaphorhynchus, que nous regardons comme le Bentaveo de Buffon, dont trois entièrement semblables entre cux de forme et de colora. tion, et dont l'un a ses plumes verticales d'un beau jaune; l'autre les a rousses, tandis que le troisième les a presque toutes noires. La seule différence que présente ce dernier consiste dans un peu plus de brièveté et d'étroitesse du bec.

Le quatrième, qui nous parait évidemment un jeune, a la plus grande partie des plumes noirâtres du dos bordées de roussâtre; ses pennes alaires et caudales, et leurs tectrices supérieures sont aussi, dans tout leur pourtour, de la même teinte. Le noir de la coiffe est moins prononcé que chez les trois autres, et chaque plumè en est terminée de roussâtre sans qu'il y ait à leur base vestige de couleur jaune ou rousse, comme chez les deux premiers individus. Quant à la coloration des parties inférieures, elle est toutà-fait semblable.

Cette diversité de coloration à la base des plumes du vertex, chez plusieurs individus de ces deux espèces, qui d'ailleurs ne nous en ont présenté aucune autre dans le reste de leur plumage, nous a fait présumer fortement qu'elle est due à la différence de sexe et d'âge, et non à celle d'espèces, comme l'a cru Swainson, qui en a indiqué trois sous les noms de Hegastoma flaviceps, ruficeps et atriceps. Or, nous avons la certitude que les jeunes, encore revêtus de leurs premières plumes à bordures rousses, ont toujours la coiffe noire, avec les plumes terminées de roussâtre, comme nous l'avons remarqué chez notre quatrième individu du Scaphorhynchus pilangua, ce que nous avons encore reconnu chez un jeune du Surophagus sulphuralus, espèce si voisine. Nous ne nous sommes pas bor- 
né à l'observation des quatre individus de notre collection, nous l'avons étendue sur dix ou douze autres venant tant du Brésil que de Cayenne, et même de Colombie. Parmi tous ces exemplaires, dont les uns ont la base des plumes du vertex d'un jaune citron ou jonquille, les autres d'un roux canelle, nous n'avons pu trouver aucun autre caractère différentiel, et même chez les individus à coiffe toute noire en apparence, si on relève fortement les plumes du vertex et du front, on s'aperçoit que leur extrême base près du crâne est déjà colorée en jaune citron.

Que peut-on augurer de tout cela, si ce n'est que ces deux couleurs jonquille ou roux canelle, qui se mons trent indifféremment sur des individus à plumage d'ailleurs semblable, et paraissant le plumage d'adulte, ne peuvent indiquer que la différence de sexe, tandis que la coiffe, toute noire en apparence, de quelques-uns, mais dont l'extrême base est jaune citron, pouvait être, chez eux, la première livrée après celle du nid, livrée où l'oiseau n'aurait encore à l'extrême base de ses plumes frontales qu'une tache jaune citron qui, dans les mues suivantes, prendrait plus d'extension et une nuance plus franche ne présentant plus alors de noir qu'à l'extrémité des plumes.

Nous pensons donc que les deux espèces de Scaphorhynchus désignées par Swainson sous les noms de Megastoma flaviceps et ruficeps ne sont que les deux sexes d'une même espèce, probablement l'espèce commune et anciennement connue sous les noms de Pitangua-guacu, Marcgrave, Nei-nei, Azara, Bentaveo, Buffon, et Tyrannus carni . vorus, Vieillot, puisque nous trouvons cette distinction de couleurs chez des individus brésilièns et cayennais entièrement semblables d'ailleurs, et que nous l'avons remarquée également chez deux individus de notre Scaphorhynchus mexicanus, qui ne nous ont présenté aucune autre différence entre eux; et, quant à son Megastoma atriceps, 
nous sommes très-porté à croire que c'est encore le même oiseau à sa première livrée après celle du nid.

Du reste, cette opinion de ne voir, dans les individus à huppe jaune et ceux à huppe rousse, qu'une seule espèce, n'est pas nouvelle, car elle remonte à Marcgrave, qui, dans son Histoire dn Brésil, publiée en 1648, disait, en parlant de son Pilangua-guacu, "qu'entre ces oiseaux les uns avaient une tache orangée au sommet de la tête, les autres une jaune, "ce qui est cité par Buffon à l'article de son Bentaveo, pl. enl. 212.

On connaît depuis longtemps les deux figures des planches enluminées de Buffon désignées, l'une sous le nom de Bécarde à ventre jaune, $\mathrm{n}^{\circ} \mathbf{2 9 6}$, et l'autre sous celui de Geay à ventre jaune de Cayenne, $\mathrm{n}^{\circ} 249$. Latham et Gmelin ont également adopté, comme espèces distinctes, ces deux oiseaux, en leur donnant deux noms latins différents. Vieillot, au contraire, a pensé que c'était une erreur, et qu'ils représentaient une seule espèce, qu'il a eu tort de nommer Tyrannus magnanimus, puisque déjà elle en portait plusieurs, et entre autres celui de suiphuratus. Nous sommes entièrement de son avis, quant à l'identité de ces deux figures; nous pensons seulement que, de ces deux oiseaux de Cayenne, celui de la planche 296, sous le nom de Bécarde à ventre jaune, qui y est représenté et décrit avec le dessus de la tête tout noir, sans jaune au milieu, est le jeune, tandis que celui de la planche 249 , sous le nom de Geay à ventre jaune de Cayenne, et quỉ a une tache jaune sur le milieu de la tête, serait un mâle ou femelle adultes. C'est d'autant plus probable, qu'on n'a point reconnu depuis, à Cayenne, une seconde espèce aussi voisine de taille et de coloration du Saurophagus sulphuratus. 
MÉlanges ORNITHOLOGIQUes. - Sur une nouvelle espèce de Todier (Todus), par M. F. DE LAFRESNaYe.

Lorsque, dans la Revue de 1847, nous fîmes l'énumération des diverses espèces de Todiers connues jusqu'alors, et dont la plupart avaient été confondues sous le nom primitif de Todier vert (Todus viridis), dénomination qui n'apparticnt qu'à la seule espèce de la Jamaïque, fort distincte de toutes les autres, et la première qui ait été décrite sous ce nom par Browne, dans son Histoire de la Jamaïque en 1756; dans cette notice, disons-nous, et à l'article de notre Todus dominicensis, nous observions que le Todus subulatus de Gould, indiqué par cet auteur comme étant aussi de Saint-Domingue, et qui était entièrement conforme à notre dominicensis par sa taille, la largeur, la longueur et la denticulation de son bec, et sa coloration générale, n'en différant que par la pointe acuminée et non obtuse de ce bec, ne nous paraissait pas, d'après ce seul caractère, devoir constituer une espèce distincte, mais plutôt une variété, étant surtout propre à la même île.

Depuis lors, une nouvellc espèce, originaire aussi de Saint-Domingue, et distincte de ces deux-ci, en a été rapportée dernièrement avec le dominicensis, et nous a prouvé que, puisque cette île renfermait, à notre connaissance, deux espèces évidemment distinctes, le dominicensis et cette dernière, elle pouvait bien également en renfermer trois, et que le subulatus, qui en est aussi originaire, pouvait bien dès-lors, malgré sa grande analogie avec le dominicensis, constituer cette troisième espèce. Il résulterait donc de cette découverte récente qu'au lieu de quatre espèces que nous avions indiquées en 1847 , et qui viennent de l'être également, en 1850, par le prince de Canino, dans son Conspectus avium; il y en aurait réellement six de connues aujourd'hui, dont une, l'espèce type de la Jamaïque, trois de Saint-Domingue, une du Mexique, et une de Cuba et Porto-Rico. 
Nous allons décrire cette nouvelle espèce, renvoyant, pour les cinq autres, à notre article de la Revue, 1847, p. $\mathbf{5 2 6}$.

Todus angustirostris. - « T. supra totus prasino-viridis; subtùs albus, gulâ ut rite pulchrè rubro purpurinâ, utrinque vittâ mystacali albâ marginatâ, pectoris collique lateribus cinereis, hypochondriís vividè roseis; subcaudalibus anoque cilrinis; rostro debili, angusto, supra nigro, infia luteo-albescente. -Long. totâ in exuviâ ave, 10 cent.; rostri a fronte, 1 cent. 1 l2. - Habit. in Sancti-Dominicensis insulâ. ")

Cette petite espèce, la plus petite peut-être des six, égale à peine en grandeur le Todus multicolor. Elle s'en distingue au premier coup-d'oeil, ainsi que de toutes les autres, par l'étroitesse de son bec, noir en dessus, d'un blanc jaunâtre en dessous, obtus à sa pointe. Il n'est point finement denticulé sur ses bords, comme chez le dominicensis et le subulatus; et, en cela, il se rapproche du viridis, du multicolor et du mexicanus. - Il a toutes ses parties supérieures du même vert que ses cinq congénères. - La partie gulaire est également d'un beau rouge pourpré, avec l'extrémité des plumes frangée de blanc soyeux; mais cette sorte de hausse-col est plus large chez lui que chez le viridis et le mullicolor, et égal à celui du dominicensis et du subulalus. Son bec, qui n'est pas plus long que celui du multicolor; est évidemment plus étroit el plus parallélogrammique, et en diffère encore par la couleur noire de sa mandibule supérieure. Une bande étroite blanche sépare également, de chaque côté, le rouge de la gorge du vert des côtés de la tête, et est terminée, au-dessous des oreilles, par une tache grise, au lieu de l'être par une bleue, comme chez le multicolor, et le haut de la poitrine, audessus de la bande cendrée pectorale, est d'un blanc pur, comme tout le ventre, depuis cette bande jusqu'à l'anus. Ce blanc n'est point teinté de rosâtre ni d'aucune autre nuance; les côtés de la poitrine seulement sont d'un gris 
[From the Proceedings of the Zoological Society of London.]

1. A Monograph of the genus Ruticilla, with Descrip-

TIONS OF SOME NEW SPECIES.

By Frederic Moore, Assist. Mus. East India House.

Genus Ruticilla (Ray), Brehm.

Syn. Ficedula, Boie. Phoenicura, Swains. Chomarrhornis*, Hodgs.

1. Ruticilla phenicura, linn.

Syn. Motacilla phonicurus, Linn. S. N. i. p. 335.

Sylvia phoenicurus, Lath. Ind. Orn. ii. p. 511 ; Gen. Hist. vii. p. 21. Ruticilla sylvestris, Brehm, Vœg. Deutschl. p. 363. t. 21. f. 4.

Phøenicura ruticilla, Swains. Class. of B. ii. p. 240. Gould, Birds of Eur. t. 95.

Ficedula phoenicura, Boie, Isis (1822), p. 553.

Phonicura muraria, Swains. Faun. Bor. Amer. ii. p. 489.

Ruticilla phoenicurus, Bonap. Geogr. Comp. List of B. p. 15, et C. G. Av. p. 296 . G. R. Gray, Gen. of B. i. p. 180. Blyth, Journ.

A. S. Beng. xvi. p. 133 ; Catal. B. Mus. A. S. Beng. p. 168.

? Phoenicura albifrons, Brandt.

The Redstart, Bewick.

Hab. Europe; W. Asia ; N. India. In Mus. East India Company.

2. Ruticilla phenicuroïdes, nobis.

Forehead, lores, ear-coverts, throat and breast black, extending much lower down than in phonicura; crown, neck, back and upper wing-coverts ash, with a rufous tint, the ash palest on the crown; wings brown, with the edges of the exterior webs paler; from breast to vent, under wing-coverts, upper and lower tail-coverts and tail (except the two medial feathers which are brown) rufous red. Male.

Length, 5 inches ; wing, 3 , the first primary being a $\frac{1}{4}$ inch longer, and the second a $\frac{1}{4}$ inch shorter than in phoenicura; tail, $2 \frac{1}{2}$; bill to gape, $\frac{7}{10}$, more elongated, not so broad at base, and it is also a trifle longer; and tarse, $\frac{6}{8}$.

Hab. N. India. In Mus. East India Company.

This species is closely allied to $R$. phonicura, but is readily distinguished from it in the absence of the white on the front of the crown.

* Chaimarrornis, Hodgs., corrig. Agassiz. 
3. Mutichlat tithys, Scopoli.

Syn. Sylvia tithys, Scopoli, Ann. i.p. 157. Lath. Ind. Orn. ii. p. 512 ; Gen. Hist. vii. p. 23.

Ruticilla tithys, Brehm, Vog. Deutschl. p. 365. Gould, Birds of Eur. t. 96. G. R. Gray, Gen. of Birds, i. p. 180, et Cat. Brit. B. in B. M. p. 62. Bonap. C. G. Av. p. 296.

? Motacilla evythacus, Linn. S. N. i. p. 335.

? Motacilla gibraltariensis, Gmel. S. N. L. i. p. 987.

? Motacilla atrata, Gmel. S. N. I i. p. 988. Lath. Ind. Orn. ii. p. 514 (nee Lath. Gen. Hist.).

The Black Redstart, Eyton.

Hab. Europe; W. Asia; N. Africa.

\section{Ruticilla nipalinsis, Hodgson.}

Syn. Phonicura nipalensis, v. atrata, Hodgs. Gray's Zool. Misc. (1844) p. 83.

Sylvia atrata, Lath. Gen. Hist. vii. p. 26 (nec Gmel. et Lath. Ind. Orn.).

Phonicura atrata, Jard. et Selby, Ill. Ind. Orn. t. 86. f. 3. Sykes, P. Z. S. (1832) p. 92. Jerd. Madras Journ. x. p. 267. Blyth, Journ. A. S. Beng. xi. p. 190.

Ruticilla atrata, G. $\mathbb{R}$. Graj, Gen. of Birds, i. p. 180. Hodgs. Cat. B. of Nep. p. 68.

Ruticilla indica, Blyth, Catal. B. Mus. A. S. Beng. p. 168 (1850). Bonap. C. G. Av. p. 296.

Black Indian Redstart, Jard. et Selby.

Thirt-hira (i. e. Shaker'), Hind., Jerdon.

Thirthir Kumpa, Plains, Royle.

Phiriva or Lülyŭrdi, Beng., Dr. F. (Buch.) Hamilton, MS. ii. p. 94.

Mal. India generally. In Mus. East India Company.

"This species is very common in most parts of India during the cold weather, but more so in the table-land, I think, than in the Carnatic. It is solitary, frequenting wooded places, gardens, bedges, old walls and out-buildings, being often seen about the roofs of houses. It feeds on the ground, on wasps, ants, and various other insects. Iias a most peculiar quivering motion of its tail, especially after f'eeding." Jerdon.

5. Rutucilla Hodgsoni, nobis.

Syn. Phanicura ruticilla, Hodgs. Gray's Zool. Misc. (1844) p. 82 (nec Swains.).

Ruticilla Reevesii, Hodgs. Cat. Birds of Nepal, p. 67 (nec J. E. (sar).

Plucenicura Reevesii, Blyth, Journ. A. S. Beng. xii. p. 963 (nec J. E. (rray).

Ruticille erythrogastra, Blyth, Cat. B. Mus. A. S. Beng. p. 168 (nec fiuldenst.).

Thareapmi, Nepal, Hodgson.

Ilal. Nepal. In Mus. East India Company. 
Male : forehead, lores, ear-coverts, throat and breast black ; fore part of crown clear white (much narrower than in phonicura); hind part of crown, neck, back and upper wing-coverts fine ash, lightest on the crown; wings above dusky brown, the exterior margin of the basal half of the secondaries white, forming a patch ; from the breast to vent, under wing-coverts, rump, upper and lower tail-coverts and tail (except the interior and exterior margins only of the two middle feathers which are dusky brown) rufous red. The female may be readily distinguished by a general puffy appearance, and by the relative length of wing, \&c.

Length, 6 inches ; wing, $3 \frac{3}{8}$; tail, $2 \frac{6}{8}$; bill to gape, $\frac{6}{8}$; and tarse, $\frac{7}{8}$.

\section{Ruticilla rufogularis, nobis.}

Male : crown and back of neck mixed grey and ash; lores, earcoverts and sides of neck black; wings and medial tail-feathers dark brown; apical margin of the exterior web of the outer tail-feather dusky; smaller wing-coverts (except the feathers immediately on the shoulder), scapulars, basal portion of the speculars, and apical margins of the greater wing-coverts white; exterior margin of the secondaries pale rufescent; throat and breast, back and upper tailcoverts and tail rufous; abdomen, under wing- and tail-coverts pale rufescent. Female : cinereous brown above, rufescent beneath ; wings dark brown, margined with pale rufescent; lower part of back, upper tail-coverts and tail rufous, the two medial feathers dark brown, exterior margin of the outer dusky.

Hab. N. India. In Mus. East India Company.

\section{Ruticilla erythrogastra, Güldenstadt.}

Syn. Motacilla erythrogastra, Güldenst. Nov. Comm. Petrop. xix. p. 469 . t. 16, 17. Gmel. S. N. L. i. p. 975.

Sylvia erythrogastra, Lath.Ind.Orn.ii. p.503; Gen. Hist.vii.p. 27. Motacilla ceraunia, Pallas, Zoogr. i. p. 478.

Ruticilla aurorea, G. R. Gray, Gen. of Birds, i. p. 180 (nec Pallas).

Ruticilla grandis, Gould, P. Z. S. (1849) p. 112.

Ruticilla erythrogastra, Bonap. C. G. Av. p. 296. Gould, Birds of Asia, t. 50.

The Chestnut-bellied Warbler, Lath.

Hab. Cashmere; Kumaon; Nepal. In Mus. East India Company.

"This species frequents the gravelly hollows of the Caucasian torrents during the whole of the summer, and migrates southward, in search of food, on the approach of winter ; it runs along the banks of rivers ; is restless, but not fearful ; often moving its tail while sitting on the low shrubs; it makes its nest among the branches of the sea buckthorn, of the berries of which it is very fond."'-Güldenstadt.

\section{Ruticilia Vigorsi, nobis.}

Female : above, head, neck and back cinereous; wings dusky, witl paler margins; beneath rufescent; upper tail-coverts and tail rufous, 
the two medial feather's dusky, as is also the exterior web of the outer and tip of each feather, but paler ; bill and legs black.

Length, 7 inches; wing, $4 \frac{1}{4}$; first quill $1 \frac{1}{2}$ inch shorter than the second, the third $\frac{6}{10}$ longer than the second, fourth and fifth equal and longest, the third and sixth equal and but a trifle shorter than the fourth and fifth, the seventh a $\frac{1}{4}$ inch longer than the second, the eighth $\frac{1}{8}$ shortel than the second; tail, 3 ; bill to gape, $\frac{6}{8}$; and tarse, 1.

Hab. N. India. In Mus. East India Company.

This may eventually prove to be the female of a species allied to $R$. erythrogastra, but having no white wing-patch.

9. Ruticilifa aurorea, Pallas.

Syn. Motacilla aurorea, Pall. Zoogr. i. p. 477. Kittl. Kupf. Vœg. t. 26. f. 1. Gmel. S. N. I. i. p. 976. Lath. Hist. rii. p. 92.

Phonicura Reevesii, J. E. Gray, Zool. Misc. (1832) p. 1. M'Clelland, P. Z. S. 1839, p. 161.

Ruticilla aurorea, G. R. Gray, Gen. of Birds, i. p. 180 (excl. syn.). Bonap. C. G. Av. p. 296.

Lusciola aurorea, Schlegel, Faun. Japon. t. 21 D.

Daurian Warbler, Lath.

Reeves's Redstart, Gray.

Hab. N. India; China; Japan. In Mus. East India Company.

Male : forehead, lores, ear-coverts, throat, fore part of breast, back, upper wing-coverts, apical and basal portion of the secondaries and tertiaries, and the two medial tail-feathers black; exterior margin of the outer tail-feather and apical margin of the rest dusky black; medial portion of both webs of the secondaries and tertiaries white; crown of head and back of neck slaty ash, rather whitish above the ear-coverts; breast, abdomen, under wing-coverts, upper and lower tail-coverts and tail (except as above) rufous. Female : above brown, the wing-patch rufescent white; beneath rufescent; upper and lower tail-coverts and tail (except the medial feathers of the latter which are dusky brown) rufous; bill and legs black.

Length, 5 inches; wing, 3 ; tail, $2 \frac{1}{2}$; bill to gape, $\frac{5}{8}$; and tarse, $\frac{3}{4}$.

\section{Ruticilia ieucoptera, Blyth.}

Syn. Phrenicura leucoptera, Blyth, Journ. A. S. Beng. xii. p. 962.

Tiuticilla leucoptera, Blyth, Journ. A. S. Beng. xvi. p. 134 ; Catal. B. Mus. A. S. Beng. p. 168. G. R. Gray, Gen. of Birds, i. p. 180. Bonap. C. G. Av. p. 296.

Mal,. Malacea; Java. In Mus. As. Soc. Bengal.

"Size of $R$. phrenicura, and much resembling in plumage $R$. nipalensis, but smaller and the wings much shorter than in the latter species; it is also generally similar to $R$. phoenicura, but has no white on the forehcad, which, with the crown, neck, and fore part of the back, are ash-grey; the midule of the back is black, as are also the lores, ear-coverts, throat and breast; and the rest of the under parts, with the lump and tail, except the medial feathers of the latter, are 
bright ferruginous, the exterior web of the outermost tail-feather being marked with dusky; wings dusky, having a large white patch occupying the base of the secondaries and tertiaries, extending over both webs of each feather; bill and feet black. According to season, the dorsal plumage is margined with brown edgings, the under parts more slightly with greyish, and the wing-coverts with brown. Female plain brown above, paler beneath, with rufous tail, and the same great white wing-patch as in the male.

"Length about 6 inches ; of wing, $2 \frac{3}{4}$; tail, $2 \frac{3}{8}$; bill to gape, $\frac{5}{8}$; tarse, $\frac{3}{4}$."

\section{Ruticilla Caruleocephala, Vigors.}

Syn. Phœnicura caruleocephala, Vigors, P. Z. S. 1830, p. 35. Gould, Cent. of Birds, t. 25. f. 2. Blyth, Journ. A. S. Beng. xi. p.190.

Ruticilla caruleocephala, G. R. Gray, Gen. of Birds, i. p. 180. Hodgs. Catal. B. of Nep. p. 68. Blyth, Journ. A. S. Beng. xvi. p.134; Catal. B. Mus. A. S. Beng. p. 168. Bonap. C. G. Av. p. 296.

Hab. N. India. In Mus. East India Company.

\section{Ruticilla schisticeps, Hodgson.}

Syn. Phonicura schisticeps, Hodgs. Gray's Zool. Misc.(1844)p.83.

Ruticilla schisticeps, Hodgs. Catal. B. of Nep. p.69, etApp. p.153.

Hab. Nepal (No.813. Hodgs. Catal.).

"Side of the head and neck, back, wings and tail black; top of the head pale slaty blue ; throat and large patch on each wing white; lower part of breast and abdomen rufous chestnut.

"Length, 6 inches; wing, 3 inches 4 lines; bill from gape, 7 lines; tarse, $10 \frac{1}{2}$ lines."

The only known example of this species is in the British Museum.

\section{Ruticilla Nigrogularis, nobis.}

Crown of the head slaty blue, lightest on the forehead ; lores, earcoverts, throat, back of neck, back, upper wing-coverts, two middle tail-feathers entirely, and the rest (except the basal portion) black; wings blackish brown, the scapulars, outer edges of the secondaries and under wing-coverts white ; breast, flanks, belly, rump, upper and lower tail-coverts and base of tail (except the two middle feathers) bright chestnut; the vent and under tail-coverts have a few white feathers intermixed; bill and legs black.

Length, 6 inches ; wing, $3 \frac{3}{8}$; tail, $2 \frac{6}{8}$; bill to gape, $\frac{5 \frac{1}{8}}{8}$; tarse, $\frac{5}{8}$.

Hab. Nepal (No. 813*. Hodgs. Catal.). In Mus. East India Company.

This species is closely allied to schisticeps, but differs in having the throat black instead of white.

\section{Ruticilla frontalis, Vigors.}

Syn. Phœnicura frontalis, Vigors, P. Z. S. 1831, p. 172. Gould, Cent. of Birds, t. 26. f. 1. Blyth, Journ. A. S. Beng. xi. p. 190.

Ruticilla frontalis, G. R. Gray, Gen. of Birds, i. p. 180. Hodgs. 
Cat. B. of Nep. p. 68. Blyth, Journ. A. S. Beng. xvi. p. 134; Catal. B. Mus. A. S. Beng. p. 168. Bonap. C. G. Av. p. 296. Ruticilla melanura, Less. Rev. Zool. (1840) p. 265.

Phcenicura tricolor, Hodgs. Gray's Zool. Misc. (1844) p. 83. Hab. N. India. In Mus. East India House.

15. Ruticilla Fuliginosa, Vigors.

Syn. Phonicura fuliginosa, Vigors, P. Z. S. 1830, p. 35. Blyth, Journ. A. S. Beng. xi. p. 190.

Ruticilla fuliginosa, G. R. Gray, Gen. of Birds, i. p. 180. Hodgs. Cat. B. of Nep. p. 68. Blyth, Journ. A. S. Beng. xvi. p. 134; Cat. B. Mus. A. S. Beng. p. 169. Bonap. C. G. Av. p. 296.

Phonicura plumbea, Gould, P. Z. S. 1835, p. 185.

Ruticilla simplex, Less. Rev. Zool. (1840) p. 265.

Phonicura rubricauda, Hodgs. Gray's Zool. Misc. (1844) p. 82 (the male).

Phonicura lineoventris, Hodgs. MS. (the female).

Hab. N. India. In Mus. East India Company.

"Found along rivers, near or among mountains, haunts rocks just appearing above the torrent. Feeds on coleopterous insects. Continually spreading its tail out like a fan, and in a vibrating manner." - Griffith.

16. Ruticilla leucocephata, Vigors.

Syn. Phoenicura leucocephala, Vigors, P. Z. S. 1830, p. 35. Gould, Cent. of Birds, t. 26. f. 1.

Sylvia erythrogastra, var. A, Lath. Hist. vii. p. 28.

Ruticilla leucocephala, Less. Rev. Zool. (1840) p. 265 . G. R. Gray, Gen. of Birds, i. p. 180. Hlodgs. Cat. B. of Nep. p. 68. Blyth, Journ. A. S. Beng. xvi. p. 134 ; Catal. B. Mus. A. S. Beng. p. 169. Bonap. C. G. Av. p. 296.

Chaemarrhornis leucocephalus, Hodgs. Gray's Zool. Misc. (1844) p. 82.

The White-capped Redstart.

Gir-Chaondeea, Hind., IIardwicke.

Kalee pholia, Mohun Ghats, Royle.

Hal. N. India.

"This species is extremely common in the valley of the Dhoon. and also in the hills, along the banks of streams and rivers, flitting from rock to rock and stone to stone, and eternally shaking and spreading its tail."-IItutton.

17. Phenicura eryturonota, Eversman.

Syn. Sylvia spylhronota, Eversm. Addend. Pallas, Zoogr. Fasc. ii. Tiuticilla enythronota, G. R. Gray, Gen. of Birds, i. p.180. Bonap. C. G. Av. p. 297.

Ilab. Caucasus (non vidi). 
2. Descriptions of SOME NeW and LitTle-known SPECies of Birds from Northern India, contained in the Museum of the Hon. East India Company. By Frederic Moore, Assist. Hon. Comp. Mus.

Fam. Merulide, Vigors.

Subfam. Myiotherina, Swains.

Genus Pnö̈ PYGA, Hodgs.

Syn. Microura, Gould (nec Ehrenb.).

\section{Pnö̈ pyga longicaudata, nobis.}

Colour of the upper parts deep olive-brown, the feathers slightly margined with black, and having blackish shafts; wings and tail inclined to ferruginous brown; upper tail-coverts long; throat whitish ; breast and sides of abdomen pale dusky ferruginous, the feathers having pale centres and blackish margins; centre of lower part of breast and abdomen white; flanks the same as the back; vent and under tail-coverts dark ferruginous; bill dark horn, legs paler.

Length, $4 \frac{1}{2}$ inches; of wing, 2 ; the tail is rounded, the central feathers being $2 \frac{1}{4}$ inches long, and the outer $1 \frac{1}{2}$ inch; bill to front, through the feathers, $\frac{1}{2}$ inch ; to gape, $\frac{6}{10}$, and tarse, $\frac{8}{10}$.

$H a b$. N. India.

\section{Genus Brachy pteryx, Horsfield, p. s. d.}

2. Brachypteryx nipalensis, Hodgson.

Male : colour above a greyish cyaneous, or light greyish blue, as are also the cheeks, sides of breast and flanks, and paling to greyish white on the centre of the breast; throat, middle of belly, vent and under tail-coverts, streak over but not extending beyond the eye, white. Female: ferruginous brown above, paling on the sides; throat, breast, belly, under tail-coverts and streak over the eye, whitish, the feathers on the breast and sides fringed with brown; bill dark horn, legs paler.

Length, $4 \frac{1}{4}$ inches; of wing, $2 \frac{1}{2}$; tail, $1 \frac{3}{8}$; bill to gape, $\frac{6}{8}$; tarse, 1 . Hab. Nepal (No. 943, Hodgs. Catal.).

This species is allied to Br. cruralis, Blyth, but may readily be distinguished by its smaller size, shorter tail, paler colour of the upper parts, and by its white throat and belly. 
Genus Callene, Blyth, Journ. A. S. Beng.p. 136 (1847).

Syn. Cinclidium, Blyth (nec Gould).

Gen. Char. "Bill shorter than the head, straight, slender, higher than broad, the ridge of the upper mandible tolerably acute, and its tip rery slightly enarginated; inferior gonys ascending for the terminal half, imparting to the bill the appearance of a tendency to bend upward: naral apertures an elongate-oval fissure in the lateral nasal membrane, and partially impended by the short semi-reflected frontal feathers; gape armed with a few small setæ; wings and tail rounded, the 4 th, 5 th and 6 th primaries equal and longest ; legs and toes slender, the tarsi smooth and unscutellate, and very long, as is also the middle toe; claws but moderately curved, and of little more than mean length; plumage light, soft and full, having a scale-like appearance on the crown, breast and belly."

3. Callene frontale, Blyth.

Syn. Cinclidium frontale, Blyth, Journ. A. S. Beng. xi. p. 181, xii. p. 954. t. p. 1010 . Bonap. C. G. Av. p. 301.

Callene frontale, Blyth, Journ. A. S. Beng. xvi.p. 136 ; Catal. B. Mus. A. S. Beng. p. 178.

Ruticilla frontalis, G. R. Gray, Gen. of Birds, i. p. 180.

Brachypteryx scapularis, Horsf. MS.

Hab. Nepal (No. 950, Hodgs. Catal.).

Spec. Char. "Plumage dark fusco-cyaneous, the rump dusky; flanks somewhat ashy, and middle of the belly slightly grey-edged; lores and immediately above the beak blackish, contrasting with a bright cærulean forehead; bend of the wing also cærulean, but less bright; and winglet, primaries and their coverts, secondaries and tertiaries, dark olive-brown; a white spot on the under surface of the wing, beneath the winglet; bill black; legs dusky brown.

"Length, $7 \frac{5}{8}$ inches; of wing from bend, $3 \frac{3}{8}$; middle tail-feathers, $3 \frac{1}{2}$, the outermost $\frac{3}{4}$ of an inch shorter' bill to forehead nearly $\frac{5}{8}$, to gape $\frac{13}{16}$; tarse, $1 \frac{3}{8}$; middle toe and claw, $1 \frac{3}{16}$; hind toe and claw, $\frac{3}{4}$; the last, $\frac{3}{8}$.

"This bird is reported to be a fine songster, and heard chiefly in the evening." - Blyth.

Subfam. Trmalina.

Genus Trichastoma, Blyth.

Syn. Malacocincla, Blyth.

Gien. Char. "Bill as long as the head, rather stout, high, much compressed, the tip of the upper mandible pretty strongly hooked, but indistinctly emarginated, and its ridge obtusely angulated towards the base, the remainder scarcely angulated; gape but little widened, and fecbly bristled; nostrils large and subovate, with oval aperture to the froint, a little removed from the base of the bill; tarse of mean 
length and strength, as long as the middle toe with its claw; the claws suited for perching, compressed, and moderately curved, that of the hind toe rather large ; wings moderate, with the first primary reaching to about their middle, the second much shorter than the third, and the fourth longest; tail rather short, weak and even, except that its outermost feathers are a little shorter than the rest; plumage full and lax, the coronal feathers somewhat elongated and of a spatulate form."

4. Trichastoma Aввотti, Blyth.

Syn. Malacocincla Abbotti, Blyth, Journ. A. S. Beng. xiv. p. 601 (1845).

Trichastoma Abbotti, Blyth, J. A. S. Beng. xvi. p. 462 ; Catal. B. Mus. A. S. Beng. p. 147. Bonap. C. G. Av. p. 259.

Malacopteron Abbotti, G. R. Gray, Gen. of Birds, iii. App. p. 9. Hab. Arracan (No. 919, Hodgs. Catal.).

Spec. Char. "Colour above plain olive-brown, tinged with rufous on the rump and tail; the upper tail-coverts ferruginous brown, under parts paler; the throat and middle of the belly white; the earcoverts, sides of the breast and flanks rufescent ; the lower tail-coverts weak ferruginous; bill horn-colour, paler beneath.

"Length, 6 inches ; of wing, 3 ; tail, $2 \frac{1}{8}$; bill to gape, $\frac{11}{12}$; and tarse the same."-Blyth.

Mr. Blyth states Arracan to be the habitat of this bird. Specimens have lately been presented to the Company's Museum by B. $\mathbf{H}$. Hodgson, Esq., from Nepal, and by Capt. R. C. Tytler from Dacca.

Fam. Sylviade, Vigors.

Subfam. Saxicolina, Vigors.

Genus Nemura, Hodgson.

Syn. Ianthia, Blyth.

5. Nemura Hodgsoni, nobis.

Male : colour of the upper parts purplish blue, brightening to vivid ultramarine on the crown; forehead, lores and sides of head, wings and tail black, the two latter margined with blue externally; on the rump some of the feathers are tipped with white; upper tail-coverts blue; lower parts yellowish ferruginous; under wing-coverts, flanks and under tail-coverts white. Female : ferruginous brown, richer on the rump; wings and tail dusky, margined with ferruginous brown externally; lores, ear-coverts and under parts pale dusky ferruginous; under tail-coverts white; bill black; legs pale horn.

Length, $3 \frac{3}{8}$ ths of an inch; wing, $3 \frac{7}{8} ;$ tail, $1 \frac{1}{4}$; bill to front, $\frac{2}{8}$; to gape, $\frac{3 \frac{1}{2}}{8}$; tarse, $\frac{I}{2}$ an inch.

Hab. Nepal (No. 949, Hodgs. Catal.). 


\section{Subfam. Philomelina, Swains.}

\section{Genus Tarsiger, Hodgson.}

\section{Tarsiger superciliaris, Hodgson.}

Nale : above deep cyaneous; wings brown, margined with cyaneous and rufous on the shoulder, primaries also margined with rufous, secondaries with cyaneous; tail brown, margined with cyaneous; a white superciliary streak extending one inch and a half from the nares to the back of the neck; lores and cheeks black; beneath rufous; centre of belly white. Female : olive-brown above; wings and tail brown, margined with rusty olive; superciliary streak not so clear as in the male; beneath pale dull rufous; centre of belly and shafts of the underside of tail white; bill black; legs horn colour.

Length, $5 \frac{1}{2}$ inches; of wing, $3 \frac{2}{8}$; tail, $2 \frac{25}{8}$; bill to gape, $\frac{5}{8}$; tarse, $1 \frac{1}{8}$. Hab. Nepal (No. 924, Hodys. Catal.).

\section{Subfam. Sylviana, Vigors.}

\section{Genus Prinia, Horsfield, p. s. d.}

\section{Prinia cinereocapilla, Hodgson.}

Crown of the head grey, the shaft and margin of the feathers being darker; nareal and frontal phumes, a streak over and beyond the eye, and the whole under parts rufescent, brightest on the flanks and thighs; lores and upper part of ear-coverts greyish; nape, back, rump and wings bright rufous brown, the tips of the latter dusky; tail the same colour as the back, paler beneath, with a terminal dusky band and rufescent white tips; bill black; legs pale horn.

Length, $4 \frac{1}{4}$ inches; wing, $1 \frac{8}{12}$; tail, 2 ; bill to front, $\frac{3}{8}$; to gape, $\frac{5}{8}$; tarsus, $\frac{3}{4}$; middle toe and claw, $\frac{7}{12}$; hind ditto, $\frac{6}{12}$.

Hab. Nepal (No. 890*, Hodgs. Catal.).

\section{Genus Suya, Hodgson.}

\section{Suya atrogularis, nobis.}

Colour above dusky brown, in some specimens ashy brown; edge of wing at shoulder and under wing-coverts buff; primaries margined with buffish and secondaries with rufescent brown; a whitish streak extending from the base of the lower mandible to the end and under the ear-covert; chin, throat, side of neck, breast and centre of abdomen black, the feathers of the two latter broadly centred with white; flanks and sides of abdomen mixed grey, brown and rufescent; rent buff; thighs buffy rufous; tail paler than the back, and has no perceptible terminal band; bill horn, feet paler.

Length, $6 \frac{1}{2}$ inches, including the tail ; wings, from $1 \frac{3}{4}$ to 2 inches in some specimens; central feathers of tail from $3 \frac{1}{2}$ to 4 , its outermost being only 1 inch; bill to gape, $\frac{8}{12}$; to front, $\frac{5}{12}$; height from chin to front, $\frac{1}{10}$; width at chin, $\frac{2}{10}$; tarsi, $\frac{7}{8}$.

Hab. Darjecling; Nepal (No. 893, Itodys. Catal.). 
A single specimen of this beautiful bird has been in the Museum of the East India Company for a number of years, collected in Darjeeling by F. T. Pearson, Esq. ; and B. H. Hodgson, Esq., has brought home several examples from Nepal.

Genus Drymorca, Swains. p. s.d.

9. Drymoica nipalensis, Hodgson.

Above rufous brown; wings dusky brown, the primaries exteriorly and secondaries interiorly and exteriorly margined with bright rufous brown; tail rufous brown, paler beneath, and distinctly rayed, has a terminal dusky band, the tips being pale but not white; the whole under parts rufescent, as in Prinia socialis, Sykes; bill dusky horn, paler below at base; legs pale horn.

Length, $4 \frac{\mathrm{I}}{2}$ to 5 inches; wing, $1 \frac{10}{12}$; tail varying from $2 \frac{1}{2}$ to 3 ; bill to front, $\frac{4}{12}$; to gape, $\frac{7}{12} ; \operatorname{tarsi}, \frac{10}{12} ;$ middle toe and claw, $\frac{8}{12}$; hind ditto, $\frac{7}{12}$.

Hab. Nepal (No. 913, Hodgs. Catal.). 
3. Notice of the species of the Genus Orthotomus of HoRsfield, WITH DEsCRIPTIONS OF A NEW SpECIES, AND of those hitherto known. By Frederic Moore, Assist. Mus. East Inda Company.

Fam. Sylviade, Vigors.

Subfam. Sylviana, Vigors.

Genus Orthotomus, Horsfield.

Syn. Ealela, Lesson.-Sutoria, Nicholson.

1. Orthotonus sepium, Horsfield.

Syn. Orthotomus sepium, Horsf. Trans. Limn. Soc. xiii. p. 166 (1820). Lath. Hist. iv. p. 265. Temm. Pl. Col. 599. f. 1. G. R. Gray, Gen. of Birds, i. p. 162. Blyth, Catal. B. Mus. A. S. Beng. p. 145. Bonap. C. G. Av. p. 282.

The Chiglet Creeper, Lath.

Chiglet of the Javanese, Horsf.

Hab. Java.

O. sepium.-The forehcad, lores, over the eyes and ear-coverts, ear-coverts themselves, base of lower mandible and chin ferruginous, palest on the sides and chin; top of head, back and tail brownish olive, having a greenish tinge; wings dusky, broadly margined with brownish olive; throat and breast asiny black, the rest of the under parts yellowish; tail with a terminal dusky band, tipped with yellowish; thighs ferruginous; bill brownish, paler below; legs pale.

Length, $4 \frac{1}{2}$ inches; of wing, $1 \frac{8}{10}$; tail, $1 \frac{3}{4}$; bill to gape, $\frac{7}{10}$; tarsus, $\frac{3}{4}$.

The above description is taken from Dr. Horsficld's typical specimens, contained in the Mus. East India Company.

2. Orthotomus atrogularis, Temminck.

Syn. Orthotomus atroyularis, Temm. Text de Pl. Col.599 (1836). G. R. Gray, Gen. of Birds, i. p. 162. Bonap. C. G. Av. p. 282.

Ila\}. Malacea; Borneo.

O. atrogularis.- "The forehead, top of the head and occiput bright brownish red; the neck, the back and the wings of a grassy green; the tail of a yellowish green, marked near the end and on the inner web with a narrow yellowish band; the chin, throat, breast and upper parts of flanks pure black, the sides of the abdomen yellowish; middle of the belly and abdomen white; bill and feet brown. No difference in the sexes.

"Length, $3 \frac{1}{2}$ inches." - Termm. 
3. Orthotomus flavoviridis, nobis.

The forehead, crown, round the eyes, and occiput ferruginous; back and rump yellowish green; tail more dusky green; wings brown, broadly margined exteriorly throughout with yellowish green ; chin, base of lower mandible, ear-coverts, centre of some of the feathers of the throat and breast white; lower part of the breast ash and white ; centre of abdomen white; throat and fore part of the breast black, centred as above; flanks yellowish, extreme edge of shoulder of wing yellow; under part of tail yellowish, with a terminal dusky band, tipped with yellowish; thighs greenish ferruginous. Specimens labeled "male."

Length, 4 inches; of wing, $1 \frac{3}{4}$; tail, $1 \frac{1}{2}$; bill to gape, $\frac{7}{10}$; tarsus, $\frac{7}{10}$. Hab. Malacca. In Mus. East India Company.

4. Orthotomus edela, Temminck.

Syn. Orthotomus edela, Temm. Pl. Col. 599. f. 2 (1836). G. R. Gray, Gen. of Birds, i. p. 162. Blyth, Catal. B. Mus. A. S. Beng. p. 144. Bonap. C. G. Av. p. 282.

Motacilla sepium, Raffles, Trans. Linn. Soc. xiii. p. 313. Lath. Hist. vii. p. 218 (nec Horsf. v. Lafres.).

Edela ruficeps, Less. Cent. Zool. p. 212. t. 71 (1834) (nec Less. Tr. d'Orn.).

Kachichi of the Malays of Sumatra, Raffles.

Hab. Malayan peninsula; Sumatra. In Mus. East India Company.

O. edela.— "The forehead, lores and crown ferruginous; the entire lower parts whitish, but the sides of the neck slightly variegated with clear ashy; back of neck, back, wing-coverts and tail greenish; the wings ashy, but bordered with greenish ferruginous; the tail is regularly of one colour; the bill and feet brownish."'-Temm.

Length, $4 \frac{1}{4}$ inches; of wing, $1 \frac{3}{4}$; tail, $1 \frac{1}{2}$; bill to gape, $\frac{3}{4}$; tarsus, $\frac{5 \frac{1}{2}}{8}$.

\section{Orthotomus Ruficeps, Lesson.}

Syn. Edela ruficeps, Less. Tr. d'Ornith. p. 309 (1831) (nec Less. Cent. Zool.).

Orthotomus sericeus, 'Temm. Text de Pl. Col. 599 (1836). G. R. Gray, Gen. of Birds, i. p. 162. Bonap. C. G. Av. p. 282.

Hab. Malacca; Borneo.

O. ruficeps. - The forehead, crown, occiput, lores and upper part of ear-coverts bright ferruginous; the chin, base of lower mandible, lower part of ear-coverts and the rest of the under parts silky white; back, rump and wings deep ash ; tail bright ferruginous, at base ashy, and with no terminal band; thighs ferruginous; bill yellowish brown, pale below ; legs pale.

Length, $4 \frac{1}{2}$ inches; wing, 2 ; tail, $1 \frac{3}{4}$; bill to gape, $\frac{7}{8}$; tarsus, $\frac{8}{10}$.

The above description is from a specimen in the East India Company's Museum. This species may readily be distinguished by the 
bright ferruginous colour of the head, the tail also being of the same colour, and in the bill being considerably longer and stouter (though strictly typical) than in any of the other known species.

\section{Orthotomus cineraceus, Blyth.}

Syn. Orthotomus cineraceus, Blyth, Journ. A. S. Beng. xiv. p. 589 (1845); Catal. B. Mus. A. S. Beng. p. 144. Bonap. C. G. Av. p. 282.

Orthotomus sepium, Lafres. Mag. de Zool. 1836, t. 51 (nec Horsf. v. Raffles).

Hab. Malacca.

O. cineraceus.- " Upper parts pure ash-grey, without any tinge of green ; forehead and sides of the head light ferruginons, palest on the cheeks, and there is a slight tinge of the same upon the chin; crown tinged with olive-brown; lower parts white, passing to light ashy on the sides of the breast; tail somewhat brownish, with terminal dusky band, and whitish extreme tips to its outer feathers; tibial plumes rust-coloured; the tarsi and toes red-brown; bill dusky above, pale beneath.

"Length about $4 \frac{1}{2}$ inches ; of wing, $1 \frac{8}{10}$; tail, $1 \frac{5}{8}$; bill to gape, $\frac{3}{4}$; tarse, $\frac{5}{8} . "-B l y t h$.

A specimen in the East India Company's Museum, from Malacca, has the centre of the throat and the whole of the breast and flanks lightish ash, paling to silky white on the centre of the belly; the under tail-coverts are also white; wings brown, the primaries margined exteriorly with pale dusky ferruginous, the secondaries with ashy olive; extreme edge of wing, under spurious wing-coverts and exterior margin of the latter ferruginous white, contrasting with the ash on the breast; under wing-coverts white. The wing and tarsus are both an eighth of an inch longer. Other characters as in the description above.

\section{Orthotomus longirostris, Swainson.}

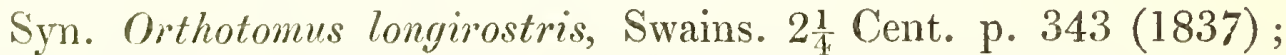
Classif. Birds, ii. p. 62. f. 135. G. R. Gray, Gen. of Birds, i. p. 162. Bonap. C. G. Av. p. 282.

Hab. S.W. Australia (Swains.). Malacca?

O. longirostris._." Cinereous; sides of the body beneath cinereous, the middle white; head, chin and thighs ferruginous; throat black; tail brownish, graduated, the latter with dusky black ends, tipped with whitish; bill and legs pale.

"Length, $4 \frac{1}{2}$ inches; wing, $2 \frac{1}{10}$; tail, beyond 1 ; base, $1 \frac{4}{10}$; bill to grape, $\frac{8}{10}$; tarsus, $\frac{9}{10} . "-$ Swains.

This species differs from $O$. cineraceus in having a black throat, and in the wing being three-tenths of an inch longer, the tarsus also leeing longer by a quarter of an inch. Mr. Swainson has given S.W Australia as the habitat of this species, which must evidently be an error. 
8. Orthotomus cucullatus, Temminck.

Syn. Orthotomus cucullatus, Temm. Pl. Col. 599. f. 3 (1836). G. R. Gray, Gen. of Birds, i. p. 162. Bonap. C. G. Av. p. 282.

Hab. Java; Sumatra.

O. cucullatus.- "Top of the head bright ferruginous; neck, jaws, and the sides of the breast of a pure ash; the neck in front, the breast, and the middle of the belly are pure white; the sides, the thighs and the abdomen of a yellow citron; back and wings of a greenish tint, the quills and tail margined with greenish ; upper mandible brown, the lower as well as the feet yellowish."-Temminck.

\section{Orthotomus longicauda, Gmelin.}

Motacilla longicauda et sutoria, Gmel. S. N. L. i. pp. 954, 997.

Orthotomus longicauda, Strickl. Ann. N. H. xiii. p. 35. Blyth, J. A. S. Beng. xiii. p. 377 ; Catal. B. Mus. A. S. Beng. p. 144. G. R. Gray, Gen. of Birds, i. p. 162. Tickell, J. A. S. Beng. xvii. pt. i. p. 298. Hutton, J. A. S. Beng. xvii. pt. ii. p. 691. Bonap. C. G. Av. p. 281. Layard, Ann. N. H. 1853, p. 262.

Sylvia longicauda et sutoria, Lath. Ind. Orn. ii. pp. 545, 551;

Gen. Hist. vii. pp. 79, 119 . Vieill. Enc. Méth. p. 456.

Malurus longicaudus, Pearson, J. A. S. Beng. x. p. 644.

Sylvia guzuratta, Lath. Ind. Orn. ii. p. 554 ; Gen. Hist. vii. p. 129. Orthotomus Bennettii et O. lingoo, Sykes, P. Z. S. (1832) p. 90. Lafres. Mag. de Zool. (1836) t. 52, 53. Jerdon, Madr. Journ. xi. p. l. Hodgs. Cat. B. Nep. p. 63.

Orthotomus ruficapilla, Hutton, J. A. S. Beng. ii. p. 504 (1833). Orthotomus sphenurus, Swains. $2 \frac{1}{4}$ Cent. p. 343 (1838).

Orthotomus sutorius, v. ruficapillus, v. sphenurus, Hodgs. Gray's Zool. Misc. (1844) p. 82.

Orthotomus sutoria et O. patia, Hodgs. P. Z. S. (1845) p. 29.

Sutoria agilis, Nicholson, P. Z. S. (1851) p. 194.

The Indian Tailor Bird.

Phutki, of the Hindoos, Jerdon.

Tuntuni, of the Bengalese, Hamilton, Blyth.

Patia, or "Leaf Bird," Nepal, Hodgson.

Hab. India generally; Ceylon; Burmese countries; Malayan peninsula?

This species is too well known to require further description.

"The Tailor Bird is tolerably common in most wooded districts, and universally spread, frequenting cultivated ground, especially gardens, groves of trees, and is also found in high jungle, in the more open spaces. It lives in pairs or in small flocks, incessantly hopping about the branches of trees and shrubs, peas and other vegetables, with a loud reiterated note, and picking various insects (chiefly ants and small larvæ) off the bark and leaves, and not unfrequently seeking them on the ground. It has the habit of frequently jerking up its tail while feeding or hopping about, and at times (especially when calling) it has the power of raising the feathers on the lower part of the throat, and displaying on either side a small black stripe. This 
has been noted by no one except Lieut. Hutton, who states, "it is only seen when the bird is in motion, and wholly disappears when in a state of rest." It has various notes, one of which sounds like twee, twee, twee, as mentioned by Col. Sykes, and another which is generally uscd when alarmed or angry, and sounds like chick, chick, chick, chicky, chick. It is a familiar bird, and ventures close to houses, but when observed becomes wary."-Jerdon.

Dr. Nicholson says, "It has a loud, short, and not unmelodious song; its general cry being 'wheet, wheet, wheet,' often repeated; but its alarm cry is like 'cheertah, cheertah, cheertah.'"

The following are a few observations on the structure of two specimens of the nest of the 'Tailor Bird, found in the garden belonging to Capt. Hearsey, by Lieut. Hutton: "The first was neatly formed of raw cotton and bits of cotton threads, woven strongly together, thickly lined with horse-hair and supported between two leaves on a twig of the Amaltás tree (Cassia fistula). These two leaves were first placed longitudinally upon each other, and stitched in that position from the points to rather more than halfway up the sides with a strong thread spun from the raw cotton by the bird, leaving the entrance to the nest at the point where they join the branch of the tree. Both of these leaves were of course green and living. Subsequently, however, they were blown down by a high wind, and being now withered, the nest appears enclosed between two dead leaves." The second specimen was at the end of a branch of the Bhela (Semecarpus anacardium), about two feet from the ground, and constructed of the same materials as the above, viz. raw cotton, cotton threads, also a little flax, and lined with horsc-hair alone; the leaves were stitched together partly with thread prepared by the bird, and partly with spun thread, and so well concealed was it, that even after Capt. Hearsey had discovered it (by accident) he could scarcely find it again to show to me. In it were found an egg and two young birds nearly fledged. These I placed, with the nest, in a trap-cage, and thus succeeded in capturing both of the old birds. The young birds are similar in colours to the adults, except that they are paler and the top of the head cinereous with a faint rufous tinge; bill yellowish. 'The eggrs are white, spotted, chicfly at the larger end, with tawny spots."

Further notices of the habits and nest of this curious bird will be found by the following authors, in the references above referred to, viz. Hodgson, Sykes, Blyth, Layard, Nicholson, \&c. 


\section{TANAGRARUMI}

\section{Outalongs Sperificus,}

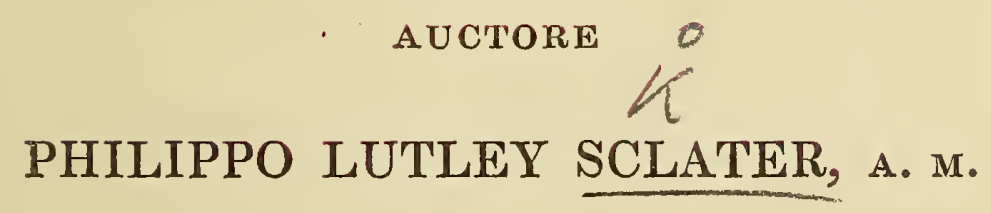

Soc. Zool. Lond. Socio.

\section{BASINGSTOKE,}





\section{TANAGRARUM}

\section{CATALOGUS SPECIFICUS.}

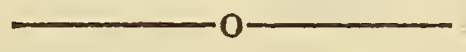

I.-Pitylus. Cuv.

1. grossus. (L.) ex Cayennâ.

2. gnatho. (Licht.) ex Brasiliâ.

II.-Periporphyrus. Reichb.

1. celæno. (Licht.) ex Mexico.

2. erythromelas. (Gm.) ex Cayennâ.

III.-Caryothraustes. Reich.

1. viridis. (Vieill.) ex Cayennâ.

2. brasiliensis. Cab. ex Brasiliâ.

3. episcopus. (Licht.) ex Mexico.

4. atroolivaceus. Lafr. ex Mexico.

IV.-Schistochlamys. Reich.

1. fasciata. (Licht.) ex Brasiliâ. 
2. capistrata. (Max.) ex Brasiliâ.

3. atra. (Gm.) ex Bras. Cayen. Bol. Trinit.

4. abeillei. (Less.) ex Brasiliâ.

V.-Chlorornis. Reich.

1. riefferii. (Boiss.) ex Columbiâ: Peruviâ.

VI.-Lamprospiza. Cab.

1. melanoleuca. (Vieill.) ex Cayennâ.

VII.-Saltator. Vieill.

1. magnus. (Gm.) ex Cayennâ: Brasiliâ.

2. similis. d'Orb \& Lafr. ex rep. Arg.

* 3. olivascens. Cab. ex Guianâ.

4. cærulescens. V. ex Bras. Mer.

5. superciliaris. (Spix.) ex Bras.

* 6. maxillosus. Cab. ex rep. Argentinâ. (?)

7. gularis. (Less.) ex Am. Mer.

8. rufiventris. d'Orb \& Lafr. ex Boliviâ.

* 9. vigorsi. Gray. ex Am. Cent. Occ.

10. icterophrys. Lafr. ex Mexico. (?)

11. orenocensis. Lafr. ex Venezuelâ.

12. aurantiorostris. V. ex Parag. Boliv.

13. atricollis. V. ex Brasiliâ. Boliv.

14. atriceps. Less. ex Mexico.

* 15. gigantodes. Cab. ex Mexico. (?)

16. magnoides. Lafi. ex Mexico.

17. albicollis. V. ex Cayennâ. 
18. striatipectus. Lafr. ex Nova Grenadâ.

19. maculipectus. Lafr. ex Novâ Grenada. 20. guadelupensis. Bp. ex Guadelupâ ins.

21. martinicensis. Bp. ex Martinico. ins.

\section{VIII.-Cissopis. Vieill.}

1. picata. (Lath.) ex Brasiliâ.

2. media. (Bp.) ex Cayennâ.

3. minor. Tschudi. ex Boliviâ.

IX.-Arremon. Vieill.

1. silens. (Gm.) ex Guianâ. Bras. Sept.

* 2. flavirostris. Sw. ex Bras.

3. polionotus. Bp. ex rep. Arg.

4. semitorquatus. Sw. ex Brasil.

5. axillaris. Sclater. ex Nov. Grenad.

6. schlegeli. Lafr. ex Am. Mer.

7. aurantiorostris. Lafr. ex Panamâ.

8. abeillei. Less. ex rep. Equat.

9. conirostris. Bp. ex Brasil.

X.-Phanicophilus. Strick.

1. palmarum. (L.) ex Hispaniolâ.

2. poliocephalus. (Bp.) ex Hispaniolâ.

XI.-Buarremon. Bp.

1. torquatus. (d'Orb \& Lafr.) ex Boliviâ.

2. assimilis. (Boiss.) ex Bogotâ. 
3. pallidinucha. (Boiss.) ex Bogotâ.

4. brunneinucha. (Lafr.) ex Bogotâ.

* 5. xanthogenys. (Cab.) ex Venezuela. (?)

6. rufinucha. (d'Orb \& Lafr.) ex Boliviâ.

7. albifrenatus. (Boiss.) ex Bogot.

* 8. albinucha. (d'Orb \& Lafr.) ex Nov. Grenad.

9. gutturalis. (Lafr.) ex Columb.

10. schistaceus. (Boiss.) ex Bogotâ.

XiI.-Pipilopsis. Bp.

1. semirufus. (Lafr. \& Boiss.) ex Bogotâ.

2. fulviceps. (Lafr. \& d'Orb.) ex Boliviâ.

\section{XiII.-Chlorospingus. Cab.}

1. opthalmicus. (Lafr.) ex Mexico.

2. flavipectus. (Lafr.) ex Bogotâ.

3. canigularis. (Lafr.) ex Bogotâ

4. olivaceus. (Bp.) ex Columbiâ.

5. flavigularis. (Sclater.) ex Bogotâ.

6. atropileus. (Lafr.) ex Bogotâ。

7. rubrirostris. (Lafr.) ex Bogotâ.

8. superciliaris. (Lafr.) ex Bogotâ.

9. verticalis. (Lafr.) ex Bogotâ.

\section{XiV.-Pyrriocoma. Cab.}

1. ruficeps. (Strickl.) ex Brasiliâ.

* 2. personata. Cab. ex Guianâ. 
XV.-Thlyporsis. Cab.

1. fulvescens. (Strick.) ex Am. Mer. (?)

2. sordida. (d’Orb \& Lafr.) ex Boliviâ.

3. ruficeps. (Lafr.) ex Venezuelâ.

XVI.-Nemosia. Vieill.

1. pileata. (Gm.) ex Cayennâ Bras.

2. guira. (L.) ex Cayennâ.

3. ruficapilla. (V.) ex Bras.

4. flavicollis. (V.) ex Cayen. Bras.

5. peruana. (Bp.) ex Peruviâ.

XVII.-Leucopygia. SW.

1. ruficollis. (Licht.) ex Brasiliâ.

XVIII.-TAChYphonus. Vieill.

1. cristatus. (Gm.) ex Guianâ: Bras.

2. delattrei. Lafr. ex Columbiâ.

3. phœniceus. Sw. ex Am. Cent.

4. rufiventer. (Spix.) ex Peruv. Bras. int.

5. coronatus. (V.) ex Bras. Mer.

6. surinamus. (L.) ex Guian: Cayenn.

7. melaleucus. (Sparm.) ex Am. Mer. Cisand.

8. luctuosus. (d'Orb \& Lafr.) ex Columbiâ.

Trinitate ins.

XIX.--Trichothraupis. Cab.

1. quadricolor. (V.) ex Bras. Mer. 
2. albicollis. (Lafr. \& d'Orb.) ex Boliv.

3. penicillata. (Spix.) ex Brasil.

XX.-Lanio. Vieill.

1. atricapillus. (Gm.) ex Cayennâ.

2. aurantius. Lafr. ex Am. Cent.

3. versicolor. Lafr \& d'Orb. ex Boliviâ.

XXI.-PhGicothraupis. Cab.

1. rubica. (V.) ex Bras.

2. rubicoides. (Lafr.) ex Mexico. (?)

3. gutturalis. Sclater. ex Nov. Grenad.

XXII.-Lamprotes.SW.

1. loricatus. (Licht.) ex Brasiliâ.

2. albocristatus, (Lafr.) ex Bogotâ.

XXIII.-ORthogonys. Strick.

1. chloricterus. (V.) ex Brasiliâ.

2. cyanicterus. (V.) ex Cayennâ.

XXIV.-Pyranga. Vieill.

1. oestiva. (Gm.) ex Am Bor. Mexico.

2. saira. (Spix.) ex Am. Mer.

* 3. livida. Sw. ex Mexico.

4. hepatica. Sw. ex Mexico.

* 5. roscogularis. Cabot. ex Am. Cent.

6. erythroccphala. Sw. ex Mexico. 
7. rubra. (L.) ex Am. Sept.

8. ludoviciana. (Wilson.) ex Am. Sep. Occ.

9. rubriceps. Gray. ex Bogotâ.

10. leucoptera. Trudeau. ex Bogotâ. Peruv.

11. bidentata. Sw. ex Mexico.

XXV.-Ramphocelus. Vieill.

1. brazilius. (L.) ex Brasiliâ.

2. dorsalis. Bp. ex Brasil.

3. nigrogularis. (Spix.) ex Rio Negro.

4. jacapa. (L.) ex Cayennâ.

5. magnirostris. Lafr. ex ins. Trinit.

6. venezuelensis. Lafr. ex Venezuel.?

* 7. affinis. (Lesson.) ex Columbiâ.

8. dimidiatus. Lafr. ex Col.

9. luciani. Lafr. ex Col.

10. uropygialis. Bp. ex Guatimalâ.

11. atrosericeus. (Lafr. \& d'Orb.) ex Boliviâ.

12. aterrimus. Lafr. ex Boliviâ.

* 13. passerinii. Bp. ex Mexico.

14. flammigerus. (Jard.) ex Col.

15 aurinotus. Lafr. ex Col.

16. icteronotus. Bp. ex Col.

17. sanguinolentus. (Lesson.) ex Am. Cent.

\section{XXVI.-Spindalis. Jard.}

1. nigricephala. (Jameson.) ex Jamaicâ. * 2. multicolor. (V.) ex Hispaniola.

3. pretrei. (Less.) ex Antillibus. 
XXVII.-Thanagra, L.

1. episcopus. (L.) ex Guianâ。

2. coelestis. Spix. ex Brasil. int. Peruv.

3. cana. Sw. ex. Bras. Sept.

* 4 glaucocolpa. Cab. ex Venez. ?

5. cyanoptera. (V.) ex Bras. Mer.

6. vicarius. Less, ex Am. Cent.

7. ornata. Sparm. ex Bras.

8. palmarum. Max. ex Cay. Bras.

\section{XXVIII.-Dubusia. Bp.}

1. tæniata. (Boiss.) ex Bogotâ.

2. cyanocephala. d'Orb. \& Lafr. ex Boliviâ.

3. olivicyanea. (Lafr.) ex Bogotâ.

4. striata. (Gm.) ex Brasiliâ.

* 5. frugilega. (Tsch.) ex Peruv. Transand.

\section{XXIX.-Compsocoma. Cab.}

1. victorini. (Lafr.) ex Nov。 Grenadâ。

2. somptuosa. (Less.) ex Peruviâ.

3. flavinucha. (d'Orb \& Lafr.) ex Boliv.

XXX.-Butmraupis. Cab.

1. montana. (d'Orb \& Lafr.) ex Boliviâ.

2. cucullata. (Jard.) ex Bogotâ.

3. chloronota. Sclater. ex rep. Equator.

4. eximia. (Boiss.) ex Nov. Grenadâ. 
XXXI.-Anisognathus. Reich.

1. igniventris. (d'Orb \& Lafr.) ex Boliv.

2. lunulatus. Du Bus. ex Nov. Grenad.

3. lacrimosus. (Dubus.) ex Col.

\section{XXXII.-Iridosornis. Less.}

J. dubusia. (Bp.) ex Bogotâ.

* 2. analis. (Tsch.) ex Peruviâ. Transand.

\section{XXXIII.-Calliste. Boie.}

1. tatao. (L.) ex Cayennâ.

2. yeni. (d'Orb \& Lafr.) ex Boliviâ.

3. colicolor. Sclater. ex Nov. Gren. (?)

4. tricolor. (Gm.) ex Brasiliâ.

5. festiva. (Shaw.) ex Brasil.

6. fastuosa. (Lesson.) ex Brasil.

7. cyanoventris. (V.) ex Brasil.

8. aurulenta. (Lafr.) ex Bogota.

9. arthusi. (Less.) ex Venezuelâ.

10. icterocephala. (Bp.) ex rep. Equator.

11. thoracica. (Temm.) ex Brasil.

12. schranki. (Spix.) ex Peruvia. Bras. int.

13. guttulata. (Bp.) ex Columbiâ.

14. punctata. (L.) ex Cayennâ.

15. rufigula. (Bp.) ex rep. Equator.

16. xanthogastra. Sclater. ex Nov. Grenadâ.

17. graminea. (Spix.) ex Cay. Bras.

18. flava. (Gm.) ex Brasiliâ.

19. ruficapilla. Sclater. ex Bogota. 
20. cayana. (L.) ex Cayenne.

21. cyanolæma. Bp. ex Rio Negro.

22. cucullata. (Sw.) ex St. Thomas.

23. castanonota. Sclater. ex Bras. Mer.

24. peruviana. (Desm.) ex Bras. Or.

25. cyanoptera. ( $\mathrm{Sw}_{\circ}$ ) ex Venezuela.

26. larvata. Dubus. ex Am. Cent.

27. Iunigera. Sclater. ex Rio Negro.

28. lamprotis. Sclater. ex Boliviâ.

29. parzudakii. (Lafr.) ex Bogota.

30. chrysotis. Du Bus. ex Peruv.

31. gyrola. (L.) ex Cayennâ.

32. gyroloides. (Lafr.) ex Peruv.

33. desmaresti. Gray. ex Venez. Trini.

34. brasiliensis. (L.) ex Brasil.

35. flaviventris. (V.) ex Cayennâ. Trini.

36. boliviana. (Bp.) ex Boliviâ.

* 37. nigrocincta. (Bp.) ex Peruv. (?)

\section{XXXIV.-Procnopis. Cab.}

1. atrocærulea. Tsch. ex Peruviâ.

2. ruficervix. (Prevost.) ex Bogotâ.

3. atricapilla. (Lafr.) ex Nov. Grenadâ.

4. argentea. Tsch. ex Peruviâ.

5. nigroviridis. (Lafr.) ex Bogota.

6. thalassina. (Strickl.) ex Nov. Gren.。

7. cyanicollis. (d'Orb \& Lafr.) ex Peruv.

8. labradorides. (Boiss.) ex Bogotâ.

9. xanthocephala. (T'sch.) ex Peruv. 
XXXV.-Diva. Sclater.

1. vassori. (Boiss.) ex Nov Grenad.

2. albiventris. Sclater. ex Nov. Grenad.

XXXVI.-Pipreidea. Sw.

1. melanonota. (V.) ex Brasiliâ.

2. castaneoventris. Sclater. ex Boliviâ.

XXXVII.-ChLOROChrysa. Bp.

1. phænicotis. Bp. ex rep. Equator.

2. calliparæa. (Tsch.) ex Peruv.

XXXVIII.-Tanagrella. Sw.

1. velia. (L.) ex Cayennâ.

2. elegantissima. Verr. ex Nov. Grenadâ,

3. cyanomelas. (Max.) ex Brasil. Or.

4. calophrys. (Cab.) ex Brasil. int.

XXXIX.-Chlorophonia. Bp.

1. viridis. (V.) ex Bras.

2. frontalis. Bp. ex Equator.

3. occipitalis. (Du Bus.) ex Mexico.

4. pretrei. (Lafr.) ex Bogotâ.

XL.-Euphonta. Vieill.

1. musica. (Gm.) ex Hispaniolâ.

2. flavifrons. (Sparm.) ex Antill.

3. nigricollis. (V.) ex Am. Mer. Cisand. 
4. elegantissima. ex Mexico.

5. chlorotica, (L.) ex Cayennâ.

6. trinitatis. Strickl. ex Trinit. ins.

7. minuta. Cab. ex Cayennâ.

8. chalybea. (Mikan.) ex Bras.

9. xanthogastra. (Sund.) ex Bras.

10. ruficeps. d'Orb \& Lafr. ex Bol.

11. concinna. Sclater. ex Nov. Grenad.

12. violacea. (L.) ex Bras.

13. hirundinacea. Bp. ex Am. Cent.

14. laniirostris. d'Orb \& Lafr. ex Boliv.

15. melanura. Sclater. ex Rio Negro.

16. rufiventris. (V.) ex Brasil.

17. pectoralis. (Lath.) ex Brasil.

18. cayana. (L.) ex Cayennâ.

19. jamaicæ. (L.) ex Jamaicâ.

XLI.-Stephanophorus. Strick.

1. leucocephalus. (V.) ex Parag. Bras. Mer. 


\section{ANNOTATIONES.}

Species ab asterisco notatas nondum vidi.

(1) Schistochlamys abeillei est Pyrrhula abeillei Leeson Rev. Zool. 1839, p. 40. Diucopis leucophæa Bp. Consp. p. 491. Orchesticus occipitalis Cab. Mus. Hein. p. 143.

( $\left.1^{*}\right)$ Arremon axillaris mihi sp. nova est $\mathrm{A}$. semitorquato maximè affinis, sed axillis lœtissimè aureis.

(2) Chlorospingus olivaceus est Poospiza olivacea Bp. Mus. Paris. Consp. Av. p. 474.- -species prœeedentibus affinissima.

(3) Thlypopsis ruficeps est T. fulviceps Cab. Mus. Hein. p. 138. Tachyphonus ruficeps Lafr. Rev. Zool. 1848. p. 173.

(4) Tachyphonus surinamus est Tanagra surinamus Linn. S. N. 1. p. 297. Tachyphonus martialis Temm. T. desmaresti Swains. T. ochropygos Cab. T. surinamensis Lafr. ex Brissonio, cum Tachyphono cristato minimè confundendus.

(5) Orthogonys cyanicterus est Pyranga cyanictera Vieill. Cyanicterus venustus $\mathrm{Bp}$. sed me judice ab $\mathrm{O}$. chlorictero genere handquaquam disjungendus.

(6) Pyranga saira. est Tanagra saira Spixi Ar. Bras t. 48. fig. 1. Pyranga azarœ auctorum recentiorum.

(7) Pyranga dorsalis Bp. ex Brasiliâ sp. nova. Ramph. brazilio affinissima : sed dorso medio obscuriús coccineo.

(8) Buthraupis chloronota mihi sp. nov. ex rep. Equator. similis est B. cucullatæ sed multo major, dorso toto viridi unicolore, neque uropygio coeruleo.

(9) Calliste graminea est Tanagra graminea Spix. Av. Bras t. 53. fig. 2. Ixothraupis pusilla Bp. Calliste virescens mihi. 
(10) Procnopis ruficervix. Ab hac specie Calliste leucotis mihi Cont. Orn. 1851. p.58. non potest separari.

(11) Diva mihi genus novum Callistæ et Euphoniæ intermedium. Typus sit Tanagra (Euphone?) vassorii Lafr. et Boiss. Rev. Zool. 1840. p. 4. Aglaia diva Less. Echo d. M. S. 1844. p. 57. Procnopis vassori Bp. Species secunda hujus generis erit Piproidea albiventris mihi Rev. Zool. 1852. p. 8. et Cont. Orn. 1852.pl. 100.

(12) Euphonia flavifrons. est Euphone organiste Desm. Tan. t. 19 20. Emberiza flavifrons Sparm. Mus. Carls. no. 92. Tanagra flavifrons Lath Euphonia musica mihi Cont. Orn. 1852. (pars) Cyanophonia sclateri Bp. in Mus. Parisiensi.

Mas. violaceo-ater : dorso imo et corpore subtùs aurantio-flavis : pileo cyaneo: fronte loeté flavo, nigro marginato.

Fæm. olivacea : dorso postico et abdomine flavescentiore: fronte flavo. ex ins. Porto Rico dictâ. Mus. Paris.

(13) E, concinna mihi sp. nov. E. chlorotiæ affinis; sed minor; intensè violacea; rectricibus non albo maculatis; ex Nov. Grenad.

(14) Euphonia hirundinacea Bp. est Euphonia laniirostris mihi Cont. Orn. 1851. p. 86 nec Lafr. et d'Orb. teste illius avis exemplo in Museo Derbiano conservato.

(15) Euphonia Jamaicæ est Euphonia cinerea Lafr. Pyrrhuphonia cinerea Bp.

P. L. S.

Londini, 1 Kal. Jan. A. D. 1854.

R. Cotlle, Printer, Basingstoke. 


\section{MÉMOIRE}

SUR

\section{LES OISEAUX GRAND-VOILIERS}

DE I I

SOUS-F IMILLE DES LARIENS 
- 
No 11. -2854.

\section{ZOO LO G I E.}

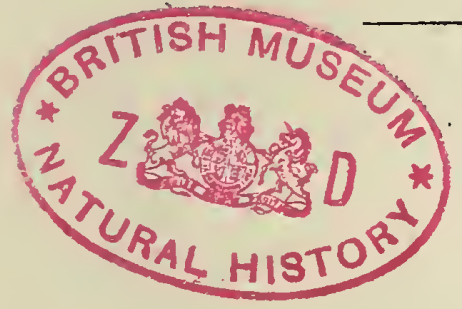

\section{NOTES \\ S UR}

\section{LES L ARID ES}

Par S. A. le Prince C.-L. Bonaparte.

M. le notaire Bruclı vient enfin de passer l'acte qu'on attendait depuis longremps et dont nous avions revu ensemble les minutes, avec ses clients de vieille date, les Lamiens. Ce Mémoire, en lui restituant son véritable nom, publiè dans le second numéro du Journal d' $\mathrm{Or}^{-}$ nithologie de M. Cabanis, est tel qu'on l'attendait de la capacité de ce zélé directeur du Musée de Mayence. L'importance que l'on doit attacher à l'éminent travail qui a été l'occupation principale, pendant nombre d'années, de son auteur, me détermine à relever les quelques erreurs et omissions que j'ai pu y reconnaitre.

Il serait heureux que M. Bruch, malgré l'état fâcheux d'infirmité dans lequel il se trouve, se décidàt à mettre éralement en orddre les Stenners, qui laissent encore 
plus à désirer que les laniens. L'amour de la science. qui fait surmonter toutes les déceptions, tous les mécomples, et mêne les douleurs les plus poignantes, les douleurs morales !... doit à plus forte raison faire oublier les douleurs physiques. La famille entière des Landes serait ainsi passée en pevue, au moyen de cel arrangement des Strensess que la science réclame de 11. Bruch. Il a déjà, en effet, traité des quelques espèces formant, à notre avis, la sous-famille des LesThagers, qui n'est, pour ainsi dire, qu'un appendice des Lamers. Celle des Runchoriens est dans des condilions semblables, par rapport aux Sternexs, sauf qu'elle suit au lieu de précéder.

Il est facile de se convaincre lous les jours davantage que c'est par séries parallèles qu'il convient le mieux de disposer les êtres organisés, afin de suivre et de représenter le pius lidèlement possible les lois de la nature. La première des séries que nons venons d'indiguer parmi les Larides, celle des Laraens, se subdivise elle-mème en drax : Larete et Xemere.

\section{Sublamilia AVIUM 22\%. I arune.}

Series A. Laree.

Series B. Nemear.

1. Procellarus, Bp. Oc. 1. 12. Adelarms, Bp. a. Africaniz.

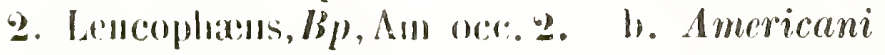

5. Blasipus, Bpp. As Am. 2. 15. Arricilla, Bpp. Amer. ¿.

4. Gabianus, Bp. Austr. 2. 1\%. Ichthy:nens, Kaup. Medit. 1.

¿. Larns, L. Cosm. 9. 18̈. Gavia, Briss. nee Bruch.

(i. Laroides, Brehm. Coim. 10. a. Melagavia, Bp. Cosm. 9.

7. Gavina, Bp. Cosm. 7. b. Gáravia, Bp. Cosm. B.

ฉ. Ciclastes, Bp. Cosm. 7. c. Cirrhocephalus, Bp. Am. 2.

9. Pigophila, Lanp. Arct. ‥ 16. Chroicocephat us, Eyton. 1.

10. Rissa, Brumn Arel. 3. 17. Creagrus, Bp. Am. s. oce. 1.

11. Rhodontethia Heregil. Are.1. 18. Xema, Leach. M. aret. 1. 
La principale addition que nous ayons à faire au Mémoirc de M. Bruch est certes notre Procellarus neglectus, singulier genre dont on ne connaît encore que lejeune, obscurément conservé depuis 1831 dans le Musée de Paris, époque à laquelle il fut apporté par M. d'Orbigny de je ne sais quelle localité des mers du Sud. Ce Larien montre une forte tendance vers les Lestrigiens, sans pouvoir être pris pour l'un d'eux. Si. contre toutes les règles de la nomenclature, nous donnons un double nom à ce singulier genre, c'est pour moins mécontenter lss puristes, qui pourront substituer le second, Epitelarus, à celui que nous préférons, et éviter peut-être à $M$. Cabanis d'en donner un de sa façon à un oiseau trop longtemps nég̣!igé dans nos galeries nationales. Voici ses caractères génériques et spécifiques:

Rostrum brevissimum, compressum : digitorum membrana valde emarginata : alce caudam brevem vix excedentes.

Statura L. cani : fuscus, capite subrufescente; subtus albido-fuscescens; remigum apicibus speculum constituentibus, uropygio, tectricibusque candalibus albis : cauda alba, fascia lata subapicalifusca: rostro flavo, upice fusco. An adultus?

Passons à la revue du llémoire de $\mathrm{M}$. Bruch, et occupons-nous d'abord des genres. Nous qui croyons devoir conserver le nom linnéen Larus à un des groupes les plus restreints, nous nommons ainsi le genre Dominicanus de Bruch, et adaptons le nom $\mathbf{L} \boldsymbol{C}$ roides de Brehm an Glaucus de Bruch. Outre que ce nom générique est préoccupé parmi les Animaux invertébrés, le nom Plautus, emprunté à Klein par Reichenbach, aurait lui-même la priorité sur celui de notre auteur. Nous le concevons d'ailleurs dans des limités moins étendues que lui, et nous nommons Gavina, Bp. les dernières espèces, que nous en détachons. 
Le quatrième genre de Bruch est appelé par nous depuis Iongtemps Gelastes; ce Gavia de Bruch n'est d'ailleurs ni celui de Boie, qui est le genre Pagophila. Kaup., (Cetosparactes, Macuill.), ni celui de Swainson. qui appartient aux Noddis, ni celui de Brisson, auquel ce nom doit être conservé.

Le sixième genre de M. Bruch est coupé par moi en deux : chacune de ces espèces formant le type d'un genre séparé, Xema restant à Sabini, Leach, pour la. quelle il fut créé, et furcatus, Néboux (non Lesson) ćtant celui de Creayrus, Bp.

Le septième genre, auguel M. Bruch élend le nom de Chroicocephalus, Eyton, comprend mes genres:

$1^{\circ}$ Ichthyaetıs, Kaup.. pour la seule espèce ainsi nommée par l'allas:

$2^{\circ}$ Atricilla, Bp., pour trois espèces, dont une non admise par 11. Bruch, et l'autre confondue à tort par lui avec le $L$. serramus, de Tschudi, qui est son personatus, Natterer ;

$5^{\circ}$ Gavia, Brisson. pour la grande masse des espèces ayant pour type le ridibundus, L.;

$4^{\circ}$ Et finalement Chroicocephulus pour la petite espèce à bec mince, L. minutus, qu'il vaut mieux laisser seule sans même lui adjoindre ses plus proches alliés, le $\boldsymbol{L}$. bonapartii et le subulirostris.

M. Bruch n'a pas tout à fait bien compris ses trois derniers genres 10, 11 et 12, Adelarus, Blasipus et Leucophous, qu'il a pris de moi, ayant réparti les espèces autrement que je n'en avais l'intention, et que je n'en comprends les alfinités.

Mais hâtons-nous d'arriver à la partie la plus essentielle et la mieux trailée par notre auteur, à la critique des especes. Dans son premier genre Gabiamus, Bp., II. Bruch n'en arlmet qu'une, qu'il nomme pacificus. Lath :en lui donnant pour synonymes leucomelas, Vieill. 
et georgii, Vig., auxquels on peut ajouter, comme quatrième appellation, bathyrhynchus, Macgillivray. Ces synonymes cependant doivent être, suivant moi, répartis entre deux espèces qui se distinguent fort bien par la taille.

Les espèces du second genre, mon Larus propre, sont bien nommées et déterminées par notre auteur. Je n'ai autre chose à faire remarquer, si non que, voulant conserver le nom spécifique de dominicanus à une de ces espèces (ce que ne pouvait faire M. Bruch, qui l'emploie pour le genre, et le cite d'ailleurs sous trois espèces différentes), je l'applique exclusivement au D. vociferus, Bruch, parce que je crois que c'est à cette race du Brésil que l'a donné Lichtenstein pour la première fois.

J'ai aussi à ajouter une espèce nouvelle gu'on pourrait désigner comme le Larus fuscus du Chili, et que je nommerai Larus verreauxi, Bp., ex Chili. Minor L. Fusco cui similis : alis longissimis, remigibus nigris subunicolo. ribus, macula singula alba subapicali : rostro valde robustiore, fluvissimo.

On sait que le seul Montague a donné au fuscus, L. (flavipes, Neyer), le nom d'argentatus, généralement appliqué à des espèces plus grandes de Larus et de Laroüdes.

Le troisième genre de Bruch. qui finit pour moi après sa vingtième espèce, est très-bien mis en ordre par notre auteur. Il nomme consul, d'après Boie, sa première espèce, ne pouvant se servir, comme nous, du nom spécifique glaucus, qu'il emploie pour le genre. II ne faut pas confondre le leucopterus, Faber (qui est le glaucoides, Temm.), avec celui de Vieillot; comme aussi l'argentatoides, Richardson., (nonne potius Bonaparte?) propre à.l'Amérique du Nord, avec les prétendus argentatoides d'Europe, qui encombrent les collections. Les nuances assez difficiles à saisir entre les espèces dont 
nous venons de parler, et surtout entre elles et le glaucopterus, Kittlitz, le glancescens, Licht. et le glacialis, Benicken, sont admirablement établies par notre auteur. Par contre, je ne pense pas que sa dix-septième espèce, michahellesii, Bruch, diffère de sa huitième, len. cophoeus, Licht.

C'est des quatre dernières espèces du troisième genre de M. Bruch que je forme mon genre Gavina. Ajoutons-y deux espèces nouvelles: $1^{\circ}$ Gavina kamtschatschensis, Bp.. qui est la race kamischadale du Larus camus, L. . comme le lacrymosus, Licht. en est la race africaine, et le zonorhynchus, Richardson, la race nord-américaine. $2^{\circ}$ Gavina bruchi, Bp., race mexicaine, à bec romarquablement court de ce même $\boldsymbol{L}$. camus. Je ne connais pas le Larus heini, Homeyer, de Grèce, qui aurait le bec du camus, plus long même et plus grêle, avec les pieds du prétendu L. michahellesi.

Le quatrième genre de M. Bruch, pour lequel je ne conçois pas qu'il ail préféré le nom de Gavia à celui que j’ai proposé, Gelastes, a pour type cette belle espèce de la Méditerranée, pour laquelle il adopte le nom spécifique de Gelastes, Licht., passant sous silence ses nombreux synonymes. Le seul qui puisse dispuler la priorité au nom de lambruschinii, sous lequel je l’ai figuré dans ma Faune italienne, après avoir adoplé comme générique celui de Gelastes, est le nom encore douteux de rubriventris, Vieillot, que je fais ici revivre pour la première fois. Ajoutez encore aux synonymes celui de L. melemolis, Reichenb., qu'il ne faut pas confondre avec L. migjotis de Lesson, qui est un jeune Chroicocephalus minulus.

Je suis prêt à acceprer comme Gelastes hartlaubi la nouvelle espèce du cap de Bonne-Espérance, tout en doutant qu'elle s'étende jusqu'à la côte indienne; mais je l'adopte précisément parce que je ne crois pas qu'elle 
ait rien à démèler avec Larus peocephalus, Sw., qui est un Chroicocephalus de Bruch, rcprésentant sur les côtes d'Afrique notre ridibundus d'Europe.

Ajoutez ici une nouvelle espèce du Muséum de Paris, que j'ai nommée Gelastes corallinus à cause de son bec encore plus éclatant que les plus beaux de ses congénères. Elle nous mène directement au jamesoni du professeur Wilson, d'Edimbourg, qu'il ne faut pas confondre avec le célèbre ornithologiste américain, lui aussi Ecossais, ni avec les membres d'une autre famille. Wilson qui patronnent si largement dans cé moment la scicnce à Philadelphie. Qui ne connaît le Larus jamesoni de la Nouvelle-Hollande, qui est celui de Wilson, de moi et de tutti quanti? C'est bien légèrement, et d'après des doutes émis verbalement sur des espèces voisines, que M. Bruch m'accuse de vouloir changer le nom de ce Laride, et de transporter le sien à son andersoni. C'est évidemment à ce dernier plutòt qu'à son jomaré que se rapporte une espèce que j’avais considérée comme nouvelle, et à laquelle j’avais voulu appliquer le nom d'un ami commun, aussi savant que laborieux et modeste, et dont il ne peut plus être quesiion dans cette occasion.

Quant à mon Gelastes gouldi, d'ailleurs inédit, au lieu d'être plus petit que le jamesoni, comme le suppose gratuitement M. Bruch, il est plus grand, et se trouvé sur les côtes septentrionales de la Nouvelle-Hollande, tandis que le jamesoni paraît confiné aux côtes méridionales de ce continent. Les excellentes espèces de M. Bruch, andersoni et pomaré, terminent convenablement le beau genre Gelastes. Je n'ai rien à observer sur le douzième genre, Rissa, Brunnich, de M. Bruch, sinon que les espèces méritent encore d'être comparées, ne fussent que pour mieux en établir la synonymic. Larus niveus, Pallas, par exemple, ne. serait-it pas le 
mème que brachyrhynchus de Gould? et à cause de ce nom même ne vaudrait-il pas mieux appeler kotzebui. comme je l'ai fait dans mes manuscrits, la bonne espèce des côtes nord-ouest d'Amérique, à laquelle on applique le nom plus que douteux de brevirostris, Brandt. J'ai vérifié, de manière à n'en pouvoir douter, que le fameux Pulo-condor de Sparrmann, qui a tant intrigué les ornithologistes, n’était qu'un jeune Rissa: mais est-il bien vrai qu'il provienne des climats chauds de l'Asie?

On a vu plus haut ce que je pense des deux espèces dı genre Xema; du Chroicocephalus, Eyton, si amplifié par M. Bruch, ef que je restreins, au contraire, au seu! L. mimutus de Pallas (pygmous, Bory,-nigrotis, Less.); de l'Ichthyaetus, et des trois espèces de mon Atricilla: de sorte que mes observations ne porteront que sur les Xémés, que je conserve encore dans mon genre Gavia, Bp. (ex Brisson), si différent de celui de M. Bruch. Parmi elles, se distingue encore un groupe à têtes noires dont le L. melanocephalus, Natterer, est le représentanten Europe, et le vrai serranus, Tschudi (personatus, Natterer) de l'A mérique méridionale, la plus grande espèce. A ce groupe appartiennent te L. cucullatus, Licht., des plus chaudes parties du Nouveau-Monde, auruel je ne pense pas que M. Bruch ait raison de réunir le $L$. pipixan, Wagl., du Mexique. Il faut y ajcuter encore le franklini. Richardson, de l'Amérique plus boréale, le kitulitzi, Bruch, du Chili, que je ne connais pas, et le L. melanorhynchus, Temm., que M. Bruch ne veut pas admettre, el qui peut-être ne diflère pas d'une des espèces déjà énumérées. Cionsultez la planche coloriée de Temminck, sans oublier que dans les collections se trouvent sonvent, sous ce nom, des Larus bonapartii à bec noir, des L. framklini, el d'antres espèces, et qu'on le lait tantôt venir de l'Amérique du Nord, tantôt du Chili. 
Pcurquoi ne serait-ce pas le $\boldsymbol{L}$. kittlitzi, dont l'original est conservé dans le Muséum de Pétersbourg?

Le second groupe, celui des véritables Gavia, a pour type et représentant en Europe le Larus ridibundus, L., dont il est irnpossible d'éloigner sa petite race, Larus capistratus, Temm., aboli par ce patriarche de l'Ornithologic, qui vient, sans le vouloir, de le faire revivre dans son tenuirostris; car cet oiseau n'est pas, comme on le croit généralement, mon Gelastes lambruschinii, mais bien la Gavia capistrata en plumage d'hiver. C'est encore, en cet état, le $L$. nigrotis de Reichenbach; et bien loin d'être un oiseau septentrional, comme l'avait proclamé Temminck, qui naturellement n'a pu retrouver dans le nord que de véritables ridibundus, c'est une espèce essentiellement méridionale. Une belle série dans tous les âges se trouve au Musée de Francfort.

Notre ridibundus d'Europe est représenté aux GrandesIndes par le brunnicephalus de Jardine, que les puristes appelleront brunneiceps, et sur les côtes occidentales d'Afrique par le petit $L$. pcocephalus, Sw., que je concẹvrais que M. Bruch eût réuni à son proche congénère capistratus, plutôt qu'au Gelastes hartlaubi du Cap, si différent.

L'Amérique nous offre aussi deux espèces de ce groüpe, le glaucotes, Meyer (albipennis, Licht.), du Chili, si semblable au ridibundus et si absurdement nommé cucullatus dans la plupart des Musées de l'Angleterre et de l'Irlande, et le maculipennis, Licht., du Brésil. Nous appelons encore l'attention des naturalistes voyageurs sur l'Oiseau ainsi désigné, quoiqu'il nous soit à peu près prouvé que c'est le plumage d'hiver de l'élégant Larus cirrhocephalus, Vieillot, à capuchon d'un gris tellement clair qu'il se distingue à peine du blanc de neige du reste du plumage. Deux races se font distinguer par la taille dans ce longipenne dụ Brésil et du Paraguay a 
fint-il les considérer comme distinctes el applifuec plus particulièrement à la grande le nom de cirrhocephalus, Vieillot, réservant pour la petite le synonyme poliscephalus, Wied, sous lequel l'a figuré Temminck, dans ses planches coloriées? Ces oiseaux, à la rigueur, peuvent être considérés comme types d'un sous-genre a part. A propos du mauvais nom poliocephalus, disons, qu'outre la confusion créée par l'espèce douteuse de Swainson, je ne crois pas que M. Bruch ait raison de citer un poliveephalus, Temm. comme synonyme de l'atricilla, I. On aura voulu traduire en grec le $110 \mathrm{~m}$ phumbiceps, de Michahelles, qu'une erreur typographique a changé en plumiceps.

Rien aux espèces de Pagophila el de Rhodostethiu.

Quant au disième genre, Adelarus, adopté par M. Bruch, de moi, les espèces a fricaines sont les typiques. II est diflicile d'en éloigner fuliginosis, Gould, et betcheri, Vig., confondus à tort par M. Bruch. Un superbe exemplaire du premier se voit dans le llusée de Franclort; mais, quant aux autres espèces, leurs affinités et analogies sont beacoup plus complexes et difficiles a établir. Le Larus heermami, Cassin, de la Californie. malgré son apparente ressemblance avec le bridgesi, doil phuiòt se ranger sous Lencophens avec homutorliynchus; el le L. melanurus, ou crussirostris, quirs'éloigne tant du bridgesi par ses cuuleurs et par son bee. doit, au contraire, lui être réuni, etant, par une heureuse coincidence, un véritable İlasimus à gros bee.

Que bridgesii soit douc, comme M. Bruch l'a voulu, le type de mon genre Bblasimes; qu'hematorhynchus le soit, par la menteraison, de mon genre Lencophecus, puisqu'il a ché le premicr à les publier el à les caractériser. Mais proctamons hautement que ees deux genres doivent chacun s'ouriehir d'une espèce qui, au premier coup d'oxil, es lom de ressembler a soul lype congénère; 
que, malgré des différences plus apparentes que substanlielles, malgré la couleur générale. claire dans homatorhynchus, foncée dans heermanni; malgré lo bec rouge. robuste et anguleux chez le premier, noir, grêle et presque linćaire dans le dernier. ces deux espèces doivent ètre réunies génériquement. Dans ce cas.. melunurus et bridgesii se trouvent réunis ensemble par les mêmes raisons, tout en différant par. les mêmes caractères superficiels; de sorte que l'on peut mathématiquement étahlir cette proportion : Blasipus melanurus est à Leucopheus homatorhynchus ce que Blasimus bridgesii est à Leucophusus heermanni.

Ajoutez aux synonymes de Blasimus bridgesii, espèce qui se trouve an Pérou et aux ìles Gallapagos, le synonyme antérieur, mais non publie, de polios, Natterer; et ajoutez au Némoire de M. Bruch ce que lous les ornithologistes savent, que L. hamatorhynchus, King, est aussi L. scores! ii, Traill. 


\section{-}




\title{
SYSTEMATISCHE ÜBERSICHT
}

\author{
DER

\section{VÖGEL NORD - OST - AFRIKA'S}

MIT EINSCHLUSS DER ARABISCHEN KÜSTE

DES

\section{ROTHEN MEERES UND DER NIL-QUELLEN-LÄNDER}

SÜDWÄRTS BIS ZUM IV. GRADE NÖRDL. BREITE.

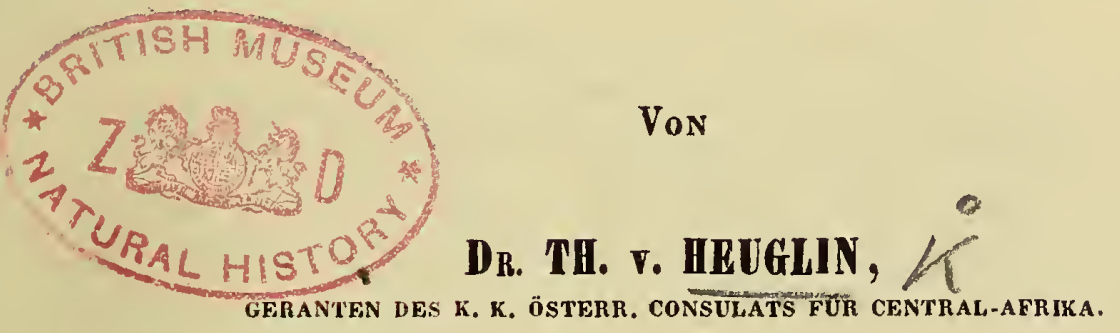

(Aus dem Februarhefte des Jahrganges 1856 der Sitzungsberichte der inathem.-naturw. Classe der kais. Akademie der Wissensehaften [Bd. XIX, S. 25:5] besonders abgedruckt.) 


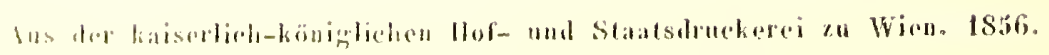


Systematische Übersicht der Vögel Nord-Ost-Afrika's, mit Einschluss der arabischen Küste des rothen Meeres und der Nil-Quellen-Lünder südwärts bis zum 4. Grade nördl. Breite.

\author{
Von Dr. Th. v. Hi e u gli in, \\ Géranten des k. k. österr. Consulats für Central-Afrika.
}

(Vorgelegt in der Sitzung vom 19. Juli 1855.)

A n m. Die mit * bezeichneten Arten sind in R üp pell's systematischer Übersicht der Vögel N. 0. Afrika's nicht aufgenommen.

\title{
1. ORDNUNG. RAPACES (RAUBVÖGEL).
}

\section{A. VULTURIDAE.}

1. Gypaëtos (R a y) meridionalis, Key s. et Blas.

R ü p p e ll, Syst. Übers. t. 1. - He u g li n, Beitr. t. 1. Findet sich nicht selten paarweise und in grösseren Gesellschaften in den höheren Gebirgen von Arabien (Gebel-Serbal und Sinaï) und Abyssinien, und kommt ohne Zweifel auch in den nubischen Bergen und längs der Küste zwischen Suez und Sauakin vor. In Abyssinien fand ich ihn einzeln bei Gondar und auf den Hochebenen von Woggara, auf den Gebirgen von Simehn aber in so grosser Anzahl, dass ich in 7 Tagen acht Stücke erhielt und vielleicht ebenso viele andere angeschossene, die nicht verfolgt werden konnten, verlor. Er lebt hauptsächlich von Überresten von Schlachtvieh, nimmt aber auch im Nothfalle mit Aas vorlieb. - Dass der Bartgeier Ziegen und Schafe angreife — wie R ü p p ell sagt, — kann ich nicht bestätigen, blos ein einziger von mir untersuchter hatte Stachelratten gefressen, in dem Magen aller Übrigen fand ich Haut- und Knochenreste von Schlachtvieh.

Auch ist die Iris nicht "s chön fe uer o th" (R ü p p. Syst. Übers. S.3), sondern s chmutzig-blas s elb mit sigellackrothem Ring am Rande. Heisst auf amharisch "Amora“, arab. petr. Büdj $\left(\varepsilon^{\wedge}\right)$.

2. Neophron (Savigny) Percnopterus, Linn.

B uff. Pl. enl. t. 407 u. 429. - N a u mann, Vög. Deutschl. t. 3. Su sem ihl, Vög. Europ. t. 4. - He u g lin, Beitr. t. 1 das Ei. — Ist sehr 
häufig in ganz Nord-Ost-Afrika, aber nicht oder wenigstens sehr seltrn auf Jem weissen Flusse. - Brütete während der Regenzeit - im Juli - 1832 in der Oasis $\mathbb{E}$ l-Gab, westlich von Dongola auf Sunt-Bäumen und im Februar 183\% erhielt ich seine Eier auch bei Edfu in Ober-Ägypten an-

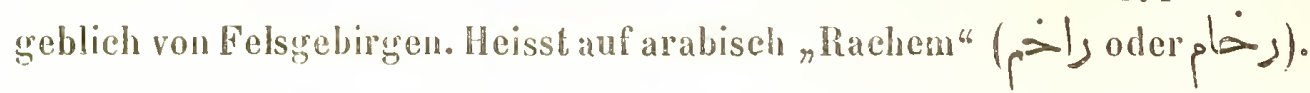

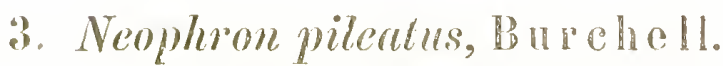

Catharles Ironaches, Te mm. Pl. color. t. 222. - Selten nördlich vom $15^{0}$ 1. B. Gemein in Kordofán, Semaar und Abyssinien, oft in Gesellschaft mit dem Vorhergehenden. Die machte Kopfhaut ist bei alten Vügeh glänzend violett. Ein gelblichweisses Gefieder, ähnlich dem des alten $N$. Percopterus, ist mir nie bei dieser Art vorgekommen.

4. Gyps ( $\mathrm{S}$ a vign $\mathrm{y})$ fulva, Linn.

Naum. V.D.t.2. - N a um. Nachtl. t.338. - Le Vaill, t. 10? - Gemein in Ägyten, Nubien und Abyssinien. Seine Heimath sind hauptsïchlich kahte Felsgebirge, von wo aus er sich aber weit in den Ebenen verfliegt. In Simehn trafich ihn über 11000 Fuss hoch noch an. In Kordofín und am weissen Flusse scheint er durch die folgende Art vertreten zu sein.

An m. Alle grösseren Geier heissen auf arabisch, "Nissr" $($,

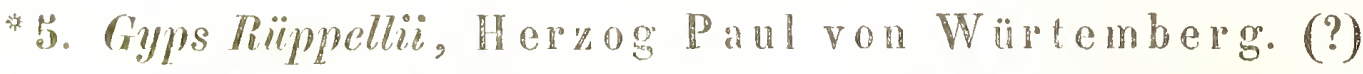

liüp. All. t. 22 als $\mathbb{V}$. Kolbii. - In ebenen Gegenden und vorriiglich in Waildern und mit Hochbäumen besetzten Steppen südlich vom 16 - $17^{\circ}$ n. B. - I I bin leider jetzt nicht im Stande die Unterscheidungs-

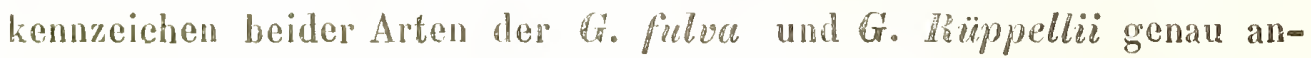
greben zu können. Die letrlere ist constint grösser, hat immer einen horngelben Schnabel im Alter und ist nie so fahl rostgelb wie Go fulva, sondern dunkler und die schuppenförmige zeichnung auf den Federrindern ist immer vorherrschend und mehr oder weniger deutlich ausgesprochen.

(1) der eigentliche Vullur hollie in Nord-Ost-Afrika rorkommt, kann ich nicht angehen. Ieh hesitze übrigens einen sehi grossen Geier aus Kordofín, der mil ersterer Art übereinzustimmen seheint.

(i. Ciyps hengalensis, Lath.

V. lenconolos, J. diray, Thd. "zoviog. L. 14.-V. moschalus, Herz. Panul v. Würtemberg. - Vinzeln in Kordofín, ann blanen und weissen Fluss, häuligg aher in Ost-Sennaar und West-Abyssinien.

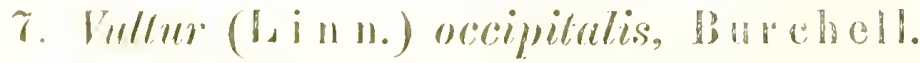

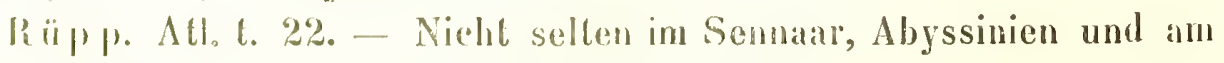
liahe ol itharl, aber nie in grossen Gesellseharten.

8. lullur cinerens. Hinn.

Na um. V. Bentsch. 1. 1. - Hescr. de l'legple Ois. t. 11. - Sehr

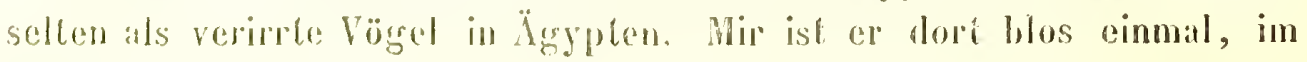


October 1851, bei Beni-Suéf auf dem freien Felde sitzend, vorgekommen.

9. Otogyps (G. R. Gra y) auricularis, D a ud.

$V$. aegipius $\mathrm{S}$ a vign y. - V. nubicus G riff. - Le V a ill. Ois. d'Afr. t. 9. - In Ober-Ägypten und Nubien sehr gemein, seltener in Kordofán und Sennaar, im Innern von Abyssinien nicht von mir beobachtet.

Die Hautfalte am $\mathrm{Ohr}$ ist bei den meisten Exemplaren ganz unscheinbar, zeigt sich aber bei längerer Gefangenschaft mehr und mehr. Doch sind mir auch freie Vögel vorgekommen, bei welchen diese sehr deutlich ausgesprochen und über $2^{\prime \prime}$ lang war.

\section{B. FALCONIDAE.}

10. Buteo (Briss.) vulgaris, Bechst.

Buff. Pl. enl. t. 419. - Na um. V. D. t. 32 und 33. - Von mir blos einzeln im Winter in Ägypten beobachtet, nach Dr. R ü p pell, „überall in N. 0. Afrika."

* 11. Buteo minor, Heugl.

F. Tachardus. Shaw (?) - Gleicht dem F. Buteo in Färbung vollkommen; ist aber schlanker und wenigstens um $1 / 4$ kleiner; das grösste Weibchen meiner Sammlung ist $\mathbf{1}^{\prime} \mathbf{5}^{\prime \prime}$ lang. - In Nubien, Fazoglo und Abyssinien aber sehr einzeln.

12. Buteo Augur, Rüpp.

Rüpp. N. Wirbelth. t. 16 und 17. - Häufig in Abyssinien mit Ausnahme der niederen Districte; auch ist er mir in W.-Abyssinien, d. h. westlich vom Tana-See blos ein einziges Mal vorgekommen. In Simehn geht er bis auf $11000^{\prime}$ Höhe. Variirt zuweilen ganz schwarzbraun.

13. Buteo rufinus, Rüpp.

R ü pp. Atl. t. 27. - Butaëtos leucurus Gmel. (?) - Einzeln in Ober-Ägypten und Nubien, häufig in Ost-Sennaar.

14. Aquila (B riss.) imperialis, Bechst.

Descr. de l'Egypt. t. 12. - $\mathrm{N}$ a u m. V. D. t. 6 u. \%. - T e m m. Pl. col.

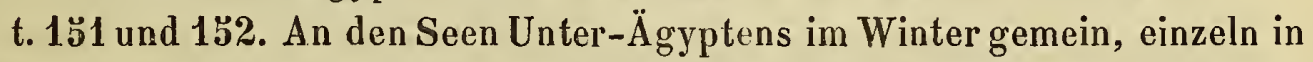
Ober-Ägypten, namentlich bei Siut, Monfaluth etc., ebenso in Abyssinien.

*15. Aquila Chrysuëtos, Linn.

A. fulva, Auct. - Naum. V. D. t. 8 und 9. - Einzeln im Herbste im peträischen Arabien gefunden, vielleicht auch in Abyssinien. (Kommt auch in der Umgebung von Tunis vor.)

* 16. Aquila naevioides, Cuv.

Cu v. Thierr. v. Voigt. S. 374. Anm. - Selten in Abyssinien und Ost-Sennaar. 
17. Aquila naevia, Linn.

Deser. de l'Egypt. t. 2, f. 1. - Na um. V.D.t. 10 u.11. - N a um. Nachtr. t. 343. - Sehr gemein an den ggrossen Seen in Unter-Ägypten. In Mä̀z und October in ganz N. O. Afrika auf der Wanderung, oft sogar in kleinen Gesellschaften bis zu 10 Stücken. Die Varietät A. clanga (Pall. et $\mathrm{Naum}$.) ist so häufig als die walı'e A. naevia.

Anm. Diese, wie die folgenden Adler-Arten heissen im Sudan "Saqr-el-armab"(_jj)

18. Aquilu rapux, T emm.

Te mm. Pl. col. t. 4ăs. - R üp p. N. Wirbelth. t. 13. - Sehr gemein südlich vom $15^{0}$ N.P. Der von $R$ üppell als A. albicans beschriebene Raubadler ist mir blos auf den Bergen von Simehn in weisslichem Kleide vorgekommen.

* 19. Aquila substriata, Heugl.

Nicht sehr selten bei Doka und Galabat (in Ost-Sennar) im Winter. Hält sich blos in der Waldregion auf.

*20. Aquila isabellina, Heugl.

Sehr einzeln auf den abyssinischen Gebirgen (Woggara).

* 21. Aquilá Bonellii, Temm.

Te mm. Pl. color. t. 288. - N a u m. V. D. Nachtr. t. 341. - Einzeln im Winter an den Seen von Unter-Ägypten.

De. Aquila pennate, Is a th.

T e mm. Pl. col. t. 33. (?) - N a u m. V. D. Nachtr. t. 343. (?) Ieh glaube kaum dass der ägyptische $/$ werg-Adler identiseh sei mit dem europäischen, da die meisten Naturforscher angeben, dass bei Letzterem die zusammengelegten Flügel die Sehwanzspitze erreichen vder überragen. In Firbung greichen die nordafrikanischen vollständig dem europaiischen, der von Te mm. (Pl. col. t. 33), Brisson (Ornith. Vol. (6. Append. p. 22, (. 1), Brehm ete. abgebildet ist; auch kommt er hierin zuweilen mit Brehm's A. minala (kaflebraun, mit weissem Schulterfleck und sogar auch ohne dem letzteren) überein, immer aber ist beim nordafrikanischen die Selıwanzspitze viel länger $\left(1 \frac{1}{2}\right.$ bis $\left.21 / 2^{\prime \prime}\right)$ als die zusammengelegten Filïgel und der Vogel überhaupt kleiner. Reslatignt sich der letzlere als neue Art, so schlage ich für ihn den Namen 1. Iongicurdul" vor und für den jedenfalls von beiden versehiedenen südalrikanischenzwerg-Adler, der, wenn ieh nicht irre, von S usemihl als A. permatu abrgebildet worden ist, A. gymnopme. Unsere A. longicrectutu ist sehr gemein vom Mär bis Oetober in den Dattelwaldungen der sogenamnten "Scherkieh" und in Unter-Ägypten überhaupt. Auf dem Striche beobachtete ich ihn lïngrs des Nils bis zun $14^{0} \mathrm{~N}$. B. 
23. Aquila (?) vulturina, D a ud.

Le Vaill. Ois. d'Afr. t. 6. - A.Verreauxii, Le ss. - Paarweise in den höheren Gebirgsgegenden von Abyssinien und Schoa. Am häufigsten traf ich ihn in den Quellenländern des Takasseh. Doch erhielt ich auch ein Exemplar vom Mareb.

24. Spizaëtos (Vi eill.) occipitalis, Daud.

$\mathrm{LeV}$ aill. Ois. d'Afr. t. 2. - Sehr gemein am Bahr el abiad, asrak und in Abyssinien. Er liebt baumreiche Chore, findet sich aber auch zuweilen in den, Steppen-Landschaften. Nicht selten habe ich Fische in seinem Magen gefunden.

*25. Spizaëtos leucostigma, He u gl.

Heugl. Beitr. t. 2. - Paarweise am Mareb, dem blauen Flusse bis Fazoglo und in den Steppen um Galabat.

* 26. Spizaëtos bellicosus, A. Smith. (?)

Ein dieser Art jedenfalls sehr nahe stehender Raubvogel, ist angeblich aus Nubien stammend, - im vice-königlichen NaturalienCabinete zu Qassr-el-aïn bei Kairo aufgestellt. Ich vermuthe, dass er durch eine der Expeditionen auf dem weissen Flusse eingesammelt wurde.

27. Circaëtos (Vieill.) brachydactylus, Wolf.

Na um. V. D. t. 15. - Im Februar, März und October häufig in kleinen Gesellschaften und einzeln auf der Wanderung längs des Nils. Überwintert in der tropischen Waldregion, wo, wie es scheint, auch einzelne zurückbleiben, indem ich z. B. Mitte Mai 1853 in Ost-Sennaar noch einen erlegte. Nach $R$ üppell's Beobachtungen kommt er auch in Arabien vor.

28. Circaëtos thoracicus, $\mathrm{Cu}$.

C. pectoralis, A. Smith. - Häufig in Abyssinien und den Bergen von Fazoglo, einzeln in 0st-Sennaar, am Bahr el abiad und in Kordofán. Ein Exemplar schoss ich im August 1852 auf der Insel Argo in DarDongola.

29. Circaëtos fasciatus, H e u g l.

Vom Herbst bis zum Frühjahr von mir im Süden von Kordofán, in den Steppen von Sennaar und lüngs des Dender und Rahad beobachtet.

30. Circaëtos cinereus, Vieill.

C. funereus R üpp. N. Wirbelth. t. 14. - Nach R üppell zufällig in Abyssinien. Ich traf diesen Vogel einmal in der Kolla von W.-Abyssinien und am blauen Flusse bei Sennaar an. Beide Individuen weichen von der Rüppell'schen Beschreibung aber darin $a b$, dass die Schwanzbinden vom reinsten Weiss und nicht „rothgrau“ gefärbt sind. 
*31. Circä̈tos zomurus, Herzog Paul v. Würtemberg.

Heugl. Beitr. t. 3. - Vom Herzog P. von Würtemberg, wenn ich nicht irre, ein Exemplar in Kamamil eingesammelt. Ich erhielt von diesem schönen Schlangenadler bis jetzt fünf Individuen vom blauen und weissen Fluss.

32. Haliaëtos (Savigny) Albicilla, L in n.

An den unterïgyptischen Seen wohnt ein weissschwänziger SeeAdler, der vielleicht vom nordischen versehieden oder wenigstens constiute klimatisehe Varietät ist. Der alte Vogel ist nümlich ganz aschgrau und die weissen Sehwanzfedern, namentlich die äussern, sind auf der Aussenfahme bräunlich bespritzt. Auch dürfte er im Allgemeinen etwas kleinere Proportionen lraben. Er heisst bei Damiette „Óqab" (عقان) oder

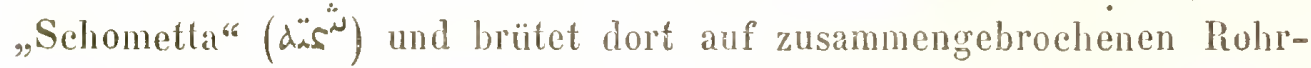
stengeln in Arumdo Donax ete. - Sollte er sich als neue Art constatiren, so selılage ich den Namen Halicëtos cinereus für ihn vor.

33. Ilaliaëtos vocifer, Le $\mathrm{V}$ a ill.

L e V aill. Ois. d'Afr. t. 4. - Hüufigam weissen und blauen Fluss, am Dender und Rahad und an den abyssinischen Gewässern. Auf arabisch „Abu Tok" (إو تولك).

34. Pundion (Savigny) Halicëtos, Linn.

Buff. Pl. enl. t. $414 .-\mathrm{N}$ a u m. V. D. t. 16. - In Winter gemein in Ägypten, an rothen Meer, auf dem Bahr el abiad, dem Atbara ete. Ich kamn nicht bestimnt angeben, ob er im Sommer hier bleibt oder wegzieht, in letzterem Falle kommt er aher früh in den Süden und zieht spät in Frülijalıre wieder fort. Ob Pandion albicollis, Brehm aus N. O. Afrika eine eigene Art oder blos Subspecies ist, kann ich nicht angeben.

33. Helolarsus (Smith) ecandalus, Daud.

L. V V a ill. Ois. dAfr. t. 7 oder II. fasciatus (?). - Ziemlich gemein in Kordofín, Semiar und Abyssinien, häufig aber in den Steppen und Gebirgen von ().-Sennar, z. B. aul Gebel-Atesch. Dort heisst er „Saqrel-arnab" oder Ilasen-Falke, in Kordofín "Satpr-el-hathím“, in Abyssinien der Ilimmels-Afle: "Hevei Sammilo" Von den von Le Vaillant beschricbenen sonderbaren liewegnngen im Fluge habe ich viel gehört, aher sie nie selbst beolachten können; wahrscheinlieh zeigt er sie blos zur Palarungs-'Zeit.

36. Inelotursus lenconotos, Herzog Panl r. Würtemberg.

Nach \&iilppel! in Semaar. Ieh zweifelte lange an der Existenz dieser zweiten Holotursus-Ant und hielt sie für Varietit des Vorhergehenden, woregen die Thatsache spricht, dass ich in November 1833 cin Pat dieser Vijgel bei Woarl-Schelay an Bahre el abiad beobachten 
konnte. Die Rückenfarbe ist vom reinsten Weiss, der Grund der Federn aber schön morgenroth. Dieselbe Art kommt auch in der Cap-Landschaft und am Senegal vor.

*37. Falco Subbuteo, Linn.

Naum. V. D. t. 26. - Einzeln im Winter in Unter-Ägypten. Ein Exemplar, dessen Identität mit dem echten Baumfalken aber noch nicht erwiesen ist, wurde im August 1852 bei Dongola erlegt.

Anm. Die Falken heissen auf arabisch „Saqr“ (صقّ) und „Bäs“ (راز), auf amharisch „Géte-Géte“.

* 38. Falco Eleonorae, Gén é.

Géné, Memor. della R. Accad. di Torino 1840. T. II. t. 1 et 2. Sus e mihl, Naturgesch. der Vögel Europa's t. 9, und t. əั3 u. əั4. Ch. Bon a p., Icon. della Fauna Ital. I. t. 24. - F. arcadicus, Lin dermayer (?) - Einzeln in Ober-Ägypten und Nubien, am blauen Fluss, häufiger auf einzelnen Inseln des rothen Meeres.

39. Falco ruficollis, Swains on.

F. ruficapillus, Herz. P aul v. Würtemberg, t. 6. - Swa inson Birds of Western Afr. t. 2. - Heugl. Beitr. t. 6, f. 1 und 2. Nicht aber $\boldsymbol{F}$. Chiquera. Le V aill. t. 30 und G o u Id Birds of Himalaya. Selten in Abyssinien, einzeln auf dem Bahr el abiad, gemein auf dem blauen Flusse von Woled Medineh südlich bis Fazoglo; vorzüglich auf Doléb-Palmen.

*40. Falco Horus, Heugl.

H e u gl. Beitr. t. 9. - Sehr selten in Ägypten und Nubien in Sandwüsten und auf kahlen Felsgebirgen.

41. Falco peregrinus, Lin $\mathrm{n}$.

Buff. Pl. enl. t. 430. - N a um. V. D. t. 24 und 25. - Häufig an den Seen Unter-Ägyptens und längs des Nils bis Nubien. Einzeln in Abyssinien. Unterscheidet sich vom europäischen dadurch, dass die Querbinden, namentlich auf der Befiederung der Tibia, nicht so dicht stehen und nicht so deutlich ausgesprochen sind. Heisst wie die drei nächstfolgenden auf arabisch „Saqr"“.

42. Falco peregrinoides, Te $\mathrm{m} \mathrm{m}$.

Te m m. Pl. color. t. 479. - Seltener in Ägypten, einzeln in Nubien und Sennaar.

*43. Falco lanarius, Linn.

$\mathrm{Naum}$. V. D. t. 23. - Einzeln in Unter-Ägypten, häufiger im Winter. Brütete im Mai 18 s̆1 auf der Pyramide des Cheops.

44. Falco cervicalis, Licht.

He u g l. Beitr. t. 4, f. 1 und 2. -F. Osiris, Herz. Pa u l v. Wür te mberg (?) - F. rubeus, A lb. Magnus (?) - Lebt vorzüglich in der 
Wüste auf kahlen Felsgebirgen, seltener auf Dattelpalmen am Nil. In Ägypten, Nubien, Kordofán und Abyssinien.

* 4\%. Falco Feldeggii, S chleg el. (?)

He u gl. Beitr. t. 4, f. 3. - Einzeln in Ägypten und Nubien.

46. Falco Aesulon, Gmel.

B uff. Pl. enl. t. 44\%. - N a u m. V. D. t. 27. - Im Winter ziemlich gemein in Ägypten. Bleibt oft bis Ende Mai.

* 47. Falco concolor, T'emm.

Te m m. Pl.color.t. 330. - S w a ins o n, Birds of Western Afr. t. 3. Falco ardosiacus, Vieill. - Ziemlich einzeln in Sennaar, Fazoglo und Abyssinien. In Amhara erhielt ich ihn am Tana-See und im TakassehQuellen-Land, in Tigréh rom Mareb. Selten längs des weissen Flusses.

48. Falco rufipes, B e s ecke.

Buff. Pl. enl. t. 431. - N a um. V. Deutschl. t.28. - St u r m, Fauna Deutschl. t. 1 und 2. - In manchen Jahren im Frühjahr und Herbst in zahlreichen Flügen, sonst einzeln längs des Nils bis Chartum beobachtet. Nach R üppell einzeln in Arabien.

49. Falco frontalis, D a ud.

L e Vaill. Ois. d'Afr. t. 35(?) - In der Sammlung Sr.k. Hoheit des Herzogs Pa u W. v. Würtemberg belindet sich ein Exemplar aus Sennaar.

ə้0. Falco (Tinnunculus, Briss.) Tinnuculus, Lin in.

Buff. Pl. enl. t. 401 und 471. - N a u m. V. D. t. 30. - Gemein in ganz N. 0. Afrika. Sehr bäufig im Winter.

* b1. Falco (Timmuculus) Alopex, Il eugl.

H e ugl. Beitr. t. 8. - Blos auf Felsgebirgen bei Doka in Ost-Sennaar, Wochni in West-Abyssinien beobachtet.

ä2. Falco (Timunculus) Cenchris, Na um.

F. Tinnusuculoides. N a tt. - N a u m.V.D. t. 29.- Stol. degli Uecelli. t. 25. - Sehr gemein in grösseren Gesellschaften im Frühjahr um Alexandria, wo er in Mauern brüten soll. Einzeln in ganz N. O. Afrika angetroften.

33. Fulco (Timunuculus) rupicola, Da ad.

L e Vaill. Ois. 1. 33. dAfr. - Häufig in ganz N. O. Afrika. R üpp.

:34. Fulco custamonotos, He $\| \mathrm{gl}$.

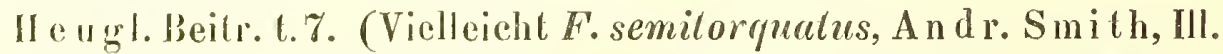
of South-Afr. 1. 1.) Sehr selten an Bahrel abiad zwischen dem 4 und $6^{0}$ N.B.

3̈). Perries (Cuv.) rpivorms, Linn.

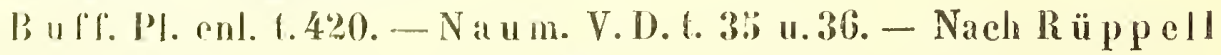

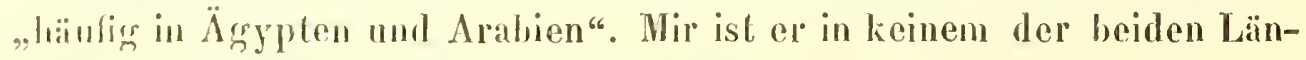
dor, ibtertaupt nie in N. O. Afrika vorgekommen. 
*56. Chelidopterix Riocourii, Vieill.

T e m m. Pl. col. t. 85. - Ich habe diesen Vogel nie selbst erlegt, aber in den Steppen von Kord̉ofán und Ost-Sennaar öfter beobachtet, so dass ich glaube, ihn ohne Anstand hier aufführen zu dürfen.

57. Elanus melanopterus, Daud.

L e $\mathrm{V}$ aill. Ois. d'Afr. t. 36 und 37. - Deser. de l'Egypt. t. 2, f. 2. Na u m. V.D. Nachtr. t. 347. - Gemein in Unter- und Ober-Agypten und Nubien. Sehr einzeln in Kordofán am weissen Fluss und am Tana-See. In $\ddot{A}$ gypten ist er Standvogel und brütet, wie es scheint, den ganzen Sommer über. (Hat dunkel karminrothe Iris.)

58. Milvus regalis $\mathrm{B}$ riss.

Buff. Pl. enl.t. 422. - N a u m.V.D. t.31.- Nach R ü p pell häufig in Unter-Ägypten.

59. Milvus ater, Lin $\mathrm{n}$.

N a u m. V. D. t. 31. - Sehr gemein in ganz N. 0. Afrika, heisst auf arabisch „Hedajeh“, wie auch der folgende.

*60. Milvus parasiticus, D a ud.

Le Vaill. Ois. d'Afr. t. 22. - Sehr häufig in ganz N. 0. Afrika.

61. Astur palumbarius, Linn.

T e m m. Pl. col.t. 495. - N a um. V. D. t. 1\%. u. 18, - Nach Dr. Rüppell „einzeln in Ägypten."

62. Meliërax (Gray) polyzonus, Rüpp.

Rüp p. N. Wirbelth. t. 15. - Gemein in Nubien, Sennaar und Abyssinien. Vielleicht gehört noch eine der vorhergehenden sehr ähnliche Art vom Bahr el abiad hierher.

63. Micronisus (Gray) Gabar, Le Vaill.

Le V aill. Ois. d'Afr. t. 33. - T e mm.Pl. col. t. 122 und 140.- Sehr häufig in Nubien, seltener in Kordofán, Sennaar und Abyssinien, auch südwärts längs des Bahr el abiad beobachtet.

64. Micronisus niger, Vieill.

Vieill. Gal. t. 22. - Wird von einigen Gelehrten als Varietät des Vorigen betrachtet, woran ich aber sehr zweifle, da ich ihn nie gemeinschaftlich mit Gabar angetroffen habe und auch die Farbe der Iris und Füsse (die sehr fahlgelb sind) verschieden ist.

lch traf ihn vorzüglich in den Waldungen von W.-Abyssinien, sehr einzeln in Sennaar und Kordofán.

65. Micronisus monogrammicus, Swa in son.

T' e m m. Pl. col.t.314. - S w a in s. Birds of W. Afr. T.I.t. 3. - Selten in der tropischen Waldregion. Ich erhielt 3 Exemplare vom Bahr el abiad, Fazoglo und Galabat an der abyssinisehen Grenze. 
66. Micronisus sphenurus, Rï p p.

M. brackydactylus $\mathrm{S}$ wain s. - M. polysonoides $\mathrm{A} . \mathrm{S}$ in ith. R ü pp. Syst. Übers. t.2. - S m it l, Ill. of South-Afr. t.11.-Dr. R ü p p e II sammelte ein Individuum dieses schönen Sperbers auf derInsel Dahlak ein. - Ich fand ihn - aber ziemlich selten - in der Kolla von W.-Abyssinien, in Galabat und am blauen Fluss. Männchen und Weibchen sind bezïglich der Färbung nicht versehieden und der Augenstern ist nicht gelb, wie Dr. Rüppell angibt, sondern lebhaft feuerroth.

*67. Hieraspiza (Ka up) mimulus, Le Vaill.

Le V a ill. Ois. d'Afr. t. 34. - Ist selten in N. 0. Afrika; ich erlielt ilm blos einmal bei Chartum, einmal in der Kolla von W.-Abyssinien und zwei Exemplare vom Mareb.

68. Hieraspiza exilis, Te mm.

H. rufiventris, A. S mith. - H. perspicillaris, R ü p p. - T e mm. Pl. col. t. 496. - Smith. Ill. of South-Afr. t. 93. - Rüpp. N. Wirbeltı. t. 18, f. 2. - Einzeln in Abyssinien und am blauen Fluss.

69. Hieraspiza fringillaria, $\mathbb{R}$ a $\mathrm{y}$.

F. Nisus, A u c t. - Buff. Pl. enl. t. 467. N a u m. V. D. t. 19 und 20. - Häufig im Winter in Ägypten. Von Dr. Rüppell auch in Arabien und Kordofán beobachtet.

70. IIieraspiza unduliventer, Rïp p.

R ü p p. N. Wirbelth. t. 18, f. 1.- Von Dr. Rüppell einzeln in den Thälern von Simehn in Abyssinien beobachtet.

81. Polyornis mipennis, Strick land.

Circus Mirllerii, He ugl. Naumannia III. t. 1. - Heugl. Beitr. t. 9, f. 1 und 2. - Häufig in Sommer an weissen und blauen Fluss, geht aber nicht weit südlich.

72. Circus (Briss.) rufus, Linn.

Bo ff. Pl. enl. t. 460. Na um. V. D. t. 37 und 38. - Gemein längs des ganzen Nilgebietes.

73. Circus umbrimus, Heugl.

Lin Exemplar am Solvat-Flusse eingesammelt.

74. Circus Maurus, 'T e mm.

Te m m. Pl. col. t. 461. - C. Lalandi S m ith, Ill. of South-Afr. t. 58. (?) - Nach $R$ ii ppell einzeln vorkommend in Sennaar und Abyssinien.

7!. Shrigiceps (Bonap.) cinerreces, Montag.

Nau 11. V. D. t. 40. - Am häufigsten von mir in Abyssinien heobachtel, einzeln auch in Ost-Sennaar und Fazoglo gefunden. In Ägypten kan er mir nie vor. 
76. Strigiceps pallidus, S ykes.

Circ. Swainsonii, A. S m ith, Ill. of South-Afr. t. 43 und 44. - N a u m. Nachtr. t. 348. - Häufig in Ägypten, Kordofán und Ost-Sennaar, seltener in Nubien. Vielleicht auch in Abyssinien.

77. Strigiceps cyanus, Linn.

Bu ff. Pl. enl. t. 459. - N aum. V. D. t. 38 und 39. - Nach Dr. Rüppell ziemlich häufig in Ägypten, Nubien und Arabien. Von mir blos einzeln in Unter-Ägypten beobachtet.

A n m. Höchst wahrscheinlich finden sich noch verschiedene andere Weihen - Arten, wie C. ranivorus etc. in N. O. Afrika und am weissen Fluss, doch kann ich bis jetzt nichts Sicheres hierüber angeben.

78. Polyboroides typicus, $\mathrm{Sm}$ ith.

F. gymnogenys, Temm. Pl. col. t. 307. - Gymnogenys madagascariensis, Les son. - A. Smith, Ill. of South-Afr. t. 81. - Ist selten und findet sich blos in waldigen Gegenden oder an Flussufern, die hohe Bäume in ihrer Nähe haben. Ich erhielt ihn im Monat Juni und Juli von El-Afun bei Chartum und von der Gegend um Sennaar, Dr. Rüppell aus Schoa.

79. Gypogeranus (Illig.) serpentarius, Lin $n$.

Serpentarius reptilivorus, D a d. - B uff. Pl. enl. t. 721. - L e V a ill. Ois. d'Afr. t. 25.-Zur Regenzeit häufig in Abyssinien, einzelner in Sennaar u. Kordofán. Brütete im September 1853 unweit Chartum. Im November 1853 traf ich ihn nicht selten in Kordofán, namentlich bei Gebel Kohn und Gebel Bedji, so dass ich in zwei Tagen sechs Stücke lebend einfangen konnte. Die Jagd auf ihn wird zu Pferde gemacht und der Vogel so lange verfolgt, bis er nicht mehr zu fliegen im Stande ist. Auf dem Scherk-el-akaba

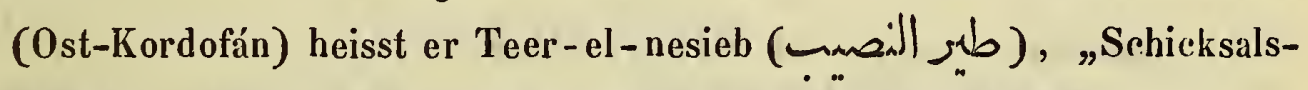
Vogel.“- Nicht ganz mitRecht führt er den Namen reptilivorus, da er, so viel ich beobachten konnte, mehr von Säugethieren bis zur Grösse von jungen Antilopen und von Schildkröten als anderen Reptilien lebt.

A n m. Zweifelsohne kommen in N.O. Afrika auch manche Falken-Arten vor, die mir entgangen sind. So viel ich mich noch erinnere, sind z. B. in der Sammlung des Herzogs P. v. Wür t e m berg, als von hier stammend noch folgende Species aufgestellt: Milvus Isuroides und M. aethiopicus, Herz. Paul v. W., - Buteo Tachardus, S h a w, - Circus chrysocomus, Herz. P a ul.v. W.,-C. ranivorus, D a ud., - C. Acoli. L e Vaill., - verschiedene Adler ete. etc. die ich leider zu vergleichen nicht Gelegenheit hatte.

\section{STRIGIDAE.}

Die grossen Eulen heissen auf arabisch „Buma“ (dog), die kleinen „Oın-queq"

$$
\text { (أو }
$$

80. Athene (Boje) meridionalis, Risso.

Strix passerina, Lin n. - Sehr häufig in ganz N.O. Afrika. Fliegt häufig bei Tage auf Raub aus. 
* 81. Athene occipitalis, I emm.

T e mm. Pl. col.t. 34. - Einzeln südlich vom $15^{0}$ N. B.

82. Athene pusilla, Lath.

L e Vaill. Ois. d'Afr. t. 46. - N a n m. V. D. t. 43, f. 1, 2. - Nach IR üp p ell einzeln in Sennaar und Abyssinien. Vielleicht verwechselt er diese Art mit der vorhergehenden.

S3. Scops (Savigny) vulgaris, Cuv.

Strix Scops, Linn. - B u f f. Pl. enl. t. 436 . - N a u m. V.D.t. 43 , f. 3. - Einzeln und paarweise in ganz N. 0. Afrika in waldigen Gegenden und Gärten.

84. Bubo maximus, Sib b.

N a u m. V. D. t. 44. - Einzeln im Winter in Unter-Ägypten.

85. Bubo Ascalaphus, Savigny.

Descr.de l'Egypt.t. 3, f. 2.-- Te m m. Pl. col.t. 5\%.--Paarweise und in kleinen Gesellschaften in Ober-Ägypten und Nubien. (Ich habe ihn arch von Tripolis erhalten.)

86. Bubo crepensis, Da ud.

Smith, III. of South-Afr. t. 70. - In Scho\%.

8\%. Bubo lacteus, 'Temm.

T e $\mathrm{m}$ m. Pl. col. t. 4 . - Hïufig in den tropischen Wald-Regionen, vorzüglich längs des blauen und weissen Flusses. In Abyssinien bis $8000 \mathrm{~F}$. hoch angetroffen. Sieht auch sehr gut bei Tag und lässt sich leicht zähmen.

* 88. Aegolius (K eys. et B las.) Otus, Linn.

Na um. V. D. t. 45, f. 1. - Nicht selten im Winter in Ägypten.

89. Aegolines montromes, He ugl.

Nicht selten in Waldpartien und namentlich auf Colqual-Euphorbien auf den Gebirgen von Woggara und Simehn bis zu einer Ilöhe von $11.000 \mathrm{~F}$.

90. Aegolims "ricamus, Linn.

Te m m. Pl. col. t. 50. - Nicht eben häufig in Waldpartien, süalich voin $18^{0}$ N. B.

7 91. Acyolius rbyssinicus, Guérin.

liei Gondar.

92. Argolines leucolis, 'Tem m.

T'e min. Pl. col.1.16. - Nicht selten südlich rom $18^{\circ}$ N. B.

9:3. Aegolims copenesis, A. Simith.

Smith, Ill. of South-Afr. t. 67. - In Schou. 
94. Aegolius brachyotus, Forst.

B uf f. Pl. enl. t. 438. - Naum. V. D. t. 4马, f. 2. - Nicht selten einzeln und in grösseren Gesellschaften in Nubien, Ägypten und Abyssinien. Ich habe ihn blos im Winter beobachten können und traf ihn immer nur in Büschen in der Wüste.

95. Strix flammea, Linn.

Buff. Pl. enl. t. 440. - Naum. V. D. t. 4\% - In allen ihren Varietäten st die Schleiereule nicht selten in ganz N. 0. A firika. In Ägypten bewohnt sie alte Gebäude und in Abyssinien traf ich sie nicht selten in Wäldern und auf Hochbäumen. Im Winter 1833-54 erhielt ich sogar mehrere Exemplare vom Berge Be lini a, am Bahr el abiad ( $3^{0}$ N. B.).

\section{ORDNUNG. PASSERES (SPERLINGVÖGEL).}

\section{Fissirostres.}

\section{A. CA PR INI UI, GID A E.}

96. Caprimulgus europaeus, Linn.

Na u m. V. D. t. 148. - Im Winter in N. 0. Afrika.

97. Caprimulgus infuscatus, Rüp p.

R ü p p. Atl. t. 6. - Nicht selten in Nubien und Kordofan.

98. Caprimulgus tristigma, Rüpp.

Rüpp. Syst. Übers. t. 3. - Einzeln im südlichen Abyssinien. Rüppell.

99. Caprimulgus poliocephalus, Rüpp.

$R$ ü p. Syst. Übers. t. 4. - Einzeln in den nordöstlichen Thälern von Abyssinien. R üp pell.

100. Caprimulgus isabellinus, Temm.

Te m m. Pl. col. t. 379. - He ugl. Beitr. t. 40. Das Ei. - Paarweise und zuweilen in grossen Gesellschaften bis zu 40 Stücken in Steppen und Mimosenwäldern. In Ägypten habe ich ihn auf der Wanderung im Monat April und Mai gefunden; in Nubien, vorzüglich auf den Inseln bei Argo, brüteten einige Paare im August und September 1834. Auch soll er in Abyssinien vorkommen.

101. Caprimulgus eximius, Rüpp.

T e mm. Pl. col. t. 398. — In Sennaar, überall einzeln.

*102. Caprimulgus ruficollis, Natterer (?)

Oder wenigstens eine diesem sehr nahe stehende Species. Häufig in Tigréh und aun Mareb in Abyssinien.

103. Scotornis (Swain son) climacturus, Vieill.

Vieill. Gal. t. 122. - Häufig in Sennaar, Kordofan und S.-Nubien. 
10皇. Hacrodipterye (Swainson) longipennis, Shaw.

S wainson, Birds of Western-Afr. V.II. t. ๖. -- Nach Rüppell einzehn im östichen Abyssinien. Im Monat December und Januar sehr gemein in Ost-Sennaar, Gababat und Wochni, wo ieh aber schon im April und Wai keinen mehr antraf. Einzeh im sülllichen Sennaar bei Rosseres, in Fazoglo am weissen Flusse und in Kordofan. Heisst auf arabiseh Abu-gennáh-

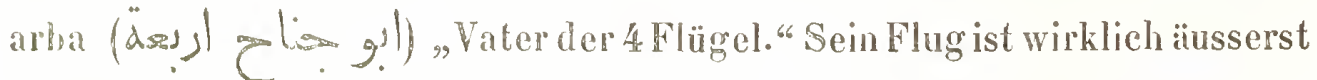
sonderlar und man glaubt einen grösseren und zwei ihn verfolgende und auf ihn stossende kleinere Vögel zu erblicken. Am häufigsten erlegten wir ihn in der Kolla an unsern Wachfeuern, die oft die ganze Nacht von diesen Thieren unsehwärm waren.

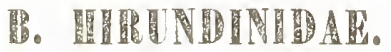

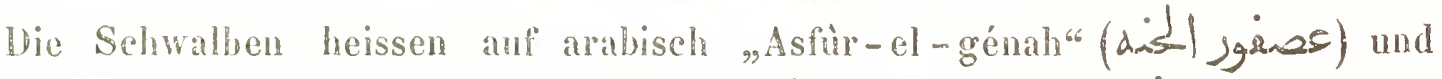
"Chothif" (خاف)

103. Cypselus (IIIig) Apus, Linn.

N aum. V. D. t. 14\%, f. $2-$ C. murimes, ti hre nb. (?). - Buff.

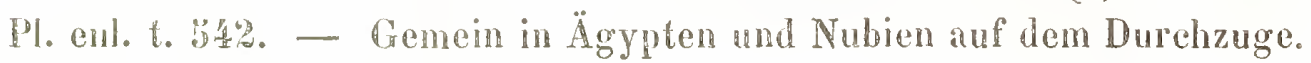

106. Cypselus pouppollii, He 1 igl.

C. abyssimicus, Lieht. (?) - In Abyssinien und am blauen Elusse in kleinen Gesellschaften.

10\%. Cypselus Mellou, Tinn.

C. alpims, Seop. - Naum. V. D. t. 14\%, f. 1. - An den Felsgehirgen $\ddot{\Lambda} g y p t e n s$ in Frïhjahr auf dem Durehzuge.

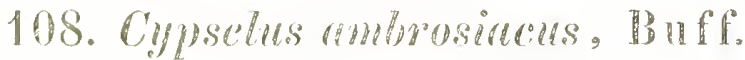

T'mm. 1\%. col. 1. 460, f. 2. - In Nubien und Sennaar, nach If ii ppell auch in Ägypten.

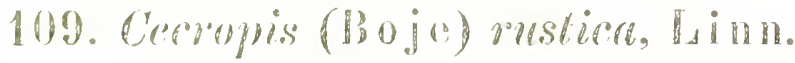

Hirmedo domestere, I'all. - Im Winter in ganz N. O. Afrika.

* 110. Cecropies alpestris, Pall.

JHirundo vufulu, T' e m u. - In Nubien und West-Abyssinien.

111. Cecroplis biocourai, Sivigny.

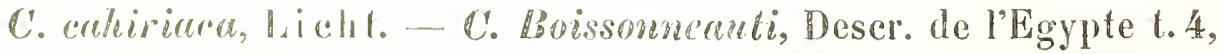
f. 4. - In $\ddot{A}$ gyputen Standvogel.

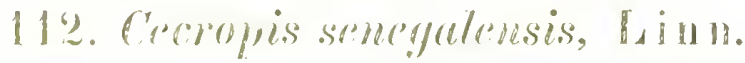

Swatinson, Brids of Western Afr. V. II. 1. 6. - Häufig in KorJofin und ann Tiula-see in Mbyssinien.

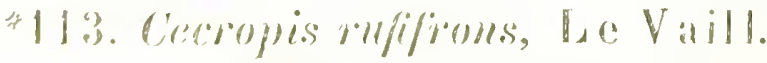

Standvorel in Semnatar and S. Nubien.

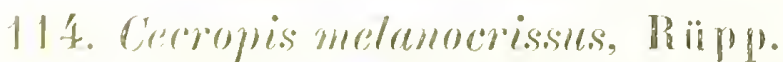

R ïp p. Syst. Übers. 1. מ. - M. Gordoni Jard. (?) In den abyssinisehen Gebirgsthïlem nicht selten, aber gewöhnlich einzeln oder paar- 
weise beisammen. Geht bis 10-11.000 F. hoch und lässt zuweilen einen von dem aller mir bekannten Schwalben abweichenden Gesang in den Lüften hören.

115. Cecropis striolata, Rüp p.

Rüp p. Syst. Übers. t. 6. - C. abyssinica, Guerin. - In kleinen Gesellschaften in den abyssinischen Gebirgs- und Hochländern.

116. Cecropis filicaudata, Lath.

L ath. Gen. Hist. of Birds t. 113. - Nicht selten in Kordofan, Sennaar und Abyssinien.

117. Cotyle (Boje) torquata, Linn.

Buff. Pl. enl. t. 723, f. 1. - Von Dr. Rüppell in der abyssinischen Provinz Barakit beobachtet; ich erhielt sie häufig vom Mareb.

118. Cotyle paludibula, Le V aill.

Le V a ill. Ois. d'Afr. t. 246, f. 2. - In Nubien, Sennaar, Abyssinien und auf dem weissen Fluss.

119. Cotyle riparia, Lin $\mathrm{n}$.

B u ff. Pl. enl. t. 543. - Nau m. V. D. t. 146, f. 3 und 4. - Im ganzen bekannten Nil-Gebiete.

120. Cotyle rupestris, Scop.

$\mathrm{N}$ a um. V. D. t. 146, f. 1. - Häufig in Ägypten, Nubien und Abyssinien in Felsgebirgen und Ruinen. Standvogel.

121. Chelidon (?) (Boje) pristoptera, Rüpp.

Von Dr. Rüppell während der Regenzeit häufig in Simehn beobachtet. Ich traf diese Schwalbe blos einmal, aber in grosser Gesellschaft in den Waldungen der westlichen Kolla-Länder (Provinz Dagossa) und erhielt einige Exemplare aus Tigréh (am Amba-Sea). Wahrscheinlich auch am Mareb.

122. Chelidon urbica. Linn.

Im Winter im Nil-Gebiet.

\section{CORACIANAR.}

123. Eurystomus (Vieill.) orientalis, Linn.

Colaris afra, Lath. (?)-C. madagascariensis, Gmel. (?) - L e V a ill. Ois. de Parad. I.t. 35. - Einzeln in Abyssinien, Sennaar, Fazoglo und Kordofan.

124. Coracias garrula, Linn.

Buff. Pl. enl. t. 486. - $\mathrm{N}$ a um. V. D. t. 60. - Im Winter in Ägypten, Nubien und Arabien. Ende April auf dem Wiederstrich in UnterÄgypten sehr häufig.

125. Coracias abyssinica, Gmel.

C. senegalensis? - B uff. Pl. enl. t. 626. - Gemein südlich vom $20^{0}$ N. B. 
126. Conacias Levaillantii, Te mm.

Le Vaill. Ois. de Par. I. t. 29. - Nicht seiten in Kordofan, Sennatar und Abyssinien von der Kolla abwïrts.

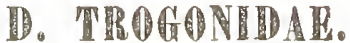

12\%. Apaloderma. (Swainsou) Nrevina, Vieill.

Le Vaill. Ois. d'Afr. t. 28: - Selten am Mareb, in der Kolla, der Provinz Wochni und in Fazoglo.

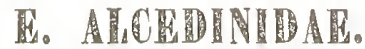

128. Ifralcyon (Swainson) semicoemlea, Forskäl.

船 ï p. N. Wirbelth. t. 21, f. 1. - Häufig in Abyssinien.

129. Haleyon comeropheraga, Lath.

B uff. Pl. enl. t. 334 . - Einzeln aul dem blauen und weissen Flusse.

130. Halcyon chelicuti, Stanley.

R ï p p. Atl. t. 28, f. b. - Iläufig, aber meist blos einzeln, in Abyssinien und Sennaar auf Buschwerk und Bäumen, oft in Mitte von Waldungen. Nicht auf höteren Gebirgen, wenigstens von mir nicht über 6000 Fuss hoch beobachtet.

181. Ceryle (Poje) mulis, timn.

Bu ff. Pl. enl. t. \%16. - Gemein in ganz N. 0. Afrika an fliessenden Gewïssern.

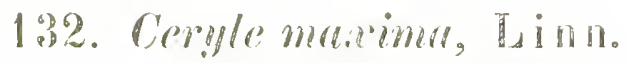

Pbufr. Pl. cnl. t.689. - Gemein am Dender, Rahad, der Gandoa, dem Takasselt, zuweilen an ganz unbedeutenden Choren und Wassergläbrn. - Vinzeln anch in Fazogito。

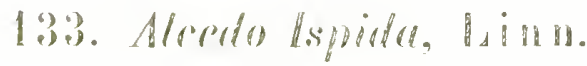

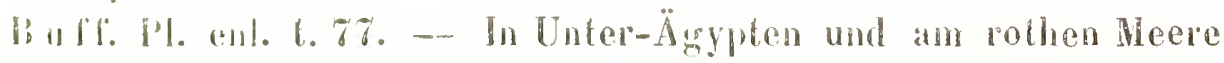
won mir l, wos in Winter heobachtet.

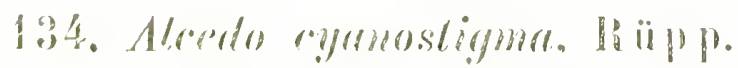

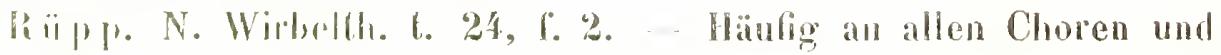

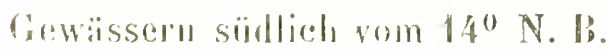

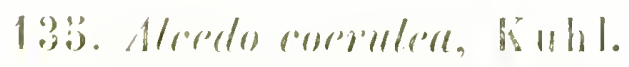

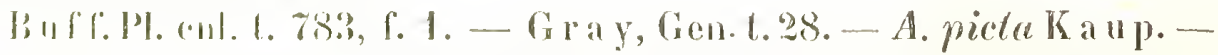

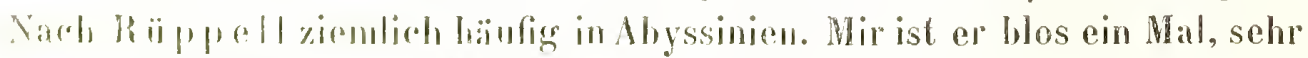
ferm non firwissem, bei fiebel Woad Dambellie in Ost-Sennaar vorgekommen, als er an einem ghöhend heissen Tago lleuschecken jagte, mit demen sein Vagen angelïllt watr. In Sürl-Sennatr sammelten ihn meine Jitiger anch einige Male ein. 
136. Alcedo semitorquata, S wain son.

Rüpp. Syst. Übers. t. \% - Nach R üppell in Schoa. Ich fand ihn an allen Wildbächen zwischen Simehn und 0.-Sennaar ziemlich häufig.

Anm. Am rothen Meere und in Abyssinien dürften sich noch manche hierher gehörige Arten vorfinden; an den Ufern des ersteren fiel mir ein dem Alcedo collaris in Färbung sehr ähnlicher Eisvogel auf, und am Takasseh traf ich eine sehr kleine und eine andere weit grössere Art, dereu Hauptfarbe rostroth bis kastanienbraun zu sein schien. (A. madagascariensis?) Nach Versicherung des Herrn Dr. Rüppell findet sich auch A. capensis in Schoa.

\section{F. MEROPIDAE:}

137. Merops Apiaster, Lin n.

Le Vaill. Prom. t. 1. - Im März und April und im Herbst als Zugvogel in ganz N. 0. Afrika; doch babe ich ihn merkwürdiger Weise wie den folgenden auch zuweilen in Sommer in Sennaar angetroffen.

138. Merops superciliosus, Lath.

Merops persicus, Savign. - Merops Savignii, Swains. Birds of W. Afr. t. 7. - Le Vaill. Prom. t. 6.

*139. Merops Cuvieri, Li cht.

M. albicollis, Vieill. - Le Vaill. Prom. t. 9. - Das ganze Jahr. aber einzelner, in Kordofan und Sennaar.

140. Merops viridis, L ath.

Le Vaill. Prom. t. 10. - M.viridissimus, $\mathrm{S}$ w a ins. (?) - Häufig als Standvogel in ganz N. 0. Afrika südlich vom $28^{\circ}$ N. B.

141. Merops coeruleocephalus, Lath.

IM. nubicus G mel. - Buff. Pl. enl. t. 356, f. 2. - Swain s. Birds of West. Afr. II. t. 9. - Le Vaill. Prom. t. 3. - H e u g l. Beitr. t. 40 : das Ei. - In grossen Flügen und als Standvogel am blauen und weissen Flusse und in Ost-Sennaar. Nach Rüppell auch in Abyssinien und Kordofan. - Brütet in grossen Colonien in selbstgegrabenen Löchern auf ebener Erde in den Ländern der Kitsch-Neger im März.

142. Merops erythropterus, Gmel.

Merops Lafresnayi, Guérin. (?) - M. minutus, Vi eill. (?) Lath. Gen. history of Birds t. 70 . - Südlich vom $16^{0} \mathrm{~N}$. B. nicht selten.

143. Merops variegatus, Vieill.

Le Vaill. Prom. t. 7. - Im Januar, Februar und März häufig um Gondar angetroffen. Im Sommer am Mareb und in Tigréh. 
144. Merops Bullockii, L e Vaill.

Le Vaill. Prom. t. 20. - In der Kolla, namentlich am West-Abfall gemein bis nach Galabat. Einzeln in Sennaar und Fazoglo.

\section{Tenuirostres.}

\section{A. UTUPIDAE.}

140. Upupa Epops, Linn.

In ganz N. O. Afrika, häufiger im Winter. Arabiseh „Hud-Hud“ ( 2010 )

146. Promerops (Briss.) erythrorhynchus, Cuv.

L e V a ill. Prom. t. 1 u. 2. - Hïufig südlich vom $15^{0}$ N. B. Brütet in hohlen Bäumen.

147. Promerops cyanomelas, $\mathbb{C} u \mathrm{v}$.

Le V aill. Prom. t. g u. 6. - Wie der Vorhergehende.

148. Promerops minor, Rüpp.

Rüp p. Syst. Übers. t. 8. - In Sehoa.

*149. Promerops icterorhynchus, Heugl.

Pr. pusillus, Swains. (?) - Einzeln im Lande der Bari-Neger zwischen dem 4 . und $6^{0} \mathrm{~N}$. B.

A n m. Prom. senegalensis, Vieill. - L e V a ill. Prom. t. 4. - soll nach $\mathrm{Strickland}$ in Kordofan vorkommen.

\section{B. NEC TARINDDAE。}

130. Nectarinia (Illig.) fumosu, Vieill.

Vie ill. Ois. dor. If. t. $3 \%$ u. 38. - In Abyssinien.

131. Nectarivia pulchellu, Vieill.

Je V a ill. Ois. d'Afr. 1. 293. 1. - Gemein in S.-Nubien, Sennaar und Kordofan.

1:32. Necturinia Tacazse, Stanley.

Rï̈p. N. Wirbelth. t. 31, f. 3. - Gemein in Abyssinien. Geht ïl,er 10,000' F'. hoch. In der Kolla von West-Abyssinien nieht beobachtet.

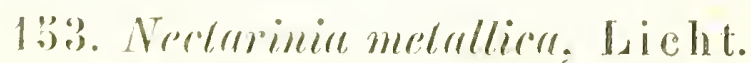

R ïрp. Atl. 1. \%. - Gemein südlieh vom $24^{0}$ N. B.

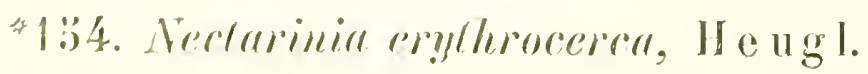

Hengl. Beitr. t. 10, f. 2. - Auf dem Bahr el abiad südlich vom 8" N. In nicht selten. 
*15้. Nectarinia porphyreocephala, H e u g l.

H e u g l. Beitr. t. 10, f. 1. - N. purpurata, Ill. (?) - Einzeln im südöstlichen Abyssinien.

156. Nectarinia affinis, Rüp p.

R üpp. N. Wirbelth. t. 31, f. 1. - Einzeln in Abyssinien, häufig in Kordofan.

157. Nectarinia gularis, Rü p p.

Rüpp. N. Wirbelth. t. 31, f. 2. - Nach R üppell in Kordofan.

158. Nectarinia habyssinica, Ehr enb.

Ehr enb. Symb. Aves. t. 4. - In Abyssinien.

159. Nectarinia cruentata, R üpp.

R üpp. Syst. Übers. t. 9. - Häufig in Abyssinien bis zu 10,000'F. Höhe, einzeln am Bahr el abiad, südlich vom $8^{0}$ N. B.

\section{CERTHINAE.}

160. Tichodroma (Illig.) muraria, Lin n.

Nach R üppell in Ägypten und Abyssinien. Von nir nie beobachtet.

3. Canori.

\section{A. S Y I V I D A E.}

1. Malurinae.

161. Oligura micrura, Rü p p.

Rü p p.N. Wirbelth. t. 41,f.1.-Oligocercus micrurus, $\mathrm{C}$ a b a n is. In Nubien, Kordofan, Ost-Sennaar und den Kolla-Ländern von W.-Abyssinien, findet sich auch am weissen und zuverlässig längs des ganzen blauen Flusses. Im Benehmen und Lockton hat dieses zierliche Vögelchen viel Ähnlichkeit mit Sitta europaea, der es auch bezüglich der Farbenvertheilung nahe kommt.

162. Cisticola (L ess.) schönicola, Bo nap.

Sylv. cisticola, T e m m. - Häufig in Arabien, Ägypten und Nubien. Standvogel.

* 163. Cisticola ferruginea, $\mathrm{Heugl}$.

H e u gl. Beitr. t. 12, fig. 2. - Blos in den Quellenländern des Rahad beobachtet.

164. Cisticola (?) lugubris, Rüpp.

Rü p p. Syst. Übers. t. 11. - Einzeln in Abyssinien.

165. Cisticola (?) erythrogenys, Rü p p.

R üp p. Syst. Übers. t. 12. - Einzeln in Abyssinien.

*166. Cisticola (?) flaveola, H e u gl.

H e u gl. Beitr.t.11, f.1. - Einzeln in Tigréh im östlichen Abyssinien. 
167. Cysticola (?) mystacea, Rïp.

Rüp p. Syst. Übers. t. 10. - Aus der Umgegend ron Gondar.

168. Cysticola(?) mufrons, Rüpp.

Rüpp. N. Wirbelth. t. 41, f. 1. - Häufig an der abyssinischen Küste.

169. Cysticola (?) mficeps, Rüpp.

R üp p.Atl. t. 36, f. a.-H Hüufig in Kordofan, Sennaar und Aby̦ssinien.

*180. Drymoica ( $\$$ wainson) leucopygia, Heugl.

In Ost-Sennaar und Abrssinien.

131. Drymoica inquieta, Rüpp.

R ̈̈pp. Atl. t. 36, f. b. - Hüufig in niedrigem Gestrüppe bis auf ${ }_{4} 000 \mathrm{~F}$. Höhe im peträischen Arabien.

172. Drymoica robuste, Rüp p.

Ruipp. Sỵst. Übers. t. 13. - Cm Gondar und in der Kolla von W.-Abrssinien. Dr. R üp pell hat sie aus Schoa erhalten.

*183. Drymoica Halsacii, Heugl.

Im Gebiete der Kitsch-Neger zwischen dem i- $9^{0} \mathrm{~N}$. B.

*174. Drymoica cantans, Heugl.

Auf den Hochgebirgen ron Simehn.

*17ö. Drymoira marginatis, He ugl.

Am Bahr el abiad zwischen dem $6-9^{0}$ N. B.

176. Drymoica bisomure. Heug].

He ugl. Beitr. t. 12, f. 1. - Blos in der Prorinz Simehn in dichten, wasserreichen Schluchten bis $10.000 \mathrm{~F}$. Höhe angetroffen. (Kommt whescheinlich auch bei Gondar ror.)

19\%. Drymoica pulchella, RüpT.

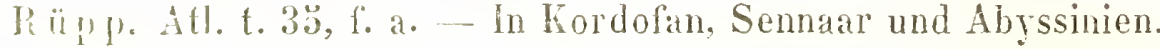

178. Drymoira groncilis. Piupp.

IR ü p p. All. t. 2, f. b. - Häulig in Äggpten und Nubien.

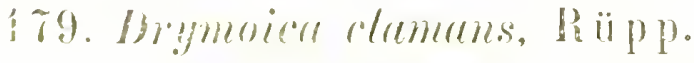

Rü̈p. . Itl. t. 2. f. a. - In südlichen Nubien, Kordofan und Sennaar.

2. Sylvinar.

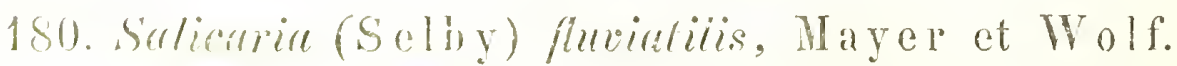

Hrescr. de l'Eg. 1.13. - Naum. V. D. t. 83, f.1. - In Unter-Ägypten.

181. Sulinaria arumdinacen. B riss.

Nanm. V. I. 1. 81, f. 2. - In Ägypten, Nubien und Arabien.

182. Serlicurice pullide, Ehrenb.

If eng lo licitr. 1. 40, das E.i. - In ganz N. O. Afrika, das ganze Jahle hiorlurelit. 
183. Salicaria turdoides, Mayer.

B uff. Pl. enl. t. 513. - Naum. V. D. t. 81, f. 1. - In UnterÄgypten und Arabien im Winter.

184. Salicaria stentoria, Cab.

In Arabien und Ägypten.

185. Salicaria palustris, Bechst.

Na um. V. D. t. 81 , f. 3. - Nach Rüppell in Ägypten.

186. Salicaria phragmitis, B e chst.

Naum. V. D. t. 82, f. 1. - Im Winter in Ägypten und Nubien. Ich erhielt sogar Exemplare vom Sobat.

187. Salicaria aquatica, Lath.

Na u m. V. D. t. 82, f. 4 und 5. - Im Winter in Delta.

188. Salicaria (?) cinnamomea, Rüpp.

R ü p. N. W. t. 42, f. 1. - In Abyssinien.

An m. Salicaria (?) languida, E h renb. aus Ober-Ȧgypten und Nubien ist mir nicht bekannt. - Ausserdem sollen noch einige hierher gehörige Sänger in N. 0. Afrika vorkommen, wie S. Luscinioides, S avi. ete.

*189. Ficedula (Ko ch) Hypolais, Linn.

Na um. V. D. t. 80, f. 1. - Im Frühjahr in Ägypten.

190. Ficedula sibilatrix, B e chst.

Naum. V. D. t. 80, f. 2. - Im Winter in Ägypten.

191. Fricedula Trochilus, Linn.

F. fitis, Koch. - Nau m. V. D. t. 80, f. 3. - In Ägypten und Nubien im Winter.

192. Ficedula Bonellii, Vieill.

S. Nattereri, T emm. - In ganz N. 0. Afrika.

193. Ficedula rufa, Lath.

Na u m. V. D. t. 80, f. 4. - Im Winter in ganz N. 0. Afrika.

194. Ficedula umbrovirens, R ü p p.

Rüpp. N. W. S. 112. - In wärmeren Gegenden Abyssiniens. Ieh erhielt sie öfters vom Mareb.

*195. Ficedula elegans, He u $\mathrm{l}$ l.

Heugl. Beitr. t. 13, fig. 1. - In den Thälern der westlichen Kolla-Länder.

196. Sincopta (C a b.) brevicaudata, R ü p p.

Rüpp. Atl. t. 35, f. b. - In Kordofan, Sennaar und Fazoglo. 
*19\%. Orthotomus (Horsfield.) (?) clamans, Heugl.

H eugl. Beitr. t. 13, fig. 2. - In Abyssinien, Sennaar und Kordofan. - Eine ähnliche, aber von ihr verschiedene Art - wenn ich nicht irre - aus Fazoglo, steht in der Sammlung des Herzogs Paul von Würtemberg.

*19S. Orthotomus Salvadorae, Herzog Paul v. Würtemberg.

Von Fazoglo. (Gleicht́ dem Vorhergehenden, hat aber roströthliche Stirne und gelblich fleischfarbenen Schnabel, mit schwärzlicher Firste gegen die Spitze zu).

199. Zosterops (Gould) madagascariensis, Lath.

Le Vaill. Ois. d'Afr. t. 132. - In Abyssinien nicht selten.

*200. Zosterops euryophthatmos, He u g l.

Heugl. Beitr. t. 13, fig. 3. - Blos ein Paar auf den Hochgebirgen von Simehn bei Debr Eski beobachtet.

*201. Sylvia (Pennant) subalpina, B on elli.

S. leucopogon, Mayer. - S.passerina, T emm. - Im März und April in Unter- ̈̈gypten.

*202. Sylvia provinciatis, J. F. Grmel.

Wie die Vorige.

203. Syluia melanocephala, Lath.

Im Winter hä̈ufig in Arabien, im Frühjahr in Ägypten und Nubien beobachtet.

204. Sylvir Orpher, Temm.

N a um. V. D. t. 76, f. 3 und 4. - Im Herbst in Ägypten beobachtet.

205. Sylrive Curmer, Lath.

N a u m. V. D. t. $7 \%$, f. 1. - In Herbst und Frühjahr in Ägypten, Arabien und Nubien.

206. Sylleia atricapilla, Bris is.

Na um. V. D. 1. $7 \%$, f. 2 und 3. - Im Frühjahr in Ägypten, Nubien und Arabien.

207. Sylvia migrioupilla, Cab.

Nach Cab an is in N. O. Afrika.

*20S. Syllier ruficapilla, La and beek (?)

Der vorigen :ihnlich, o und 9 mit rostrothem Seheitel. - In Abyssinien (Simein und bei Massaua) und in Nubien.

20)9. Sylrir lï̈ppellii, 'I' emm.

S. copistrula, Rëip p. Atl. t. 19. - Im März und April häufig in Ägypten und Arabien. 
210. Sylvia cinerea, Bris s.

N a um. V. D. t. 78, f. 1 und 2. - Im Winter in ganz N. 0. Afrika. *211. Sylvia hortensis, P ennant.

N a u m. V. D. t. 78, f. 3. - Im Frühjahr in Ägypten.

*212. Sylvia Nisoria, B echst.

Na um. V. D. t. 76, f. 1 und 2. - Im October und April in Nubien und Sennaar beobachtet.

213. Sylvia crassirostris, R üpp.

R üpp. Atl. t. 33. - In Sennaar und Kordofan, am weissen Flusse. Überall einzeln. - Ist vielleicht identisch mit $\mathbf{S}$. olivetorum.

214. Sylvia chocolatina, R üp p.

R ü pp. Syst. Übers. t. 14. - Abyssinien und Schoa.

215. Sylvia lugens, Rüpp.

R üpp. N. Wirbelth. t. 42, f. 2. - In Abyssinien.

*216. Lusciola (Ke y s. et B las.) Philomela, B e ch st.

Na u m. V. D. t. 74, f. 1. - Im Frühjahr in Ägypten.

217. Lusciola Luscinia, Li nn.

Na um. V. D. t. 74., f. 2. - In Ägypten, Nubien und Arabien im März, April und September.

218. Aedon galactodes, T e m m.

In ganz N. 0. Afrika, scheint im Winter südwärts zu wandern.

219. Aedon minor, Cab.

Nach Cabanis in Abyssinien.

220. Aedon leucopterus, Rüp p.

Rüp p. Syst. Übers. t. 15. - In Schoa.

221. Aedon abyssinicus, Rüp p.

Drymophila abyssinica, R üpp. N. W. t. 40, fig. 2. - In den abyssinischen Gebirgsthälern.

222. Cyanecula (Brehm) suecica, Linn.

Na u m. V. D. t. 75. - Häufig im Winter in Ägypten, Nubien, Arabien und Abyssinien, zuweilen mitten in der Wüste.

223. Erythacus (Swainson) Rubecula, Linn.

Naum. V. D. t. 75. - Im Februar und März in Unter-Ägypten.

224. Ruticilla (Brehm) Phönicurus, Linn.

$\mathrm{N}$ a u m. V. D. t. 79, f. 1 und 2. - In ganz N. 0. Afrika, vielleicht den Sommer über in Abyssinien, wo ich ihn im April noch häufig beobachtete.

225. Ruticilla Tithys, Scop.

N aum. V. D. t. 79, f. 3 und 4. - Im Frühjahr in N.-Ägypten. 
3. Saxicolinae.

226. Suxicola (Bechst.) leucura, G mel.

S. cachinans, Temm. Deser. de l'Egypte. Ois. t. 5, f. 1. Hïufig in Ägypten, Nubien und Arabien. - Gewöhnlich haben die Weihchen weisse Kopfplatten, doch fand ich öfter auch Münnchen mit dieser Zeichnung.

227. Saxicola Monacha, Rüpp.

Te m m. Pl. col. 339. 1. - Nach R üppell zufällig in Nubien.

*28. Saxicola syenitica, Heugl.

Heugl. Beitr. t. 14. - Einzeln in Ober-Äggpten.

229. Saricola lugubris, Rüp p.

R ï p p. N. Wirbelth. t. 28, f. 1. - Häufig in Abyssinien.

230. Sraxicoln melaenu, $\mathrm{R}$ ü $\mathrm{p}$ p.

R ü p p. N. Wirbelth. t. 28, f. 2. - Häufig in Abyssinien, namentlich in den Gebirgen von Woggara und Simehn.

231. Suxicola albifions, Rüp p.

Rüp p. Syst. Übers. t. 1\%. - S. frontalis, Swains.(?) - Häufig im Tilkasseh-Quellenland.

232. Saxicoln lugens, Li cht.

In Ägypten, Nubien und Arabien.

233. Sarcicola isabellina, Rüp p.

\$. saltation. Nénestriés. - Häulig in ganz N. 0 Afrika.

* 234. Saricola firmuginea, Heugl.

In Alsyssinien.

230. Saxicola pallida, R ï p p.

R ïpp. All. 4. 34, f. a. - Nach R üppell loüufig in Nubien.

236. Saxienla Oenumber Be ehst.

Buff. Pl. enl. 1. 434. - Naum. 1. 89, f. 1. - In ganz N. O. Afrika, wie es scheint alser bos im lrühjahr.

237. Saricola Stapasina, Gimel.

N a u m. V. I).1.90, f. 1 mud 2 . - Bäufig in N. 0. Afrika, in Ägypten blos in . Hir\% und spril. Feh halle von ihr für versehieden:

238. Saricola andila, Temm.

Ebendaselbst.

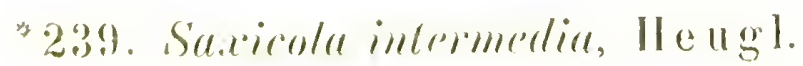

Häulig in Alygsinien. 
240. Saxicola deserti, R ü p p.

T e mm. Pl. col. t.359, f. 2. - In Ägypten und Nubien nicht selten.

241. Saxicola sordida, R üp p.

R üpp. N. Wirbelth. t. 26, f. 2. - Häufig in den Gebirgen von Waggara und Simehn in Abyssinien.

242. Saxicola rufocinerea, Rüpp.

Rüp p. N. Wirbelth. t. 27, f. 1 und 2. - Nicht selten in den abyssinischen Gebirgsgegenden.

243. Pratincola (Koch) albofasciata, Rüpp. Gebirgen.

Rüpp. Syst. Übers. t. 16. - Einzeln auf den abyssinischen

* 244. Pratincola melanoleuca, He ugl.

In Gebüschen der wärmeren Gegenden von Woggara und Simehn in Abyssinien.

245. Pratincola melanura, Rü p p.

T e mm. Pl. col. t. 257, f. 2. - In Arabien, vorzüglich in TamarixGebüschen, sehr selten in Nubien.

* 246. Pratincola Hemprichii, E h renb.

In Abyssinien, Sennaar, und Nubien.

*247. Pratincola caffra, Licht.

In Nubien und Abyssinien.

248. Pratincola Rubetra, Linn.

N a u m. V. D. t. 89. f. 3 und 4. - In ganz N. O. Afrika und Arabien im Winter und Frühjahr.

*249. Pratincola Rubicola, Linn.

N a um. V. D. t. 90, f. 3 und 4. - In ganz N. 0. Afrika. In Abyssinien eine klimatische Varietät (viel intensiver gefärbt, als die europäische) das ganze Jahr hindurch.

250. Thamnobia (?) (Swa in s on) albiscapulata, Rüpp.

R ü p p. N. Wirbelth. t. 26, f. 1. - In Abyssinien nicht selten.

251. Thamnobia (?) semirufa, Rüpp.

R üpp. N. Wirbelth. t. 25, f. 1 und 2. - Häufig in Ost- und CentralAbyssinien.

An m. Saxicola moesta, Licht. und S. gutturalis, Hempr. und Ehren b. oder S. salina, Evers m. sollen in Ägypten und Nubien vorkommen. Ich kenne diese Arten nicht.

\section{Parinae.}

252. Parus (Tıinn.) leucomelas, Rüp p.

Rüp p. N. Wirbelth. t. 37, f. 2. - Paarweise in Sennaar, Fazoglo und Abyssinien, jedoch nicht in den Gebirgsgegenden. 
253. Prerus dorsutus, Rüpl.

R ï p p. Syst. Übers. t. 19. - P. lenconotos, Guer. (?) - Sehr häufig auf den Gebirgen von Waggara und Simehn, auch in Schoa.

23̈4. Purisoma (Swainson) frontule, R üpp.

R ü pp. Syst. Über's. 1. 22. - Einzeln in Ost-Abyssinien und Sehoa.

A n m. In der Sammlung des llerzogs P a u $\mathbf{v}$. Wiir t e m b e r g sah ich wem ich mich noch recht erimnere - einen als Parus niger aufgestellteu Vogel aus Sennaar oder Fazoglo. Ich konnte denselben übrigens nicht genau untersuchen, und es ist möglich, dass dieser Vogel identisch ist mit meinem Melaenormis melus aus Abysinien.

5. Ilotacillinac.

235. Hotacilla alla, Linn.

Buff. Pl. enl.t. 632. f. 2. - N a um.V. D. t. 86, f. 2 - Häufig in ganz N.0. Afrika und Arabien, das ganze Jahr über.

236. Motacilla caperesis, 职ht.

Le Vaill. Ois. d'Afr. t.178. - In Ober-Ägypten und südwärts lïngs des Nils; kommt auch in Abyssinien vor.

23\%. Motrcilla lomgicaudata, Püp p.

R ӥp p. N. Wirb. 1. 29, f. 2. - Nicht selten an Gebirgsbächen in Abyssinien.

2:S. Burlytes (Cuv.) flume, Linn.

Buff. Pl. eul. t. 674. - N a um. V. D. t. 88. - Überwintert südlich von Nubien. In Frïirjalı und Herbst auf dem Durehzuge in Ägypten, wo auch einzelne Pare zu bleiben scheinen.

*259. Budyles bourulus. Linn.

Buff. Pl. eul. 1. 28, f. 1. - Naum. V. U. t. 87. - Nicht häufig im Herlsst und Frïhigallr in $\ddot{A g y p t e n . ~ U ̈ b e r w n t e r t ~ i n ~ A b y s s i n i e n . ~}$

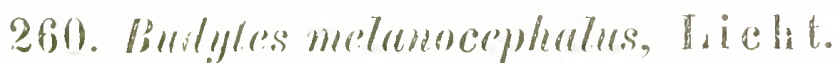

R ïpl). All. t. 33, ligg. b. - In Frälijiahe und flerbst auf dem

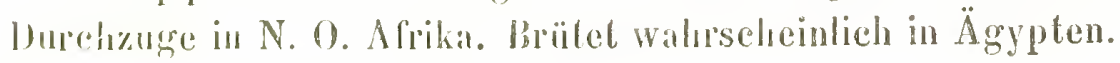

261. Anthus arborens, Brechst.

liuff. P’. enl. t. 660, f. 1. - Naum. V. D. 1.84, f. 2. - In Winter in $\ddot{\Lambda}$ gipten.

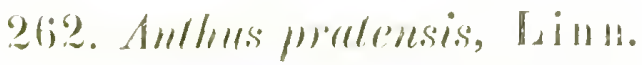

beser. de l'tigypte 1. 13, f. 6. - Naun. V. D. t. 84, f. 2. Wie der vorthergehente.

263. Anlluss cervimus, l'all.

A. Cerilii, Savigny, Deser. de l'ligyt. 1. H. f.6. - A. pratensis, Naum. V. D. t. 8:3, P. 1. (?) - In Frühigalır häufig in Unter-Ägypten. 
264. Anthus campestris, Be chst.

N a u m. V. D. t. 84 , f. 1. - Buff. Pl. enl. t. 661, f. 1. - In ganz N. 0. Afrika und Arabien. Ob Standvogel?

265. Anthus aquaticus, Bechst.

A. spinoletta, Linn. - Na um. V. D.t. 85, f. 2, 3 und 4. - B u ff. Pl. enl. t. 661, f. 2. - Im Winter und Frühjahr in ganz N. 0. Afrika südwärts bis Abyssinien und dem Bahr el abiad beobachtet. Hält sich auch an den Ufern des rothen Meeres auf.

266. Anthus sordidus, Rüpp.

Rüp p. N. Wirb. t. 39, f. 1. - In Abyssinien Standvogel.

267. Anthus cinnamomeus, Rüpp.

In Abyssinien.

\section{B. TURDIDAE.}

1. Turdinae.

268. Bessonornis (Smith.) semirufa, Rüpp.

R ü p. Syst. Übers. t. 21. - Häufig in Abyssinien bis 11,000 F. Höhe.

*269. Bessonornis Monacha, He ugl.

Heugl. Beitr. t. 15. - In Rosseres und Fazoglo.

270. Petrocinla (Vig.) saxatilis, Buff.

Buff. Pl. enl. t. 562. - Naum. V. D. t. 73. - Nicht selten in N. 0. Afrika und Arabien, wie es scheint blos vom September bis April.

271. Petrocossyphus (B o je) cyanus, Buff.

B u ff. Pl. enl. t. 562.-N a u m.V. D. t. 72. Wie die vorhergehende.

272. Turdus Merula, Linn.

$\mathrm{N}$ a u m. V. D. t. 71. - Soll im Winter in Ägypten vorkommen.

273. Turdus musicus, Linn.

B uff. Pl. enl. t. 406. - N a um. V. D. t. 66, f. 2. - Im Winter in Ägypten und Arabien.

*274. Turdus olivaceus, Linn.

Le Vaill. Ois. d'Afr. t. 98. - In Abyssinien und am weissen Fluss.

*275. Turdus icterorhynchus, Herzog Paul v. Würte mb erg.

T. libonyana, A. Smith Zool. Ill. t. 38. (?) - In Kordofan, am weissen und blauen Fluss und in Abyssinien.

276. Turdus viscivorus $\mathrm{Lin}$.

Buff. Pl. enl. t. 489. - Naum. V. D. t. 66, f. 1. - Nach Dr. Rüp pell im Winter in Ägypten.

27\%. Turdus pilaris Linn.

B uff. Pl. enl. t. 490. - Naum. V. D. t. 6\%, f. 2. - Nach Dr. Rü ppell im Winter in Nubien. 
278. Cercotrichas (Boje) erythopterus, Linn.

Buff. Pl. enl. t. 3马ú. - Gemein südlich vom $19^{\circ} \mathrm{N}$. B.

279. Pyenonotus (Kuhl) Arsinoë, Licht.

Hizufig in N. O. Afrika südlich vom $24^{0}$ N. B.

280. Pycnonotus Levaillantii, $\mathbb{T}$ em m.

Le Vaill. t. 107, f. 1. - Im Fayum, in Mittel-Ägypten, im peträischen Årabien und an dem Bahr el abiad.

2. Ti im a i n a e.

281. Crateropus (Swainson) leucocephalus, Rü pp.

Rïp p. Atl. t. 4. - Häufig in kleinen Gesellsehaften im Buschwerk südlich vom $10^{\prime \prime} \mathrm{N}$. B.

282. Crateropus leucopygius, R iupp.

R ü p p. N. Wirbelth. t. 30, f. 1. - In Abyssinien.

*283. Crateropus cinereus, He

An den Ufern des Bahr el abiad südlich vom $6^{0} \mathrm{~N}$. B. (Vielleicht identisch mit dem folgenden, von dem er sich blos dureh geringere Grösse, Mangel des sehwarzen, kahlen Fleckens hinter den Augen und graue Iris unterseheidet.)

284. Crateropus plebejus, Rüpp.

R iipp. Atl. t. 23. - In Kordofan.

2S3. Crateropus rubiginosus, R i i p p.

Rüp p. Syst. Übers. t. 19. - In Schoa.

286. Crateropus rufescens, Heugl.

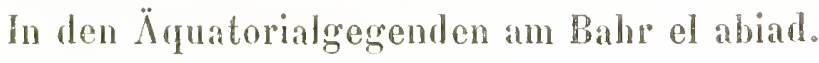

287. Craleropus guttatus, Heugl.

It e ung l. Beitr. t. 16. - Vom $10^{0}$ N. B. südwïrts, häufig am Ufer des Bahr el aliad.

288. Couleropms limbalus, Hartis.

R ii pr. Syst. Ühers. S. 48. - Aus Sehoa.

289. Sphemura Acariae, Li idht.

R ï pl. All. 1. 28. - In Ägyplen, Nubien und Sennaar.

290. Sphermure squamicepses, Rizpp.

R ӥjр. All. 1. 12. - Im pelräischen Arabien.

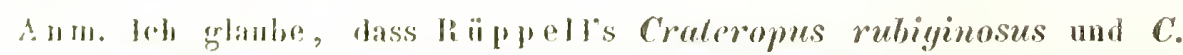
meferens, Mili fher su Sphenura za zihlen sind; doch gehen leide Subgeneru so in cinander über, dass eine Trennung dersethen nicht eimna! aweckmässig ist. 


\section{Oriolinae.}

291. Oriolus Galbula, Linn.

B u f f. Pl. enl. t. 26. - Na um. V. D. t. 61. - Von August bis April in N. 0. Afrika, geht wenigstens bis Kordofan und Galabat südwärts. Männchen im Sommerkleide sind mir aber nie vorgekommen, mit Ausnahme eines aus Ben-Ghasi mir zugeschickten Exemplares.

*292. Oriolus larvatus, Licht.

Am Ufer des Bahr el abiad südlich vom $10^{\circ} \mathrm{N}$. B.

293. Oriolus Meloxita, Buff.

R üpp. N. Wirbelth. t. 12, f. 1. - Häufig in Abyssinien und 0st-Sennaar. Geht nicht über 7000 F. Meereshöhe.

*294. Oriolus chryseos, He ugl.

0. aureus, Le V a ill. Ois. d'Afr. t. 260 (?) - O. auratus. Vi eill.(?) - Swains. Birds of West-Afr. II., t. 1. - H eug l. Beitr. t. 19, fig. 1. - Bei Rosseres am blauen Fluss, in Fazoglo, Galabat und den KollaLändern von West-Abyssinien. Ich glaube ihn auch in eine Sammlung vom Bahr el abiad gesehen zu haben.

\section{c. MUSCICAPIDAR.}

1. Illuscic apinae.

295. Muscicapa Grisola, Linn.

Buff. Pl. enl. t. 565 , f. 1. - $\mathrm{Na} \mathrm{u} \mathrm{m.} \mathrm{V.} \mathrm{D.} \mathrm{t.} \mathrm{64,} \mathrm{f.} \mathrm{1.} \mathrm{-} \mathrm{Den}$ Winter über in ganz N. 0. Afrika.

*296. Muscicapa minuta, Heug l.

Heugl. Beitr. t. 17, f. 2. - Einzeln in buschigen Thälern von Abyssinien.

*297. Muscicapa atricapilla, L in n.

M. luctuosa, S copoli. - Naum. V.D. t. 64, f. 2,3 und 4. Im März und April in Unter-Ägypten.

298. Muscicapa albicollis, T' e m m.

M. colluris, B ech st. - $\mathrm{N}$ a u m. V. D. t. 65 . - B üf f. Pl. enl. t. 365, f. 2. Im März und April häufig in Dattelwaldungen und Buschwerk in Unter-Ägypten und Arabien.

299. Muscicapa semipartita, R ü p p.

R üpp. N. Wirbelth. t. 40. - Einzeln in Abyssinien, sehr häufig an den Ufern des Bahr el abiad, südl. vom $10^{\circ}$ N. B. - Nach Rüppell auch in Kordofan.

300. Muscicapa (?) chocolatina, R ü p p.

R ü p p. Syst. Übers. t. 20. - Häufig im Buschwerk in Abyssinien. Geht bis über 10,000 F. Höhe. 
*301. Muscicapa pallida, Il eug I.

Einzeln in der Kolla und in Kordofan.

302. Muscipete (Cuv.) melenogrester, $\mathrm{S} w$ a in s o n.

Ilïufig in Abyssinien, Sennaar, IKordofan, auf dem Bahr el abiad. Variirt mit weissem oder rostrothem Schwanz, zuweilen sogar ist eine der verlängerten Schwanzfedern von der einen, die anderen von der andern Farbe.

303. Platysteira (Jardine) senegalensis, Linn.

L. Vaill. Ois. d'Afr. t. 161, f. 1 und 2. - Nicht selten in Abyssinien, Sennaar, Kordofan und auf dem weissen Flusse. Die Iris dieses hübschen kleinen Fliegenfüngers ist schön schwefelgelb und seine Stimme gleicht dem reinsten Glockentone.

*304. Bradornis (Smith) variegatus, $\mathrm{Heugl}$.

II e u g 1. Beitr. t.1\%, f. 3. - (Vielleicht identisch mit Bradornis mariquensis, Snith, Zool. Ill. Birds t. 113.) - Blos ein Exemplar vom Berge Lokoja am Pahre el abiad.

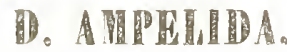

1. Gampepharianar.

303. Conucalis (Cuv.) pectorulis, Jal dine.

Jaldine, Ornith. Illustr. t. $ّ \%$. - In kleinen Gesellschaften in der Kolla West-Abyssiniens, seltener im Osten dieses Landes.

306. Cehlepyris (Cuv.) Ignatii II eugl.

II e u giol. Beitr. t. 18, f. 1. - (Vielleicht das Weibehen vom folgrenden?) Selten am Bahr el abiad, zwischen dem 4--30 N. B.

307. Cehlepyris phönicen, Swainson.

Swainson. liirds of Western Afr. 1. t. 2\% und 28. - Einzeln in Sennaar und Fazoglo, häuliger in Abyssinien, vorzüglich auf Juniperus-Bäumen.

308. Ceblepyris (?) isubellim, He ugl.

Hengl. Prit.r. 1, 18, f. 2. Finzeln in Ost-Abyssinien.

\section{Il) i s, II r i II it e.}

309. Mrelaenmmis (G. R. Gray) edoloides, Swainson.

Sw a inson, !hirds of Western Afr. I. t. 29. - In Schoa. (R ü p p.)

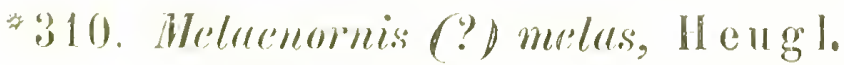

Beitr. t. 17, f. 1. - In Abyssinien und Fazoglo.

"11. Jirmmes (Virill.) lugubris, th then b.

Edolius divaricatus, Auel. - Ehrenb. Symbol. physic. Aves t. 8. - Gremein sïdlich yom 1:0" N. R. 


\section{E. LANIDAE.}

1. I a n in a e.

312. Lanius excubitor, Linn.

Buff. Pl. enl. t. 445. - N a um. V. D. t. 49. - Im Winter in Ägypten, Arabien und Nubien.

*313. Lanius dealbatus, Defil.

Am Bahr el abiad.

*314. Lanius excubitorius, Des Mur es.

Le febvre, Voy. en Abyss. t. 8. - L. princeps, Cabanis. L. macrocercus, D e fil. - In Schoa und am Bahr el abiad südlich vom $10^{0} \mathrm{~N}$. B.

*315. Lanius leuconotus, He ugl.

An der ägyptischen Küste des rothen Meeres angetroffen.

316. Lanius minor, Linn.

B u ff. Pl.enl.t. 32, f. 1. - N a u m. V. D. t. 50. - Im Winter in ganz N. 0. Afrika, wahrseheinlich auch den Sommer über in Abyssinien.

317. Enneoctonus (Boje) rufus, Briss.

B u f. Pl. enl.t. 9, f. 2. - Na u m. V. D. t. 51. - Häufig in ganz N. 0. Afrika.

318. Enneoctonus Collurio, B ris s.

L. spinitorquus, Auct. - Bu ff. Pl. enl.t. 31, f.2. - N a u m. V. D. t. 52. - In ganz N. 0. Afrika.

*319. Enneoctonus frenatus, Lich t.

In Abyssinien und am Bahr el abiad.

*320. Enneoctonus ferrugineus, H e u g I.

He u gl. Beitr. t. 19, f. 2. - L. ruficaudus, Brehm (?) - Wie der Vorige.

*321. Enneoctonus paradoxus, Br e hm.

Im südlichen Nubien. - Ähnlich dem L. ruficeps, aber mit durchgehender weisser Binde an der Schwanzbasis.

322. Nilaus capensis, $\mathrm{S} w$ a in s on.

L. Brubru, La th. - Le Vaill. Ois. 1. 71. - Häufig in Abyssinien, Sennaar und am Bahr el abiad.

323. Telophorus (Swainson) aethiopicus, Vieill.

Rü p p. Syst. Übers. t. 23. - In Abyssinien, Sennaar, am Bahr el abiad und in Kordofan.

324. Telophorus erythropterus, Shaw.

B u f. Pl. enl. t. 479, f. 1. - Südlich vom $15^{0}$ N. B. nicht selten. 
325. Telophorus (?) collaris, Lath.

Jard. IIl. t. 52. - In Abyssinien und am Bahr el abiad.

326. Prionops (Vi eill.) eristutus, Rü p p.

Rüp p. N. Wirb. t. 12,f. 1. - Pr. poliocephalus, Salt. Trav. App. p. $00(?)$ - Südlich rom $1 \breve{g}^{0}$ N. B. in kleinen Truppen. Ob Pr.talacoma rorkömmt, kann ich nicht geradezu behaupten, doch habe ich Exemplare am Bahr el ahiad eingesammelt, die auf die Smith'sche Abbildung und Beschreibung ziemlich passen.

327. Eurocephalus (A. Sinith) Rüppellii, B on ap.

R üp p. Syst. Übers. t. 27 als E. anguitimens, S mith. - In Schoa und am Balre el abiad, südlich vom $8^{0} \mathrm{~N}$. B.

*328. Convinella (Less.) affinis, Heugl.

H e ug I. Beitr. t. 19, fig. 3. - Häufig am Bahr el abiad südlich vom go N. B.

2. Tham nophilinae.

329. Dryoscopus (Boje) cublu, Lath.

L e V a ill. Ois. t. 73. - Südlich vom $15^{0} \mathrm{~N}$. B. häufig.

330. Luniruins (Vieill.) cruentatus, whenb.

Ehrenb. Symb. phys. Aves t. 3. - An der abyssinischen Iíüste und in Fazoglo ziemlich läulig. - Geht nordwärts bis in die Länder der Boklıos und Sauakin.

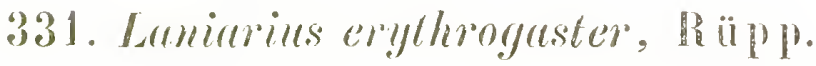

Rë̈pp. Atl. L. 29. - Malaconotus roseus, I a d. und Selb. Süjlich vou $1: 3^{\circ}$ N. B. in den Nil-Län?ern. In Ost-Abyssinien nicht von mir beobachtet.

932. Malaconotus (Swainson) Irerus, Vieill.

H. alivacens, Vieill. Gal. t. 129. - M. poliocephalus, Swa inson (?) - In Kordofan, an weissen und blauen Fluss, in der Kolla von W.-Abyssinien einzehn aul Ilochbäumen.

393. Haluconolus similis, smith.

H. wryssogrester, Swains on. - Sm ith, IHI. Zool., Birds t. 46. Rïpp. Syst. Übers. 1. 24. - Einzeln in Fazoglo, selten an Mareb und in Sirhoa.

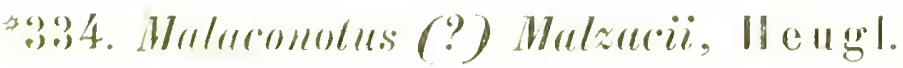

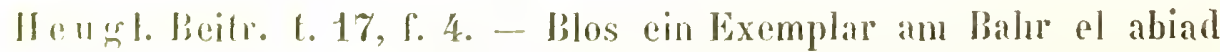
bri den liohrr-Nergern aufgefunden. -.. (Diese Art gehört vielleieht zum Genus Trichophorus.) 


\section{Conirostres.}

\section{A. CORVIDAE.}

1. Callaeatinae.

335. Ptilostomus ( $\mathrm{S}$ wa in $\mathrm{s} \mathrm{n}$ ) senegalensis, Gmel.

Buff. Pl. enl. t. э38. - Einzeln und in kleinen Gesellschaften in Abyssinien, Kordofan und häufig auf dem weissen Fluss.

*336. Ptilostomus poëcilorhynchus, Wagl.

Auf dem Bahr el abiad.

\section{Corvina e.}

Die Raben im Allgemeinen heissen auf arabisch „Ghurab“ (غراب). 337. Pica (Briss.) caudata, Ray.

Corvus Pica, Linn. - B uff. Pl. enl. t. 338. - N a um. V. D. t. 56, f. 2. - Nach R üppell im Winter in Unter-Ägypten.

338. Corvus Cornix, Linn.

Var. Na um. V. Deutschl. t. כ̈4. - Häufig in Unter-Ägypten und Arabien, das ganze Jahr hindurch. Brütet im Frühjahr auf Sykomoren, Palmen und Acacia Lebek. Ob Corv. Corone wirklich vorkommt, kann ich nicht angeben.

339. Corvus Monedula, Linn.

Buff. Pl. enl. t. 523. - Naum. V. D. t. 56, f. 1. - Nach Dr. R ü p pell häufig in Unter-Ägypten. Von mir nie beobachtet.

340. Corvus frugilegus, Linn.

Buff. Pl. enl. t. 484. - Naum. V. D. t. 55, f. 1 und 2. - In Unter-Ägypten. Scheint auch den Sommer über zu bleiben.

*341. Corvus minor, H eugl.

In der Wüste bei Suez, im peträischen Arabien.

342. Corvus umbrinus, Has selq.

In den Wüsten von ganz N. 0 . Afrika bis zum $13^{0}$ N. B. beobachtet. Heisst auf arabisch "Ghuráh nochi" (غراب زوحى).

343. Corvus affinis, Rüpp.

R üp p. N. Wirbelth. t. 10, f.2. - Sehr gemein in Abyssinien, bis zu $11.000 \mathrm{~F}$. hoch, oft in ungeheuren Gesellschaften. Einzeln in Kordofan.

344. Corvus capensis, Le Vaill.

Le Vaill. Ois. d'Afr. t. 52. - Einzelner als der vorhergehende. In Kordofan, Sennaar und Abyssinien, wo ich ihn auf den Gebirgen von Simehn noch antraf.

345. Corvus scapulatus, Da ud.

C. leuconotos, S w a in s o n, Birds of Western Afr. I. t. 5. - Sehr gemein südlich vom $20^{\circ} \mathrm{N}$. B. 
346. Corvultur (Is ess.) crossirostris, Rïipp.

R ̈̈pp. N. Wirbelth. t. 8. - Gemein in Abyssinien, namentlieh in den Gebirgsgegenden, von wo aus er sieh aber bis Galabat und Taka verfliegt. Ausser seinem dem des C. Corax ähnlichen Ruf, lïsst er zuweilen im Fluge ein ganz sonderbares langgezogenes pre-è-ë-ë-è-ë-ë hören. Ich fand sein Nest blos ein einziges Mal an einem ganz unzugriinglichen Orte über einem hohen Wasserfall auf Lianen in Monate Februar.

\section{Frogilinar.}

34\%. Fregilus (Cuv.) graculus, Linn.

Buff. Pl. enl. 1. 23:3. - N a u m. V. D. t. 3\%, f. 2. - Auf den Hochgebirgen des peträisehen Arabien und Abyssiniens; in Simehn bis zu 14.000 F. Höhe. (Nicht von mir selbst beobachtet.)

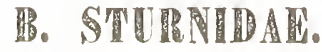

1. Ptilonorlaye hinate.

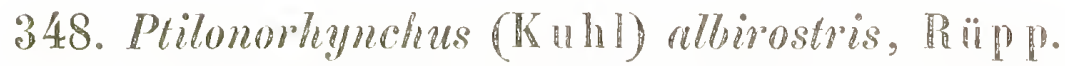

R üp p. N. Wirbelth. t. 9. - Hïufig in Ost- und Central-Abyssinien, in grösseren Gesellsehaften auf Buschwerk und in alten Gebïuden.

349. Lamprotornis lencoguster, Te mm.

Bu f f. Pl. enl. t. 648, 1. -- In kleinen Gesellsehaften in den wïrmeren Gegenden Abyssiniens, vorzüglieh längs der Ufer fliessender Gewässer.

330. Hamprotormis nitens, "l" emm.

P.uff. Pl. enl. t. 861 . - Sehr gemein südlich vom $20^{\circ}$ N. B.

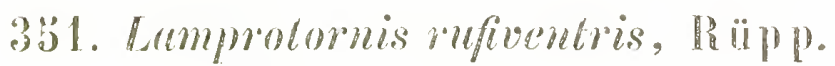

R ï p p. N. Wirbelth. 1. 11, f. 1. - Überall hïufig südlich vom $18^{0} \mathrm{~N} . \mathrm{P}$.

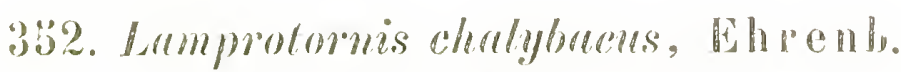

Ehrenb. Symb. Plyys. Aves. I. t. 10. - In Abyssinien.

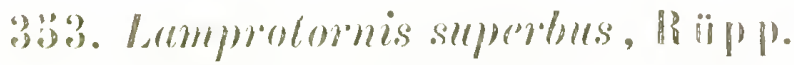

Rii p p. Syst. Uloers. 1.26. - An Bahr el abiad gemein, aber nicht mördlicher als $8^{\prime \prime}$ N. B. - In Sehoa.

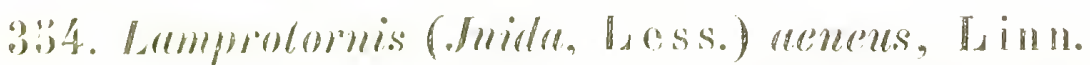

Le Vaill. Ois. d'Afr.t. S\%. - In Kordolan, Sennaar und Abyssinien, vorräglich in der Wald-Region.

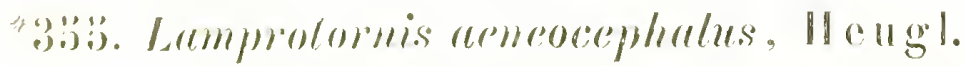

In Mbyssinien, Kordofan, Semnar und auf dem Bahre el abiad.

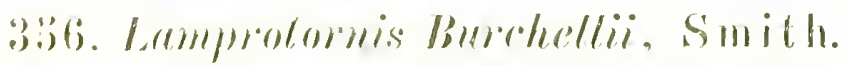

S smill, Ill. Zool. of South Afr. Birds. t. 4\% - Lamprotomis purpuroptere, Riipl. Syst. Übors. 1. 2:3. - Eimzeln is (Kordofan?) llessinien. - In Sehoa. 
357. Lamprotornis morio, Te m m.

Le Vaill. Ois. d'Afr. t. 83. - Gemein in Abyssinien, einzeln in Fazoglo und Kordofan. Im Spätherbst 1851 auf Palmen und Tamarix mannifera in Wadi-ferân im peträischen Arabien beobachtet.

358. Lamprotornis tenuirostris, $\mathbf{R}$ ü p p.

R ü p p. N. Wirbelth. t. 10, f. 1. - In Abyssinien ziemlich häufig.

\section{Buph a gin a e.}

359. Buphaga (Linn.) erythrorhyncha, Stanley.

Tem. Pl. col. t. 465. - B. abyssinica, Eh renb. Symb.t. IX (?) In Abyssinien, Fazoglo und am weissen Fluss.

*360. Buphaga africana, Linn.

Häufig in Galabat, West-Abyssinien, im Marebthal und auf dem Bahr el abiad. - Nie in Gesellschaft mit der vorigen Art.

3. Starninae.

361. Dilophus (Vi eill.) carurculatus, Linn.

Le V aill. Ois. d'Afr. t. 93. - In Abyssinien das ganze Jahr hindurch, an dem weissen und blauen Fluss, nach R üp pell auch in Nubien.

362. Sturnus vulgaris, Lin $\mathrm{n}$.

B uff. Pl. enl. t. 75. - Na um. V. D. t. 62. - Im Winter in ganz Ägypten und im peträischen Arabien. - St. unicolor, T e mm. ist mir dagegen nie vorgekommen.

\section{BRINGILLIDAE.}

\section{P I o c in a e.}

Alle Finken heissen auf arabisch "Asfur" (عصنور).

363. Textor Alecto, $\mathrm{T}$ e $\mathrm{mm}$.

Te mm. Pl. col. t. 446. - In Kordofan, Sennaar, Fazoglo und Abyssinien.

364. Textor Dinemellii, Horsfield.

G. R.G ray, Genera of Birds I. t. 87. fig. dext. - R ü pp. Syst. Übers. t. 30. - In Schoa und auf dem Bahr el abiad südlich vom $8^{0} \mathrm{~N}$. B. in grossen Schaaren.

365. Ploceus (Cuv.) flavo-viridis, R üp p.

R üp p. Syst. Übers. t. 29. - Häufig in grossen Flügen. In Abyssinien, Schoa und Fazoglo.

*366. Ploceus affinis, Heugl.

Am Bahr el abiad.

367. Ploceus Galbula, Rüpp.

Rüpp. N. Wirbelth. t. 32, f. 2. - Häufig an der abyssinischen 0stküste, namentlich im Marebthal. 
36S. Ploceus larvatus, $\mathbb{R}$ ii pp.

R ü p. N. Wirbelth. t. 32, f. 1. - Haifig in Abyssinien und in len Vorgehirgen der Nil-Quellen-Länder (zwisehen dem 4-60 N. B.).

*369. Ploceus aurantius, Vieill.

Nieht seiten in Tigréh um Wohnungen, in deren Nähe er sein Nest auf Bäumen aufhängt.

*370. Ploceus auramlitepes, II ougl.

If e ugl Beitr. t. 20, f. 2, und t. 41, das Ei. - Plos in der Nïhe von Chartum in Gärten und am Bahr el abiad beobachtet.

*3r1. Ploceus citreomeha, Il eugl.

Im südliehen Fazoglo.

37\%. Plocens intermedius, Ir a r is.

Besehr. in R üp p e ll's Übers. S. 71. - N e ugl. Beitr. t.20, fig. 1. - In Schoa und bei den Bari-Negern (zwischen dem 4-马o N.B.) am Bahr el abiad.

3\%3. Ploceus rubiginosus, Rü p p.

IR ï p. N. Wirbelth. t. 32, f. 1. - Von Dr. R üppell in der Provinz Tembehn in Ost-Abyssinien gefunden.

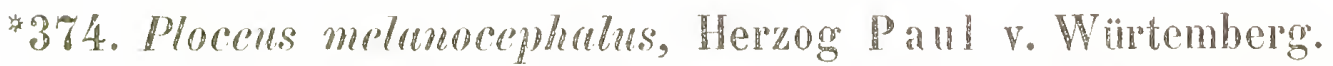

Von Herzog Panl v. Würtemberg im südlichen Nubien entdecht.

* ॐró. Ploceus erythrophthalmos, II e ugl.

Einzeln in Ost-Sennar.

376 . Placens erythrocephralus, Ha lo is.

Rïp p. Syst. Übers. S. \%1. - In Schoa, dem südliehen Sennaar und Fazoglo.

$37 \%$. Ploreus auriforons, T'emm.

T' e 111 m. Pl. col. t.17\%.- Nach Dr. R ü p p ell in Sennaar und Abyssinien.

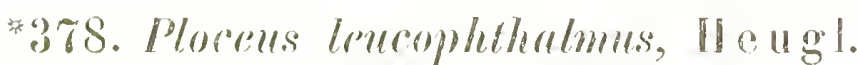

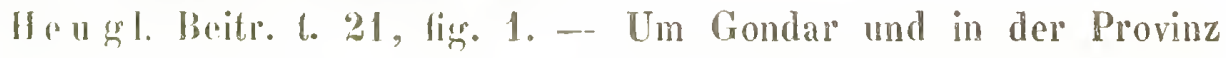
Simeln in Abyssinien.

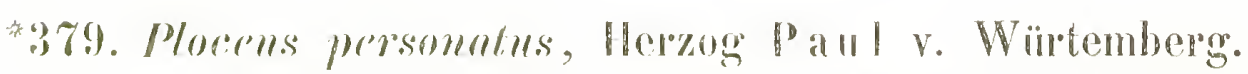

Von Her\%og P’aul v. Würtemberg in Kamamil (südöstl. von Fazoglo) eingresinmolt.

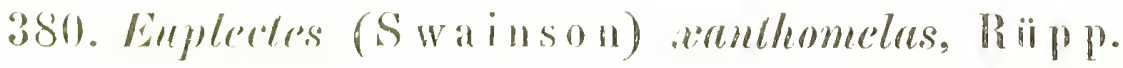

R ï 1). Syst. Übers. 1. 28. - In grossen Flügen in den abyssinischen Gehirgs-tändern ïstlich vom Tana-See.

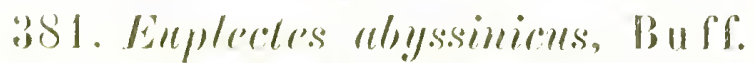

L. Trein, Su ill, Sonth Afr. t. 7 (?) - Wie der vorhergehende.

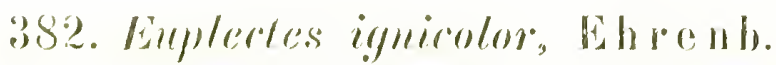

Ehrent. Symb. Phys. Aves. Ł. 2.- In Nubien süallich voun 210 N. B., in Sennaar und Abyssinien. 
383. Euplectes craspedopterus, S chif.

Eine dem E. flammiceps, S w a i n s. ähnliche Àrt, die R üpp el I früher für identisch mit ihr hielt und aufführte; findet sich in kleinen Flügen in ganz Abyssinien.

*384. Euplectes pyrrhocephalus, $\mathrm{Heugl}$.

Heugl. Beitr. t. 21, fig. 3, und t. 22, f. 2. -- Auf dem Bahr el abiad zwischen dem $4-\mathbf{5}^{0} \mathrm{~N}$. B.

*385. Euplectes strictos, Heugl.

Auf den Gebirgen von Simehn in Abyssinien.

*386. Euplectes (?) griseus, Heugl.

Heugl. Beitr. t. 21, fig. 2. - Am Mareb und im südöstlichen Kordofan.

Anmerkung. Ich glaube auch öfter $E$. Ory $x$ beobachtet zu haben.

2. cocethraustinae.

*387. Coccothraustes (?) (B riss.) sanguinirostris (?) Lin n.

Euplectes Quelea, Buff. Pl. enl. t. 223 und 183 (?) - E. gregarius, Herz. P a ul von Würtemberg. - In zahlreichen Flügen in Fazoglo, Sennaar und 0st-Abyssinien. Erscheint mit den Sommerregen auf einige Monate in Chartum.

*388. Coccothraustes (?) scutatus, He ugl.

Vielleicht Fringilla cucullata, Vieill. - Heuglin Beitr. t. 23, fig. 3. - Blos ein Exemplar in den Kolla-Ländern von West-Abyssinien beobachtet.

389. Coccothraustes (?) cantans, Linn.

Brown, Illust. t. 2\%, f. 2. - In Nubien, Kordofan und Sennaar, oft in zahlreichen Flügen.

390. Vidua (Cu v.) paradisea, Linn.

Häufig südlich vom $15^{\circ}$. N. B.

391. Vidua erythrorhyncha, Swainson.

Vieill. t. 36. - Wie die Vorhergehende.

392. Coliuspasser (Rüpp.) macrurus, Linn.

Brown, Illust. t. 11. - Buffon t. 183. - Schaarenweise auf Arundo Donax und Buschwerk in den Umgebungen von Gondar und in Tigréh.

393. Coliuspasser torquatus, $\mathrm{R}$ ü p p.

R üp p. N. Wirbelth. t. 36, f. 2. - Im östlichen Abyssinien.

*394. Coliuspasser phöniceus, $\mathrm{He} \mathrm{u} \mathrm{gl.}$

Heuglin Beitr. t. 20, fig. 3 a und b. - Bis jetzt blos am SobatFlusse gefunden. 
3. Fring illinae.

393. Fringilla (Estrelda, Swa ins on) coerulescens, Linn.

Vieill. Ois. chant. 1. 1\%. - Südwärts rom $18^{0} \mathrm{~N}, \mathrm{~B}$.

396. Fringilla (Estrelda) bengalus, Lin $\mathrm{n}$.

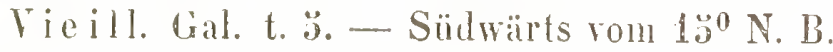

397. Fringilla (Estrelda) minima, Vi eill.

Vi e ill. Taf. 10. - Sehr häufig sü̈lich rom $22^{0} \mathrm{~N}$. B. in Wohnungen und in Waildern.

398. Fingilla (Estrelda) cinerea, Tieill.

Vi eill. Taf. 6. - Südlich rom $1 \frac{4}{4} \mathrm{~N}$. B.

*399. Fringillu (Estrelda) flavirentris, He ug $\mathrm{I}$.

Fr. melpoda, Vieill. (?) - Blos im üstlichen Abyssinien eingesammelt und bei Gondar bemerkt.

400. Fringilla (Pyteliu, Swains on) elegans, Vieill.

Vi e ill. T. 2o. - In Nubien, Sennaar, Kordofan und Abyssinien.

401. Fringilla (Pytelin) lineatr, He $u g 1$.

He ugl. Beitr. t. 23, f. 2. -- Fr, phönicoptera, $\mathrm{S} w$ a ins. Birds of W. Afr. I. t. 16 (?) - In den Kolla-Lündern von West-Abyssinien.

*402. Fringilla (Amadina, Swainson) squamifrons, A. Smith.

Sinith, Ill. of South Afr. t. 96. - In den Kolla-Ländern von West-Abyssinien und in Beni-Schangollo.

403. Fringilla (Amadina) detruncata, Lie ht.

Vieill. 1. 38. - Amadina fasciata. Swainson, Birds of W. Afr. t. 1:. - Südlich vom $14^{\circ}$ N. B.

404. Fringilla (Amalina) nitens, Vieill.

Vieill. 1. 21. - Gemein in Nubien, Kordofan, Sennaar und 1byssinien.

403 . Fringilla (Amadina) frontalis, Vieill.

Yieill. t. 16. - Ehendaher.

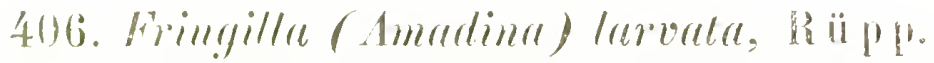

R ïpp. N. Wirbelth. t. 36, t. 1. - Häufig in den Kolla-Ländern von West-1byssinien lis Gatabat, seltener im Takasseh-Quellenland.

407. Fringilla (Amalina) polyzona, Temm.

Temm. P. col. 1. 221, f. 2 und 3. -.- In Abyssinien.

"qus. Frimgilla (Dryospran, Keys. et Blas.) Serinus, Linn.

Nit un. Y. L. t. 123. - In Hiirz nicht selten in Unter-Ägypten.

"409. Fringilla ( Dryospira) leucomygin, Heugl.

Hangl. Beitr. 1. 24, f. 3 und 4. - In Ost-Sennar und Galabat, vielleirlat anclu in Kordofint. 
410. Fringilla (Dryospiza) xanthopygia, R üpp.

Rüpp. N. Wirbelth. t. 35, fig. 1. - In Abyssinien, vorzüglich im Takasseh-Quellenland.

411. Fringilla (Dryospiza) tristriata, R ü p p.

Rüpp. N. Wirbelth. t. 35, f. 2. - Häufig in Central- und OstAbyssinien. Geht über $10,000 \mathrm{~F}$. hoch

*412. Fringilla (Dryospiza) aurifrons, Heug $\mathrm{l}$.

He ug l. Beitr. t. 23, fig. 1. - Crithagra chrysopyga, S wains. Birds of West. Afr. t. 17 (?) - In 0st-Sennaar.

413. Fringilla (Dryospiza) nigriceps, R ü p p.

Rüpp. N. Wirbelth. t. 34, fig. 2. - In Abyssinien in grossen Flügen. Bis $11,000_{a} \mathrm{r}$. Meereshöhe beobachtet.

414. Fringilla (Dryospiza) citrinelloides, Rüpp.

R ü p. N. Wirbelth. t. 3:, f. 1. - Ebendaher.

415. Fringilla (Dryospiza) lutea, T e mm.

Temm. Pl. col. t. 365. - Nicht selten in Nubien, Sennaar und Kordofan. In Chartum erscheint sie in grossen Flügen zu Anfang der Sommerregen und zieht im September wieder weg.

416. Fringilla (Acanthus, Keys. et Blas.) Carduelis, L in n. Im Winter einzeln in Ägypten.

417. Fringilla (Acanthus) Linaria, Linn.

B uff. Pl. enl. t. מ1, f. 1. - Nach R ü p pell im Winter in Ägypten.

*418. Fringilla (Acanthus) chrysomelas, H e ugl.

Heugl. Beitr. t. 24, f. 5 und 6. - In Kordofan und Sennaar.

419. Fringilla (Linota, B o n a p.) cannabina, Lin n.

$\mathrm{N}$ a u m. V. D. t. 126. - - Im Winter in Ägypten.

*420. Fringilla (Pyrgita, C uv.) coelebs, Linn.

Nau m. V. D. t. 118. - Im Winter in Ägypten.

*21. Fringilla (Pyrgita) molybdocephala, He ugl.

H e u gl. Beitr. t. 20, fig. 4. - In Flügen am Bahr el abiad zwischen dem $7-9^{0} \mathrm{~N}$. B., vorzüglich im Innern des Landes an den Flüssen Faff, Namm und Niebor.

422. Fringilla (Passer, Ray) domestica, Linn.

423. Fringilla (Passer) cisalpina, T emm.

Nach Rüppell häufig in Ägypten und Nubien.

424. Fringilla (Passer) hispaniolensis, T e mm.

Deser. de l'Egypte.t. 3, fig 7.- Schaarenweise in Unter-Ägypten. (Rüp pell.) 
*423. Fingilla (Passer) simplex, Licht.

Blos in der Bajuda-Wüste, in Kordofan und Sennaar.

*426. Fringilla (Passer) motitensis, A. Smith.

S m ith, Ill. of South Afr. Zool. t. 114.

*427. Frimgilla (Passer) lumata, He ugl.

Heunl. Beitr. t. 24, f. 1 und 2. - In Ost-Sennaar.

428. Fringilla (Passer) Suainsomii, R ï p p.

R üp P. N. Wirbelth. t. 33, f. 2. - Häufig südlich rom $13^{0} \mathrm{~N}$. B.

4ํ. Fringilla (Passer) montana. Isinn.

B uff. Pl. enl. t. 26\%, f. 1. - In Ägypten.

Anm. Tach Bnnaparte kommen folgende eigentliche Sperlinge in j. O. Afrika vor.

Passer rufipectus, B o nap. Ägrpten.

Passer arboreus, Li cht. Sennaar.

Pusser Rüppellii, B o nap. Ost-Afr.

\section{E.mbrizilla .}

430. Emberisa hortulana. Is inn.

Buff. Pl. enl. t. 247.f.1.- N a um. Y. U.t. 103. - Ich weiss nicht, ob lie Fett-Amer sieh das ganze Jahr in Abyssinien aufhäl oder nicht, glaube aber, dass sie dort Standrogel ist. Fom Jänner his April traf ich sie ïberall ungemein häufig, bis über $10,000 \mathrm{~F}$. Meereshöhe. - Im IIärz $1 S$ ö0 traf ich sie üfters auf Brachfeldern und Zedern bei Alexandria cremeinschaftlich mit $E$. caesia, mit der sie auch in Benehmen und Lockton ganz übereinstimmt.

生31. Emmerisa calesile, R ü ll ll.

R ï p p. Atl. t. 10, fig. 6. - Häufig in Ägฐpten, wo sie brütet, im Winter allch in Abysinien.

432. Emberisa parigasera, R ï ple.

li ii p r. Atl. t. 驾. - In Ost-Sennar und Kordofan, einzeln auf bïmmen und in Wïldern.

439. Emberisa miliaria, Linn.

Buff. Pl. enl. t. 233. - Kaum. V. D. 1. 101, f. 1. -- Im Winter bïufig in lonter-äg!pten.

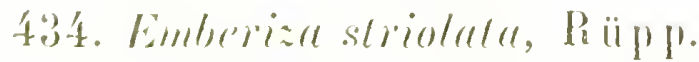

Rüpl. 1tl.t. 10, fo a. - Nach R ï p pell in Nubien. Ich fand sie hlos in Cot-Sennatr und den Hochländern am Dender und Rahad.

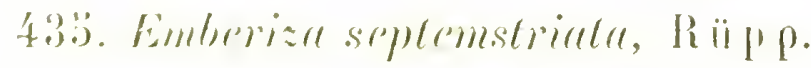

S ï 1'1\% Nene. Wirbelth. t. 30, f. 2. - Von mir blos bei Gondar in fast trockenen Flussbetten und an deren Ufurn beobachtet. Ich fand dort im Jïnner öfters ihr Nest. 
Anm. Wenn ich nicht irre, habe ich in Ägypten auch Emberiza Schöniclus und citrinella bemerkt, doch finde ich keine Notizen mehr hierüber. Jedenfalls werden sie blos im Winter dort vorkommen. $\boldsymbol{E}$. melanocephala und $\boldsymbol{E}$. pyrrhuloides habe ich vergebens gesucht.

436. Plocepasser (A. Smith) superciliosus, Rüpp.

Rüp p. Atl. t. 15. - Häufig in Sennaar und Abyssinien.

437. Plocepasser Mahali, A. Smith.

P. melanorhynchus, Rü p p. - H e ugl. Beitr. t. 22, f. 1. - S mith, IIl. of South Afr. Zool. Birds. t. 63. - In Schoa und am Bahr el abiad südlich vom $10^{\circ} \mathrm{N}$. B.

\section{A l a u din ae.}

438. Certhialauda (S w a in s o n) desertorum, S ta nl ey.

Rüpp. Atl. t. 5. - In Ägypten, Nubien und Arabien. Standvogel.

439. Melanocorypha (Boje) Calandra, L i n n.

B uf f. Pl. enl. t. 363, f. 2. - Naum. V. D. t. 98 , f. 1. - Nach Rüppell häufig in Nubien und Ägypten. Ich traf sie einmal im März bei Alexandria und im Winter in kleinen Gesellschaften mit Alauda ruficeps am Tana-See.

440. Melanocorypha brachydactyla, L e is I.

Leisl. Wetterauer Annalen, VIII, t. 19. - Na u m. V. D. t. 98, f.2. - Im Winter und Frühjahr in Nubien, Ägypten und Arabien.

441. Melanocorypha isabellina, Te m m.

T e m m. Pl.col. t. 244. - In Ägypten, Nubien und Arabien. Zugvogel.

442. Otocornis (B o je) bilopha, $\mathrm{R} \ddot{\mathrm{u}} \mathrm{p} \mathrm{p}$.

Temm. Pl. col. t. 244. - Ist wohl nur Varietät von Alanda alpestris; ich fand sie blos im peträischen Arabien im Sommer. Im Winter ist sie mir dort nicht vorgekommen.

443. Galerida (B o j e) cristata, Linn.

Buff. Pl. enl. t. 503, f. 1. - N a u m. V. D. t. 99, f. 1. - Hänfig als Standrogel in ganz N. O. Afrika.

444. Alauda arvensis, Linn.

B uff. PI. enl. t. 363, f. 1. - Na u m. V. D. t. 100, f. 1. - Im Winter in Nord-Afrika.

*445. Alauda arborea, Linn.

Na u m. V. D. t. 100, f. 2. - Von Dr. A. B rehm bei Alexandria im Winter erlegt.

*446. Alauda praestigiatrix, L e Vaill.

Le Vaill. Ois. d'Afr. t. 190. - (A. marginipennis, P. v. Wü rt e mberg.) - Selten in Sennaar und Kordofan. 
447. Megalophonus (G. R. Gray) ruficeps, Rü p p.

Rüp p. N. Wirbelth. t. 38, f. 1. - Häufig in Abyssinien und OstSennaar. - Geht bis 10,000 F. Meereshöhe.

448. Macronyx ( $\mathrm{S}$ wa in s o $\mathrm{n}$ ) flavicollis, $\mathrm{R}$ ü p p.

Rüp p. N. Wirbelth. t. 38 , f. 2. - Nach Rüppell häufig in Abyssinien. Ich fand ihn blos auf sumpfigen Wiesen in der Provinz Woggara im Februar und März beiläufig auf einer Höhe von 7-9000 F.

449. Pyrrhalauda (S mith) crucigera, T e m m.

Temm. Pl. col. t. 269, f. 1. - In Arabien und Kordofan.

450. Pyrrhalauda leucotis, Sta nle y.

Te mm. Pl. col. t. 269, f. 2. - Sehr häufig in Nubien, Sennaar und Kordofan. Wandert im Herbst (September) öfters in Flügen wie die Lerchen.

A n m. Obwohl die beiden oben angeführten Pyrrhalauden ìm Allgemeinen den Beschreibungen und Abbildungen Temminck's etc. gleichen, so glaube ich doch kaum an die Identität der zwei sudanischen Arten mit der wahren $\boldsymbol{P}$. crucigera und $\boldsymbol{P}$. leucotis. Eine Vergleichung mit Gap'schen Original-Exemplaren kann ich nicht anstellen, muss daher die Entscheidung dieser Frage für die Zukunft verschieben.

\section{Pyr Lulinae.}

451. Pyrrhula (Bris s.) (Erythrospiza, B on.) githaginea, L i ch t.

Te m m. Pl. col. t. 400. - In Mittel- und Ober-Ägypten und Nubien auf Brachfeldern und Felsen. Brütet im April; ob sie Standvogel ist, kann ich nicht genau angeben. Ich fand sie im Febrnar, Mai und Juni, nicht aber im October und November, bei Assuan.

4ว้2. Pyrrhula sinoica, Li c h t.

T e m in. Pl. col. t. 375. - Zum Subgenus Carpodacus, K a u p gehörig. - In peträischen Arabien in kleinen Flügen.

453. Pyrrhula (?) striolata, R ü p p.

R ü p p. N. Wirbelth. t. 37, f. 1. - Zu Scrinus (Boje) nach B o n aparte geliörig. - In Central- und 0st-Abyssinien; geht über 10,000 Fuss Höhc.

\section{COLIDAE.}

454. Colins senegalensis, L in n.

Vieill. Gal. t. 31. - In kleinen Gesellschaften in Nubien, Sennaar, Abyssinien und Kordofan.

45\%. Colius leucotis, R ü p p.

R ïp p. Mus. Senkenb. Vol. III, t. 2, f. 1. - In ganz Abyssinien, im südlichen Sennaar und Fazoglo und an den Ufern des weissen Flusses rom $10^{\circ} \mathrm{N}$. B. südwälls. 


\section{E. BUCEROTIDAE.}

456. Tragopan (Möh ring) abyssinicus, Gmel.

B u ff. Pl. enl. t. 779. - Paarweise in Abyssinien, seltener in Sennaar, Kordofan und längs des Bahr el abiad. Heisst auf arabisch „Abu qarn" (إو قرن), auf amharisch „Abba-Gamba“.

457. Buceros (Linn.) cristatus, Rüpp.

Rü p p. N. Wirbelth. Vögel. t. 1. - In den abyssinischen Tiefländern, namentlich in Godjam, Damot, am Tana-See, häufiger in Schoa.

458. Toccus (Less.) erythrorhynchus, Lath.

B u f. Pl. enl. t. 260, - Gemein südlich vom $16^{\circ} \mathrm{N}$. B.

*459. Toccus nusutus, Lath.

B uff. Pl. enl. t. 890. - Wie der Vorige, doch einzelner.

460. Toccus limbatus, Rüp p.

R üpp. N. Wirbelth. t. 2, f. 2. - In Abyssinien und Kordofan, auch am Bahr el abiad beobachtet.

461. Toccus flavirostris, Lath.

R ü p p. N. Wirbelth. t. 2, f. 1. - In Abyssinien.

*462. Toccus poëcilorhynchus, Vieill. (?)

H e u gl. Beitr. t. 2.. - Bei Chartum und längs des ganzen Bahr el abiad.

\section{F. NIUSOPHAGIDAE.}

463. Turacus (Cuv.) leucotis, Rüpp.

Corythaix leucotis, R üpp. N. Wirbelth. t. 3. - Häufig an baumreichen Wildbächen in Abyssinien; geht nicht über 7-8000 Fuss Meereshöhe.

*464. Turacus leucolophus, Heugl.

Corythaix leucolopha, Heugl. Beitr. t. 26. - Bis jetzt blos am (Berge) Belinia zwischen dem 4. und $5^{0}$ N. B. im Gebiete der BariNeger beobachtet.

465. Chizaerhis (Wagl.) zonura, Rüpp.

Rüpp. N. Wirbelth. t. 4. - Häufig in Abyssinien und am Bahr el abiad, seltener am blauen Fluss, auf Hochbäumen.

466. Chizaerhis personata, Rü p p.

Zool. Transact. V. 3, t. 16. - In Schoa.

467. Chizaerhis leucogaster, Rüpp.

Zool. Transact. V. 3, t. 17. - In Schoa. 


\section{ORDNUNG. SCANSORES (KLETTERVÖGEL).}

\section{A. PSITTACHDAE。}

Auf arabisch heissen die Papageien „Durra“ und,Babagán“(نliz,, dy)). 468. Piomes (Wagl.) Meyeri, Rüpp.

Rüpp. Atlas, t. 11. - In Gesellschaften und einzeln, vorzüglich in der Waldregion, nie nördlich rom $14^{\circ} \mathrm{N}$. B. beobachtet.

469. Pionus flavifrons, Rü p p.

Rüpp. Syst. Übers. t. 31. - In Schoa; wahrscheinlich auch auf dem Pahr el abial in südlicheren Breiten.

470. Pionus mfiventris, Rüpp.

Rüpp. Syst. Übers. t. 32. - In Schoa.

471. Pionus Levaillantii, Kuhl (?)

Le Vaill. Ois. t. 130. - Von Dr. Rüppell in den Gebirgen von Simehn beobachtet. - Ich habe mich in Abjssinien oft nach diesem Vogel erkundigt, konnte aber auch nichts Näheres über ihn erfahren, als dass ein Individum nach Frankreich gesehickt worden sei.

472. Psitucula (Briss.) Trantue, Stanley.

L e ar, Psittac. t. 39. - Häufig in ganz Abyssinien. Ich traf ihn noch bis gegen 9000 Fuss hoch an. Er lebt vorzüglich auf Juniperus-Bäumen.

473. Palneornis (Vigors) cubiculuris, Has selq.

Le Vaill. Perr. t. 22. - Mïufig und oft in grossen Gesellschaften südlich rom $10^{0} \mathrm{~N}$. B. - Soll zuweilen weit nördlicher ziehen und selbst in $\ddot{A} g y p^{t e n}$ vorkommen (?)

\section{IB. PICIDAN.}

1. Buccon inac.

474. Laimodon (G. R. Griay) melanocephalus, R ï p p.

pogonias melenocephalus Rü̈p p. Atl. t. 28. - İn Sennaar und Kordofan (R ïpp.). Ist mir bis jetzt nie vorgekommen.

4rö. Laimodon Vieilloti, Iseach.

L cea c h, Zoblog. Miscell. Vol. II, t.97. - Überall südlich vom $14^{0}$ N.P.

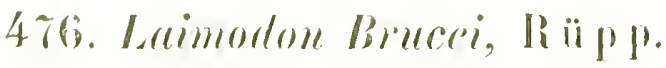

Rï̈p l. N. Wirbelth. 1.20, l. 1.-In $\Lambda$ byssinien, Sennaar und Kordofan.

47\%. Laimodon undulus, Rïpp.

R ӥрр. N. Wirhelth. t. 20, f. 2. - In Abyssinien und Sennaar.

468. Lamordon lencorephahes, II eusl.

H engl. lieitr.1.27, f. 1. - Am weissen Fluss, südlieh vom 80 N.B ziemlich häudigg. 
*479. Laimodon diadematus, Heugl.

Heugl. Beitr. t. 28, f. 1. - Bis jetzt blos im Lande der KitschNeger am Bahr el abiad, zwisehen dem $7-8^{0} \mathrm{~N}$. B. gefunden.

480. Laimodon laevirostris, Leach.

Le a ch, Zool. Miscellany V. II, t. $7 \%$ - Häufig in Schoa.

481. Barbatula (Less.) chrysocomus, Temm.

Temm. Pl. col. t. 536. - Einzeln auf Gebüsch und Bäumen längs der Ufer fliessender Bäche in Sennaar und Abyssinien.

*482. Trachyphonus (Ranzani) squamiceps, He ugl.

H e u g 1. Beitr. t. 28, f. 2. - Im Lande der Kitsch-Neger am Bahr el abiad.

483. Trachyphonus margaritatus, R ü p p.

R üp p. Atl. t. 20. -- Häufig südlich vom $18^{0}$ N. B.

\section{P i c in a e.}

484. Dendrobates (Swains on) Schoënsis, R üpp.

R ü p p. Syst. Übers. t. 33. - In Schoa.

485. Dendrobates poicephalus, $\mathrm{Sw}$ ain s on.

R ü p p. Syst. Übers. t. 34. - In Sennaar, Kordofan und Abyssinien. Das Männchen hat einen schönen carmoisinrothen Scheitel.

486. Dendrobates Hemprichii, E h re n b.

R ü pp. Syst. Übers. t. 35. - In Kordofan, Sennaar, Abyssinien und Fazoglo.

487. Dendromus (Swains on) aethiopicus, He mprich.

Häufig südlich vom $20^{\circ} \mathrm{N}$. B.

488. Dendromus (?) abyssinicus, Stanley.

In Abyssinien. Von mir nicht beobachtet.

3. Yunginat.

489. Yunx Torquilla, Lin n.

In Ägypten, Nubien, Abyssinien und Arabien. Wahrscheinlich blos im Winter.

490. Yunx aequatorialis, R üp p.

R ü p p. Syst. Übers. t. 37. - In Sehoa.

\section{CUCULDAE.}

1. Indicat orinae.

491. Indicator (L e V a ill.) archipelagicus, $\mathrm{T}$ e $\mathrm{mm}$.

Te mm. Pl. col.t.542. - In Abyssinien, in Galabat und auf dem Bahr el abiad zwischen dem $\mathbf{4 - 5 ^ { 0 }} \mathrm{N}$. B.

*492. Indicator albirostris, L e Vaill. (?)

Vom Bahr el abiad, aus dem Lande der Bari-Neger. ( $5^{0} \mathrm{~N}$. B.)

(Heuglin.) 
*498. Indicutor Prariames, Heugl.

Vom Lamde der Bari-Neger am Bahr el abiad ( $3^{0}$ N. B.).

494. Indicutor minor, Le $\mathrm{V}$ a ill.

lse Vaill. Ois. d'Afr. t. 242. - In Abyssinien und Galabat.

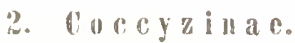

493. Centropus (Illig.) senegrelensis, Bris s.

Deseript. de l'Lgypte t. 4, f. 1. - In Unter-Ägypten in kleinen Gesellschaften.

496. Centropus Monuchus, Rü Pp.

R ii p p. N. Wirbelth. t. 21, f. 2. - In S.-Nubien, am weissen und batuen Fluss, am Athara und in Abyssinien.

497. Centropus superciliosus, Rüp p.

R ü p p. N. Wirbelth. l. o 1 , f. 1. - Südlich vom $18^{0}$ N. B.

498. Cocrystes (Glog.) glandarins, Linn.

Deser. de l'Egypte t. 4, f. 2. - In Ägypten, Nubien und Arabien, aber überall einzeln. Am haiufigsten traf ich ihn bei Cairo, Siut und Dongola.

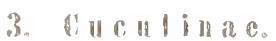

499. Oxylophus ( $\mathrm{S}$ wa inson) afer, $\mathbb{L}$ e ach.

Le $\mathrm{V}$ a ill. nis. I'Afr. 1. 200\%. Am Mahr el abiad, in Fazoglo und Abyssinien.

300. Oxylophus serrotus Ib and.

Le V a ill. Ois. d'Afr. l.208 - Wie der Vorige und einzeln bei Chartum.

* 301 . Cuculus (Iinu.) ruficollis, Heugl.

Cuculus lineutus, Swainson (?) - C. suliturius, Le Vaill. Dis. d'Afr. l. 206 (?) - Einzeln im ganzen Gebiete des Bahr el abiad.

302. Cuculus eranoms, dinn.

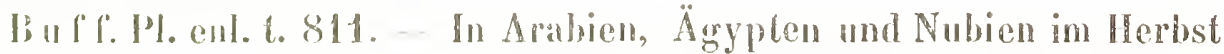
and Fröhjallr; einzelne veriprte Vügel bleiben bis Mai in Ägypten, und im Honale August habe ieh in Nubien, und bei Chartum im September schon wieder junge Vügel auf dem Rëiekzuge ins Innere Afrika's angetroflen.

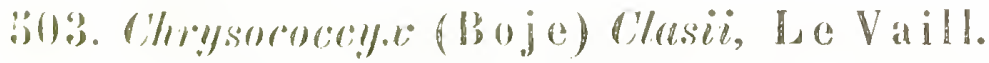

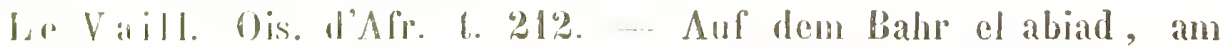
b) lauen Fluss, in dialabat und Alyssinien; nördlich bis ins Mareb-Thal.

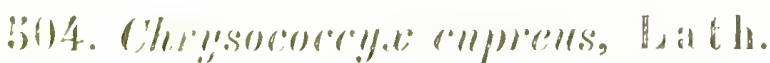

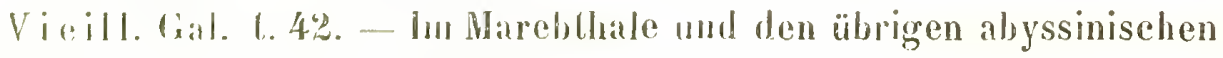
Tieflitind rern und sïdlich von liazoglo.

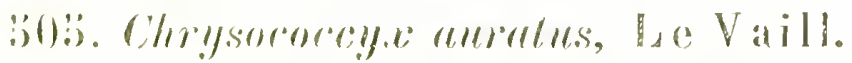

Le Yaill. Ois. d'Afr. 1. 210. - In Aloyssinien, Sennarar und am Bialie ol abiarl. 


\section{ORDNUNG. COLUMBAE (TAUBENVÖGEL).}

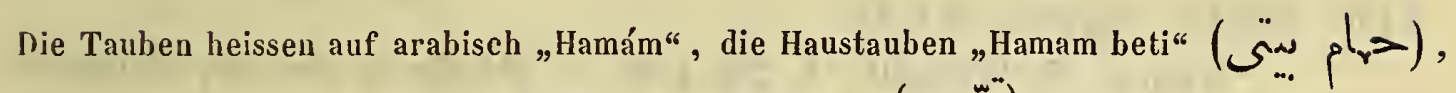
die Turteltauben „Gimmrie" $\left(\mathcal{S}^{\mathrm{g}}\right)$.

1. Co I u m b in a e.

506. Palumbus (K a u p) Livia, Linn.

Descr. de l'Egyte t. 13, f. 7. - Sturm, Fauna Deutschl. Vög. t. 12. - T e mm. Pig. t. 12. - Längs dem Nil bis ins südliche Nubien paarweise und in grossen Flügen. Nistet vorzüglich in Felsen.

50\%. Palumbus arquatrix, Tem m.

Le Vaill. Ois. d'Afr. t.264. - T e m m. Pig. t. 5. - In Flügen in den abyssinischen Wald-Regionen.

508. Palumbus guineus, Lin n.

T e m m. Pig. t. 16. - In Abyssinien, Kordofan, Sennaar, Fazoglo etc. Sowohl auf Bäumen als Felsen und Häusern. In Simehn bis auf $10,000 \mathrm{~F}$. hoch.

509. Palumbus albitorques, Rüpp.

R üp p. N. Wirbelth. t. 22, f. 1. - Auf Bäumen und Feldern in ganz Abyssinien. Im März 1853 beobachtete ich ungeheuere Flüge dieser Taube auf den Hochebenen der Provinz Woggara.

2. Peristerinae.

510. Geopelia ( $\mathrm{S}$ wain s on) humeralis, Wag $\mathrm{l}$.

C. abyssinica, L a th. - C. Waalia, B ru ce. - L e V a ill.0is. d'Afr. t. 276. - T e mm. Pl. col. t. 191. - In Abyssinien, Sennaar, Fazoglo, Kordofan und am weissen Fluss in grossen Flügen auf Hochbäumen.

511. Peristera (Swain son) chalcospilos, Wag I.

R üp p. Syst. Übers. t. 38. - In Sennaar, Abyssinien, Fazoglo und Kordofan. In ungemein grosser Anzahl in der Provinz Galabat.

512. Turtur auritus, R a y.

Col. turtur, Linn. - Temm. Pig. t. 42. - In kleinen Gesellschaften und grösseren Flügen im Frühjahr und Herbst in ganz N. 0. Afrika.

513. Turtur risorius, Linn.

L e V a ill. Ois. d'Afr. t. 268. - T e m m. Pig. t. 44. - Einzeln in Ägypten und südwärts bis Abyssinien.

514. Turtur aegyptiacus, T e mm.

Descr. de l'Egypte Ois. t. 9, f. 3. -. Sehr häufig in ganz N. 0. Afrika. 
B̈15. Turtur senegulensis, Linn.

Südwär'ts vom $21^{0} \mathrm{~N}$. B.

316. Turtur lugens, Riipp.

R ̈̈ p p. N. Wirbelth. t. 22, f. 2. - Häufig in den abyssinischen Hochläindern.

31\%. Turtur semitorqualus, R ii p p.

R ü p. N. Wirbelth. t. 23, f. 2. - Nicht selten in ganz Abyssinien, mit Ausnahme der höehsten Gebirge.

318. Turtur bronsimus, Rüpp.

R üp p. N. Wirbelth. t. 23, f。 1.-- Von Dr. Rüppell in den Thälern von Simeln beobachtet.

319. Ectopistes ( $\mathrm{S}$ wains on) copensis, Lath.

Le Vaill. Ois. d’Afr. t. 273 . - Vom $20^{\circ}$ N. B. an südlich überall mit Ausnahme der Gebirgsgegenden. Bei Abu-Harahs am blauen wlusse traf ich sie in December 1833 zu vielen Tausenden.

\section{ORDNUNG. GALLINAE (HÜHNEPVÖGEL).}

1. Ne l e itgri na e.

520. Numida (Linn.) ptilorhyncha, Li cht.

Rüpp. Syst. Übers. t. 39. - Nicht nördlich vom $16^{\circ}$ N. B. Überall in Steppen, Buschwerk und Wäldern in Ketten oft bis zu Tausenden beisammen. Von mir in Abyssinien nicht höher als etwa $8000 \mathrm{~F}$. beobachtet. Heisst auf arabisch "Didjadj of Wadi“ (د) (د) (د).

2. Poraticinas.

321. Philopuchus (?) (Swainson) ventrulis, Valenciennes.

Perdix fusec, Vieill. Gal. t. 21\%. - In bergigen und felsigen Gegenden in Kordofan, Fazoglo und namentlich häulig in der Kolla von W.-Abyssinien. In Ketten bis zn $1 \%$ und 13 Stiork.

:32?. Dhilopachus Heyi, 'T'enm.

T'emm. Pl. col.t.328. - In zahlreichen Ketten in den Bergen und Vorbergen der sinaitischen Vhalbinsel, und inn nördlichen Theile des

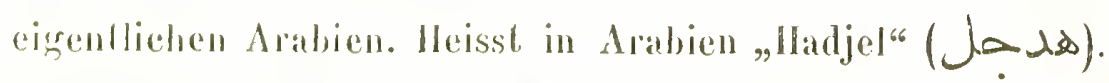

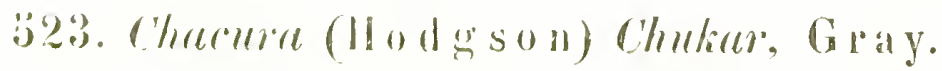

Gria y, Ind. \%ool. Vol. 1, 1. B.4. - (Chacura graeca, Briss.?) Parrweise und in grossen Ketten in den Gebirgen der sinaitisehen Halbinsel. Meisst dort "Sennat".

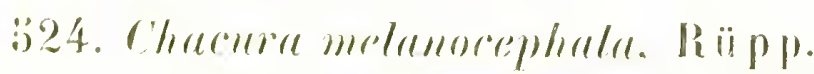

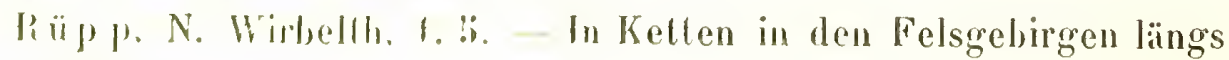
der arabischion Küustr. 
*ร2อั. Francolinus (B riss.) Francolinus, Linn.

Nach Dr. Rüppell (N. Wirbelth. S. 11) im Winter einigemal im Delta beobachtet.

526. Francolinus Erkelii, Rü pp.

Rüpp. N. Wirbelth. t. 6. - Nicht selten in den abyssinischen Gebirgen östlich vom Tana-See paarweise und in kleinen Ketten.

527. Francolinus Rüppellii, G. R. Gray.

Perdix Clappertonii, Rüpp. Atl. t. 9. - Arahisch „Didjadj el gesch“ (دحاج القشى). - Häufig in grossen Ketten in Kordofan, Sennaar, Fazoglo und Abyssinien.

*28. Francolinus icteropus, He $\mathrm{gl}$.

Heugl. Beitr. t. 29. - Paarweise auf den Gebirgen von Simehn in Abyssinien gefunden.

529. Francolinus gutturalis, R ï p p.

$R$ üpp. Syst. Übers. t. 40. - Paarweise in den abyssinischen Gebirgen.

530. Francolinus pileatus, A. S m ith.

S mith, Ill. of Sauth-Afr. t. 14. - In Schoa.

วั31. Francolinus rubricollis, Rü p p.

R üp p. Atl. t. 30..- In Ketten und paarweise an den Abfällen von Ost-Abyssinien.

อั32. Coturnix communis, B o n n at.

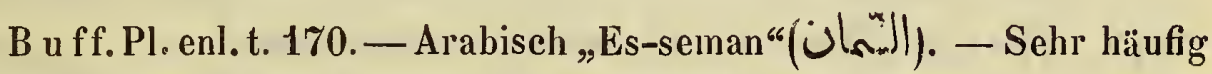
im Herbst an der ägyptischen Küste, zieht aber über den Winter südlich; in Abyssinien um den Tana-See traf ich sie häufig im Januar und März, ebenso im November in Kordofan; im Frühjahre bis Mai in Ägypten und Arabien.

*533. Coturnix crucigera, H engl.

Heugl. Beit. t. 30. - Blos ein Individuum am Bahr el abiad bei den Bari-Negern zwischen dem 4-50 N. B. gefunden.

*534. Coturnix strictus, C uv. (?)

Tem m. Pl. col. t. 82. - Bei Ben-Ghasi eingesammelt.

3. P t e r o c li il a e.

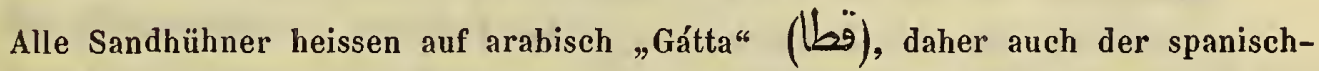
maurische Name „Al-cháta“.

535. Pterocles (Te m.) Alchata, Lin n.

Buff. Pl. enl. t. 10马 und 106, - In der lybischen Wüste bei BenGhasi, Tunis ete. etc. 


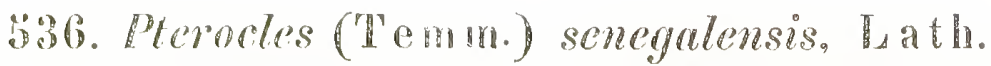

Pt. gnttatus, Lieh t. - Buff. Pl. enl. t. 130. - Te m m. Pl. col. t. 345. - In Wüsten und Steppen von Unter-Ägypten an südlich überall gemein, oft in ungeheuren Flügen.

333. Plerocles exustus, T emm.

PI. col. t. 394 und 360. - In ganz N. 0. Afrika und Arabien.

äs8. Plerocles coronatus, Licht.

Temm. Pl. col. t. 339 und 340. - In Nubien und Kordofan in grossen Flïgen.

ร3. P. Procles Lichtensteiniu, Temm.

Temm. PI. cot. 1. 33 š und 361. - Wie der Vorhergehende.

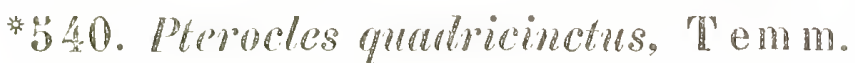

Vieill. Gal. pl. 2280. - Pr. pasciatus, Lieht. (?) - Blos einzeln oder in Gesellschaften von 3-6 Stücken in den Wald-Regionen von Sennaar und Abyssinien.

:41. Pterocles guthuralis, A. Smith.

Smith, III. of South-Afr. t. 3 und 31.... Am Mareb, in der Umgegend von Adoa und Axum, wind in Sehor.

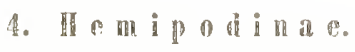

* 4 4. Ortyrelos (Vi e ill.) isabellimes, Heugl.

He ugl. Beitr. t.31.- Hemipodiusmivosas, $\mathrm{S}$ w a in s. (?) - H. Meiffrenii. Temm. Pl. col. t. 60, f. 1 (?) - Einzeln und parweise in den Steppen von Kordofan.

*

Hemipod. Acedoydromus, T' e mm.- In der Provinz Seherkic in Unter$\ddot{A}$ sypten auf Kee-Wiesen beobachtet, olme eingesanmelt werden zu können; in der Gegend von Benghasi und Tripolis häufig eingesammelt.

\section{ORUNUNG. CURBORES (LAURVÖGEL).}

I. St t athion in it:

344. Stmuthio Cremelus, ILinn.

Araluscht "Nótamelı" (dob).

Paarweise in grossen Gesellschaften in Ägypten, Nubien, Kordofan, der abyssinischen Kïste und längs des blanen und weissen Flusses. Am laïufigsten trat ich ilı in Ost-Sennatr und einzelnen Theilen Kordofans, Hamentlich bei den Kababisci und Dar-Ifammr. - Merkwärdig ist das liaden der Strausse in Meere; an heissen Tagen nänlich sieht man oft grosse Truplen an Siundbinken und flachen Ufern weit, vom Lande cufferml, his $1 m$ den oherhals in Wasser stehend, stundentang an den ahyssinischon Kiistentiudlerm. In gebirgigen Gegenden kommt der Strauss ure vor, aurla reht er Steppenlandschaft der freien Wüste vor; 
er ist, wie überall, äusserst scheu und furchtsam und blos bei Stürmen und Gewitterregen lässt er sich leicht erschleichen.

Die Kraft des Thieres ist ausserordentlich und es setzt sich - in die Enge getrieben - mit seinen Füssen fürchterlich zur Wehre. In der lybischen Wüste und in Mittel-Ägypten nordwärts bis Cairo habe ich nie selbst Strausse beobachtet, doch versicherte mich unter andern ein sehr zuverlässiger Jäger, der Prinz Halim Pascha, dass er, einige Tagreisen von Cairo entfernt, sogar frisch zerstörte Brutplätze derselben gefunden.

\section{0 t i din a e.}

545. Houbara (B o n a p.) undulata, J a c q.

Otis Houbara, Lin n. - Vi eill. Gal.t.22\%. - Naum.V.D.t.170.Arabisch „Hubara“ ( $\mathrm{j} h$ ), wie alle übrigen Trappen im Süden. - Nach R üp pell einzeln in N. 0 . Afrika. In der lybischen Wüste ist sie nicht selten, in Ägypten selbst habe ich sie dagegen nie gesehen und ich fand nur einmal in der Nähe des Djebel Atága am rothen Meere frische Fährten, die möglicher Weise diesem Vogel angehörten.

546. Houbara Nuba, R ü pp.

Otis Nuba, R üpp. Atl. t. 1. - In den Steppen des südlichen Nubien und in N.-Kordofan ziemlich häufig.

547. Lissotis (Reichenb.) melanogaster, Rüp p.

R üp p. N. Wirbelth. t. \%. - R üpp. Syst. Übers. t. 41. - Sehr häufig am Tana-See in Abyssinien, in den Steppen von 0st-Sennaar (bei Djebel Atesch, Kedaref, in Taka etc.), am Bahr el abiad und einzeln in den Kolla-Ländern von W. und N.-Abyssinien.

548. Lissotis senegalensis, Vieill.

Otis Rhaad, Lath., - O. Barrowii, Gr a y. - R ü pp. Mus. Senkenb. Vol. II, t. 15. - Häufig in Schoa.

*549. Lissotis semitorquata, $\mathrm{H} \mathrm{e} \mathrm{u} \mathrm{g}$.

H e u g l. Beitr. t. 32. - Einzeln in den Steppenländern der SchillukNeger am Bahr el abiad.

*550. Lissotis afra, Lath.

L. afroides, A. Smith, Birds of South-Afr. t. 19. - In Fazoglo und Beni Schangollo, wahrscheinlich auch in den Steppen von Ost-Sennaar.

551. Eupodotis (Le ss.) Arabs, Linn.

R üpp. Atl. t. 16. - Häufig in Sennaar, Kordofan, Süd-Nubien und um den weissen Fluss und Sobat. Soll einzeln in Ägypten vorkommen.

A $\mathrm{nm}$. Wenn ich nicht irre, hat Seine Hoheit der Herzog Paul ron W ürtemberg auch Otis Caffra oder Otis Ludwigii Rüpp. in Sennaar oder in Kordofan gefunden. 
*33:. Otis Tetrax, Isinn.

$\mathbb{N}$ a um. V.D.t. 169. - Einzeh im Winter an der ägyptischen Küste, rorzüglich bei Pelusium, el-Arisch ete. Hänliger erhielt ich ihn un die Syrten und im Innern des Cyrenaica.

\section{ORDNUNG. GPALILATORES (WADVÖGEL).}

\section{A. COA}

1. Oedicneminite.

333. Dedicnemus (T'emm.) erepoitrons, L i n $\mathrm{n}$.

Bu uf. Pl. cnl. t. 919. - Nanm. V. D. t. 17\%. - Arabisch „Karawán chéti"(

落4. Dedicnemus uffures. Rïpl.

R ï pp. Sysí. Übers. t. 秘. - An der abyssinischen Küste und an den Ufern des Bahr el abiad, südlich rom $6^{0} \mathrm{~N}$. 0 .

\section{C I l s orinas.}

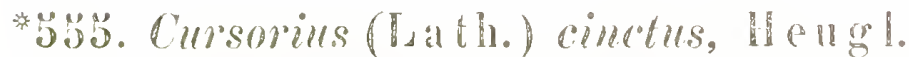

He ugl. Ibeitr. t. 33. - Am Bahr el abiarl, unterm $4^{0}$ N. B. aufgefunden. 356. Cursonins enropuens, Is a th.

C. isabellims, A u et.-B uff. [Pl. enl. t. 795 . - N a um.V.D.t.171. -

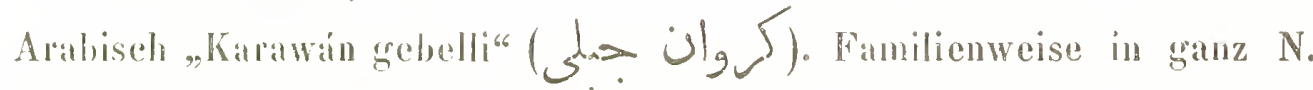
0. Afrika mit Ausuadme sler Gebirgsgegenden; in Steppenlandsehaft und Wiisten.

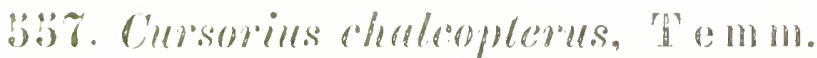

Te m m.1. Pl. eol. (. 2998. - In kleinen Familien am blauen Fluss und in Ost-Sennaatr.

3:58. Cursorius semegulemsis, Licht.

C. 'Temminclio S wains. Birds of West. Afr. If. t. 24. - In Flïgroll an Tant-See in Abyssinien.

Ost-Sennatat und ann Timat-See.

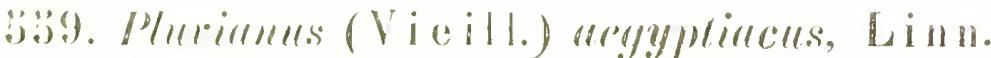

Irescer. de l'tegypte t. i, r. 4. - - Arabisch "teer el temsach"

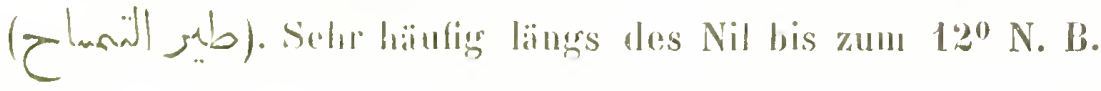

3. I) ha a a it ri in ac.

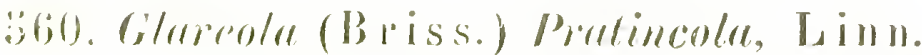

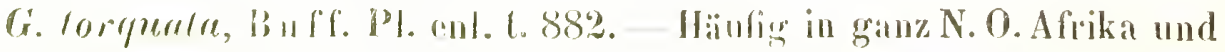

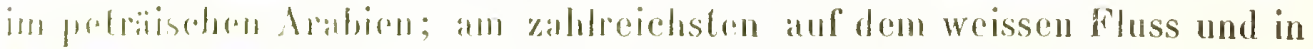

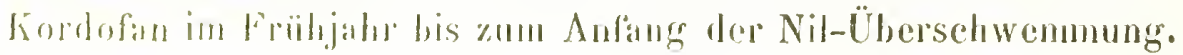


561. Glareola limbata, R üpp.

Rüpp. Syst. Übers. t. 43. - In Abyssinien und an der arabischen Küste; ein Exemplar meiner Sammlung ist auch in Nubien bei Dongola eingesammelt worden.

*562. Glareola melanoptera, No r d m a n n.

G. Nordmanni, Fis ch er. - G. brachydactyla (?) - In kleinen Familien auf Feldern und Wiesen in Ägypten und Nubien. Am häufigsten traf ich sie im October 1851 im Fajum in Mittel-Ägypten.

563. Vanellus (B riss.) cristatus, May er et Wolf.

B uff.Pl. enl. t.242. - N a u m. V.D.t.179. - Im Winter und Frühjahr in grossen Flügen und einzeln in Ägypten und Arabien.

564. Vanellus coronatus, Lin $\mathrm{n}$.

B u ff. Pl. enl. t. 800. - Nach R üp p ell ziemlich häufig in Nubien.

56ว. Vanellus Villotaei, S a vign y.

V. leucurus, Licht. -- Descr. de l'Egypte t. 6, f. 2. - In Nubien, Sennaar, Kordofan, seltener in Ägypten, wo ich ihn blos im Fajum antraf.

*566. Vanellus pallidus, He ugl.

H e u g l. Beitr. t. 34, f. 2. - Vanellus gregarius, P all as (?) In grösseren Flügen in den Steppen von Ost-Sennaar und Taka.

*567. Vanellus macrocercus, H e $\mathrm{gl}$ l.

V. gregarius $\mathrm{Pall}$ as (?) - H eugl. Beitr. t. 34, f. 1. - An den Ufern und in den Steppen um den Bahr el abiad südlich vom $10^{\circ} \mathrm{N}$. B.

5้68. Lobivanellus (Strickland) melanocephalus, Rüpp.

Rüp p. Syst. Übers. t. 44. - In kleinen Gesellschaften in den abyssinischen Gebirgslandschaften.

569. Lobivanellus senegalensis, Lin 1.

Vanellus lateralis, A. Smith. III. of South-Afr. t. 23. -- In Abyssinien und am Bahr el abiad.

*570. Squatarola (Cuv.) helvetica, B r is s.

$\mathrm{Nau}$. V. D. t. 178. - Vanellus varius, Briss. - V. melanogaster, B e chst. - Im Winter einzeln in Unter-Ägypten.

571. Hoplopterus (Bonap.) spinosus, $\mathrm{H}$ a s s elq.

Descr. de l'Egypte t. 6, f. 3. - Heisst auf arabisch "Siqsaq" (سقتاق). - Gemein in ganz N. 0. Afrika.

572. Sarkiophorus (Stri ckland) pileatus, L in n.

B u ff. Pl. enl. t. 834. - In kleinen Gesellschaften in den Steppen von Nubien, Kordofan und Sennaar. 
*373. Charadrius pluvialis, Linn.

Ch. auratus, Sukow. - Naum. V. D. t. 173. - Im Winter in Gesellschaften an der ïgyptisehen Küste.

374. Charadrius melanopterus, Rï ï p p.

R ï p. Atl. t.31. - Hitufig in Abyssinien, nach Dr. R ü p p ell auch in Nubien.

šrb. Aegialites (B o je) cantirnus, L ath.

N a u m. V. D. t. 176. - Im Winter in Ägypten und Nubien. 376. Aegialites hiaticula, Linn.

N a um. V. D. t. 189. - Deser. de l'Egypte 1.14, f. 1. - Im Winter in Unter- ̈̈gypten.

*397. Acgialites hiaticuloides, $\mathrm{Heugl}$

Heugl. Beitr. t. 35, f. 2. - Einzeln in Abyssinien und Galabat.

* 38 . Aegialites auritus, H eug $\mathrm{l}$.

Am Bahr el abiad, zwisehen dem $4-6^{0}$ N. B.

3\%9. Acgialites minor, Mayer et Wolf.

N aum. V. D. t. 1\%\%. - Buff. Pl. enl. t. 921. - Nicht selten in Ägypten, Nubien und am rothen Meer.

* 80 . Aegialites albifions, Cav. (?)

Von Herzog Paul von Würtemberg am blanen Fluss eingesammelt.

*81. Aegialies cinereocollis, He $\mathrm{ng}$ l.

Heugl. Beitr. t. 3\%, f. 1. - Aeg. bitorqualus, Licht. (?) Einzeln an den abyssinischen Gebirgswässern.

¿38. Acgialites imlicus, Lath.

Nach R ïppell zienlich häufig am rothen Meer.

38\%. Aeginlites reotfroyi, $\mathrm{W}$ ag $\mathrm{l}$.

Wagl. Syst. Avimm. Sp. 19. - Wie der Vorhergehende.

* 84 . Aregialites longipes, Ill eugl.

II ("I I I. Beitr. I. 3:, f. 3. (Vielleicht identiseh mit dem Folgenden.) - Nicht selten in Nubien und am Bialre abliad.

:38. Aegialiles peruntius, 't' emm.

Temm. Ir. col. 1. 18\%. - Naclı Rüppell in Ägypten.

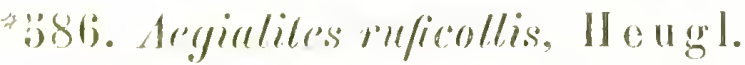

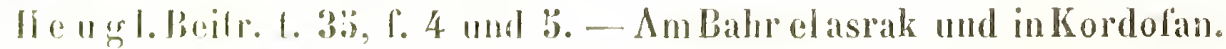

1. Ui incilinis.

387. Eudromias (Boje) Morimellus, Linn.

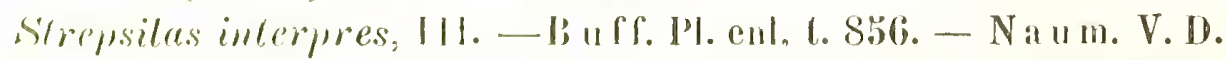
t. 176. In Winter in Ägypten und ant rothen Meer. In December $18: 31$ hooblaclitele ich zrosse Fligge dieser Art zwisehen Sakkara und deon Fajum in der Wiiste. 
*588. Eudromias asiaticus, P all.

Charadr. jugularis, W a g l. - Im Winter an den Küsten des rothen und mittelländischen Meeres.

*589. Strepsilas (1llig.) interpres, Linn.

Morinella collaris, M a y er. - N a u m. V. D. t. 180. - Im Winter bis Monat Mai in kleinen Flügen am mittelländischen Meer. Verlässt Ägypten im vollständigen Hochzeitskleid.

\section{Ha emat op odinae.}

590. Haematopus Ostralegus, Linn.

B u ff. Pl. enl. t. 929. - N a u m. V. D.t. 181. - Einzeln im Winter an der ägyptischen Küste. Das ganze Jahr über gemein am rothen Meer.

591. Haematopus niger, $\mathrm{Cuv}$.

Nach Dr. R üppell einmal auf der Insel Dahlak im Archipel von Massaua erlegt.

\section{B. A R D E I D A E.}

\section{Gr u in a e.}

592. Grus (Pall.) cinerea, Bechst.

$\mathrm{N}$ a u m. V.D.t. 231. - B u ff.Pl. enl. t. 769. - Überwintertin ungeheuren Schaaren am weissen und blauen Fluss und in Kordofan; im Frühjahr und Herbst auf dem Durchzuge in Nubien und Ägypten.

ร93. Grus carunculata, Lath.

Nach Dr. Rüp pell einzeln in Schoa.

594. Anthropoides (Vieill.) Virgo, Linn.

B uf f. Pl. enl. t. 241. - N a u m. V. D. t. 232. - Arabisch „Raho“ (s) (s). - Im Winter in unzähligen Schaaren am weissen und blauen Fluss und in Kordofan, wo er vorzüglich von Durrah und Dochen lebt.

595. Balearica (Bris s.) pavonina, Lin n.

B u ff. Pl. enl.t. 265 (?) — Arabisch „Gharnúb“(غرزوب). - Ich kann nicht bestimmen, ob diese Art wirklich verschieden von B. regulorum, vom Caplande ist.

Der Kronenkranich lebt in grossen Schaaren am Tana-See in Abyssinien, am ganzen weissen und blauen Fluss und in Kordofan. Im Sommer scheint er sich mehr nach Süden zu ziehen, doch fand ich einzelne brütende Paare schon in der Nähe der Schilluk-Inseln zwischen dem 12 u. $13^{0}$ N. B. im November. Weiter im Süden scheint er noch später zu brüten, da ich junge Vögel im März vom Sobat erhielt, die kaum zwei Nonate alt sein konnten. 
2. A r d c in a c.

Die grossen Reiher heissen auf arabisch „Balaschán" (نlit).

396. Ardere cincrera, I, inn.

B u f f. Pl. enl.t. 7:3.\%. - N a um. V. D. t.220. - Nicht selten in ganz N. O. Afrika.

397. Arder morpmert, Linn.

Poufl. Pl. enl.t. 788.- N a um. V. D. t. 221. - In ganz N. 0. Afrika cinzelu, seheint auch hier zu hriten. Im Herbst 1833 beobachtete ich einen mrossen Flug dieser Vögel bei den Ruinen von Sobah am blauen Fluss.

39S. Ardere Goliath, llu iip.

R ïpp. Atl. t. 26. - Einzeln am Tana-See, und am weissen und blauen Fluss. (Kommt auch in Sïd-Afrika am Port Natal vor.)

b̌ 99. Arlea alrivollis. Vicill.

A. nigrirollis, Anet. - A.Smitli. Ill. of South.: Afr. t. 86. Wagl. Syst. Avium Sp. 1. - - Nicht selten in Abyssinien und Ost-Sennaar. Hät sich meist auf freiem Felde und nicht am Wasser auf.

\$300. Eyprelta (Briss.) rlbr, Hinn.

Nach Dr. Ri iipell in Unter-Ägypten.

B01. Egretla oricululis, Gin ay.

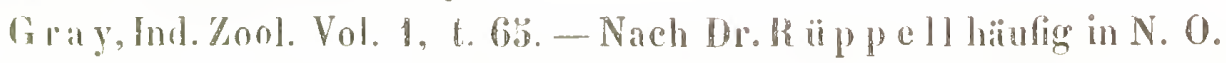
Afrika. Ireh kenne den Untersehięl dieser von der vorhergehenden Art nicht, und alle von mil in Ägyplen, Nubien, Abyssinien und am Bahr el abiad

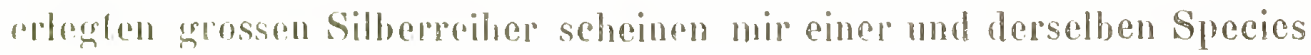
al1zugetiören.

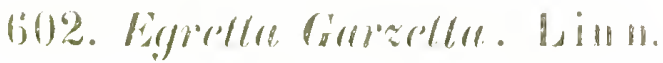

Na 11 11. V. 1). 1. 2225. - Irälig in gan\% N. U. Afrika, vielleicht in

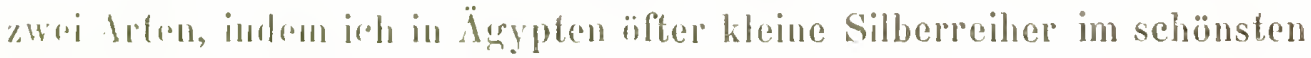
Schmolukleide erfogte, wetehe rein weisse lris hatten, und bei denen die

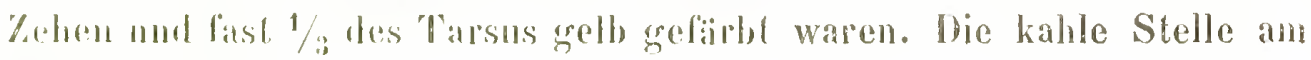
Ange ist violett.

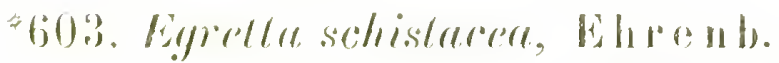

Ehrout. Symb. 1. 6 A. ulbicollis, Vieill. Gal. t. 233 (?) - An wen lfem des rothen Meeres.

6014. Egrolla Alarirostris, T'e mm.

Wa gl. Syst. Arium. Sp. 9. - In Kordofan.

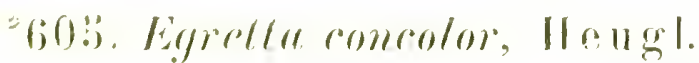

lleugl. Brile. t. 39. - Hos ein Rxemplar am Sobat-Flusse einm gesedumelt. 
606. Egretta ardesiaca, W a gl.

W a gl. Syst. Avium Sp. 20. - Am Bahr el abiad.

607. Buphus (B oj e) russatus, Wa g l.

W a gl. Syst. Avium Sp. 12. - B. bubulcus, S a vign y, Deser. de l'Egypte t. 8, f. 1. -- Arabisch „Abu Gördán“ (الو قودان).

608. Buphus coromandelicus, Li cht.

Diese beiden Reiher hält Dr. Rü pp ell für unbezweifelt verschiedene Arten, was ich nicht widersprechen kann, da ich keinen ägyptischen zum Vergleiche bei Handen habe. Ich fand sie sehr häufig in Ägypten, Nubien, Abyssinien und auf dem weissen Fluss. B. bubulcus brütet in Unter-Ägypten in grossen Gesellschaften in Mimosen-Wäldern im Monate August.

609. Buphus ralloides, Scop.

Ard. comata, Pallas. - Buff. Pl. enl. t. 348. - Naum. V. D. t. 224. - Einzeln in ganz N. 0. Afrika. Am häufigsten traf ich ihn in den Monaten Juni und Juli zwischen Assuan und Dongola am Nil.

*610. Buphus leuconotos, W a gl.

W a gl. Syst. Avium. Sp. 33. - Sehr einzeln an blauen Fluss.

*611. Buphus griseus, Buff.

B u ff. Pl. enl. t. 908. - A. scapularis, Illig. - Nicht selten in Nubien und am blauen und weissen Fluss. Ich glaube ihn auch in Abyssinien beobachtet zu haben.

*612. Ardeola (Bonap.) minuta, Linn.

B u ff. Pl. enl. t. 323. - Na u m. V. D. t. 227. - Einzeln im Herbst in Nubien, nach Dr. Rüppell auch in Abyssinien, wahrscheinlich auch in Ägypten.

*613. Ardeola pussilla, He ug l.

A. cancrophaga, S mith, Ill. of South-Afr. t. 91 (?) - Einzeln am Ufer der Schilluk-Inseln.

614. Botaurus (B riss.) stellaris, Linn.

Buff. Pl. enl. t. 789. - Naum. V. D. t. 226. - Im Wintel und Frühjahr in Ägypten, am rothen Meer und in Abyssinien; hier vielleicht Standvogel.

615. Scotaeus (Ke y s. et B las.) Nycticorax, L in n.

B u f. Pl. enl. t. 758. - N a u m. V. D. t. 225. - In ganz N. 0. Afrika. Im Süden wohl blos im Winter. Im März und April 1853 begegnete ich grossen, offenbar im Wandern begriffenen Flügen am Tana-See in Abyssinien.

*616. Scotaeus guttatus, Heugl.

Am Sobat-Fluss unterm $9^{0} \mathrm{~N}$. B. 
*617. Seotuens (?). . . (?).

In kleinen Flïgen an Tana-See in Abyssinien. Kleiner als Sc. Nycticorax, dunkel schiefergrau, nit orangegelben Weichtheilen. Konnte teider nicltt erlegt werden.

618. Sropus (Bpiss.) Umbrettre Linn.

Paarweise in Semaar, am weissen Fluss und in Abyssinien. Ziemlich häufig.

619. Plalulere leucorodin, Linn.

B uff. Pl. enl. t. 40 马. - N aum. V. D. t. 231. - Arabisch „Abu Málaga" (dëlos gl). - Längs des ganzen Nil; im Winter sehr hïufig in Unter-Ägyten. - Vielleicht zwei Arten.

620. Platulea temirostris, 'T'emm.

Häufigg am blauen und weissen Fluss und in den Sümpfen von Kordofall.

*621. Platalea. . . (?).

In Gesellschaften im südlichen Nubien und lïngs des Bahr el abiad.

3. Ui con i I a e.

622. Amestomus (BBon nat.) lamelligerus, Illig.

T' emm. Pl. col. i. 236. - Häufig am Tana-See, dem blanen und weissen Fluss und in grossen Sehaaren in den Fulen von Kordofan.

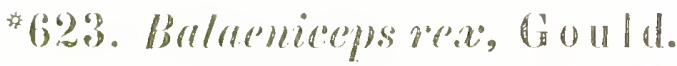

If eu gr l. Beitr. t. 40. - Selten, namentlich während der Regenzeit, am weissen Fluss in Lande der Nuer-und Kitseh-Neger, hïufiger an den Flïssen Far, Nam und Niebohr westlich vom Bahr el abiad; in grossen Schaturen inn Schilf und Ambatselt-Gebüseh, auf dem er nistet.

624. Dromes Ardeole, Pa y kul.

'I'e mm. Ir. enl. t. 36\%. - In kleinen diesellschaften an Ufer des mollen Heeres.

(i2:). Cicomina albu, Li inn.

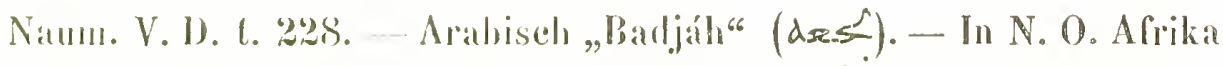
als Zugvognd. Überwintert in Durrah-Feldern in Ost-Sennaar in grossen Sicharm.

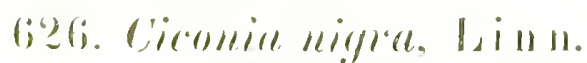

liuff. P. (ml. 1. 399\%.- Na um. V. D. t.2209- - In ganz N. O. Afrika rinzoln und in hloinen Gesellschalten.

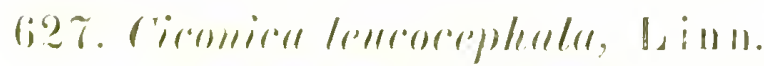

liuf'f. Pl. enl. 1. 906. In Alyssinien und an Bahre al astak und abial paratione. 
628. Sphenorhynchus (H e m p r.) Abdimii, Li c ht.

R üp p. Atl. t. 8. - Arabisch „Sinbila“ (w w w ) - Überwintert in Abyssinien; in Sennaar, Nubien und Kordofan erscheint er als Verkünder der Regenzeit im Mai und Juni, und verschwindet, nachdem die Jungen flügg geworden sind, im November gänzlich.

629. Mycteria (Li n.) ephippiorhyncha, T e m m.

R üpp. Atl. t. 4. - Einzeln in Abyssinien und Galabat, häufiger auf dem blauen und weissen Fluss und in den Fulen von Kordofan.

*630. Mycteria senegalensis, $\mathrm{Sh}$ a w.

Am Bahr el abiad und in 0st-Sennaar.

631. Leptoptilos (L e ss.) Argula, L inn.

Temm. Pl. col. t. 301. - Arabisch „Abu-Sen“ (إو سن). - Nicht selten südlich vom $18^{\circ} \mathrm{N}$. B. In grossen Flügen zu vielen Hunderten fand ich ihn im April 1853 in den Quellenländern des Rahadflusses.

4. Tantalinae.

632. Tantalus Ibis, Lin n.

Buff. Pl. enl. t. 389. - Das ganze Jahr über in Sennaar und am weissen Fluss. Während der Regenzeit nördlich bis Ober-Ägypten.

633. Ibis (B r is s.) aethiopica, Lath.

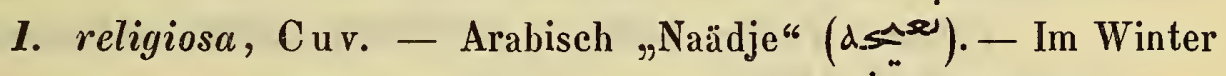
ungemein häufig am Tana-See in Abyssinien; am blauen und weissen Fluss, in Nubien und Kordofan. Brütet nordwärts bis gegen Wadi Halfa, im Juli, - sehr häufig aber bei den Schilluk-Inseln auf dem weissen Fluss im September und October.

634. Harpiprion (W a g l.) carunculatus, R ü p p.

R ü pp. N. Wirbelth. t. 19. - In grossen Schaaren im Frühjahr auf Wiesen und Feldern in Abyssinien; geht bis 10.000 Fuss hoch. Nach Rüppell während der Winterregen an der abyssinischen Küste.

635. Harpiprion Hagedash, S p a r r m a n n.

Vieill. Gal. t. 246. - Nicht selten am blauen und weissen Fluss in Steppenlandsehaften.

636. Geronticus (Wagl.) comatus, Ehrenb.

$R$ üp p. Syst. Übers. t. 45. - Im Winter an der abyssinischen Küste; im Februar 1833 fand ich ihn auf der Hochebene von Woggara in grossen Flügen, gemeinschaftlich mit $\boldsymbol{H}$. carunculata. Er scheint in Abyssinien zu brüten; der junge Vogel hat ein schmutzigweisses ganz befiedertes Gesicht, und erst am Halse geht diese Farbe nach und nach in die schiefergraue Farbe der Halsbasis über. 
63\%. Fulcincllus (Ray) igneus, Gmel.

Ibis Falcinellus, Vieill. Gal.t. 301. - In ganz N. 0. Afrika vielleicht in zwei sich sehr ähnlichen Arten. - Meine Exemplare von Abyssinien und vom Bahr el abiad sind um $1 / 4 \ldots 1 / 5$ kleiner als die curopäischen.

\section{SCOHOPMCIDAN}

638. Numenius (Dha y) Arquatu, Lath.

lb uff. Pl. enl. t. S18. - Nach R üppell häufig in Unter-Ägypten und an der abyssinisehen Küste im Winter.

639. Numenius temurostris, Vieill.

Savi, Orn. tose. II. p. 324. - Im Herbst und Frühjahr im Durehzuge längs des Nil. In Chartum erscheint er schon Ende August und Anfangs Seplember, und im April auf der Wanderung. Bei Alexandria im April in der Wüste in grossen Schaaren beobachtet.

640. Numenius phueopus, Lath.

B uff. Pl. enl. 1. 842. - Im Winter in kleinen Gesellschaften längs des Nilstromes.

641. Numenius syngenieos, v. d. Mïhle (?)

Ein Exemplar vom Cap Rasat.

642. Glottis cheloropus, Nil so it.

Totanus glotlis, Auct. - Im Winter häufig, im Sommer einzelner in ganz N.0. Afrika.

643. Totumes (Ray) stugnutilis, Beehst.

Bo u f. Pl. enl. t. 876. - Einzeln in ganz N. 0. Afrika bis Kordofan und Alyssinien. Ob er im Sommer beibt, kann ich nicht bestimmt versichern, doch erlegte ich inn Ende April in Galabat im vollständigen Sommerkleid.

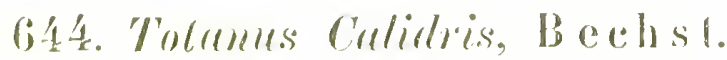

beser. de l'Egypte t. $6, f .1$. - In grossen Flügen in ganz N. O. Afrika vom lfertost bis linde Hai.

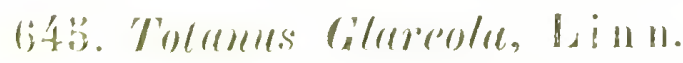

Deser. de l'Esgyte 1. 14, f. 2. - Wie der Vorhergehende, cinzeln auch den wanzen Sommer über.

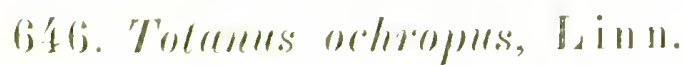

13 uff. I'l. col. 1. 843. - Wie lie Vorhergehenden, aber eimzehner.

6.87. Acliris (boje) hypoleneos, Linn.

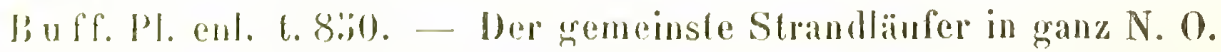
Afrika, doch ist re wie atle übrigen im Sommer seltener als zur Zungeit. 
643. Limosa (B riss.) aegocephala, Linn.

L. melanura, Leisl. - B u ff. Pl. enl. t. 874. - Vom Herbst bis Ende Frühlings in ungeheuren Flügen längs des Nilgebietes, vorzüglich aber in den Kordofanischen Sümpfen. Verlässt Ägypten im April und Mai im vollkommenen Sommerkleid.

649. Machetes (C u v.) pugnax, I, i n n.

B u ff. Pl. enl. t. 305. - Sehr häufig in ganz N. 0. Afrika vom August bis Mai. Im August 1852 traf ich bei Dongola viele männliche Vögel im Prachtkleid.

650. Calidris (Illig.) arenaria, L i n $\mathrm{n}$.

$\mathrm{N}$ a u m. V. D. t. 182. - Im Winter in kleinen Gesellschaften in Unter-Ägypten.

651. Tringa (L i n n.) subarquata, G ül denst.

B u f. Pl. enl. t. 851. - Im Winter in Ägypten und am rothen Meer.

652. Tringa Cinclus, L i n n.

Tr. alpina, I, in n. - Tr. Schinzii, B r e h m. - B u f. Pl. enl. t. 852. - In Ägypten und an rothen Meer vom October bis Ende Mai. Verlässt die Nordküste von Unter-Ägypten im vollkommenen Sommerkleid.

653. Tringa Temminckii, L e is l.

Tr. pusilla, Bechst. - Te mm. Pl. col. t. 41. - In ganz N. 0. Afrika südlich bis zum $10^{0} \mathrm{~N}$. B. beobachtet. Bleibt wahrscheinlich theilweise den Sommer über hier zurück.

654. Tringa minuta, L e is l.

Im Winter selten in Ägypten und am rothen Meer.

655. Recurvirostra Avocetta, Linn.

B u f f. Pl. enl. t. 353. - Vom Herbst bis Frühjahr in N. O. Afrika. Sehr häufig zuweilen in Kordofan und am rothen Meer.

656. Himantopus (B ris s.) vulgaris, B e c h s t.

B u f f. Pl. enl. t. 878. - Das ganze Jahr über in N. O. Afrika, südlich bis Kordofan.

657. Rhynchaea (C u v.) variegata, Vi eill.

Descr. del'Egypte t. 14. - R. bengalensis, Linn. - Das ganze Jahr über in Ägypten, wo sie im Mai brütet. Im April 1853 schoss ich ein Exemplar in der Kolla West-Abyssiniens.

658. Ascalopax (Ke y s. et Bl a s.) Gallinula, Lin n.

Vom Herbst bis Mai nicht selten in Unter-Ägypten. Sie scheint auch dort zu brüten.

659. Ascalopax Gallinago, Lin n.

B uff. Pl. enl. t. 883. - Im Herbst und Frühjahr in ganz N. 0. Afrika. 


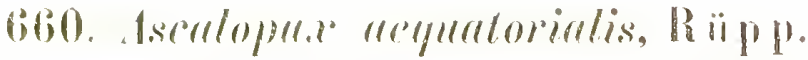

In Abyssinien. lol, fand sie häufig im Winter auf den Gebirgen von Simphn an Bächen.

*631. Asculopur major. Gimelin.

Im Winter einzeln in Unter-Ägypten.

662. Sionlopure rusticolu, Tinn.

Buff. Pl. enl. t. 88:. - Im Mïrz einzeln in Gärten und Busehwerk bei Alexandria und Rosette.

\section{PALADEDEDAF。}

663. Preron africana, Is inn.

Swa ins. Zool. Illustr. Ser. II, t. 6. - - Gemein atm Tana-See in Abyssimien, auf dem Bahr el abiad zwischen dem $7 \ldots 9^{n}$ N. B., seltener in Fazoglo.

\section{R. BALHIDAE。}

6064. Crex protensis. Hechst.

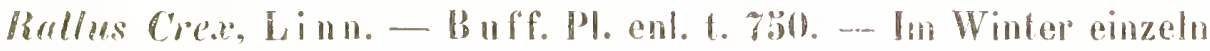
in $\ddot{A}$ ingleten und Arabien.

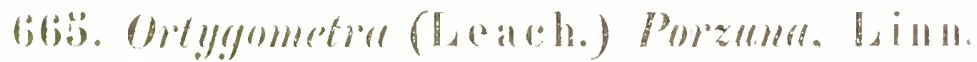

Einzeln in $\ddot{A} g y p t e n$. Abyssinien und Sudan. Ob. Standvogel, kann ich nichlit atugeben.

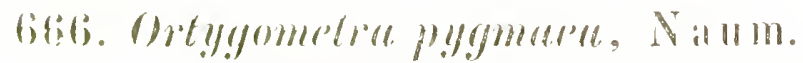

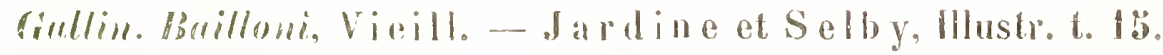

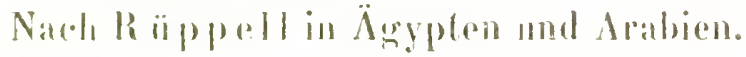

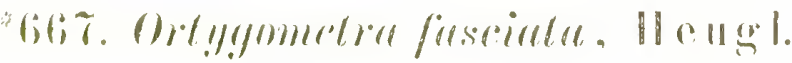

II 11 m. Broilr. 1. 37. - Am weissen Fluss 4:" N. B.

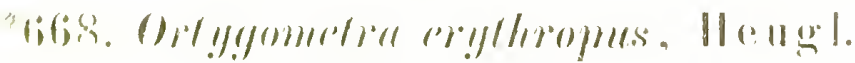

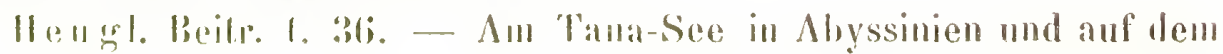
bialla al alliad.

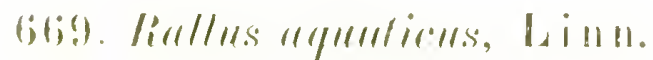

lon Winlar rinzeln in Unter-ägypten.

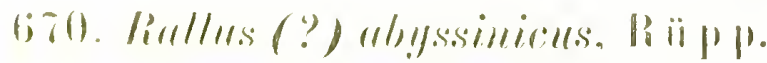

Rii pp. Srst. Ühors. 1. 46. - Gemein in Abyssinien. Ein Exemplar

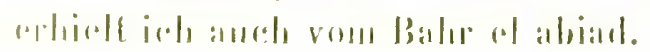

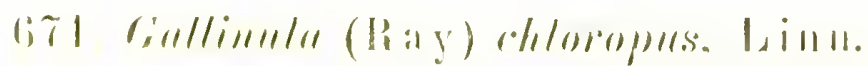

Buff. P. rol. 6.877. - Tn Winter in Ägyplen mud Arabien. 
672. Porphyrio (Briss.) aegyptiacus, He ug l.

$P$. hyacinthinus, Te mm. (?) - Gemein den Sommer über in UnterÄgypten, vorzüglich in den Seen von Etku, Damiette und in Reisfeldern. Wenn ich mich recht erinnere, fehlt er aber dort im Winter. Nir scheint der ägyptische Porphyrio von der südeuropäischen und der in Algerien vorkommenden Art versehieden, da keine Beschreibung ordentlich auf ersteren passt, doch mangeln mir Exemplare des wahren $\boldsymbol{P}$. hyacinthinus, um genaue Vergleichung anstellen zu können; der meinige ist nie blaurückig, sondern der Hinterkopf und Rücken sind mehr pistaziengrün gefärbt; ich habe diese Art hier unter dem Namen $\boldsymbol{P}$. aegyptiacus aufgestellt.

673. Fulica atra, Linn.

Buff. Pl. enl. t. 179. - Im Winter in grossen Schaaren auf den Seen Unter-ëgyptens.

674. Fulica cristata, Linn.

Buff. Pl. enl. t. 797. Häufig auf dem Tana-See in Abyssinien.

\section{ORDNUNG. NATATORES (SCHWIMMVÖGEL).}

\section{A. ANATIDAE.}

1. Phönicopterinae.

675. Phönicopterus (Lin $\mathrm{n}$.$) roseus, \mathrm{P}$ all.

Ph. antiquorum, Temm. - Buff. Pl. enl. t. 63. - In grossen Flügen am Mittelmeer und seinen Brackwassern, einzeln auf der Nordhälfte des rothen Meeres und am Nil bis Ober-Ägypten.

676. Phönicopterus minor, Vi eill.

Vieill. Gal. t. 273. - In zahlreichen Schaaren auf den südlichen Theilen des rothen Meeres, einzeln am Bahr el abiad und am blauen Fluss.

*677. Phönicopterus erythraeus, B on a p. (?)

Häufig in den Syrten und ostwärts bis zum Cap Rasat.

\section{Cyg in a e.}

*678. Cygnus (Briss.) Olor, Linn.

Im Winter einzeln und in kleinen Flügen in Unter-Ägypten, vorzüglich bei Damiette.

*679. Cygnus musicus, Linn.

Wie der Vorhergehende. 
3. Plectropteriala

Alle Gänse heissen auf arabisch, "Wuss“ $\left(j_{y}\right)$.

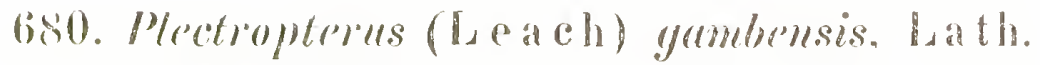

Mus. Senkenb. Vol. 3, t. 1. -- Mäufig am lana-See, an biatuen und weissen Fluss.

681. Sarkidionis (Eyton) melanonotos, Pennant.

Vieill. Gial. t. 28:. - Parweise und in grösseren Gesellschaflen in Schoa, am fanzen blauen und weissen Fluss und den Sümpfen tropdofans.

68\%. Chemaloprex (stephens) argyptiaca, Linn.

Buff. Pl. enl. t. 379. - Naun。 V. D. t. 294. - In ganz N. O. Afrika. llautierer an Nil als in Abyssinien, nieht am Mees.

4. Inseriate.

*68\%. Auser (Dopiss) allifroms, Pennant.

Aures erythropus, Linn. - Anser intermedius, Natum. V. D. t. 288. - - In Winter zahlreich an den unterïgyptischen Seen.

684. Berniclu (B.iss.) Brentr, I'al!.

Anes roporucta, Belon. - Naum. V. D. t. 292. - Im Winter in kleinen liesellschaften in Unter-Ågypten.

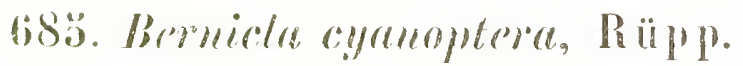

Ri ï p. Syst. Übers. t. 4\% - In Sehoa. Im Februar und Maip tral ieh sie nicht selten pataweise auf den Illochebenen von Woggara in Abyssinien an Wooren und Willbächen, und meist in Gesellschalt von Harpiprion carmiculata und Amas lencostigma.

5. A แ a $\mathrm{i}$ iा ae.

Dir Enten heissen ant aratiscel "Bal" (bs).

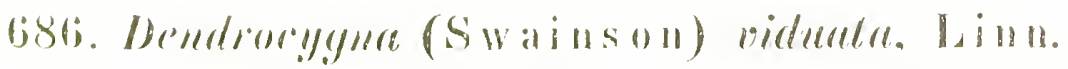

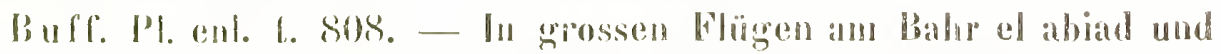
liahr el astak, ann Tana-See und den Sümpten Kordofins.

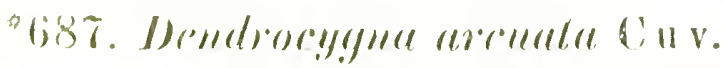

Häalig mil der Vorloregehenden in Kordofan.

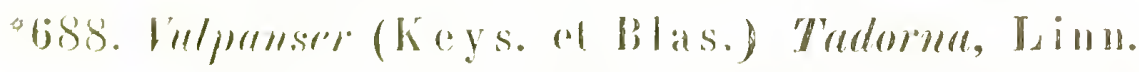

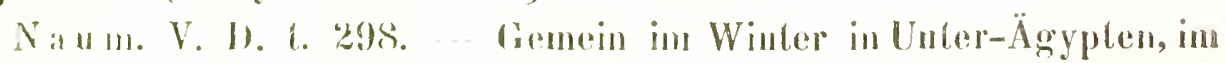

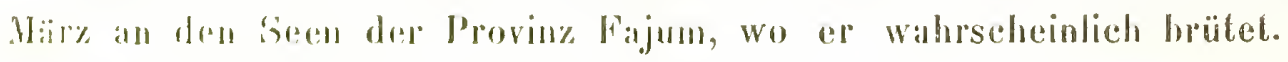

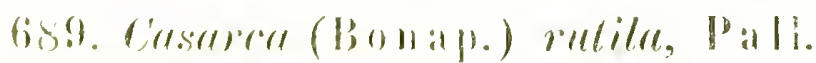

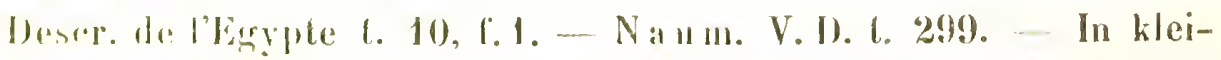

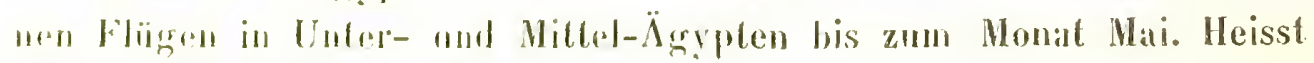

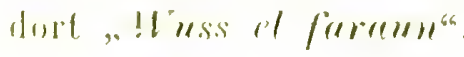


690. Poëcilonitta (Eyton) erythrorhyncha, Linm.

Smith, Ill. of South-Afr. t. 104. - Nach Rüppell hüufig in Sennaar und Abyssinien.

691. Mareca (Steph.) Penelope, Linn.

$\mathrm{Buff}$. Pl. enl. t. 825. - Naum. V. D. t. 305.- Im Winter in Ägypten häufig; nach Rüppell auch in Abyssinien.

692. Cyanopterus (Eyton) Querquedula, Linn.

Buff. Pl. enl. t. 946. - Naum. V. D. t. 303. - Häufig in N. 0. Afrika und Arabien.

*693. Chauliodus (Swainson) streperus, Linn.

Naum. V. D. t. 302. - Im Winter ziemlich häufig an den Nilmündungen und den benachbarten Seen.

694. Dafila (Leach) acuta, Linn.

Naum. V. D. t. 301. - In ganz N. O. Afrika südlich bis Kordofan und an dem weissen Fluss. Scheint fast hier zu brüten.

695. Anas Boschas, Linn.

Buff. Pl. enl. t. 776. - N a um. V. D. t. 300. - Im Winter in Unter-Ägypten, von Dr. R üpp ell auch in Abyssinien beobachtet.

696. Anas leucostigma, R ü $\mathrm{p} p$.

Anas sparsa, A. S mith, Ill. of South-Afr. t.97.-R üp p. Syst. Übers. t. 48. - Sehr gemein in Abyssinien; nicht westlich vom Tana-See.

*67. Anas flavirostris, Smith.

S mith, Ill. of South-Afr. t. 96. - In Abyssinien mit der Vorhergehenden.

698. Anas Crecca, Linn.

B u f. Pl. enl. t. 94\%. - N a um. V. D. t. 304. - Häufig an Nil und rothen Meer, auch am Tana-See in Abyssinien und den Sümpfen Kordofans beobachtet.

699. Rhynchaspis (Lea ch) clypeata, Li inn.

Anas clypeata, Linn. - Buff. Pl. enl. t. 971. - Na um. V. D. t. 306. - Hüufig in ganz N. 0. Afrika; brütet wahrscheinlich hier.

700. Oidemia (F lemming) fusca, Linn.

Buff. Pl. enl. t. 758. - Naum. V. D. t. 313. - Im Winter einzeln in Unter-Ägypten.

701. Undina (Keys. et Blas.) Mersa, Pall.

Descr. de l'Egypte t. 10, f. 2. - N a um. V. D. t. 315. - Im Winter in den Lagunen von Unter-Ägypten.

*702. Fuligula (Ray) Marila, Linn.

$\mathrm{N}$ a u m. V. D. t. 311. - Im Winter bis Mai in Unter-Ägypten u. Arabiell. 
503. Fuligulu cristutu, that y.

Ances fuligulr, Li inn. - Naum. V. D. t. 310. - Nach Dr. Rüppoll im Winter häufig in Abyssinien. Von mir blos in Unter-Ägypten einnesanmelt, wo sie den Winter uber gemein ist.

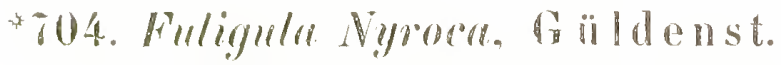

Naum. V. D. t. 309. - Anas lencophthalmos, beehst. - Nicht hä̈ntig in Winter in Unter- ̈̈rypten.

*alli. Fuliqula ferina, L inm.

$\mathrm{N}$ a u $\ldots$. V. I1. 1. 309. - Im Winter in Unter-Ägypten.

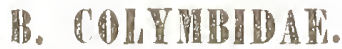

* Goti. Pordicepses (diatho) cristutus, Linn.

Nan m. V. D. t. 24t2. - Im Winter cinzeh! in Unter-Äsypten und III! Thuis.

* 807 . Podicepsesuberistutus, daceq.

$\mathrm{Naum.} \mathrm{V.} \mathrm{V.} \mathrm{1.} \mathrm{243.} \mathrm{-} \mathrm{In} \mathrm{Winter} \mathrm{einzeln} \mathrm{in} \mathrm{Unter-}$ in den Syrten angetroffen.

70s. Podiceps anritus, Bis iss.

$\mathrm{N}$ al $1 \mathrm{~m}$. V. D. 1. 246. - Im Winter in Unter-Ägypten und an Meerbusen von Sue\%.

7ols. Porlicepos minor, Hath.

Buff. Pl. enl. 1. 90:. Na um. V. D. 1. 947. Einzeln an derabyssi-

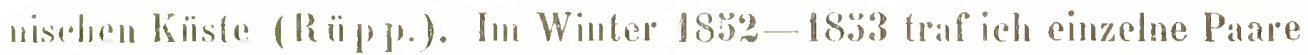

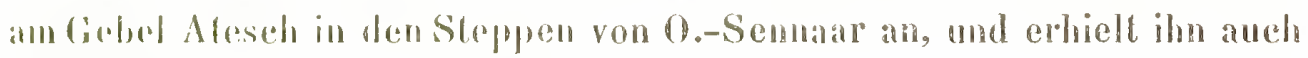

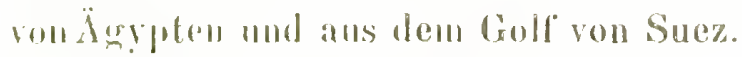

"ilu. colymbus septentrionulis, lainn.

Nanm. V. I. 1.

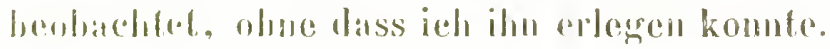

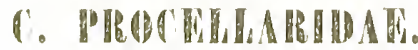

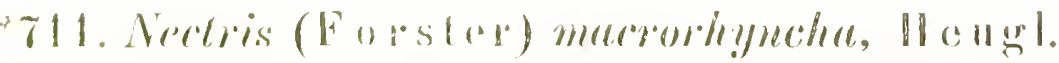

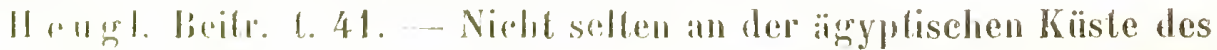

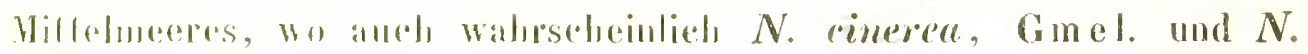

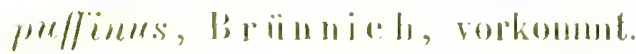

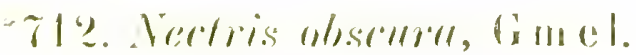

Sintlen and for ingplischen Noldküste, wo ich ein gestrandeles lixpouplater findl. 


\section{I A R I D A E.}

\section{Larinar.}

Die Möven heissen auf' arabisch „Nurseh" (jورئ).

713. Larus (Linn.) marinus, Gm.

B u f f. Pl. enl. t.266. - N a u m. V. D.t.268. - Einzeln an der Küste des mittelländischen Meeres das ganze Jahr hindurch.

714. Larus fuscus, Linn.

$\mathrm{N}$ a u m. V. D.t. 28\% - L. flavipes, M a y er et Wolf. - Häufig in Ägypten, sowohl am mittelländischen Meer als am Nil; kommt auch auf seinen Wanderungen bis auf den weissen und blauen Fluss.

715. Larus cachinnans, Pall.

Am rothen Neer und bei Damiette.

716. Larus argentatus, B rünnich.

B u f.Pl. enl.t. 253. -- Na u m. V. D. t. 266. - Sehr häufig am mittelländischen Mleer, einzeln längs des Nils bis Chartun.

*717. Larus canus, Linn.

Naum. V. D. t. 261. - Im Winter einzeln an den Küsten des Mittelmeeres.

718. Larus Ichthyaëtos, $\mathrm{P}$ all.

R üpp. Atl. t. 17. - Am rothen Meer, auf dem weissen Fluss und nach Dr. R ü p pell bei heftigem S. O. Wind im Frühjahr bei Cairo. Wahrscheinlich auch an den Küsten des Mittelmeeres.

719. Larus capistratus, T $\mathrm{mm}$.

Larus ridibundus, Lin n. (?) - B u ff. PI. enl. t. 970. - Das ganze Jahr hindurch in Unter-Ägypten.

720. Larus leucophthalmos, Licht.

T e m m. Pl. col. t. 366. - Am mittelländischen und rothen Meer.

721. Larus gelastes, Li cht.

L. leucocephalus, B o is s o n. - Im Frühjahr in Unter-Ägypten.

*722. Larus subroseus, He ugl.

H eug l. Beitr. t. 42, f. 1. - An den Küsten des rothen Meeres.

*23. Larus Brehmii, He ugl.

H eu g l. Beitr. t. 42, f. 2. - Am rothen Meer.

*724. Larus affinis, H eugl.

He u gl. Beitr. t. 42, f. 3. - Im Frühjahr in Ober-Ägypten beobachtet.

*725. Larus melanocephalus, T e $\mathrm{mm}$.

Naum. V. D. t. 259. - Im Winter und Frühjahr häufig bei Alexandria. 
*

$\mathrm{N}$ a um. V. D. t. ¿zos. - Im Winter und Fribjahr eben nicht selten an der Küste des Mittelmecres. Im Mai traf ich ihn ofters noch an, und zwal im schönsten Frihlingskleid, woraus ich schliesse, dass einzelne im Sommer gar nicht wegraehen.

\section{R h y f ha}

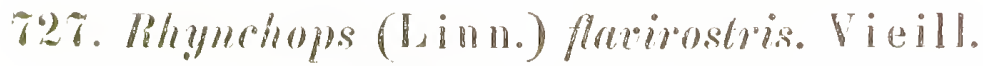

Pr. orimalis. Rüp p. Atl. t. 24. - In Familien und grossen Flïgen lïngs des ganzen Nils und auf dem blauen Fluss. Scheint sogar in Ägyten zu brüten. Im Spätherbst beginnt er zu wandern und sammelt sich dann zu ungeheuren Scharen.

\section{Sterninar.}

728. Sterma (Linn.) Crspin. Pall.

Deser. de l'Egypte t. 9, f. 1. - Natum. V. D. t. 248. - Häufig in Ärypten und Nubien.

729. Sterna Hinumerdo, Iinn.

Naum. V. D. t. 2:\%\%. - Paarweise an der ïgyptischen Nordküste in Winter und Frïljahr.

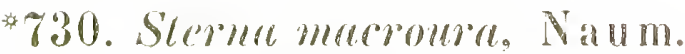

Sit. arelica, Temm. - Naum. V. D. t. 23:3. - Im Winter einzeln an der iingtischen Küste.

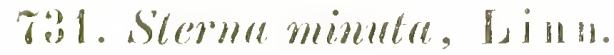

$N$ :1 11. V. D. 1. 2:31. - Im Winter und Frühjabr in Ägypten, sowohl :111 Heer als längs des Nils und seiner Canäle.

* 79:2. Sterma cantivere, Gm.

$\mathrm{N}$ a 1 m. V. I1. t. 2:00.- Einzeln bei Damiette, Alexandrien und in doll Sylden.

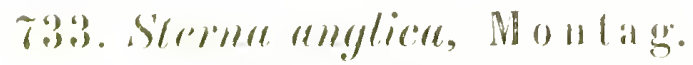

Jescr. de l'ligypte 1. 9, f. 2.-- Na u m. V. D. t. 249. - Häufig

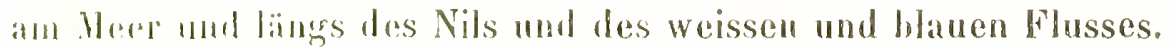

7:34. Sitrerme hyloridla, Pall.

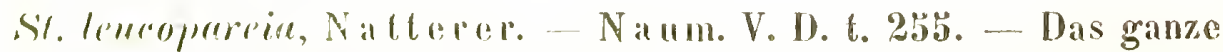
Jahr himdurch in İgghten und Nubien. Im Juli schoss ich äter junge Vongrol, die offenbar hice ansgrehrület worden waren.

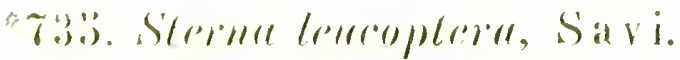

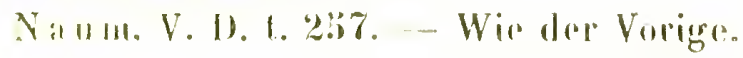


736. Sterna nilotica, Ha s s lqu.

Häufig in Ägypten und Nubien auf dem Nilstrom (R üp p.).

*737. Sterna naevia. Linn.

B u ff. Pl. enl. t. 924. - Längs dem Nil. (Mus, francof.)

738. Sterna nigra, Linn.

N aum. V. D. t. 256. - Nicht selten im Winter und Frühjahr an den Küsten des mittelländischen und arabischen Meeres.

739. Sterna velox, Rüpp.

R üp p. Atl. t. 13. - Hüufig auf dem rothen Meer, oft in grossen Flügen.

740. Sterna affinis, Rüpp.

R üpp. Atl. t. 14. - Wie die Vorhergehende.

741. Sterna (Thalassipora, B o j e) infuscata, Li ch t.

Nicht selten am rothen Meer.

742. Anous (L e a ch) tenuirostris, T e $\mathrm{mm}$.

T e m m. Pl. col. t. 202. — An den Küsten und Inseln des rothen Meeres.

\section{R. PELLCANIDAE.}

743. Plotus (Linn.) Levaillantii, Te mm.

T e mm. Pl. col. t. 380. - Einzeln auf dem Tana-See und dem Bahr el asrak und abiad.

744. Phaëton (Linn.) phönicurus, Gmel.

Buff.PI. enl. t.979. - Auf den wärmeren Theilen des rothen Meeres.

745. Dysporus (Illig.) brasiliensis, Spix (?)

B uff. Pl. enl. t. 973. - (Wohl eine noch unbeschriebene Species.) Häufig auf dem rothen Meer.

746. Pelecanus Onocrotalus, Linn.

$\mathrm{N}$ a u m. V. D. t. 282. - In Ägypten.

747. Pelecanus crispus, Bruch.

Anm. Die Pelikane heissen auf arabisch "Gémel el Bahr" (حب) oder „Abu Schilba“ (ب.إ).

Brand t, Icon. animal. rossic. Aves t. 6. - Häufig in Ägypten und Nubien, ist aber wohl verschieden vom europäischen crispus.

748. Pelecanus rufescens, $\mathrm{L}$ ath.

Rüpp. Atl. t. 21. - Häufig in Nubien, Sennaar und am weissen Fluss, wie auch auf der Südhälfte des rothen Meeres. 
749. Pelecrmms minor, Rü p p.

R ïpp. Sỵst. Übers. t. 49. - Häufig in Unter-Ägypten (R ü p p.).

* 780). Pelecermus megalophus. He $n \mathrm{~g} \mathrm{l}$.

I. mitratus, Li cht. Abh. der Berliner Akademic, Jahrg. 1838, 1. 3, f. 2 (?) - Auf dem Bahr el abiad, südlich vom $8^{0} \mathrm{~N}$. B., einzein und in kleinen Gesellschaften.

öl. Phalarocorrax (Briss.) africame, Gmel.

Deser. de l'Exyte. t. 8, f. 2. - Häufig am Nil, am weissen und hlauen Fluss und in Alyssinien. Geht nördlich bis zur Meeresküste.

ร:2. Phalucrocorax pygmaens, $\mathrm{P}$ all.

N a um. V. D. t. 281. - Von mir blos in Unter-Ägypten im Winter und Früligahr angetroffen; nach Riuppell auch in Abyssinien.

75:3. Phalacrocorax Carbo, Linm.

B uff. Pl. enl. t. 92\% - N a um. V. D. t. 279. - Häufig im Winter und Frühjahr im Delta und in Ober-Ägypten (Gebel Teer), auch an der arabischen Küste des rothen Meeres.

7o 4. Phalacrocorar lugubris, R ï p p.

Rüpp. Syst. Übers. t. 30. - Nach Rä ïppell häufig in Abyssinien. Ist -.. wie es scheint - von mir dort iibersehen worden. 


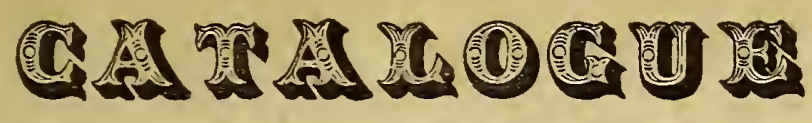

DE LA

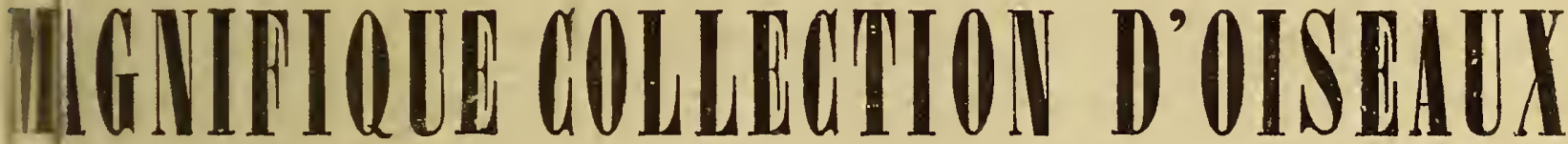

\section{DE M. LE PRINCE D'ESSLING, DUC DE RIVOLI,}

DONT LA VENTE AURA LIEU AUX ENGHERRS PUBLIQUES

DANS SA GALERIE, RUE DE LILLE, 98,

\section{le 8 Jovin 1846}

2 JosQuá $2 \mathrm{a}^{3}$ DU MÊME MOIS ET JOURS SUIVANTS, S'IL Y A LIEU,

URAL HISTO

A MIDI PIÉCIS,

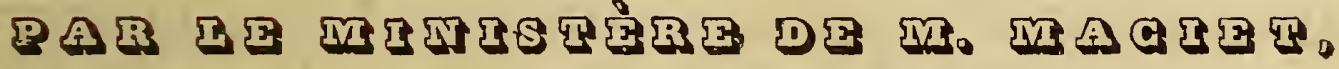
COMIIISSAIRE-PRISEUR A PARIS, CITÉ TRÉVISE, 10.

Ezy sition publique, du dimanche 31 mai jusqu'au 7 juin, de midi à 4 heures. ACQUÉREURS PAYERONT, EN SUS DU PRIX D'ADJUdication, 5 GENT. PAR FRANG, APPLICABLES AUX FRAIS DE VENTE.

Le italogue se distribue chez MM. Canivet, rue Saint-Thomas-du-Louvre, 24 ; et Parzudari, n: du Bouloi, 2, qui se chargeront des commissions des personnes qui ne pourraient pas a ister à la vente.

S'ADRESSER, POUR RENSEIGNEMENTS PRÉALABLES, A M. CANIVET.
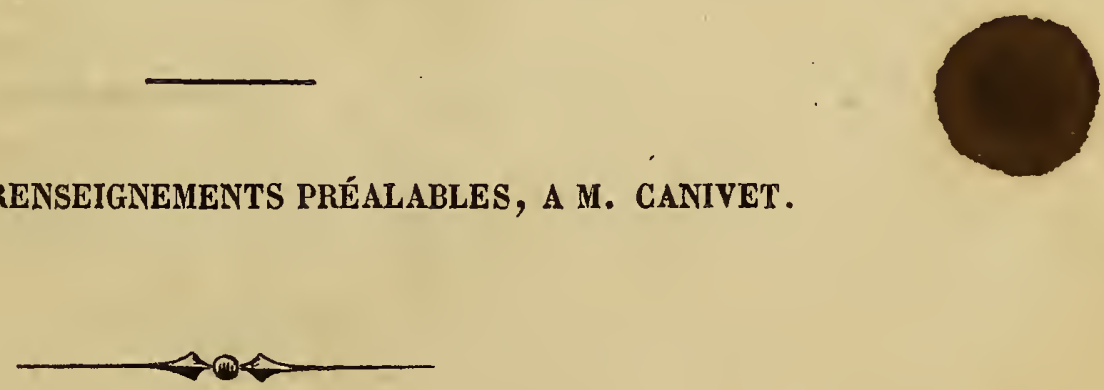

PARIS,

IMPRIMERIE SCHNEIDER ET LANGRAND, RUE D'ERFURTh, 1. 
Lia collection du prince l'Essling, dont nous publions ici le catalogue, est tre omme pour que nous entrions dans des détails circonstanciés sur sa composition nuns dirons seulement que c’est la plus belle qui ait jamais été mise en rente, "qu'elle renferme, a peu de chose près, toutes les espèces connues jusqu’à ce jout lons les individus sont de la plus grande fraitheup, et nous ne signalerons pas 1 wipices les plus rares qui s'y trouvent, parce que le nombre en serait trop consid. able, et que les amateurs les reconnaitront facilement en parcourant le catalogu "yiri présente un total de près de 4,000 capreces et de 10,000 exemplaires. Presqu foujours on trouvera réunis les deux sexes el les divers àges de chaque espèce lor qu'il prísentent des différences de phumage.

Nous derons farre observer que, pour faciliter les recherches, nous avons, da thacmo des grandes divisions de la classe des oiscaux, placéles genres dans l'ord Aphabilique, et que nous arons suivi le même systime pour les espèces de chaqu senre.

A lia fin de la dernière vacation, il sera vendu gra armoires ayant a mètres 11 cen Ho hantenr, I mitre 5.4 cent. de largeur et 62 cent. de profondeur; chaque armoi wh firmíc par une porte à deux battants, à montants et traverses on fer; les battan -unt garnis de 3 vitres de 85 centim. sur 32 centim.
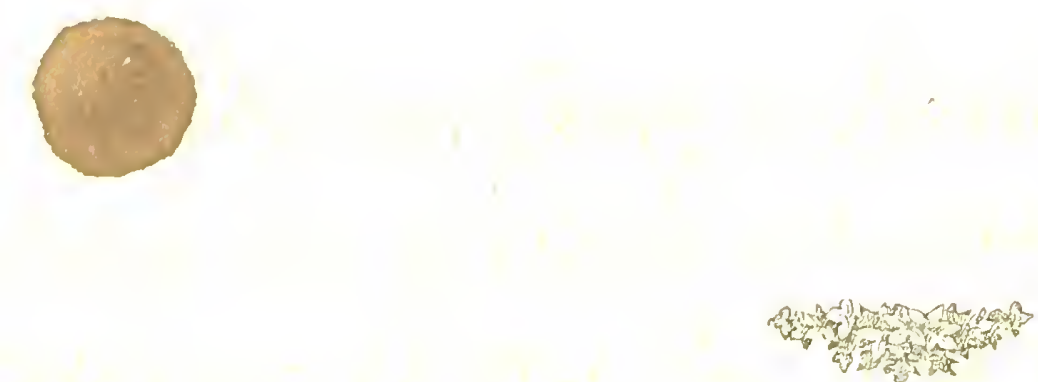


\section{CATALOGUE DES OISEAUX}

DE LA

COLLEGTION DE' M. LE P. D'E $\mathbf{E}^{\star \star \star \star * *}$.

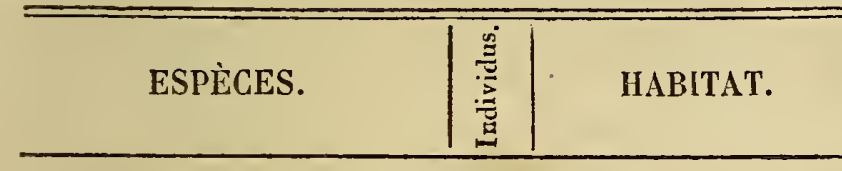

ACGIPITRES.

Genre catgartis.

Aura.

I 2 |Amérique.

Genge ratco.

Esalon.

Aguia.

Albicilla.

Albo-gularis.

Apivorus.

Aquilinus.

Aler.

Aterrimus.

Alricapillus.

Augur.

Aurantius.

$1=$ Aviceda

Bacha.

Banksii.

Bellicosus.

Berigora.

Biarmichus.

Bidentatus.

Bonelli.

Borealis.

Brachydactylus.

Brachypterus.

Brachyurus.

Brasiliensis.

Busarellus.

Bulco.
6 Europe.

9 Amérique mér.

6 Europe.

1 Mexique.

10 Europe.

3 Amérique mér.

5 Europe.

1 Amérique mér.

1 Cayenne.

3 Abyssinie.

5 Amérique mér.

3 Afrique.

8 Afriq. et Japon.

1 Inde.

2 Afrique.

7 Nouv.-Hollande.

7 Afrique.

9 Amérique mér.

6 Europe.

4 Amérique sept.

2 Europe.

6 Amérique mér.

1

5 Amérique mér.

4 Cayenne.

13 Europe.

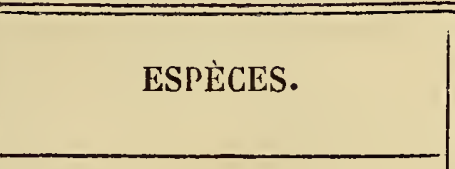

Buzon.

Cachinnans.

Carulescens.

Calei.

Capensis.

Cayennensis.

Cenchroïdes.

Chicquera.

Chimango.

Cinerascens.

Columbarius.

Concentricus.

Concolor.

Coronatus (Azara).

Coronalus.

Crislatellus.

Cuculoides.

Cuvieri

Cyaneus.

Degener.

Deiroleucus.

Destruclor.

Diodon.

Dussumieri.

Ecaudalus.

Eleonora.

Erythronolus.

Exilis.

Femoralis.

Frontalus.

Fulvus.

Furcalus.

Fuscosus.

Fuscus.

Gabar.
HABI'TAT.

Cayenne

3 Amérique mér.

Moluques.

2 Nouv.-Hollande.

Afrique.

2 Cayenne.

4 Nouv.-Hollande.

7 Inde.

2 Amérique mér.

15 Europe.

2 Amérique sept.

6 Amérique mér.

2 Afrique.

4 Amérique mẻr.

1 Afrique.

2 Inde.

4 Java.

2 Afrique.

6 Europe.

6 Amérique mér.

2 Ibid.

2 Ibid.

Ibid.

8 Inde.

6

1 Europe.

5 Amérique mér.

7 Afrique.

$\Xi$ Amérique mér.

1 Nouv.-Hollande.

6 Europe.

2 Amérique sept.

2 Nouv.-Hollande.

10 Inde.

8 Afrique. 


\begin{tabular}{|c|c|c|c|c|c|}
\hline ESPECES. & 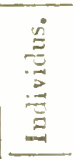 & MABITAT. & ESPECES. & 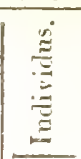 & HABITAT. \\
\hline Girommieri. & 1 & philippines. & Nisoides. & 4 & Brésil. \\
\hline Guianensis. & 1 & Guiane. & Nisus. & 15 & Europe. \\
\hline Gymnogenys. & 3 & Madagascar. & Nilidus. & 8 & Amérique mér. \\
\hline Halictus. & 8 & Europe, ete. & Nivars. & 2 & Java. \\
\hline Hamatus. & 8 & Amérique mér. & Nolatus. & 5 & Nonv.-Hollande. \\
\hline Harlami. & 1 & Amérique sept. & Novo-Hollandice. & 4 & Ibid. \\
\hline Hemidactylues. & 8 & Amérique mér. & Nover-Zclandice. & 1 & Nouv.-Zélande. \\
\hline Histrionicus. & 8 & loid. & Occipilalis. & 5 & Afrique. \\
\hline Tchthintus. & Bi & Inde. & Ocernicus. & 1 & Célébes. \\
\hline Imperialis. &. & Europe. & Ornalus. & $\ddot{z}$ & Amérique més. \\
\hline Fslundicus. & 4 & Ibid. & Palliatus. & 4 & Ibid. \\
\hline Jackal. & 8 & Afripue. & Pallidus. & 8 & Europe, elc. \\
\hline Japmicus. & 1 & Jap̣nn. & Palumbarius. & $\dddot{g}$ & Europe. \\
\hline Kieneri. & 1 & Ilimalaya. & Palusiris. & 8 & Brésil. \\
\hline Laccrmulatus. & 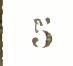 & Amérique mér. & Darasilicus. & 4 & Afrique. \\
\hline Lagopus. & $\ddot{3}$ & Europe, elc. & Pennalus. & 5 & Europe. \\
\hline Eencanchen. & $\ddot{3}$ & Brésil. & Pensyluanicus. & 6 & Amérique sept. \\
\hline leucocephalus. & 1 & Europe. & Peregrinus. & 鼻 & Eur, , Ar.,Ame̊r. \\
\hline Lencogaster. & 5 & Alrique. & Phalcobanus. & 2 & Amérique mér. \\
\hline Leucorrhous. & 2 & Brésil. & Plumbers. & 7 & Amérique. \\
\hline Toucosternus. & 2 & Nouv.-llollande. & Poecilonotos. & 4 & Cayenne. \\
\hline Limmetus. & 3 & Inde. & Polingaster. & 1 & Brésil. \\
\hline lineatus. & 4 & Anérique sept. & Poliogenys. & 4 & Ile de Lucon. \\
\hline Linnei. & f & $\operatorname{lng}$ & Polyzonoides。 & 2 & Afrique. \\
\hline Eiventer. & 7 & Inde. & Polyzonus. & $\ddot{3}$ & Amérique mér. \\
\hline Iopplotes. & 2 & Ibid. & Ponticerianus. & 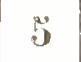 & Inde. \\
\hline Marei. & 3 & Iloid. & Prerocles. & 4 & Brésil. \\
\hline Mardagascarionsis. & 3 & Madägascar. & Plilorhynchus. & 1 & Java. \\
\hline Magnirostris. & $1 \%$ & Ambrique mir. & Punclulus. & 2 & Inde. \\
\hline Malayensis. & 1 & Simmatra. & Radialus. & $\ddot{3}$ & Nouv--Ilollande. \\
\hline Mnuduygli. & 1 & Amsique mer. & Prunivorus. & 7 & Mfrique. \\
\hline Mumius. & 8 & Mripne. & Rapax. & $y$ & Ibid. \\
\hline Molnuselencus. & 2 & lude. & Rengalis. & 5 & Amérique sept. \\
\hline Mrtineys. & 2 & Ambripne mer. & Beinumerdlie. & 1 & Inde. \\
\hline Helamonterus. & $\ddot{3}$ & Mriphos. & Rioromrie. & 1 & Aripue. \\
\hline Melomolis. & 1 & firpoir. & Paufifrons. & 5 & Amérique mér. \\
\hline mileus. & 2 & Europe. & Dhafipes. & 7 & Europe. \\
\hline Minimus. & 1 & Calyeme. & Rufiventris. & 自 & Mrique, cte. \\
\hline Hinulles. & ! & Mrivgur: & Raufies. & 11 & Hurope. \\
\hline Moluecensis. & 1 & Holnymes. & Irupicolovides. & 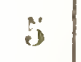 & Afrique. \\
\hline Monogfammicus. & 2 & Sémigrill. & Rupicolus. & 3 & libia. \\
\hline Mullizonus. & 1 & & Rulilans. & 9 & Amerrique mér. \\
\hline Musicus. & 12 & Mrique. & Sancli-Jollannis. & 1 & Amériqure sept. \\
\hline Fimorus. & $\because$ & Findepe. & Sparverins. & 26 & Am. sepit., ele. \\
\hline liger & 2 & Sincigal. & Sphenurus. & 5 & Nouv.-Ilollande. \\
\hline
\end{tabular}




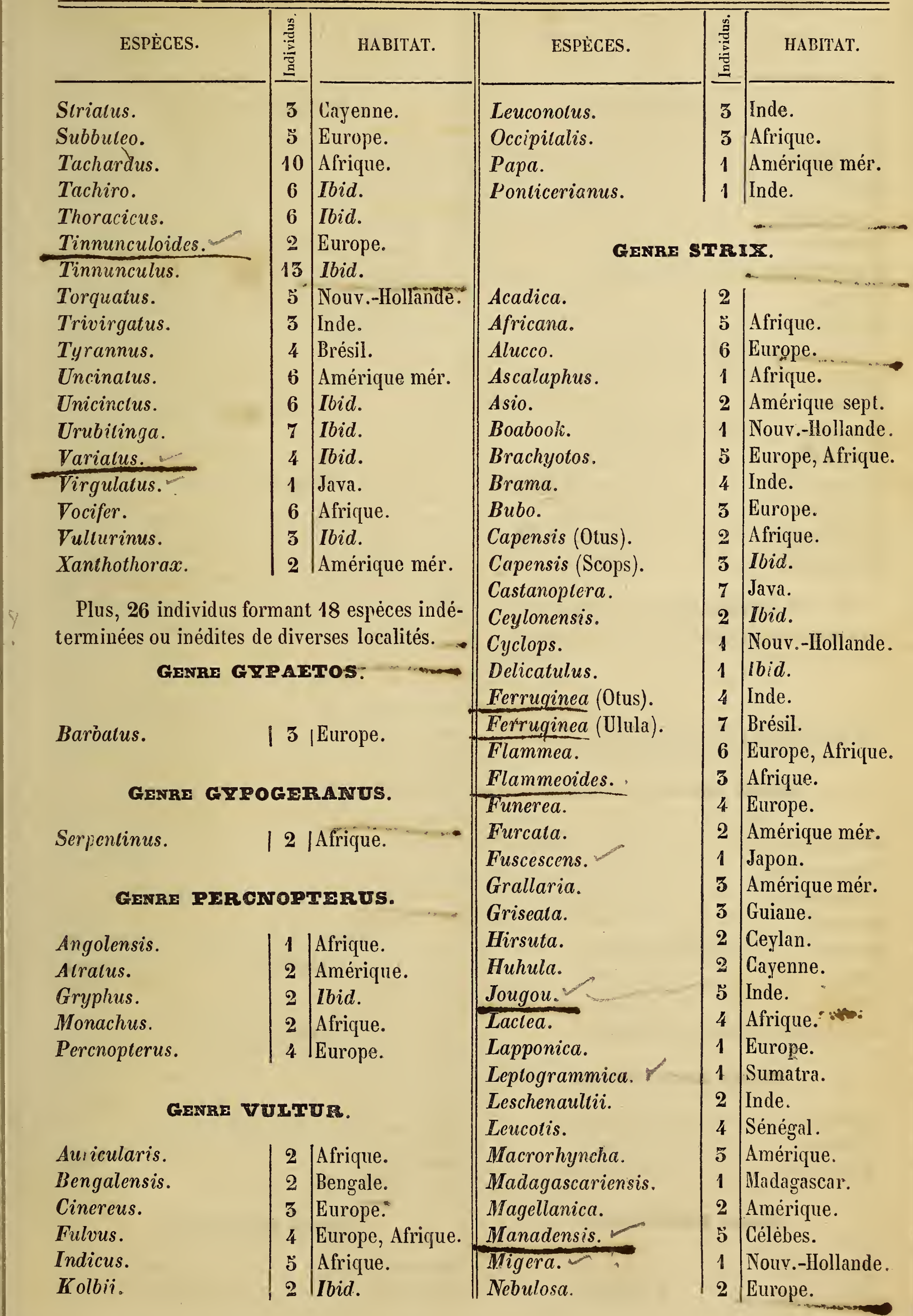




\begin{tabular}{|c|c|c|}
\hline ESPËCES. & 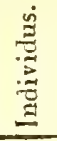 & HABITAT. \\
\hline Noctua. & 4 & Europe. \\
\hline Nyclea. & 2 & Ibid. \\
\hline Occipilalis. & 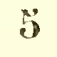 & Afrique. \\
\hline Otus. & $\breve{~}$ & Enrope \\
\hline Pagodarum. & 2 & Inde. \\
\hline Passerina. & 1 & Europe. \\
\hline Passerinoides. & 4 & Brésil. \\
\hline Perlata. & 1 & Sénégal. \\
\hline Personata. & 4 & Nouv.-Hollande. \\
\hline Pumila. & 2 & Amérique mér. \\
\hline Scops. & $\%$ & Europe, Afrique. \\
\hline Strenua. & 2 & Nouv.-Hollande. \\
\hline Strepilans. & 1 & Japon. \\
\hline T'engmalmi & 2 & Europe. \\
\hline Torquala. & 4 & Amérique mér. \\
\hline Uralensis. & 1 & Europe. \\
\hline Virginiana. & 2 & Amèr. du Nord. \\
\hline Woodfordi. & 1 & Afrique. \\
\hline
\end{tabular}

Plus, 55 individus formant 50 especes indéterminées on inédites de diverses localités.

\section{PASSEREAUX DENTIROSTRES.}

\section{GENRE ACARTHER.}

\section{Albifrons.}

Chrysorrheece

Diemensis.

Frontalis.

Lineata.

Nana.

Pusjlla.

reguloides.

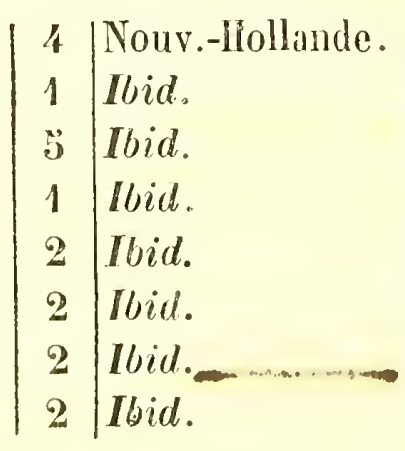

GENRE $\triangle$ CCENTOR.
1/pimus.
Calliope.
Mordularis

GeNhe ALIOTRIUS.

Carulea.

Carnifex.

Cayana.

Colinga.

Cucullata.

Lamellipennis.

Maynama.

Pompadoura.

Purpurea.

Pubrocristala.

Spixio.

Ventralis.

\section{GENRE AIMEIIS.}

\section{HABITAT.}

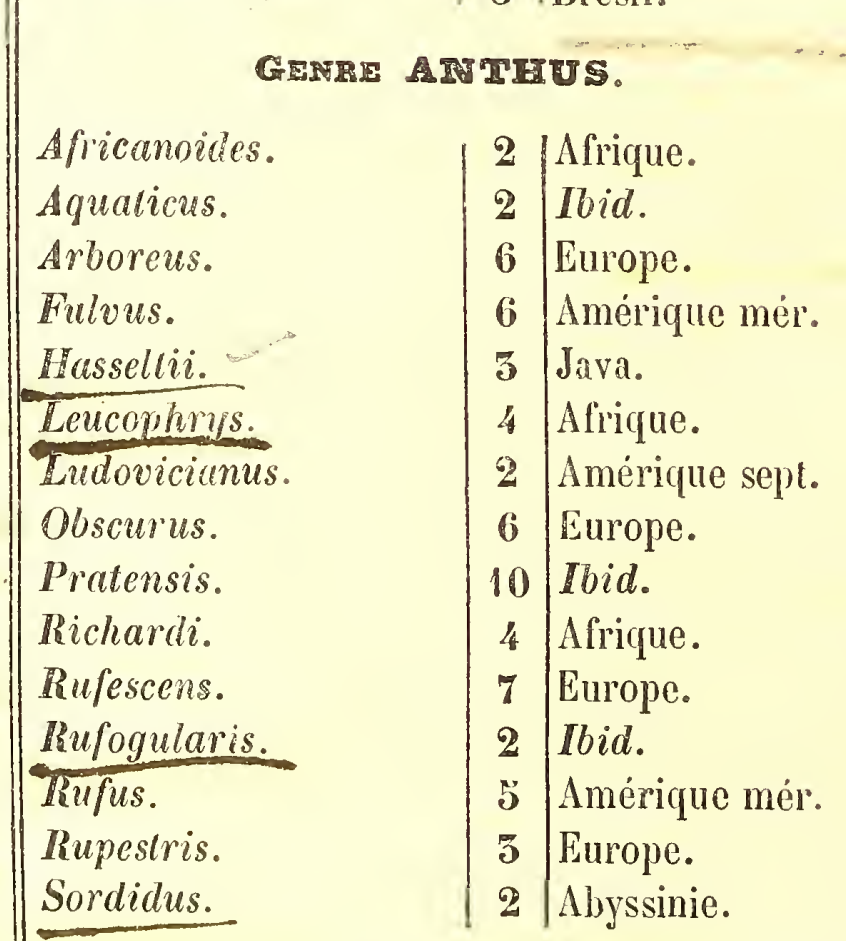

Plus, 28 individus formant 14 espèces indéterminées ou inédites provenant de diverses localités.

\section{GERRE BARITA.}

Anaphonesis.

Chalibara.

Sirepera.

\begin{tabular}{l|l|l} 
Iibicen. & 6 \\
Espece nouvelle. & Nouv.-Hollande.
\end{tabular}
GENRE BTEYIUS.

Melanoleucus.

picalus. \begin{tabular}{l|l}
5 & Nouv.-Illollande. \\
1 & Nouv.-Guinée. \\
3 & Océanie. \\
6 & Nouv.-Ilollande. \\
2 &
\end{tabular}

\begin{tabular}{l|l}
2 & Arrique. \\
5 & Brésil.
\end{tabular} 


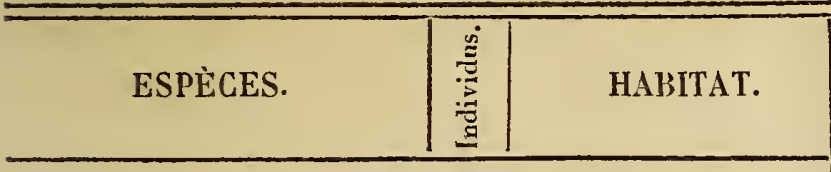

GENRE BOMBICILIA.

Cedrorum.

Garrula. $\mid$\begin{tabular}{l|l} 
こ & Amérique sept. \\
$\mathbf{2}$ & Europe.
\end{tabular}

GENRE CASMARHYNCEOS.

Carunculalus.

Nudicollis.

Variegata. $\mid$\begin{tabular}{l|l}
$\mathbf{3}$ & Cayenne. \\
5 & Brésil. \\
$\mathbf{1}$ & Ibid.
\end{tabular}

\section{GanRE CEBIEPHYRIS.}

\begin{tabular}{l|l|l|} 
Ater. & $\mathbf{2}$ & Afrique. \\
Cuna. & $\mathbf{2}$ & Nouv.-Guinée. \\
\hline Fimbriatus. & 4 & Java. \\
\hline Flavus. & $\mathbf{3}$ & Afrique. \\
Jalajalq. & 1 & Ile de Luçon. \\
Karu. & $\mathbf{3}$ & Nouv.-Hollande. \\
\hline Leucomelas. & 1 & Sumatra. \\
Lobalus. & 1 & Java. \\
Macei. & 1 & Ibid. \\
Madagascariensis. & 1 & Madagascar. \\
Melanoleucus. & $\mathbf{2}$ & Java. \\
\hline Mentalis. & 1 & Nouv.-Hollande. \\
Nova-Guinea. & 2 & Nouv.-Guinée. \\
Orientalis. & $\mathbf{3}$ & Inde. \\
\hline Papuensis. & $\mathbf{2}$ & Nouv.-Hollande. \\
Pecloralis. & 1 & Afrique. \\
Phonicoplerus. . & 2 & Ibid. \\
Tenuirostris. & $\mathbf{2}$ & Nouv.-Hollande. \\
Temminkii. & $\mathbf{1}$ & Célèbes. \\
Viridis. & $\mathbf{3}$ & Timor. \\
\hline
\end{tabular}

Plus, 23 individus formant 19 espéces indéterminées ou inédites provenant de diverses localités.

GENRE CEPHATOPTERUS.

Ornatus. | 2 |Amérique mér.

GENRE CaAMJiza.

Meruloides。

17 |Brésil.
ESPÈCES.

HABITAT.
Erythrocephala.

Punctala.

Ruficollis.

Variegata. $\mid$\begin{tabular}{l|l}
1 & Himalaya. \\
$\mathbf{3}$ & Nouv.-Hollande. \\
1 & Himalaya. \\
$\mathbf{2}$ & Ibid.
\end{tabular}

Plus, 2 espéces indéterminées ou inédites d'Himalaya.

\section{GENRE CINCLORA IIPHUS.}

Cruralis.

|. 1 |Nouv.-Hollande.

\section{GENRE CINCLUS.}

Aquaticus.

Melanogaster.

Pallasii.

Espéce nouvelle.

$\mid$\begin{tabular}{l|l}
3 & Europe. \\
1 & Ibid. \\
1 & Ibid. \\
1 & Ilimalaya.
\end{tabular}

\section{GENRE COLLURICINCLA.}

Cinerea.

Rectirostris.

Rufiventris.

2 Nouv.-Hollande. \begin{tabular}{l|l}
2 & Ibid. \\
1 & Ibid.
\end{tabular}

\section{GENRE CONIROSTRUM.}

\begin{tabular}{l|l|l|} 
Albifrons. & 2 & Bogota. \\
Caruleifrons. & 2 & Ibid. \\
Cinereum. & 1 & Pérou. \\
Rufum. & 3 & Bogota. \\
\hline Septicolor. & 2 & Ibid. \\
\hline
\end{tabular}

\section{GenRe CORACIISA.}

Sculala.

14 |Brésil.

\section{GenRe cossy pha.}

Humeralis.

12 |Afrique.

\section{GENRE CRATEROPUS.}

Bicolor.

Jardinii.

Leucocephalus.

Leucopygius.

Espèce indéterminée.

\begin{tabular}{|l|l}
3 & Afrique. \\
4 & Ibid. \\
4 & Ibid. \\
2 & Ibid. \\
1 &
\end{tabular}




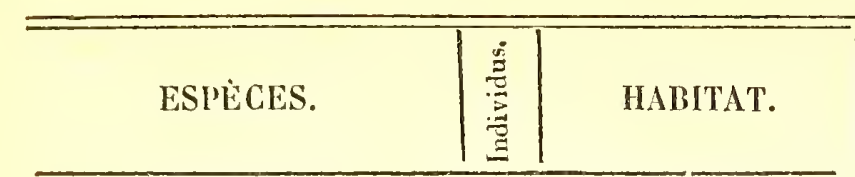

\section{GenRe cROcins.}

Gullalus.

12 Java.

\section{GenRE DICRURUS.}

Aneus.

Balicassius.

Forficalus.

Hotlenlolus.

Leucophaus.

Macrocercus.

Malabaricus.

Minulus.

Musicus.

Remifer.

Plus, 6 individus formant 4 espèces indéterminées ou inédites provenant de diverses localités.

\section{GENRE DREMOPHIEA.}

Carinala.

Velala.

$\mid$\begin{tabular}{l|l}
2 & Nouv.-Hollande. \\
6 & Bornéo.
\end{tabular}

GENRE RDOIIUS.

Puellus.

| 6 | Sumatra.

\section{GENRE ENICUITS.}

Coronalus.

Maculatus.

Ruficapillus.

Velatus.

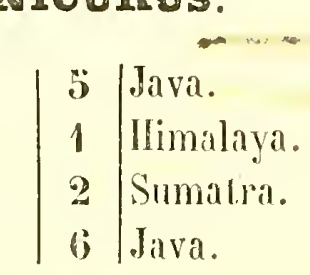

GENRE IEUPETTS.

Macrocercus.

| 2 | Sumatri.

GENRE RURYCEROS.

I'recoslii.

2 Madagascar.

GENRE EURYIAIITUS.

Corydon.

Cucullatus.

\begin{tabular}{l|l}
2 & Simmatra. \\
3 & Iloid.
\end{tabular}

\begin{tabular}{|c|c|c|}
\hline ESPECES. & 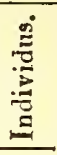 & HABITAT. \\
\hline Horsficldii. & 2 & Java. \\
\hline Javanicus. & 2 & Ibid. \\
\hline Lunulatus. & 1 & Ibid. \\
\hline Nasulus. & 2 & Ibid. \\
\hline Psillacinus. & 2 & Ibid. \\
\hline
\end{tabular}

GENRE FALCUNCUIUS.

Fronlatus.

Leucogaster. \begin{tabular}{l|l}
$\mathbf{3}$ & Nouv.-Hollande. \\
2 & Ibid.
\end{tabular}

GERRE CRACULA.

Musicus.

Religiosa.

\begin{tabular}{l|l}
2 & Inde. \\
3 & Ibid.
\end{tabular}

GENRE GRATIIRA.

Bicolor.

| 2 |Nouv -llollande.

GERE GYIIYOCPIIAEUS.

Capucinus.

1 3 lCayenne.

GEITRE GTITITODERA.

Nudicollis.

2 ICayenne.

\section{GENRE AIIPOTZTETS.}

Chrysorrhoa.

2 Mexicpue.

GENRE HIPSIPIETES.

Ganusa.

Olivacea.

\begin{tabular}{l|l|l} 
Ourovang. & Madagascar.
\end{tabular}

\section{GENRE ICTERTA.}

Auropectoralis. $|3|$ Amérique mér.

\begin{tabular}{l|l|l}
\hline Arcuala. & 2 & Bogota. \\
Viridis. & 5 & Ibid.
\end{tabular}

GENRE JORA.

\begin{tabular}{l|l|l} 
Quadricolor. & 4 & Inde. \\
Espece nouvelle. & 2 & Ibid.
\end{tabular} 


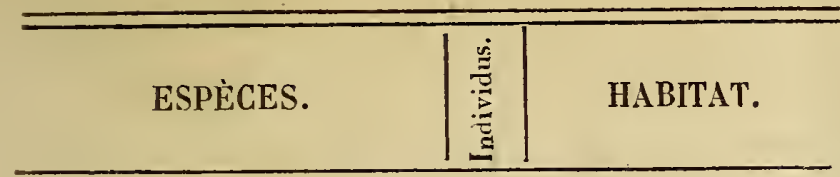

GENRE KITTA.

Albirostris.

Holosericea.

Sinensis.

Virescens.

\begin{tabular}{l|l}
2 & Afrique. \\
3 & Nouv.-Hollande. \\
4 & Chine. \\
1 & Ibid.
\end{tabular}

\section{GENRE IAMPROTORIIS.}

\begin{tabular}{|c|c|c|}
\hline Eneus. & 4 & Afrique. \\
\hline Albirostris. & 2 & Ibid. \\
\hline Auratus. & 3 & Ibid. \\
\hline Bicolor. & 2 & Ibid. \\
\hline Burchellii. & 2 & Ibid. \\
\hline Canlor. & 8 & Timor. \\
\hline Chalybous. & 2 & Afrique. \\
\hline Chrysonolis. & 2 & Ibid. \\
\hline Erythrophris. & 5 & Ibid. \\
\hline Leucogaster. & 4 & Ibid. \\
\hline Maurilianus. & 1 & Ibid. \\
\hline Melallicus. & 2 & Ibid. \\
\hline Morio. & 5 & Ibid. \\
\hline Nabirop. & 2 & Ibid. \\
\hline Nabouroup. & 2 & Ibid. \\
\hline Rufivenlris. & 6 & Ibid. \\
\hline Spilopterus. & 2 & Himalaya. \\
\hline Splendens. & 4 & Afrique. \\
\hline Superba. & 2 & Ibid. \\
\hline Splendidus. & 2 & Ibid. \\
\hline Teruirostris. & 3 & Ibid. \\
\hline
\end{tabular}

Plus, 8 individus formant $\mathbf{z}$ espèces indéterminées ou inédites provenant de diverses localités.

\section{GENRE IAINUUS.}

Athiopicus.

Albifrons.

Anguilimus.

Antiguanus.

Arcuatus.

Atrococcineus.

Australis.

Bacbakiri.

\begin{tabular}{|l|l|}
3 & Abyssinie. \\
2 & Amérique. \\
3 & Afrique. \\
1 & Amérique. \\
1 & Ibid. \\
2 & Ibid. \\
1 & Ibid. \\
5 & Afrique.
\end{tabular}

\begin{tabular}{|c|c|c|}
\hline ESPĖCES. & 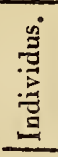 & HABITAT. \\
\hline Barbarus. & 2 & Afrique. \\
\hline Bicolor. & 1 & Madagascar. \\
\hline Canadensis. & 2 & Amérique sept. \\
\hline Capensis. & $\mathbf{3}$ & Afrique. \\
\hline Chlorocephalus. & 1 & Ibid. \\
\hline Collaris. & 4 & Ibid. \\
\hline Collurio. & 4 & Ibid. \\
\hline Corvinus. & 2 & Ibid. \\
\hline Crassiroslris. & 1 & Ibid. \\
\hline Cubla. & 4 & Ibid. \\
\hline Erylrhogaster. & 3 & lbid. \\
\hline Excubitor. & 5 & Europe. \\
\hline Guianensis. & 2 & Guiane. \\
\hline Gucturalis. & 3 & Afrique. \\
\hline Hardwickii. & $\mathbf{3}$ & Inde. \\
\hline Iclerus. & 2 & Afrique. \\
\hline Inornala. & 1 & Java. \\
\hline Lucionensis. & 2 & Japon. \\
\hline Ludovicianus. & 1 & Amérique sept. \\
\hline Meridionalis. & 1 & Europe. \\
\hline Minor. & 3 & Ibid. \\
\hline Olivaceus. & 3 & Afrique. \\
\hline Pyrrhonotos. & 2 & Inde. \\
\hline Sabinii. & 2 & Afrique. \\
\hline Senegalus. & 7 & lbid. \\
\hline Septentrionalis. & 1 & Amérique sept. \\
\hline Silens. & 3 & Afrique. \\
\hline Similis. & 3 & Ibid. \\
\hline Superciliaris. & 2 & Java. \\
\hline Striato-lhorax. & 2 & Amérique sept. \\
\hline Trifascialus. & 4 & Afrique. \\
\hline Virgatus. & 1 & Java. \\
\hline
\end{tabular}

Plus, 32 individus formant 19 espéces indéterminées ou inédites provenant de diverses localitès.

\section{GENRE ITPTONIX.}

Albicollis.

Macropus.

Rupecula.

$\mid$\begin{tabular}{l|l}
2 & Chili. \\
2 & Ibid. \\
1 & Ibid.
\end{tabular}

Plus, 2 individus formant 2 espèces indéterminées ou inédites du Ghili. 


\begin{tabular}{|c|c|c|}
\hline ESPËCES. & 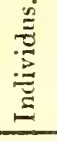 & HABITAT. \\
\hline \multicolumn{3}{|c|}{ GENRE MAIURUS. } \\
\hline Ácacio. & 1 & Afrique. \\
\hline Africanus. & 2 & Ibid. \\
\hline Browerii. & 2 & Nouv.-Ilollande. \\
\hline Castanea. & 5 & Afrique. \\
\hline Cyaneus. & 3 & Nouv.-IIollande. \\
\hline Elegans. & 1 & Ibid. \\
\hline Frenalus. & 5 & Amérique mél. \\
\hline Gracilis. & 1 & Afrique. \\
\hline Lamberti. & 2 & Nouv.-Hollande. \\
\hline Leucoplerus. & 2 & Ibid. \\
\hline Longicaudus. & 4 & Ibid. \\
\hline Macrourus. & 5 & Afrique. \\
\hline Malachurus. & 2 & Nouv.-Hollande. \\
\hline Marginalis. & 1 & Java. \\
\hline Melanocephalus. & 5 & Nouv.-Ilollande. \\
\hline Pectoralis. & 1 & Afrique. \\
\hline Phragmitoides. & 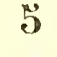 & Ibid. \\
\hline Polychrous. & 2 & Java. \\
\hline Ruficeps. & 1 & Nouv.-IIollande. \\
\hline Splendens. & 2 & Ibid. \\
\hline Textilis. & 1 & Ibid. \\
\hline Textrix. & 2 & Afriq̄ue. \\
\hline
\end{tabular}

Plus, 4 individus formant 2 espèces indéterminées ou inédites provenant de la NouvelleLélande.

\section{GENRE MANORHIRA.}

Melanocephala. Viridis.

$$
\mid \begin{array}{l|l}
5 & \text { Nouv.-IIollande. } \\
2 & \text { Ibid. }
\end{array}
$$

\section{GENRE MEITIPHAGA.}

\begin{tabular}{l|l|l|} 
Albifrons. & 1 & Nouv.-Hollande. \\
Argenticeps. & 2 & Ibid. \\
Alricapilla. & 4 & Ibid. \\
Auricomis. & 2 & Ibid. \\
Australasiana. & 5 & Ibid. \\
Carunculala. & 2 & Ibid. \\
Cincla. & 1 & Ibid. \\
Circinalus. & 2 & Ibid. \\
Citreogularis. & 4 & Ibid. \\
Corniculala. & $: 3$ & Ibid. \\
Cralieea. & 1 & Ibid.
\end{tabular}

\begin{tabular}{|c|c|c|}
\hline ESPÈCE. & 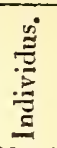 & HABITAT. \\
\hline Cyanotis. & 2 & Nouv.-Hollande. \\
\hline Dumerilii. & $\check{~}$ & Ibid. \\
\hline Fasciala. & 1 & Ibid. \\
\hline Flavigula. & 2 & Ibid. \\
\hline Fulvifrons. & 3 & Ibid. \\
\hline Gouldii. & 1 & Ibid. \\
\hline Gularis. & 1 & Ibid. \\
\hline Leucolis. & 2 & Ibid. \\
\hline Lewinii. & 1 & Ibid. \\
\hline Limbala. & 1 & Ibid. \\
\hline Lunulata. & 4 & Ibid. \\
\hline Maculata. & 1 & Ibid. \\
\hline Melanocephala. & 5 & Ibid. \\
\hline Mellivora. & 5 & Ibid. \\
\hline Nova-Hollandice. & 2 & Ibid. \\
\hline Obscura. & 1 & Ibid. \\
\hline Ocularis. & 1 & Ibid. \\
\hline Oriolides. & 2 & Ibidl. \\
\hline Ornala. & 1 & Ibid. \\
\hline Penicillala. & 1 & Ibid. \\
\hline Phrygia. & 3 & Ibid. \\
\hline Picla. & 1 & Ibid. \\
\hline Plectorhyncha. & 1 & Ibid. \\
\hline Reliculata. & $\mathbf{3}$ & Ibid. \\
\hline Rufogularis. & 2 & Ibid. \\
\hline Sericea. & 5 & Ibid. \\
\hline Sonora. & 5 & Ibid. \\
\hline Superciliosus. & 5 & Ibid. \\
\hline Unicolor. & 1 & Ibid. \\
\hline Validirostris. & 3 & Ibid. \\
\hline Viridis. & 2 & Ibid. \\
\hline
\end{tabular}

Plus, 10 individus formant 8 espèces indé. terminées ou inédites provenant de la Nouvelle-IIollande.

\section{GENRE EN URA.}

Novce-Hollandia. $\quad$ I 3 |Nouv.-Ilollande.

GENRE MEIRURAXIS.

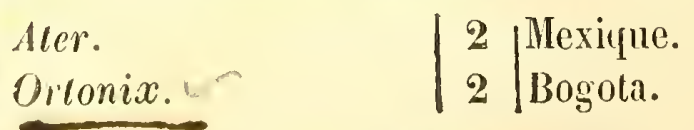

GENRE MIRTO.

Dumontii. 2 |Nouv.-Ginincé. 


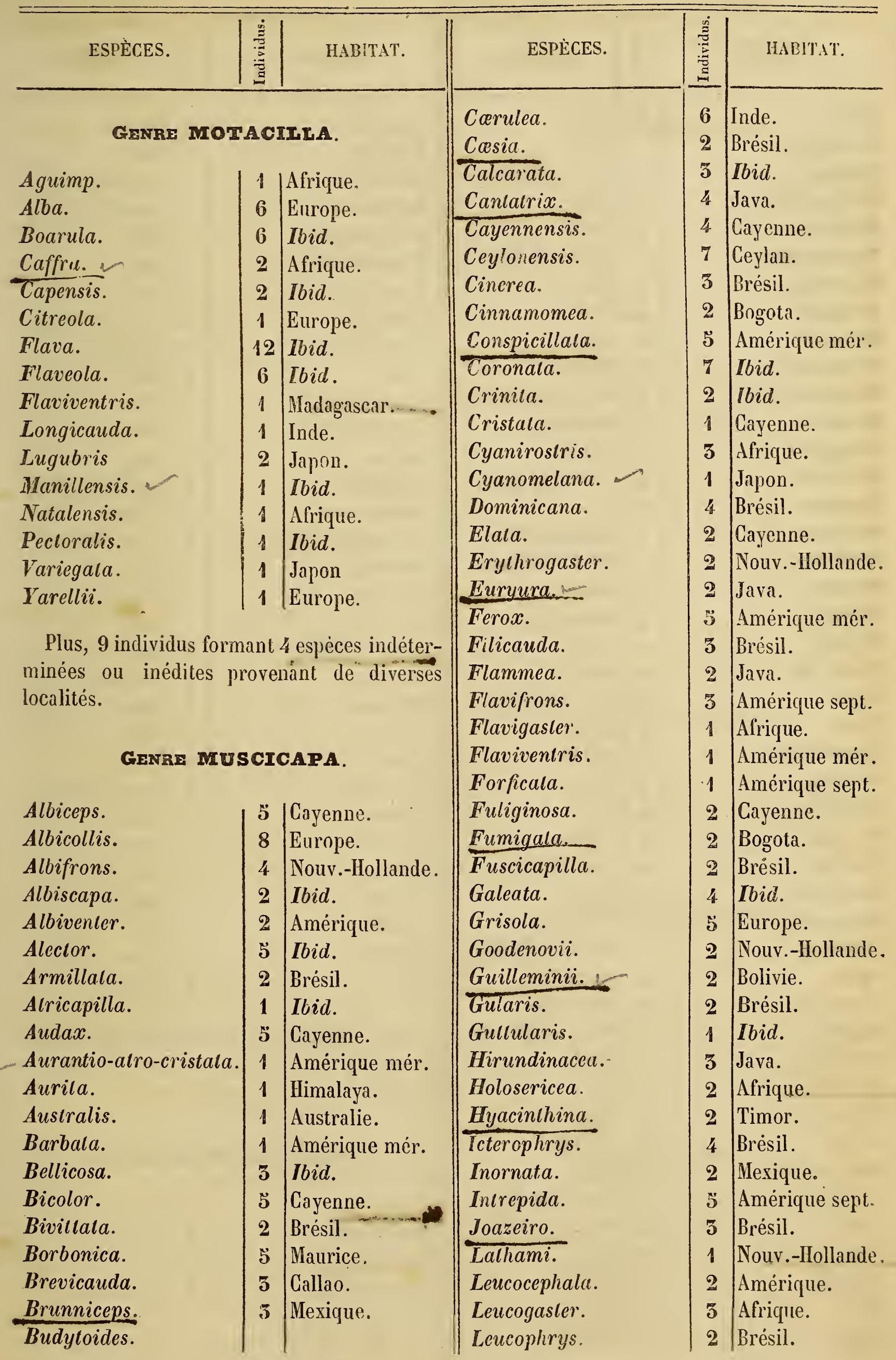




\begin{tabular}{|c|c|c|c|c|c|}
\hline ESPECES. & 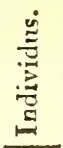 & HABITAT. & ESPËCES。 & 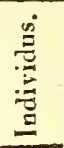 & HABITATุ. \\
\hline Lobata. & 巳̆ & Afrique. & Rufescens. & 2 & Bolivie. \\
\hline Longipennis. & 4 & Brésil. & Ruficeps。 & 1 & Brésil. \\
\hline Longipes. & 2 & Nouv.-Zélande. & Rufiventer. & 1 & Afrique. \\
\hline Luteocephala. & 4 & Brésil. & Rufiverlex. & 2 & Brésil. \\
\hline Maculata. & 2 & Ibid. & Rufogularis. & 2 & Ibid. \\
\hline Maculirostris. & 1 & Amérique mér. & Rufo-olivacea. & 2 & Ilbid. \\
\hline Martinica. & 5 & Ibid. & Rupestris. & 1 & Timor. \\
\hline Matulina. & 2 & Ibid. & Ruticilla. & 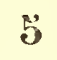 & Amérique sept. \\
\hline Melaleuca. & 2 & Ibid. & Scapularis. & 3 & Afrique. \\
\hline Melancholica. & 5 & Bolivie. & Scila. & 2 & Ibid. \\
\hline Melanocephala. & 5 & Amérique mér. & Semipartila. & 4 & ibid. \\
\hline Melanops. & 5 & Himalaya. & Senegalensis. & 音 & Ibid. \\
\hline Melanolis. & 3 & Brésil. & Stella. & 3 & Ibid. \\
\hline Mentalis. & ב & Amérique mér. & Straminea. & 2 & Amérique sept. \\
\hline Miniata. & $\breve{3}$ & Java. & Sulphurata. & 2 & Brésil. \\
\hline Monlana. & 1 & Amérique mér. & Telescophthalmus. & 1 & Nouv.Guinée. \\
\hline Multicalor. & 4 & Nouv.-IIollande. & Trichoma. & 1 & Java. \\
\hline Murina. & 2 & Bolivie. & Tricolor. & 2 & Timor. \\
\hline Mutala. & 2 & Madagascar. & Trivirgata. & 1 & Java. \\
\hline Mystax. & 1 & Brésil. & Tuberculifera. & 3 & Bolivie. \\
\hline Nigerrima. & $\ddot{3}$ & Bolivie. & Tyrannus. & 6 & Amérique sepi. \\
\hline Nilida. & 2 & Nouv.-Ilollande. & Variegalc. & 9 & Brésil. \\
\hline Niven. & 3 & Brésil. & Velssla. & $\mathbf{3}$ & Ibid. \\
\hline OEnanthoides. & 1 & Bogota. & Velox. & 1 & Java. \\
\hline Olivacea. & 9 & Brésil. & Verlicalis. & 2 & Amérique sepl. \\
\hline Omnicolor. & 3 & Chili. & Viduata. & 1 & Inde. \\
\hline Paradisi. & 8 & Ceylan. & Vieillotie. & 1 & Bolivie. \\
\hline Parulus. & 4 & Amérique mér. & Virens. & 5 & Brésil. \\
\hline Peregrina. & 3 & Inde. & Virgala. & ๖ & Bolivie. \\
\hline Philomela. & 1 & Afrique. & Viridis. & 2 & Brésil. \\
\hline Phonicea. & 1 & Nouv.-llollande. & Volitans. & 3 & Jav \\
\hline P'ipiri. & 2 & Orénoque. & Xeiapa. & 2 & Brésil. \\
\hline
\end{tabular}

Pitangua.

I'lumber.

Polygiotla.

Pomarea.

I'rinceps.

Priril.

Pristrinaria.

I'salura.

Pyrope.

$\frac{\text { Pyrrhoptera. }}{\text { Ouerula. }}$

Regia.

Rufa.

Amérique.

Nouv.-IIollande.

Brésil.

9 Taiti.

3 Ilimaliya.

4 Afrique.

3 Ilid.

2 Amérique sept.

:i Amirique mór.

2 Inde.

1 Bresil.

3 Ibid.

1 Bolivie.
Plus, 170 individus formant 92 espèces indéterminées ou inédites provenant de diverses localités.

\section{GenRe mophonos.}

\begin{tabular}{l|l|l} 
Glaucinus. & 5 & Java. \\
Trorsfieldit. & 1 & Ilimalaya. \\
Melallicus. & 5 & Java. \\
Temminkii. & 2 & Ilimalaya.
\end{tabular}




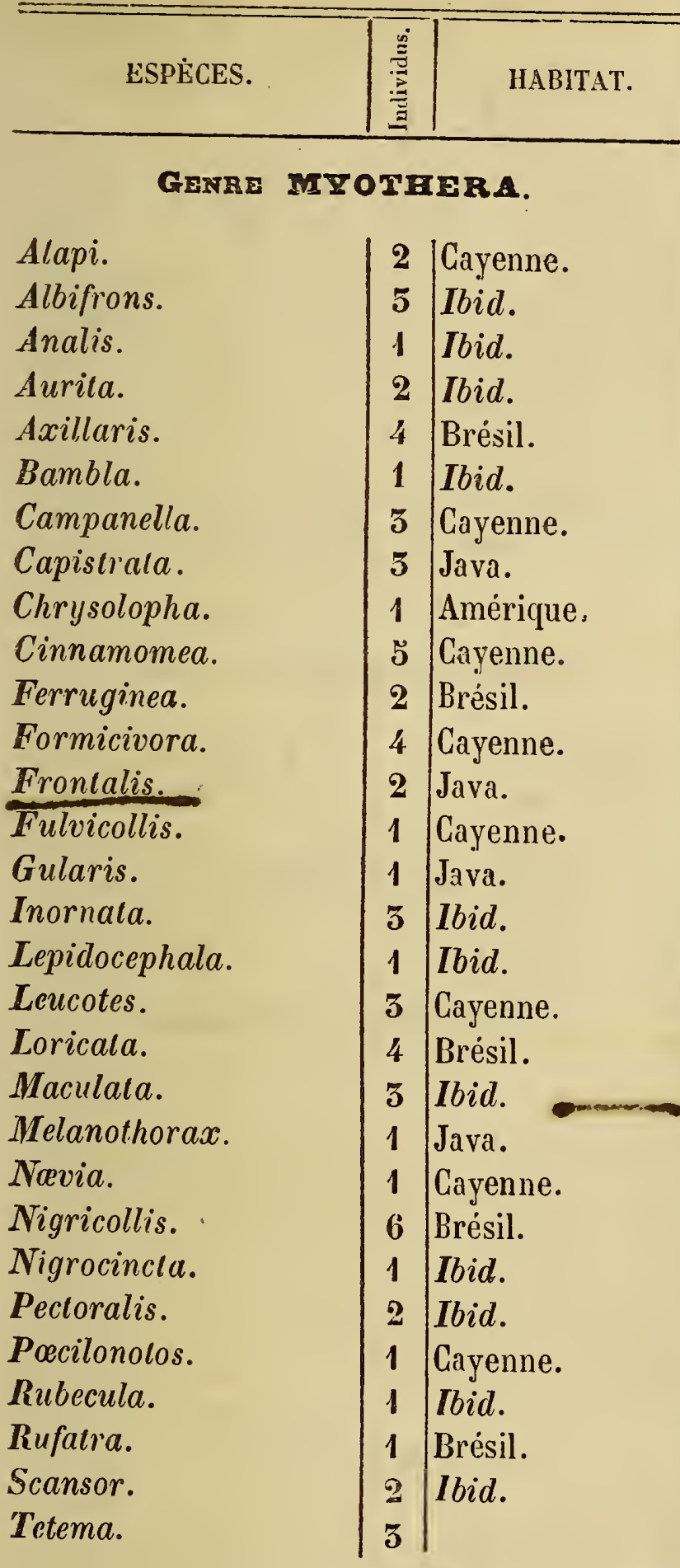

Plus, 16 individus formant 9 espèces indéterminées ou inédites provenant de diverses localités.

\section{GERRE OCYPTIRUS.}

Alboviltalus.

Fuscalus.

Leucorhynchos.

Personatus.

Rufiventer.

Sanguinolenlus.

Superciliosus.

Viridis.
4 Nouv.-Hollande.

1 Ibid.

2 Timor.

1 Nouv.-Hollande.

2 Pondichéry.

3 Ibid.

3 Nouv.-Hollande.

2 Madagascar.
Auralus.

Aureus.

Chinensis.

Galbula.

Galbuloides.

Kirrocephalus.

Melanocephalus.

Melanotis.

Moloxila.

Radialus.

Regens.

Virescens.

Viridis.

Xanthonotus.

\section{GENRE ORIOIUS.}

H.ABITAT.

\begin{tabular}{|c|l}
7 & Afrique. \\
1 & Inde. \\
5 & Chine. \\
12 & Europe. \\
$\mathbf{3}$ & Inde. \\
1 & Nouv.-Guinée. \\
7 & Afrique. \\
4 & Timor. \\
1 & Abyssinic. \\
5 & Afrique. \\
$\mathbf{5}$ & Nouv.-Hollande. \\
$\mathbf{2}$ & Inde. \\
7 & Nouv.-Hollande. \\
6 & Ibid.
\end{tabular}

GENRE ORTHOXYY.

Heleroclitus.

Spinicaudus. $\mid$\begin{tabular}{c|l}
2 & Nouv.-Zélande. \\
1 & Nouv.-Hollande.
\end{tabular}

\section{GENRE ORTHOTOMUS.}

Edela.

Flaviventris.

Prinia.

Sepium.

Espèce indéterminėe. 1 Guatimala.

\section{GenRe OXYRAYRCHUS.}

Flammiceps

| 4 | Brésil.

\section{GENRE PACHYCEREALA.}

Gulluralis.

Pectoralis.

Espèce indéterminée. 11 Timor.

\section{GENRE PASTOR.}

Calvus.

Carunculatus.

Cincloramphus.

Contra.

Cristalellus.

Elegans.
2 Philippine.

4 Afrique.

1 Nouv.-Ifollande:

1 Bengale.

2 Java.

1 Cochinchine. 


\begin{tabular}{|c|c|c|}
\hline ESPECES. & 离 & HАBITAT. \\
\hline Ginginianus. & 5 & Java. \\
\hline Griscus. & $\mathbb{1}$ & Toid. \\
\hline Meianoplerus. & 5 & Ibid. \\
\hline Pagodarum. & 1 & Ibid. \\
\hline Roseus. & 6 & Europe, elc. \\
\hline Serex & 3 & Inde. \\
\hline Sericeus. & 1 & Ibid. \\
\hline striatus. & 5 & Bengale. \\
\hline Slurninus. & $\bar{\jmath}$ & Ibia. \\
\hline Temporalis. & 1 & Ibid. \\
\hline Traillii. & 1 & Himalaya. \\
\hline Tristis. & 5 & Bengale. \\
\hline Espèce indéterminée. & 5 & Ibid. \\
\hline
\end{tabular}

\section{GRARE PUIBATERA.}

Flavirostris.

5 Brésil.

\section{GERTE TEGRICUIA.}

Leucocephala.

Rufiventris.

Ruticil!a.

Suecica.

Tilliys.

Plus, zandividus Cormant 叉 especes indétermineses de linde.

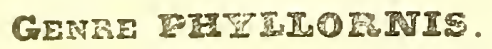

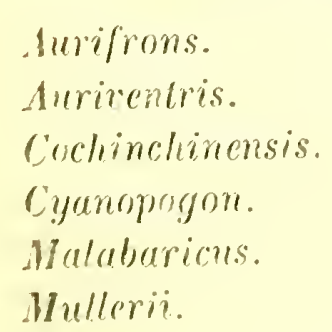

GEMRE PITTA.

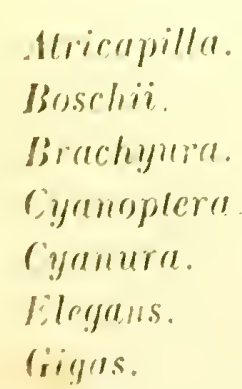

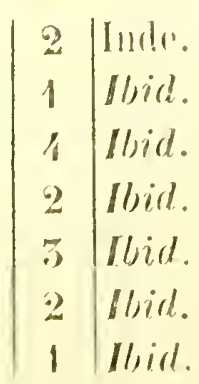

\begin{tabular}{|l|c|l}
\multicolumn{1}{|c|}{ ESPECES. } & $\begin{array}{c}\text { 䓪 } \\
\text { Grallaria. }\end{array}$ & \multicolumn{1}{|c}{ HABITAT. } \\
\hline Granalina. & 2 & Amèrique mér. \\
Irena. & 2 & Inde. \\
Sirepilans. & 2 & Ibid. \\
Tiniens. & 2 & Nouv.-Hollande. \\
Venusla. & 1 & Guiane. \\
& 2 & Sumatra.
\end{tabular}

Plus, 9 individus formant 4 espèces indéterminces ou inćdites provenant de diverses localites.

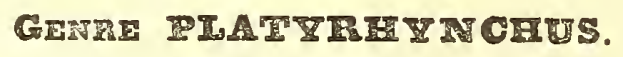

Cancromus.

Olivaceus.

$$
\begin{array}{l|l}
2 & \text { Brésil. } \\
& \text { Ibid. }
\end{array}
$$

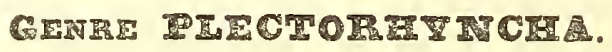

Lancolala.

| 1 |Nouv.-Hollande.

\section{CENRE DOTA}

Isidori.

Tlontonus.

Supercitiosus.

Trivirgalus.

$$
\begin{array}{|l|l}
2 & \text { Nouv.-Hollande. } \\
3 & \text { Java. } \\
5 & \text { Nouv.-Hollande. } \\
2 & \text { Ibid. }
\end{array}
$$

Plus, 6 individus formant 3 espéces indéterminćes ou inédites provenant de la NouvelleIloliande.

\section{GENRB PREOROPS.}

Crislatus.

Geoffroyi.

Plumalus.

Talacoma.

\begin{tabular}{|l|l}
1 & Afrique. \\
1 & Hbid. \\
2 & Hbid. \\
2 & Lbid.
\end{tabular}

\section{GENRE TSAIIIS.}

Atricapilla.

Auranious.

Castaneus.

Cayanus.

Crislalus.

Cuvieri.

IIubirl.

Jardinii.

\begin{tabular}{|l|l}
3 & Amérique. \\
1 & Cayenne. \\
2 & Amérique. \\
2 & Cayenne. \\
2 & Amérique. \\
2 & Ibid. \\
1 & Ibid. \\
5 & Brésil.
\end{tabular}




\begin{tabular}{|c|c|c|}
\hline ESPECES. & 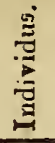 & HABITAT. \\
\hline Navius. & 3 & Cayenne. \\
\hline Niger. & 2 & Brésil. \\
\hline Personatus. & 2 & Cay enne. \\
\hline Pilealus. & 3 & Amérique. \\
\hline Roseicollis. & 4 & Ibid. \\
\hline Rufescens. & 4 & Ibid. \\
\hline Vieillolii. & 2 & Ibid. \\
\hline
\end{tabular}

Plus, 12 individus formant 8 especes indéterminées ou inédites d'Amérique.

\section{GENRE PSAROCOIIUS.}

Albirostris.

Anticus.

Baltimore.

Banana.

Bifascialus.

Bullockii.

Caslaneus.

Cayennensis.

Chrysocephalus.

Chrysoplerus.

Crislalus.

Croconotus

Cucullalus.

Diadematus.

Flavigaster.

Flavus.

Fringillarius.

Froncalis.

Guirahuro.

Gubernalor.

Gularis.

Gymnops.

Homorrhous.

Icterocephalus.

Icteronotus.

Jamacaii.

Melanocephalus.

Mesomelas.

Militaris.

Montesuma.

Niger.

Nigerrimus.

Palliatus.

Pecoris.

\begin{tabular}{|c|c|}
\hline 7 & |Brésil. \\
\hline 2 & Ibid. \\
\hline 8 & Ibid. \\
\hline & Antilles. \\
\hline & Brésil. \\
\hline 1 & Amérique sept. \\
\hline 9 & Ibid. \\
\hline & Amérique. \\
\hline & Brésil. \\
\hline & Ibid. \\
\hline & Ibid. \\
\hline & Ibid. \\
\hline & Mexique. \\
\hline & Ibid. \\
\hline & Amérique. \\
\hline & Ibid. \\
\hline & Ibid. \\
\hline & Ibid. \\
\hline & Ibid. \\
\hline & Ibid. \\
\hline & Mexique. \\
\hline 9 & Cayenne. \\
\hline 6 & Amérique. \\
\hline & Ibid. \\
\hline & Ibid. \\
\hline 5 & Ibid. \\
\hline & Mexique. \\
\hline & Ibid. \\
\hline 1 & Amérique. \\
\hline & Mexique. \\
\hline & Saint-Domingue. \\
\hline & Brésil. \\
\hline & Amérique. \\
\hline & Ibid. \\
\hline
\end{tabular}

\begin{tabular}{|c|c|c|}
\hline ESPÈCES. & 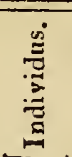 & HABITAT. \\
\hline Phœniceus. & 14 & Amérique. \\
\hline Pustulalus. & 5 & Mexique. \\
\hline Pyrrıopterus. & 3 & Amérique. \\
\hline Sericeus. & 7 & Ibid. \\
\hline Sülcirostris. & 5 & Ibid. \\
\hline Tricolor. & 1 & Ibid. \\
\hline Viridis. & 4 & Cayenne. \\
\hline Xanthocephalus. & $\check{\jmath}$ & Amérique sept. \\
\hline Xanthornis. & 9 & Cayenne. \\
\hline
\end{tabular}

Plus, $\mathbf{3 1}$ individus formant $\mathbf{2 0}$ espèces ind terminées ou inédites provenant de diverses localités.

\section{GenRE PSILOPUS.}

Albogularis.

Brevirostris.

Olivaceus.

$\mid$\begin{tabular}{c|l}
1 & Nouv.-Hollande. \\
4 & Ibid. \\
1 & Ibid.
\end{tabular}

\section{GENRE PSOPHODES.}

Crepilans.

| 4 |Nouv.-Hollande.

\section{GENRE PYRRHOCORAK.}

Graculus.

Pyrrhocorax.

$$
\mid \begin{array}{l|l}
2 & \text { Europe, etc. } \\
2 & \text { Ibid. }
\end{array}
$$

\section{GenRE QUERUIA.}

Cinerea.

Purpurea.

Rubricollis.

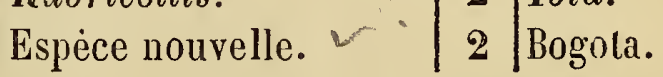

\section{GenRe Quiscatus.}

Ferrugineus.

| 1 |Amérique sept.

Plus, 22 individus formant 10 espéces indéterminées ou inédites de diverses localités.

\section{GERRz REGUTUS.}

Calendula.

Crislalus.

Ignicapillus.

Modestus.

Vulgaris.
5 Amérique sept.

2 Ibid.

4 Europe

1 Ibid. 


\begin{tabular}{|c|c|c|}
\hline ESPËCES. & 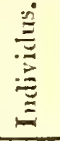 & HABITAT. \\
\hline \multicolumn{3}{|c|}{ GENRE BIPIDURA. } \\
\hline Albiscapa. & 2 & Nouv.-Ilollande. \\
\hline Euryura. & 5 & Jara. \\
\hline Perspicillata. & 5 & Afrique mérid. \\
\hline Ruficauda. & 2 & Nouv.-Hollande. \\
\hline Rufifrons. & 1 & Ibid. \\
\hline
\end{tabular}

Plus, 14 individus formant 9 espèces indéterminées ou inédites provenant de diverses localités.

\section{GENRE IUPICOTA.}

Aurantia.

Peruciana.

Viridis.

$$
\mid \begin{array}{l|l}
6 & \text { Cayenne. } \\
5 & \text { Amérique mér. } \\
5 & \text { Sumatra. }
\end{array}
$$

\section{GENRE SA ICOIA.}

Albifrons.

Albiscapulata.

Albospecularis.

Aurita.

Bicolor.

Bifasciata.

Cachinnans.

Caprala.

Coruphous.

Deserti.

Familiaris.

Formicivora.

Leucomela.

Lugens.

Lugubris.

Melanoleuca.

Melanoptera.

Monaclia.

Moniana.

olinanthe.

rileata.

Plygmalura.

Riubetra.

Rubicola.

Rubricola.

Ruficentris.

Rufocinerea.

\begin{tabular}{l|l}
1 & Abrssinie. \\
4 & Ibid. \\
1 & Madagascar. \\
3 & Europe. \\
1 & Nour.-Hollande. \\
2 & Afrique. \\
5 & Europe. \\
8 & Inde. \\
5 & Afrique. \\
1 & Egypte. \\
1 & Afrique. \\
2 & Ibid. \\
5 & Europe. \\
2 & Ibid. \\
2 & Afrique. \\
1 & Timor. \\
1 & Ibid \\
1 & Nubie. \\
6 & Afrique. \\
12 & Europe. \\
4 & Afrique. \\
6 & Inde. \\
4 & Amerique. \\
13 & Europe. \\
3 & Afrique. \\
2 & Ibid. \\
1 & Ibid. \\
1 & Ancriquc. \\
&
\end{tabular}

\begin{tabular}{|c|c|c|}
\hline ESPÈCES. & & HABITAT. \\
\hline Solilaria. & 1 & Nour.-Hollande. \\
\hline Sordida. & 1 & Afrique. \\
\hline Sperata. & 1 & Ibid. \\
\hline Slapazina. & 4 & Europe. \\
\hline Stygmalura. & Јั & Afrique. \\
\hline
\end{tabular}

Plus, 12 indiridus formant 8 espèces indéterminées ou inédites de diverses localités.

\section{GERE SERICORNIS.}

Citreogularis.

1 | Nour.-Hollande.

\section{GanRE STRUTHIDE.}

Cinerea.

2 Kouv,-Hollande.

\section{GENRE STIVIA.}

Esliva.

Americana.

Aquatica.

Arundinacea.

Alricapilla.

Alriceps.

Aurocapilla.

Aurorea.

Aulumnalis.

Asaria.

Babacola.

Berticata.

Blackburnice.

Brachyplera.

Carulea.

Canadensis.

Cayana.

Castanea.

Celti.

Chloronotus.

Cinerea.

Cisticola.

Conspicillata.

Coronala.

Curruca.

Cyanocephala.

Delicalula.
8 Amérique sept.

4 Japon.

6 Amérique sept.

5 Europe.

Ibid.

6 Carenne.

4 Mexique.

2 Amérique sept.

2 Japon.

2 Amérique sept.

1 Ibid.

ฮั Afrique.

3 Ibid.

5 Amérique sept.

1 Afrique.

4 Cayenne.

5 Amérique sept.

o Caỵenne.

2 Amérique sept.

2 Europe.

1 Nour-Hlollande.

8 Europe.

6 Ibid.

2 Ibid.

5 Amérique sept.

5 Europe.

8 Cayenne. 


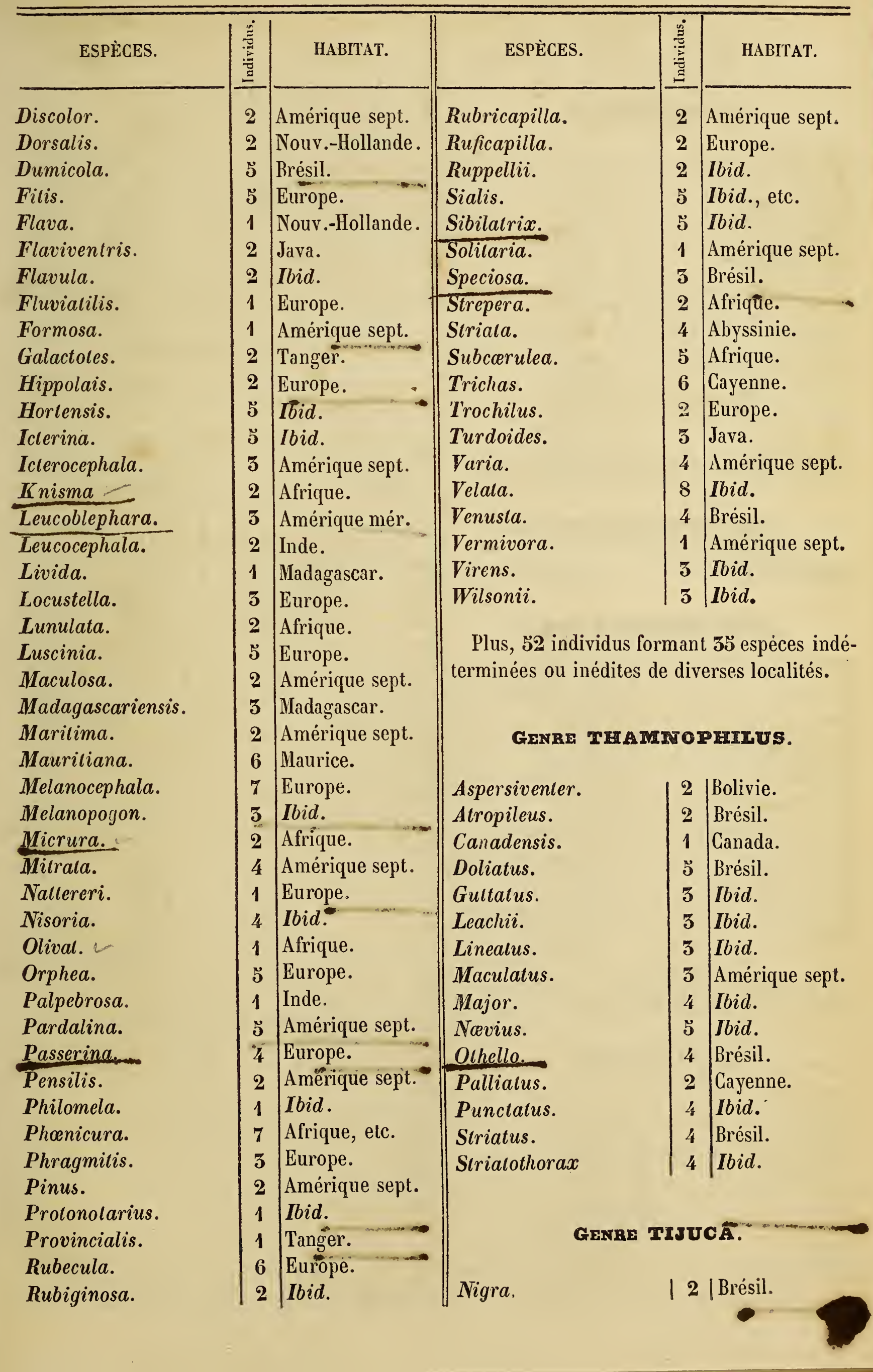




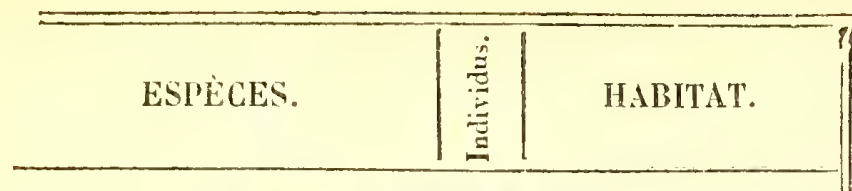

\section{GENRE IIIIIAIA.}

Gularis.

Horsficldii.

Hyperyihra.

Hypoleuca.

Lepidocephala.

Maculala.

Nigricollis.

Pileala.

Pacilorhyncha.

Poliocephala.

Pyrrhoplera.

Thoracica.

Trichorrhos.

\begin{tabular}{|c|l|}
3 & Inde. \\
2 & Ibid. \\
5 & Ibid. \\
1 & Ibid... \\
1 & Ibid. \\
2 & Ibid. \\
2 & Ibid. \\
5 & Ibid. \\
1 & Ibid. \\
1 & Ibid. \\
1 & Ibid. \\
3 & Ibid. \\
2 & Ibid.
\end{tabular}

\section{GENRE TRICHORFORUS.}

Importunus.

( Afrique.

\section{GERE TIROGIODYTES.}

Bewickii.

Cantans.

Coraya.

Fumigatus.

Gularis.

Longirostris.

Ludovicianus.

Luscinius.

Mexicanus.

Platensis.

Vulgaris.

\begin{tabular}{c|l}
1 & Mexique. \\
5 & Cayenne. \\
5 & Ibid. \\
1 & Europe. \\
7 & Anérique. \\
5 & Ibid. \\
1 & Afrique. \\
1 & Océanie. \\
2 & Mexique. \\
6 & Amérique mér. \\
7 & Europe.
\end{tabular}

Plus, 19 individus formant 8 espèces indéterminces ou inedites de diverses localites.

GenRe turdus.

\begin{tabular}{l|l|l} 
Albicapilla. & 3 & Arrique. \\
Albiceps. & 2 & Ibid. \\
Albicollis. & 2 & Brésil. \\
Albovillatus. & 1 & Ibid. \\
Amaurolis. & 2 & Jipon. \\
Aqualicus. & 3 & Arnérique sept. \\
Ardesiacus. & 4 & Bresil. \\
Arsino (Ixos). & 4 & Abyssinir.
\end{tabular}

Alriceps (Ixos!. Aurigaster.

Azureus (Ixos).

Bimaculalus (Ixos).

Brasiliensis.

Cafer (Ixos).

Calandria.

Capensis.

Cardis.

Chiguaneo.

Chrysolaus.

Cinclorhynchus.

Cilvinus.

Cyanolus.

Cyanus.

Dispar (Ixos).

Edolioides.

Erythroplerus.

Erylhrorhynchus.

Exploralor.

Fulklandia.

Felivox.

Fiscater.

Fuscalus.

Gilvus.

Griseiceps.

Hispaniolensis.

Iliacus.

Indicus.

Interpres.

Jocosus.

Libonyana.

Longirostris.

Macrourus.

Madagascariensis.

Manillensis.

Melanotis.

Merula.

Migralorius.

Minor.

Musicus.

Mutabilis.

Naumannii.

Nestor.

Nigricans.
HABITAT.

Java.

Afrique.

Sumatra.

Ibid.

Brésil.

Afrique.

Amérique mér.

Afrique.

Japon.

Pérou.

Japon.

Ilimalaya.

Java.

Inde.

Europe.

Java.

Afrique.

lbid.

lbid.

lbid.

Malouines.

Amérique sept.

Amérique mér.

Antilles.

Amérique sept.

Inde.

Ibid.

Europe.

Java.

2 Ibid.

3 Ibid.

Afrique.

2 Mexique.

4 Inde.

4 Madagascar.

6 Philippines.m.

2 Mexique.

9 Europe.

6 Amérique sept.

3 Europe, etc.

3 Ibid.

4 Java.

2 Europe, elc.

5 Nouv.-Hollande.

8 Afrique. 


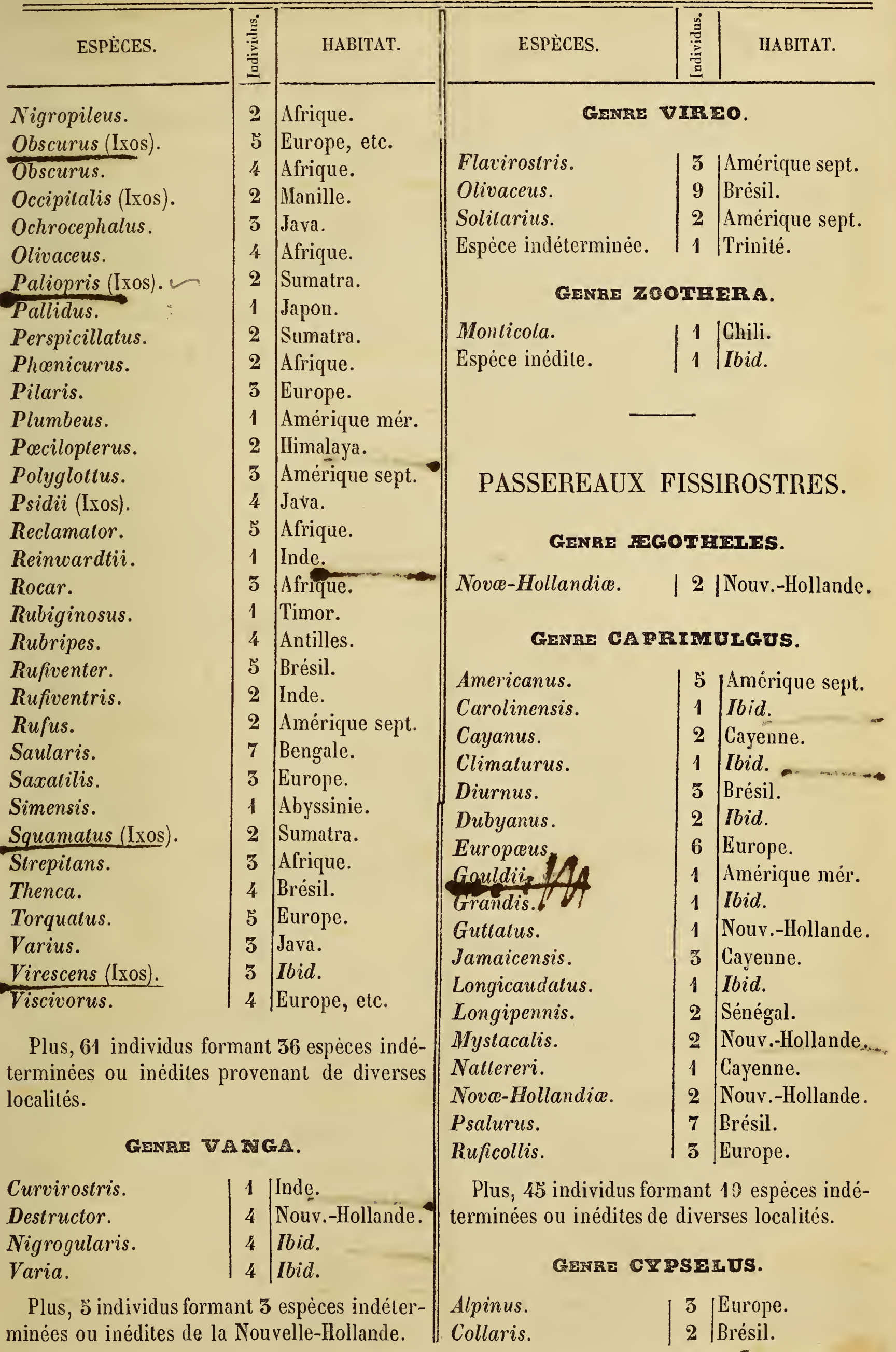




\begin{tabular}{|c|c|c|}
\hline ESPECES. & 总 & HABITAT. \\
\hline $\begin{array}{l}\text { Comalus. } \\
\text { Francice. } \\
\text { Leuconolus. } \\
\text { Longipennis. } \\
\text { Murarius. } \\
\text { Pygargus. } \\
\text { Spinicaudus. } \\
\text { Enicolor. }\end{array}$ & $\begin{array}{l}2 \\
4 \\
4 \\
1 \\
2 \\
2 \\
2 \\
2 \\
2\end{array}$ & $\begin{array}{l}\text { Sumatra. } \\
\text { Maurice. } \\
\text { Inde. } \\
\text { Java. } \\
\text { Europe. } \\
\text { Afrique. } \\
\text { Cayenne. } \\
\text { Mradagasear. }\end{array}$ \\
\hline
\end{tabular}

Plus, 4 individus formant 5 especes indẻterminées de Colombie.

\section{GMNRE HIRENDO.}

Americana.

Boissoneautii.

Capensis.

Cayanensis.

Dominicensis

Esculenia.

Fasciata.

Fulva.

Jaranica.

Jugularis.

Leucoptera.

$\frac{\text { Mimula. }}{\text { Paludicola. }}$

Purpurea.

Riparice.

Rufifrons.

Rufogularis.

Rupestris.

Rustica.

Rusticoides.

Senegalensis

Strioluta.

Thalassina.
Torguala.

Urbica.

\begin{tabular}{|c|c|}
\hline 5 & |Cayenne. \\
\hline 5 & Europe. \\
\hline 2 & M fripue. \\
\hline 1 & Cayenne. \\
\hline 4 & Mid. \\
\hline 4 & Philippines. \\
\hline 5 & Cayenne. \\
\hline 2 & Amérique. \\
\hline 2 & Java. \\
\hline 5 & Brésil. \\
\hline 3 & Cayenue. \\
\hline 7 & Brésil. \\
\hline 4 & Arrique. \\
\hline 5 & Amérique sept. \\
\hline 8 & Europe. \\
\hline 2 & Alipine: \\
\hline 1 & Ibid. . \\
\hline 2 & Europe. \\
\hline 5 & Ibid. \\
\hline 2 & Java. \\
\hline 2 & Afrique. \\
\hline 2 & Jiva. \\
\hline 1 & Hexique. \\
\hline 1 & Aliqque. \\
\hline & Europe, etc. \\
\hline
\end{tabular}

Plus, 35 individus formant 19 especes indérermines ou inedites de diverses localités.

\section{GENR PODARGUS.}

\section{Cornulus.}

Humeralis.

Espece inditermince.
1 Java.

5 Nomv.-Hollinde.

1 Ibid.
ESPCECES.

HABITAT.

\section{GENRE STEATORNIS.}

Caripensis.

1 IColombie.

\section{PASSEREAUX CONIROSTRES.}

\section{Gemre AIAUDA.}

Alpestris.

Apiala.

Arbores.

Arvensis.

Bilopha.

Brachydactyle.

Calandra.

Capensis.

Cineroides.

Crassirostris.

Cristala.

Crocea.

Ferruginea.

Ellavicollis.

Isabellina.

Mirafra.

Ruficeps.

Talarica.

\begin{tabular}{|c|l|}
4 & Europe. \\
4 & Afrique. \\
4 & Europe. \\
9 & Ibid. \\
4 & Nubie. \\
6 & Europe. \\
2 & Ibid. \\
2 & Afrique. \\
5 & Ibid. \\
2 & Ibid. \\
3 & Europe. \\
3 & Afrique. \\
1 & Ibid. \\
2 & Ibid. \\
2 & Arabie. \\
7 & Inde. \\
1 & Afrique. \\
1 & Europe.
\end{tabular}

Plus, 12 individus formant 8 especes indé:terminėes ou inédites de diverses localités.

\section{GENRE ASTEAPIA.}

Gularis.

| 2 |Nouv.-Guinée.

\section{GENRE BUPIAGA.}

Africana.

Erylleroryncha.

$\mid$\begin{tabular}{l|l}
2 & Afrique. \\
2 & Ibid.
\end{tabular}

\section{GENRE CERTIIIAUDA.}

Africana.

Albofasciala.

Bifasciala.

Nivosa.

Rufopalliala.

\begin{tabular}{|l|l}
4 & Afrique. \\
2 & Ibid. \\
2 & Ibid. \\
2 & Ibid. \\
1 & Ibid.
\end{tabular}

Plus, 3 individus formant 5 espèces indéterminées on inédites d'Afrique. 


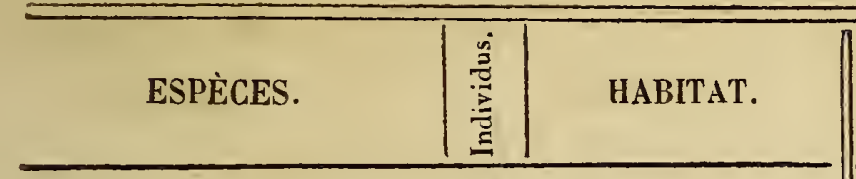

Genre cotaris.

Cyanicollis.

Gularis.

Leplosomis.

Madagascariensis.

Purpurascens.

\begin{tabular}{|l|l}
5 & Inde. \\
1 & Afrique. \\
1 & Madagascar. \\
1 & Ibid. \\
2 & Afrique.
\end{tabular}

\section{GenRe CORACIAS.}

Angolensis.

Caudata.

Cyanogaster.

Garrula.

Indica.

Pilosa.

Temminkii.

Plus, $\mathbf{3}$ individus formant $\mathbf{2}$ espèces indéterminées ou inédites.

\section{Genre corvus.}

Affinis.

Albicollis.

Azureus.

Beechii.

Bispecularis.

Bollanensis.

Bullockii.

Canadensis.

Cayanus.

Corax.

Cornix.

Corone.

Crassirostris.

Crislatellus.

Crislalus.

Cyaneus.

Cyanomelas.

Cyanopogon.

Enca.

Erythrorhynchos.

Floridanus.

Frugilegus.

Fuliginosus.

Galericulalus.

Glandarius.

\begin{tabular}{|l|l}
$\mathbf{2}$ & Afrique. \\
$\mathbf{3}$ & Ibid. \\
$\mathbf{2}$ & Java. \\
$\mathbf{4}$ & Europe. \\
$\mathbf{3}$ & Afrique. \\
$\mathbf{3}$ & Ibid., elc. \\
$\mathbf{3}$ & Inde.
\end{tabular}

\begin{tabular}{|l|l|}
$\mathbf{2}$ & Abyssinie. \\
$\mathbf{2}$ & Afrique.
\end{tabular}

3 Brésil. 。

2 Afrique..

2 llimalaya.

1 Inde.

2 Vexique.

2 Amérique sept.

2 Cayenne.

4 Europe.

4 Ibid.

7 Ibid.

2 Abyssinie.

1 Amèrique mér.

4 Amérique sept.

3 Europe.

2 Amérique mér.

4 Mexique.

2 Abyssinie.

2 Himalaya.

4 Amérique sept.

2 Europe.

5 Mexique

4 Java.

4 Europe, etc.
Gubernulrix.

Infaustus.

Japonicus.

Lanceolatus.

Leucolophus.

Levaillantii.

Macropterus.

Macrorhynchos.

Monedula.

Ossifragus.

Perspicillatus.

Pica.

Pileatus.

Rufiginosa.

Scapulatus.

Senex. wt

Splendens.

Stelleri.

Striatus.

Torquatus

Uleramarinus.

Viridicyanus.
Peruvianus.
HABITAT.

Mexique.

3 Europe.

Japon.

3 llimalaya.

2 Ibid.

2 Afrique.

2 lbid.

4 Océanie.

2 Europe.

2 Amérique sept.

3 Java.

3 Bogota.

4 Europe.

ร Amérique nér.

5 Mexique.

3 Afrique.

1 Nouv.-Guinée.

3 Inde.

2 Amérique sept.

2 Bengale.

3 Calédonie.

2 Amérique sept.

2 Bogota.
Plus, 14 individus formant 8 espéces indẻterminées ou inédites de diverses localités.

\section{GRRE CRYPTORHINA.}

Piapiac.

Pecilorhynchos.

Varians.

\begin{tabular}{l|l}
2 & Afrique. \\
2 & Java. \\
$\mathbf{3}$ & Afrique.
\end{tabular}

\section{GENRE DEITDEOCITTA.}

Leucogaster.

Occipilalis.

Rufiventris.

Sinensis.

\begin{tabular}{|l|l}
$\mathbf{2}$ & Inde. \\
$\mathbf{2}$ & Ibid. \\
$\mathbf{2}$ & Himalaya. \\
$\mathbf{2}$ & Ibid.
\end{tabular}

\section{GenRe GLAUcopis.}

Cinerea.

Leucoptera. \begin{tabular}{|l|l}
2 & Nouv.-Hollande. \\
4 & Sumatra.
\end{tabular}

\section{GRNRE TUCTERAGA.}

Caryocatactes.

Hemispila 


\begin{tabular}{|c|c|c|}
\hline ESI'ECES. & 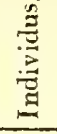 & HABITAT. \\
\hline \multicolumn{3}{|c|}{ GENRE PARADISEA. } \\
\hline Apoda. & 2 & Nouv.-Guinée. \\
\hline Magnifica. & 1 & Ibid. \\
\hline Minor. & 5 & Ibid. \\
\hline Regia. & 2 & Ibid. \\
\hline Rubra. & 1 & Ibid. \\
\hline Sexsetacea. & 1 & lloid. \\
\hline Superba. & 1 & Ilbid. \\
\hline
\end{tabular}

GENRE STURT

\begin{tabular}{|c|c|c|}
\hline Ludovicianus. & b’ & Amèlique sept. \\
\hline Militaris. & I & Amérique mér. \\
\hline Pyrrhocephalus. & $g$ & Ibid. \\
\hline Unicolor. & 2 & Europe. \\
\hline Vulgaris. & 7 & Ibids= \\
\hline
\end{tabular}

\section{PASSEREAUX TÉNUIROSTRES.}

\section{GENRE AINARATIS.}

Albogularis.

Amaurolis.

Aradoides.

Cristalus.

Leucophris.

Ruficollis.

Rufifrons.

Squamiger.

Siriolatus.

Superciliaris.

Unirufus.

Plus, 18 individus formant 10 especes indéterminées ou inédites du Brésil.

\section{GENRE ARACHNOTHERA.}
Chrysogenys.
Inornata.
Longirostris.

$\mid$\begin{tabular}{l|l}
2 & Javal. \\
3 & Ibid. \\
2 & Ibid.
\end{tabular}

\section{GENRE CFEREBA.}

\begin{tabular}{l|l|l} 
Corulea. & $\mathbf{6}$ & Cayenne. \\
Cyamea. & 1 & Ibid. \\
Flarenla. & 4 & Brésil. \\
Spiza. & 9 & Cayenne.
\end{tabular}

ESPÈCES。

HABITAT.
Familiaris.

| 7 |Europe.

\section{GENRE CIIMACIERIS.}

Erylhrops.

Picumnus.

Scandens.

$$
\mid \begin{array}{l|l}
1 & \text { Nour.-Hollande. } \\
5 & \text { Ibid. } \\
1 & \text { Ibid. }
\end{array}
$$

\section{GENRE DIITDIOCOUARTES.}

Albicollis.

Atrirostris.

Bivitlalus.

Cayennensis.

Cuneatus.

Falcirostris.

Fuliginosus.

Gullatus.

Lessonii.

Lcvaillantii.

Nasicus.

Picus.

Procurvus.

Sylviellus.

\begin{tabular}{|c|l}
5 & Amérique mér. \\
1 & Bogata. \\
4 & Amérique mér. \\
1 & Cayenne. \\
5 & Ibid. \\
2 & Brésil. \\
2 & Ibid. \\
12 & Ibid. \\
1 & Ibid. \\
2 & Ibid. \\
1 & Cayenne. \\
4 & Brésil. \\
5 & Cayenne. \\
2 & Brésil.
\end{tabular}

Plus, 5 individus formant 2 espéces indéterminées du brésil.

\section{GRRR DIC REUTR.}

Cantilans.

Hirundinaceum.

Pecloralis.

Rubrocanum.

Rufescens.

Sanguinolentum.

\begin{tabular}{|l|l}
2 & Nouv.-Hollande. \\
3 & Ibid. \\
$\mathbf{3}$ & Java. \\
4 & Nouv.-IIollande. \\
1 & Ibid. \\
5 & Java.
\end{tabular}

Plus, 8 individus formant 2 espèces indéterminces de Malacca.

\section{GENRE DERPAIS.}

Vestiaria.

Espéce indéterminée. $\mid \begin{aligned} & \text { 4 } \\ & \text { Ibid. }\end{aligned}$ 


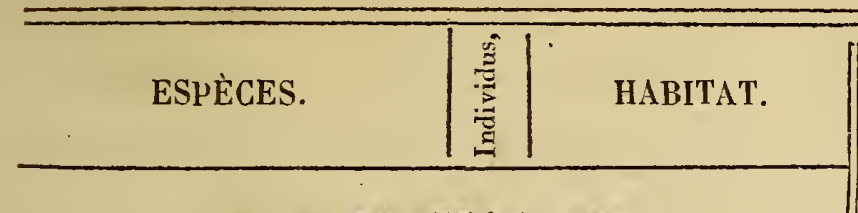

GENRE RPIMACEUS.

\begin{tabular}{l|l|l} 
Albus. & 1 & Nouv.-Guinée. \\
Cyanomelas. & 4 & Afrique. \\
Drepanorhynchus. & 1 & Ibid. \\
Erythrorhynchus. & 6 & Ibid. \\
Magnificus. & 1 & Nouv.-Guinée. \\
Melanorhynchus. & 4 & Afrique. \\
Pusillus. & 5 & Ibid. \\
Regius. & 5 & Noüv.-Guinée.
\end{tabular}

GENRE FAIGUIIA.

Palliala.

12 |Madagascar.

GENRE ITECTARINIA.

Adelberti.

Enea.

Affinis.

Amaurotis.

Aspasia.

Auratifrons.

Chalybea.

Chrysogenys.

Coccinigaster.

Collaris.

Currucaria.

Cyanocephala.

Eximia.

Famosa.

Fusca.

Gouldion.

Hassellii.

Inornala.

Kuhlii.

Lepida.

Longirosiris.

Longuemarei.

Lolenia.

Luleovenler.

Madagascariensis.

Melallica.

Mystacallis.

Nibara.

Nigra.
2 Afrique.

5 Nouv.-Hollande.

\begin{tabular}{|c|c|c|}
\hline ESPËCES. & 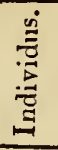 & HABITAT. \\
\hline Olivacea. & 1 & Afrique. \\
\hline Pectoralis. & 1 & Inde. \\
\hline Phœnicotis. & 4 & Ibid. \\
\hline Platura. & 1 & Afrique. \\
\hline Promerops. & 2 & Ibid. \\
\hline Prolea. & 5 & Abyssinie. \\
\hline Pulchella. & 6 & Ibid. \\
\hline Purpurala. & 7 & Afrique. \\
\hline Senegalensis. & 2 & Ibid. \\
\hline Solaris. & 5 & Inde. \\
\hline Sperala. & 4 & Ibid. \\
\hline Splendens. & 5 & A frique. \\
\hline Splendida. & 3 & Nouv.-Hollande. \\
\hline Superciliosa. & 2 & Ibid. \\
\hline Tacazze. & 3 & Abyssinie. \\
\hline Talatala. & 3 & Ibid. \\
\hline Temminkii. & 1 & Brésil. \\
\hline Tenuirostris. & 1 & Nouv.-Hollande. \\
\hline Verreauxii. & 2 & Afrique. \\
\hline Violacea. & $\check{\jmath}$ & Inde. \\
\hline
\end{tabular}

Plus, 14 individus formant 5 espèces indéterminées ou inédiles d'Afrique et de l'Océanie.

\section{GENRE OPITIORHYNCHUS.}

Anumbi.

\begin{tabular}{l|l|l} 
Brunneicapillus. & 2 & Ibid.
\end{tabular}

Ruber. $\quad 2$ lbid.

Rufus.

Roseus.

Scolopaceus.

Strialiceps.

Striaticollis.

\begin{tabular}{l|l|l} 
Zonalus. & $\mathbf{2}$ & Ibid. \\
Espèce indéterminée. & 4 & Chili.
\end{tabular}

\section{GETRE STTTA.}

Canadensis.

Carolinensis.

Chrysoplera.

Europaa.

Melanocephala.

Pileala.

Syriaca.

Velala.

2 Amérique sept.

3 Ibid.

2 Nouv.-Hollande.

10 Europe.

1 Nouv.-Hollande.

1 Amérique.

2 Europe.

2 Amérique sept.

Plus, 2 individus indėterminés d'Amérique. 


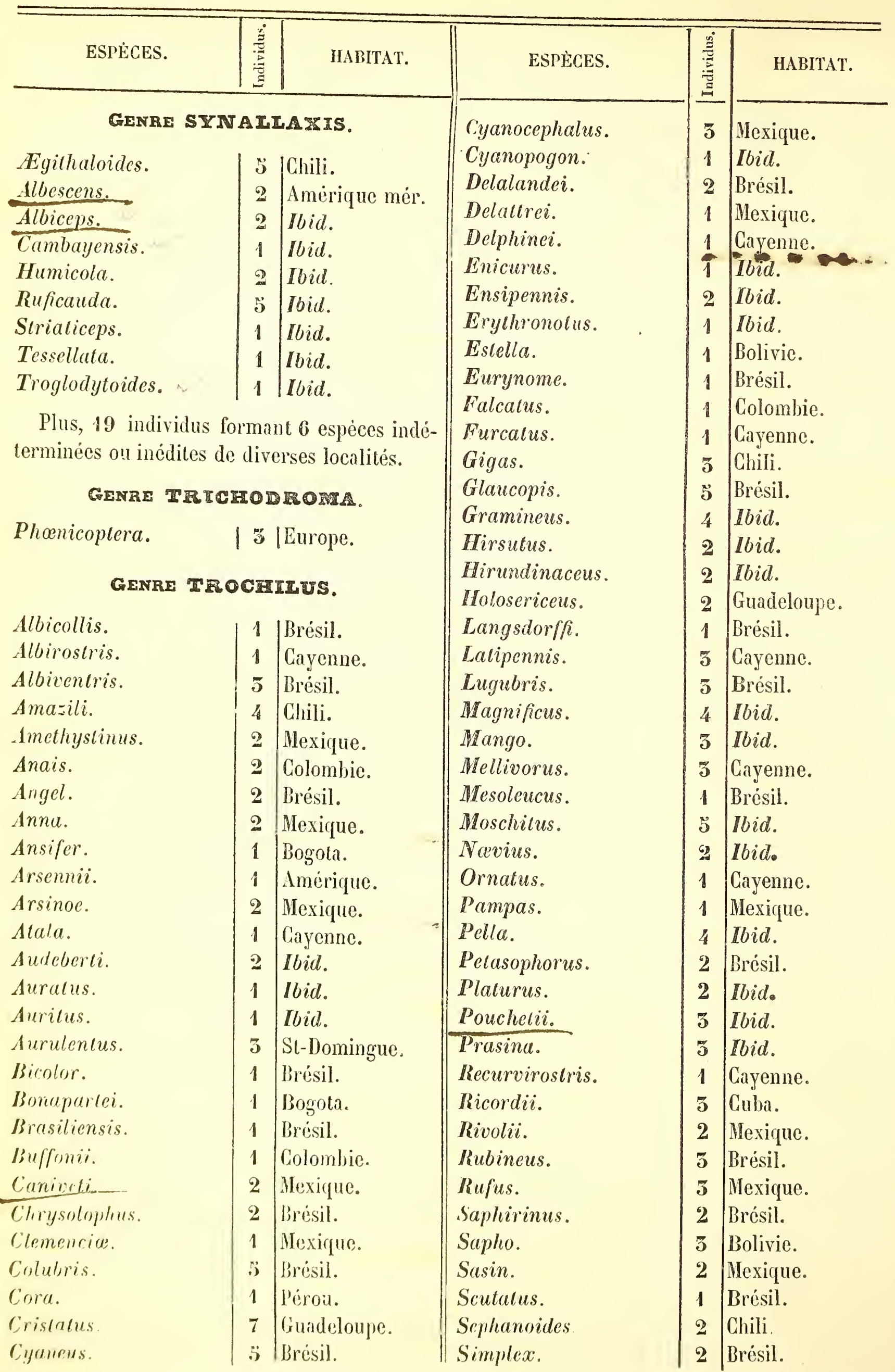




\begin{tabular}{|c|c|c|}
\hline ESPÈCES. & 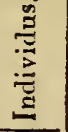 & HABI'TAT. \\
\hline Superbus. & 1 & Mexique. \\
\hline Superciliosus. & 1 & Cayenne. \\
\hline Temminkii. & 2 & Bogota. \\
\hline Vesper. & 2 & Chili. \\
\hline Vestila. & 2 & Bogota. \\
\hline Vieillolii. & 3 & Brèsil. \\
\hline Viridis. & 2 & Cayenne. \\
\hline Viridissimus. & 3 & Ibid. \\
\hline
\end{tabular}

\section{GENRE UNCIROSTRUM.}

\begin{tabular}{l|l|l} 
Albilaterum. & 6 & Bogota. \\
Carbonarium. & 1 & Brésil. \\
Cyaneum. & 5 & Bogota. \\
Lafresnayei. & 2 & Ibid. \\
Orbignyi. & 3 & Ibid. \\
Siltoides. & 2 & Mexique.
\end{tabular}

\section{GENRE UPPUCERTHIA.}

Vulgaris.

| 6 |Amérique mér.

Plus, 3 individus formant 2 espèces indéterminées d'Amérique.

\section{GENRE URUPA.}

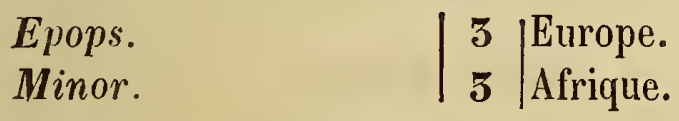

\section{GENRE XIRTOPS.}

Anabatoides.

Genibarbis.

$\boldsymbol{R u f o s u p e r c i l i a t u s .}$

\begin{tabular}{|c|l}
2 & Brésil. \\
1 & Ibid. \\
5 & Ibid. \\
2 & Ibid.
\end{tabular}

Rutilans.

Plus, 2 individus formant $\mathbf{2}$ espéces indéterminėes du Brésil.

\section{PASSEREAUX SYNDACTYLES.}

\section{Gente ALEDo.}

Alcyon.

Americana.

Asialica.

Atricapilla.

\begin{tabular}{l|l}
4 & Amérique. \\
8 & Ibid. \\
2 & Asie. \\
$\mathbf{3}$ & Inde.
\end{tabular}

\begin{tabular}{|c|c|c|}
\hline ESPĖCF.S. & 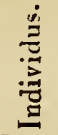 & HABITAT. \\
\hline Bicolor. & 4 & Inde. \\
\hline Biru. & 1 & Ibid. \\
\hline Cancrophaga. & 1 & Afrique. \\
\hline Capensis. & 1 & Ibid. \\
\hline Chlorocephala. & 4 & Ibid. \\
\hline Coromanda. & 3 & Inde. \\
\hline Cristata. & 6 & Cayenne. \\
\hline Cyanopectoralis. & $\mathbf{3}$ & Sénégal. \\
\hline Cyanosligma. & 2 & Afrique. \\
\hline Cyanotis. & 4 & Java. \\
\hline Dea. & 1 & Trinité. \\
\hline Diops. & 1 & Sumatra. \\
\hline Eryıhrogaster. & 10 & Afrique. \\
\hline Hispida. & $\mathfrak{5}$ & Europe. \\
\hline Hispidula. & 4 & Inde. \\
\hline Lugubris. & 2 & Himalaya. \\
\hline Macleayii. & 1 & Nuuv.-Hollande. \\
\hline Maxima. & 4 & Afrique. \\
\hline Melanorhyncha. & 1 & Ibid. \\
\hline Meninting. & 4 & Inde. \\
\hline Omnicolor. & 2 & Ibid. \\
\hline Pygmжа. & 4 & Atrique. \\
\hline Rudis. & 4 & Ibid. \\
\hline Sacra. & 7 & Nouv.-Zélande. \\
\hline Semicarulea. & 2 & Ibid. \\
\hline Semilorquala. & $\mathbf{3}$ & Afrique. \\
\hline Senegalensis. & 5 & Ibid. \\
\hline Smyrnensis. & 6 & Inde. \\
\hline Superciliosa. & 4 & Cayenne. \\
\hline Torquata. & 8 & Brésil. \\
\hline Veslita. & 6 & Ibid. \\
\hline Vinlsioides. & 2 & Madagascar. \\
\hline
\end{tabular}

\section{GENRE BUCEEOS.}

\begin{tabular}{|||l|l|l} 
Abyssinicus. & 4 & Afrique. \\
Anlracicus. & 1 & Java. \\
Alralus. & 1 & Ibid. \\
Bicornis. & 2 & Ibid. \\
Buccinator. & 2 & Afrique. \\
Cassidix. & 2 & Cèlèbes. \\
Cineraceus. & 3 & Inde. \\
Comatus. & 1 & Sumatra. \\
Convexus. & 2 & Ibid. \\
\hline Coronalus. & 2 & Afrique.
\end{tabular}




\begin{tabular}{|c|c|c|c|c|c|}
\hline ESPÈCES. & 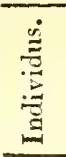 & IABITAT. & ESPĖCES. & & HABITA'T. \\
\hline Cristatus. & 2 & Afrique. & Bullockii. & $\xi$ & Afrique. \\
\hline Erythrorhynchus. & 6 & Ibid. & Bullockoides. & 2 & Ibid. \\
\hline Exarhatus. & 4 & Célèbes. & Chrysolaimus. & 1 & Ibid. \\
\hline Fascialus. & 1 & Afrique. & Cuvieri. & 3 & Ibid. \\
\hline Galcatus. & 2 & Inde. & Erythropterus. & 2 & Ibid. \\
\hline Galeritus. & 2 & Sumatra. & Gularis. & 1 & Ibid. \\
\hline Ginginianus. & 5 & Ibid. & Nubicus. & 2 & Sumatra. \\
\hline Hydrocorax. & 2 & Philippines. & Ornatus. & 1 & Timor: \\
\hline Limbatus. & 2 & Abyssinie. & Quinticolor. & 4 & Java. \\
\hline Lunatus. & 1 & Java. & Savignii. & 2 & Afrique. \\
\hline Malabaricus. & 2 & Ibid. & Savigniioides. & 7 & Ibid. \\
\hline Nasutus. & 4 & Afrique. & Sonnini. & 2 & Ibid. \\
\hline Panayensis. & 1 & Manille. & Viridis. & 5 & Inde. \\
\hline
\end{tabular}

Plicatus.

Rhinoceros.

6 Ibid.

6 Sumatra.

Ruficollis. -

Sulcatus.

5 Célébes.

Espèce indéterminée. $11 /$ Ibid.

\section{Ggarre CEYX.}

\begin{tabular}{l|l|l} 
Azurea. & 4 & Nouv.-Hollande. \\
Madagascarionsis. & 1 & Madagascar. \\
Espéce indéterminée. & $\mathbf{3}$ &
\end{tabular}

\section{GENRE DACEO.}

Atricapilla.

Concreta.

Fusca.

Fuscicapilla.

Gaudichandi.

Monaca.

Pulchella.

Pygmeu.

Ruficeps.

Senegaloides.

Plus, 3 individus formant 2 especes indéterminées.

GENRE MEROPS.

Amirlus.

Apiraster.

Allertoni.

Badius.

\begin{tabular}{|c|l||}
2 & Inde. \\
5 & Ibid. \\
2 & Austialie. \\
2 & Afrique. \\
2 & Nouv.-Guinée. \\
1 & Célèes. \\
4 & Java. \\
4 & Afrique. \\
2 & Maurice. \\
1 & Afrique.
\end{tabular}

Duvaucelii.

Isidori.

\section{PASSEREAUX GRIMPEURS.}

\section{CENRE}

\begin{tabular}{l|c|l} 
Aurantiorufus. & 1 & Brésil. \\
Brasiliensis. & $\mathbf{2}$ & Ibid. \\
Martii. & $\mathbf{3}$ & Ibid. \\
Superciliaris. & $\mathbf{4}$ & Campêche. \\
Tulu. & $\mathbf{1}$ & Brésil.
\end{tabular}

Plus, 7 individus formant 4 espéces indéterminées ou inédites du Mexique.

\section{GENRE TODUS.}

Viridis.

| \$ |Amérique mér. 


\begin{tabular}{|c|c|c|}
\hline ESPE்CES. & 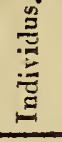 & НАВІТАТ. \\
\hline Corvinus & 2 & Inde. \\
\hline Cyanecollis. & 2 & Ibid. \\
\hline Frontalis. & 3 & Ibid. \\
\hline Grandis. & 2 & Ibid. \\
\hline Gularis. & 2 & Ibid. \\
\hline Henricii. & 2 & Ibid. \\
\hline Kotorea. & 2 & Ibid. \\
\hline Mystacophanos. & 3 & Ibid. \\
\hline Parvus. & 2 & Ibid. \\
\hline Philippensis. & 2 & Afrique. \\
\hline Pyrolophus. & 2 & Ibid. \\
\hline Rosaceicollis. & 3 & Bogota. \\
\hline $\begin{array}{l}\text { Rubrifrons. } \\
\text { Versicolor. }\end{array}$ & $\begin{array}{l}5 \\
2\end{array}$ & Afrique. \\
\hline Viridis. & 6 & \\
\hline
\end{tabular}

Gerre capito.

Collaris.

Macrorhynchus.

Maculatus.

Melanoleucus.

Melanotis.

Tamatia.

$\mid$\begin{tabular}{l|l}
$\mathbf{1}$ & Amérique. \\
$\mathbf{3}$ & Ibid. \\
$\mathbf{2}$ & Ibid. \\
$\mathbf{2}$ & Ibid. \\
$\mathbf{3}$ & Ibid. \\
$\mathbf{2}$ & Ibid.
\end{tabular}

Plus, $\mathbf{3}$ individus indéterminę́s du Mexique.

\section{GenRe CENTROPUS.}

\begin{tabular}{l|l|l|} 
Athiops. & 1 & Java. \\
Affinis. & $\mathbf{3}$ & Ibid. \\
Celebensis. & 2 & Chili.. \\
\hline Gigas. & 1 & Nouv.-Hollande. \\
Goliath. & 1 & Ibid. \\
Nigrorufus. & $\mathbf{5}$ & Afrique. \\
Philippensis. & 4 & Philippines. \\
Rufinus. & 1 & Afrique. \\
Senegalensis. & 8 & Ibid. \\
Sirkee. & 1 & Himalaya. \\
Superciliosus. & $\mathbf{7}$ & Inde.
\end{tabular}

Plus, 9 individus formant 4 espèces indéterminées ou inédites de diverses localités.

\begin{tabular}{|c|c|c|}
\hline ESPÈCES. & 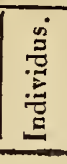 & HABITAT. \\
\hline \multicolumn{3}{|c|}{ GENRE COCCYZUS. } \\
\hline $\begin{array}{l}\text { Caruleus. } \\
\text { Cayanus. } \\
\text { Crislatus. } \\
\text { Delalandei. } \\
\text { Erythrorhynchus. } \\
\text { Flavirostris. } \\
\text { Geoffroyi. } \\
\text { Monachus. } \\
\text { Novius. } \\
\text { Reynaudii. } \\
\text { Espèce indéterminée. }\end{array}$ & $\begin{array}{l}1 \\
7 \\
2 \\
2 \\
2 \\
1 \\
1 \\
2 \\
4 \\
1 \\
2\end{array}$ & $\begin{array}{l}\text { Madagascar. } \\
\text { Brésil. } \\
\text { Madagascar. } \\
\text { Ibid. } \\
\text { Java. } \\
\text { Amérique mér. } \\
\text { Brësil. } \\
\text { Cayenne. } \\
\text { Amérique mėr. } \\
\text { Madagascar. } \\
\text { Zanzibar. }\end{array}$ \\
\hline
\end{tabular}

GENRE CROTOPHAGA.

Ani.

Casusii.

Major.

\section{GrinRE CUCU1US.}

Eneus.

Americanus.

Auratus.

Canorus.

Chalciles.

Cineraceus.

Clamosus.

Coromandus.

Cupreus.

Edolius.

Erythrophthalmus.

Flavus.

Glandarius.

Klaasii.

Levaillantii.

Lucidus.

Lugubris.

Melanoleucos.

Minor.

Orientalis.

Serratus.

Solitarius.

Sparverioides.

Sparverius.

\begin{tabular}{l|l}
$\mathbf{2}$ & Brésil \\
$\mathbf{3}$ & Ibid. \\
$\mathbf{3}$ & Ibid.
\end{tabular}

\begin{tabular}{|l|l}
$\mathbf{1}$ & Afrique. \\
$\mathbf{3}$ & Amérique mér. \\
$\mathbf{6}$ & Afrique. \\
$\mathbf{1}$ & Europe. \\
$\mathbf{4}$ & Inde. \\
$\mathbf{2}$ & Noun.-Hollande. \\
4 & Inde. \\
$\mathbf{5}$ & Afrique \\
$\mathbf{1}$ & Java. \\
$\mathbf{2}$ & Amérique sept. \\
$\mathbf{3}$ & Inde: \\
$\mathbf{4}$ & Afrique. \\
$\mathbf{3}$ & Ibid. \\
$\mathbf{2}$ & Ibid. \\
$\mathbf{4}$ & Nouv.-Hollande. \\
4 & dava. \\
4 & Afrique. \\
$\mathbf{3}$ & Cayenne. \\
$\mathbf{7}$ & Inde. \\
$\mathbf{1}$ & Afrique. \\
$\mathbf{4}$ & Ibid. \\
$\mathbf{1}$ & Himalaya. \\
$\mathbf{4}$ & Ibid. \\
&
\end{tabular}




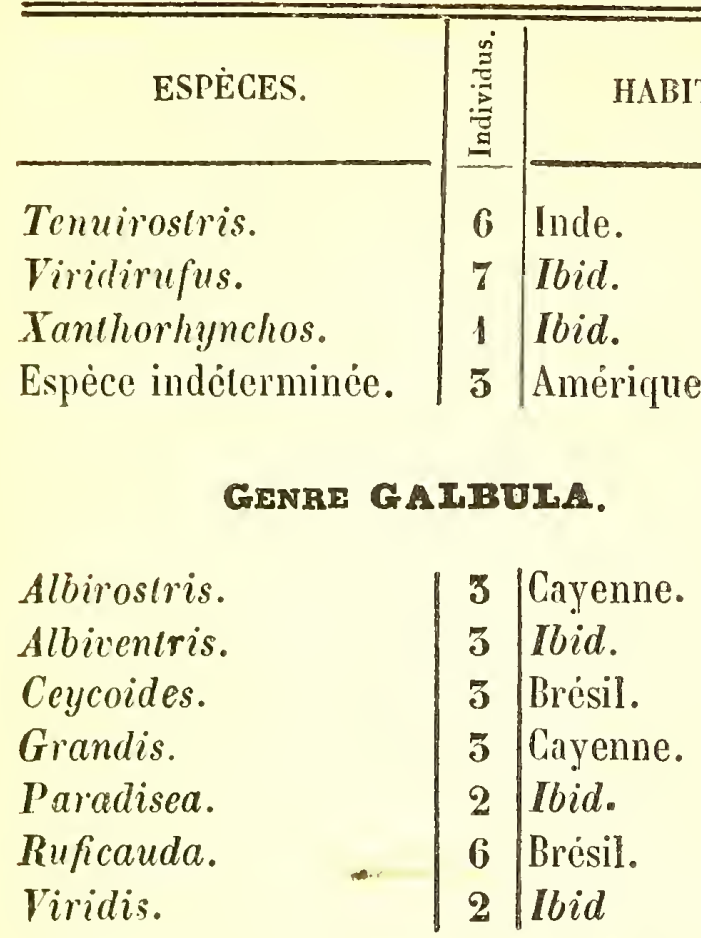

\section{GenRE INDICATOR}

\begin{tabular}{l|l|l} 
Albirostris. & / & Afrique. \\
Major. & 5 & Ibid. \\
Minor. & 2 & Ibid.
\end{tabular}

\section{GENRE LIPTOSOMUS.}

Viridis.

I. |Madagascar.

\section{GENRE ITPORRII.}

Fusca.

$\frac{\text { Phaiolencos. }}{\text { Torquala. }}$

Espece indéterminée. $\quad 2$ Colombie.

GENRE MICROPOGON.

Aureus.

Cayanensis.

Fuliginosus.

Lalhamii

Margarilatus.

Novius.

Sulphuralus.

Espece indélerminée. $\quad 2$ Bogota.

\section{GenRe monasa. \\ GenRe Montasa.}

Personala.

Phaioleucos.

T'enelorio.

Trunquilla.
3 Péron.

2 Cayenne.

5 Inde.

2 Ibid.

3 Abyssinic.

5 Brésil.

3 Arrique.

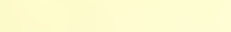

ESPÈCES.

HABITAT.

\section{GENRE IPHOEICOPHIRUS}

Calyorhynchus.

Elongalus.

Pyrrhocephalus.

Sumalranus.

Superciliosus.

Tristis.

Viridis.

Espéce indélerminée.

\begin{tabular}{|c|l|l}
2 & Inde. \\
1 & Ibid. \\
1 & Ibid. \\
3 & Ibid. \\
2 & Ibid. \\
2 & Ibid. \\
2 & Ibid. \\
1 & Ibid.
\end{tabular}

\section{GENRE PICUMNUS.}

Cirrhaius.

Exilis.

Minulissimus.

\begin{tabular}{l|l}
6 & Amérique mér. \\
4 & Ibid. \\
9 & Guiane.
\end{tabular}

Plus, 5 individus formant 2 especes indéterminées de Bogota.

\section{GERE PICU:}

Abingoni.

Adspersus.

Affinis.

Albirostris.

Arator.

Auratus.

Aarrifrons.

Aurulentus.

Awokeri.

Badioides.

Bennetii.

Biarmicus.

IBicolor.

Brachyurus.

Brunnifrons.

Campestris.

Canadensis.

Cancellatus.

Canente.

Canus.

Capensis.

Carolinus.

Cayennensis.

Chilensis.

Chlorolophus.

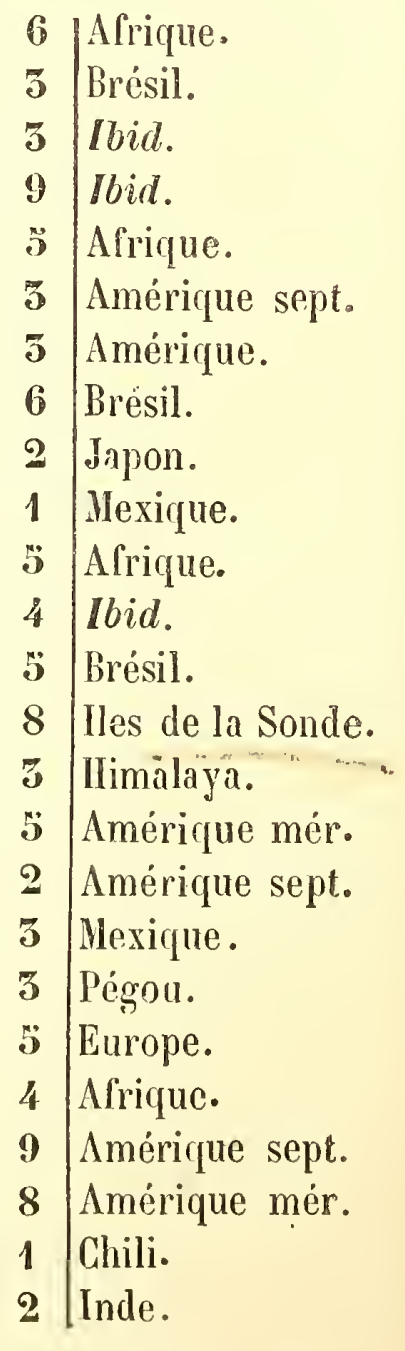




\begin{tabular}{|c|c|c|c|c|c|}
\hline ESPECES. & 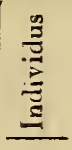 & HABITAT. & ESPĖCES. & 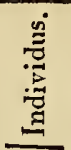 & HABITAT. \\
\hline Chlorozoslus. & 2 & Amérique. & Percussus. & 2 & Cuba. \\
\hline Cinnamomeus. & 7 & Guiane. & Pilealus. & 3 & Amérique sept. \\
\hline Concrelus. & 6 & Iles de la Sonde. & Pacilophos. & 6 & Iles de la Sonde. \\
\hline Dimidialus. & 9 & Ibid: $\quad-\ldots$ & Polyzonus. & 2 & Brésil. \\
\hline Elegans. & 5 & Mexique. & Portoricensis. & 2 & Portorico. \\
\hline Eryihrocephalus. & 6 & Amérique sept. & Principalis. & 2 & Amérique sept. \\
\hline Erylhronolus. & 2 & Ceylan. & Pubescens. & 5 & Ibid. \\
\hline Er!/lhrops. & $\breve{s}$ & Brésil. & Pulverulenlus. & 4 & Sumatra. \\
\hline Flavescens. & 7 & Ibid. & Punctuligerus. & 6 & Sénégal. \\
\hline Flavicans. & 8 & Ibid. & Puniceus. & 6 & Iles de la Sonde. \\
\hline Flavifrons. & 5 & Ibid. & Pyrrhomelas. & 4 & Inde. \\
\hline Frenalus. & $\mathfrak{5}$ & Bogata. & Regalis. & 5 & Amérique. \\
\hline Fulviscapus. & 7 & Afrique. & Rivolii. & 3 & Bogota. \\
\hline Fulvus. & 4 & Ciélèbes. & Robustus. & ఏૅ & Amérique mér. \\
\hline Galeatus. & 2 & Brésil. & Rubiginosus. & 6 & Brésil. \\
\hline Goensis. & $\breve{5}$ & Inde. & Rubricollis. & 4 & Amérique mér. \\
\hline Hamalribon. & 3 & Philippines. & Rubrifrons. & 7 & Brésil. \\
\hline Hirundinaceus. & 5 & Brésil. & Rufus. & כ้ & Guiane. \\
\hline Iclerocephalus. & 7 & Ibid: & Sanguineus. & $\breve{z}$ & Amėrique. \\
\hline Impcrialis. & 2 & Mexique. & Santacruzi. & 4 & Ibid. \\
\hline Javensis. & 3 & Java. & Scalaris. & 2 & Mexique. \\
\hline Jumana. & 1 & Brésil. & Senegalensis. & 6 & Sẻnégal. \\
\hline Larvalus. & 2 & Jamaïque. & Spilogaster. & 1 & Himalaya. \\
\hline Leucogasler. & 4 & lles de la Sonde. & Squamatus. & 3 & Inde. \\
\hline Leuconolus. & 3 & Europe. & Strialus. & 5 & Saint-Domingue. \\
\hline Lichtensleinii. & 1 & Ile de Luçon. & Subelegans. & 3 & Amérique. \\
\hline Linealus. & 12 & Amérique mẻr. & Tiga. & 9 & Iles de la Sonde \\
\hline Macei. & 8 & Iles de la Sonde. & Tinnunculus. & 1 & Cayenne. \\
\hline Magellanicus. & 2 & Amérique mér. & Torqualus. & 1 & Amérique sept. \\
\hline Mahrattensis. & $\mathbf{3}$ & Inde. & Tridaclylus. & 6 & Europe, etc. \\
\hline Major. & 12 & Europe. & Validus. & 7 & Java. \\
\hline Marginatus. & 5 & Java. & Variegatus. & 7 & Manille. \\
\hline Marlius. & 4 & Europe. & Varius. & 10 & Amérique sept. \\
\hline Medius. & 7 & Europe. & Villosus. & 7 & Ibid. \\
\hline Melanochlorus. & 6 & Amérique mér. & Viridis. & 8 & Europe, etc. \\
\hline Melanopogon. & 7 & Cuba. & Wagleri. & 4 & Amérique mér. \\
\hline Mentalis. & $\check{\jmath}$ & Java. & \multirow{5}{*}{\multicolumn{3}{|c|}{$\begin{array}{l}\text { Plus, } 49 \text { individus formant } 18 \text { espéces indé- } \\
\text { terminées ou inèdites provenant de diverses } \\
\text { localités. }\end{array}$}} \\
\hline Mexicanus. & 7 & Mexique. & & & \\
\hline Miniatus. & $\breve{3}$ & Iles de la Sonde. & & & \\
\hline Minor. & 8 & Europe. & & & \\
\hline Mullicolor. & 3 & Guiane. & & & \\
\hline Nubicus. & 7 & Afrique. & \multirow{2}{*}{\multicolumn{3}{|c|}{ GENRE POGOIIAS. }} \\
\hline Obsolelus. & 4 & Ibid. & & & \\
\hline Occipitális. & 2 & Inde. & Brucei. & 1 & A frique. \\
\hline Passerinus. & 10 & Amérique mér. & $\|$ Lavirostris. & 1 & Ibid. \\
\hline
\end{tabular}




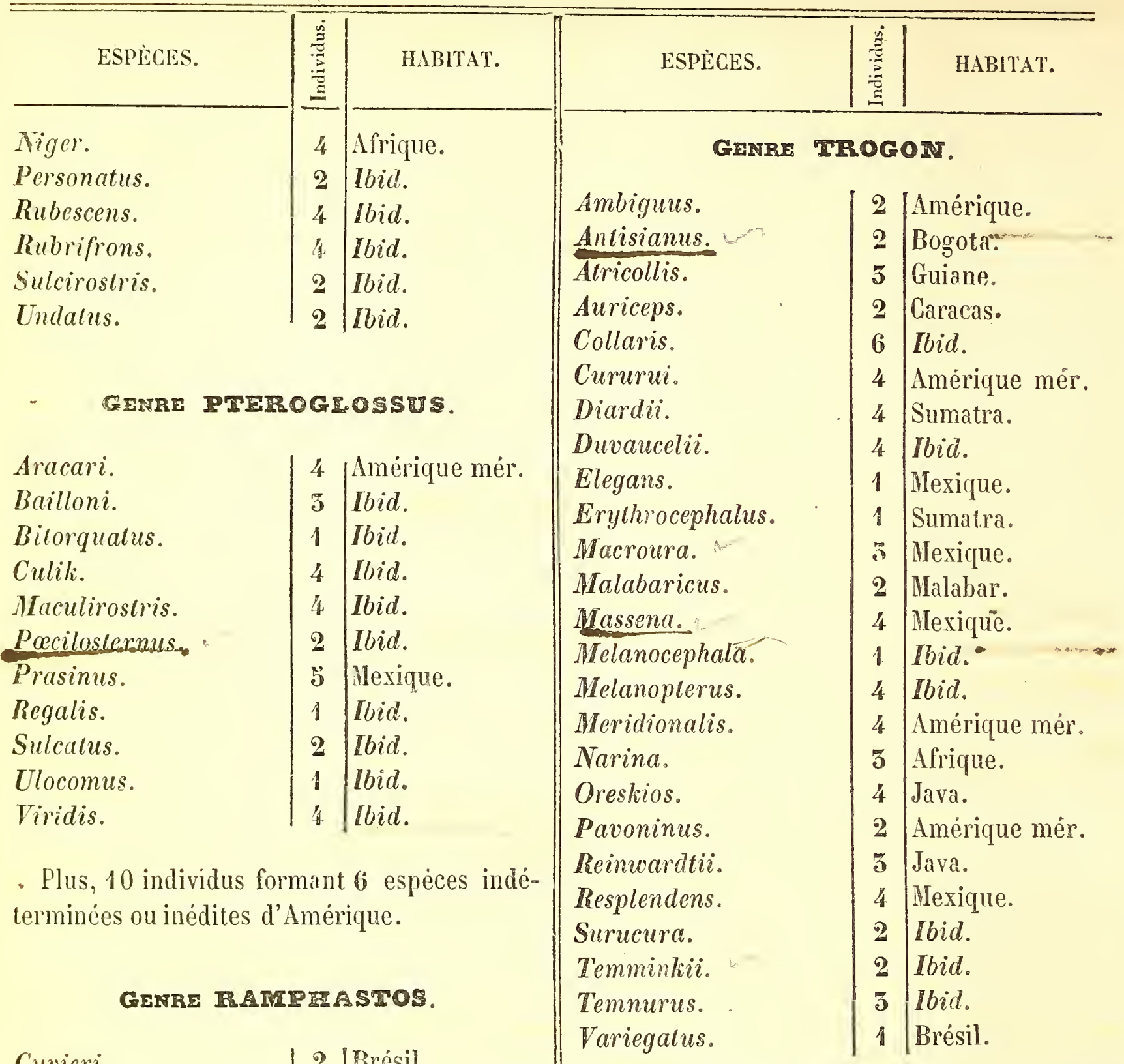

Cuvieri.

Dicoloras.

Erylhroriynchus.

Swainsonii.

Vilellinus.

Plus, 9 individus formant 4 espéces indétermineses ou inédites d'Amerrique.

\section{GewRe SAUROTHERA.}

Bollac.

Californiana.

Velula.

Espece indriternince.

\begin{tabular}{l|l}
2 & Galifornie. \\
1 & Ibid. \\
2 & Guiane. \\
2 & Amerique.
\end{tabular}

Plus, 10 individus formant 7 espèces indéterminées ou inédites de diverses localités.

\section{GERRI TURACUS.}

Concolor.

Corythaix.

Giganteus.

Leucogaster.

Leucotis.

\section{Paulina.}

Porphyreolophus.

Variegalus.

Violaceus.

Zonurus.

\begin{tabular}{|l|l}
4 & Afrique. \\
2 & Ibid. \\
1 & Ibid. \\
1 & Abyssinie. \\
2 & Ibid. \\
1 & Afrique. \\
2 & Ibid. \\
2 & Ibid. \\
2 & Ibid. \\
1 & Aloyssinie.
\end{tabular}

\section{GENRE YUINX.}

Pecloralis.

Torquilla. $\mid$\begin{tabular}{l|l}
2 & Afrique. \\
$\mathbf{3}$ & Europe, etc.
\end{tabular} 


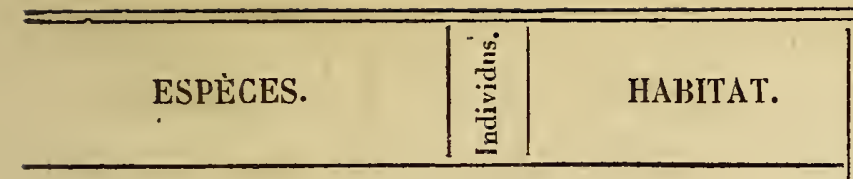

GALLINACÉS.

GENRE ATTAGIS.

Lalreillii.

| 1 |Chili.

\section{GenRe Cotumba.}

Abyssinica.

Enas.

Afra.

Albitorques.

Armillaris.

Aromatica.

Arquatrix.

Aurila.

Aurivora.

Australis. -

Aymara. n

Bengalensis.

Bilorquala.

Boliviana.

Calva.

Cambayensis.

Capellei.

Capensis.

Capistrata.

Carolinerasis.

Carunculata.

Chalcoplera.

Cinerea.

Coronata.

Cruenta.

Cyanocephala.

Denisea.

Diademala.

Dilopha.

Elegans.

Erylhroptera.

Ewingii.

Forsteri.

Frontalis.

Gelastis.

Geoffroyii.

Guinea.

Gularis.
5 Afrique.

2 Europe.

2 Afrique.

2 Ibid.

3 Inde.

3 Java.

4 Afrique.

1 Martinique.

2 Canaries.

3 Madagascar.

1 Amérique mér.

1 Bengale.

4 Inde.

2 Chili.

4 Afrique.

3 Ibid.

2 Inde.

4 Afrique.

4 Inde.

9 Amérique.

2 Inde.

4 Nouv.-Ilollande.

6 Brésil.

1 Nouv.-Guinée.

3 Philippines.

3 Jamaïque.

2 Colombie.

4 Iles Mariannes.

2 Nouv.-Hollande.

3 Ibid.

4 Coupang.

5 Iles Mariannes.

1 Célẻbes.

5 Guiane.

2 Japon.

3 Brésil.

3 Afrique.

1 Célébes.
Humilis.

Jambu.

Javanica.

Lacernulata.

Larvata.

Leptogrammica.

Leucocephala.

Leucomela.

Leuconota.

Leucoplera.

Leucolis.

Levaillantii.

Litloralis.

Livia.

Loculrix.

Lophotes.

Luciuosa.

Luzeus.

Macquari.

Maculosa.

Madagascariensis.

Magnifica.

Malaccensis.

Manadensis. W

Martinica.

Maugeus.

Melanocephala.

Migraloria.

Mililaris.

Minula.

Modesla.

Nicobarica.

Olax.

Oxyura.

Pagodus.

Palumba.

Pampusa.

Passerina.

Paulina. 2

Perspicillata.

Phasianella.

Piclurala.

Porphyrea.

Porloricensis.
HABITAT.

Bengale.

4 Sumatra.

7 Inde.

2 Ibid.

3 Afrique.

1 Java.

1 Jamaïque.

2 Nouv.-Hollande.

Limalaya.

2 Brésil.

2 Manille.

3 Afrique.

1 Moluques.

2 Europe, etc.

4 Brésil.

1 Nouv.-Hollande.

3 Ibid.

1 Abyssinie.

3 Nouv.-Hollande.

2 Amérique mér.

2 Maclagascar.

2 Nouv.-Hollande.

2 Moluques.

2 ibid.

8 Mexique.

5 Coupang.

๖ Java.

3 Amérique sept.

4 Inde.

5 Brésil.

1 Inde.

2 Moluques.

5 Inde.

1 Ibid.

1 Ibid.

2 Europe.

1 Guiane.

4 Amérique.

2 Célébes.

3 Philippines.

6 Ibid.

2 Ile de France.

1 Inde.

4 Iles de.la Sonde.

1 Porto-Rico. 


\begin{tabular}{|c|c|c|}
\hline ESPECES. & 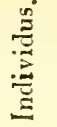 & HABITAT. \\
\hline Psiltacea. & 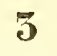 & Timor. \\
\hline Puella. & 1 & Nouv.-Irlande. \\
\hline Purpurata. & 7 & Guam. \\
\hline Risoria. & 2 & Afrique. \\
\hline Rivolii. & 1 & Inde. \\
\hline Rosacea. & 1 & Ibid. \\
\hline Rubricapilla. & 5 & lbid. \\
\hline Ruficeps. & 2 & Ibid. \\
\hline Rufina. & 4 & Guiane. \\
\hline Scripla. & 2 & Nouv.-Hollande. \\
\hline Sieboldii. & 2 & Japon. \\
\hline Spadicea. & 2 & Iles des Amis. \\
\hline Speciosa. & 己゙ & Cayenne. \\
\hline Squamosa. & 2 & Brésil. \\
\hline Strepitans. & 4 & Ibid. \\
\hline Talpacoli. & 4 & Ibid. \\
\hline Tigrina. & 4 & Inde. \\
\hline Turlur. & 1 & Europe. \\
\hline Tympanistria. & 4 & Afrique. \\
\hline Vernans. & $\breve{s}$ & Java. .... \\
\hline Violacea. & 2 & Amérique mér. \\
\hline Viridis. & 5 & Bourou. \\
\hline Viridissima. & 4 & Inde. \\
\hline
\end{tabular}

Plus, $\mathbf{5 6}$ individus formant 17 espèces indèterminées ou inédites provenant de diverses localités.

\section{GENRE COTIRINIR.}

Australis.

Coturnix.

Erythrorhyncha.

Excalfactoria.

Pectoralis.

Textilis.

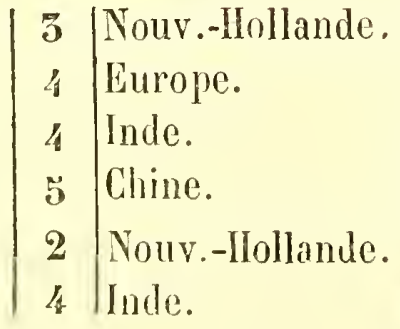

GenRe CRAK.

Aleclor.

Carunculatı.

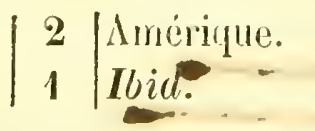

GenRe CRTRTONIX.

Coronalus.

Espere indrentunes.
ESPE்CES.

\section{GERRE EUILCOMUS.}

\begin{tabular}{l|l|l} 
Erylhrophthalmus. & 2 & Inde. \\
Ignilus. & 2 & Ibid.
\end{tabular}

\section{GENRE FRARTOLINUS.}

Cruentus.

Natalensis.

Nivosus.

Perlala.

Ponlicerianus.

Spadicea.

Swainsonii.

\begin{tabular}{|l|l}
1 & Inde. \\
4 & Afrique. \\
2 & Inde. \\
$\mathbf{5}$ & Chine. \\
4 & Inde. \\
4 & Madagascar. \\
2 & Afrique.
\end{tabular}

\section{CEARE GREIUS.}

Eneus.

Bankiva.

Furcatus.

Sonnerali.

Espèce indéterminée. 1 IIimalaya.

\section{GERE EEMIPODIUS.}

\section{Hotlen!otus.}

Meiffrenii.

Nigricollis.

Pugnax.

Strialus.

Tachydromus.

Thoracicus.

\begin{tabular}{l|c|l} 
Varius. & 5 & Nouv \\
Espéce indéterminée. & 1 & Inde.
\end{tabular}

3 Afrique.

2 Ibid.

1 Madagascar.

6 Java.

1 Ile de Lucon.

4 Europe.

8 Philippines.

5 Nouv.-Hollande.

\section{GERE IOPEOREORES.}

Refulexiins

Espéce indélerminèe. 1 Ibid.

\section{GenRz ITETEAGRIS.}

Gallo-pavo.

1 | Amérique sept.

\section{GERRE NUMIDA.}

Meleagris.

Mitrala.

Plylorhyncha.
2 Afrique.

2 Ibid. 


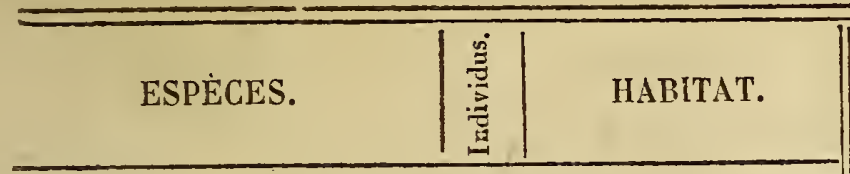

GENRE OPISTHOCOMUS.

Crislatus.

I 2 |Cayenne.

\section{GENRE OETIX.}

Borealis.

Californicus.

Crislala.

Elegans.

Guianensis.

Massena.

Squamalus.

\begin{tabular}{l|l}
6 & Amérique sept. \\
4 & Californie. \\
7 & Amérique. \\
2 & Ibid. \\
1 & Ibid. \\
1 & Mlexique. \\
2 & Ibid.
\end{tabular}

Plus, 3 individus formant 2 espèces de Bogota.

\section{GENRE OURAX.}

Globicera.

Mitu.

$\mid$\begin{tabular}{l|l}
1 & Colombie. \\
2 & Brésil.
\end{tabular}

gente pavo.

Cristatus.

Muticus.

$$
\mid \begin{array}{l|l}
2 & \text { Europe. } \\
2 & \text { Sumatra. }
\end{array}
$$

\section{GENRE PEIELOPE.}

Marail.

Parralioua.

Pipile.

\begin{tabular}{l|l}
3 & Amérique mér. \\
$\mathbf{3}$ & Ibid. \\
2 & Ibid.
\end{tabular}

Plus, 6 individus formant 4 espèces indéterminées ou inédites provenant d'Amérique.

\section{GenRs Pardix.}

Adansonii.

Afra.

Cambayensis.

Chulkar.

Cinerea.

Clamalor.

Clappertonii.

Dentata.

Erckelii.

Francolinus.

Fusca.

\begin{tabular}{c|l}
2 & Afrique. \\
5 & Ibid. \\
6 & Ibid. \\
1 & Amérique. \\
10 & Europe. \\
3 & Afrique. \\
2 & Abyssinie. \\
1 & Amérique mér. \\
1 & Abyssinie. \\
5 & Europe, etc. \\
7 & Afrique.
\end{tabular}

Guianensis.

Gularis.

Heyi.

Javanica .

Levaillantoides.

Megapodia.

Nudicollis.

Oculea.

Pelrosa.

Rubra.

Rubricollis.

Saxalilis.

Sephaena.

Sonninii.

Texlilis.

Thoracica.

Vaillantii.
HABITAT.

Amérique.

2 Inde.

Ibid.

2 Ibid.

2 Afrique.

3 Bengale.

4 Afrique.

3 Inde.

2 Europe.

Ibid.

5 Abyssinie.

6 Europe.

3 Afrique.

2 Amérique.

4 Inde.

1 Ibid.

2 Afrique.

Plus, $\mathbf{3}$ individus formant $\mathbf{2}$ espèces indéterminées de l'Inde.

\section{GenRe pHASIANUS.}

Albocrislatus.

Argus.

Colchicus.

Nyclhemerus.

Pictus.

Soemmeringii.

Slacei.

Torquatus.

Veneralus.

Versicolor.

\begin{tabular}{|l|l|}
$\mathbf{3}$ & Inde. \\
2 & Ibid. \\
1 & Europe. \\
2 & Chine. \\
$\mathbf{2}$ & Ibid. \\
$\mathbf{2}$ & Japon. \\
4 & Inde \\
1 & Europe. \\
1 & Chine. \\
$\mathbf{3}$ & Japon.
\end{tabular}

\section{GRNR POIYPIECTRON.}

Bicalcaralum.

Chinquis.

Emphanum.

\begin{tabular}{l|l}
3 & Malaca. \\
1 & Chine. \\
1 & Sumatra.
\end{tabular}

\section{GENRE PTEROCLES.}

Arenarius.

Bicinclus.

Coronatus.

Exustus.

Gultalus. 


\begin{tabular}{|c|c|c|}
\hline ESPECES. & 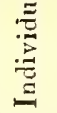 & HABITAT. \\
\hline Gutluralis. & 5 & Afrique. \\
\hline Quadricinclus. & 7 & Ibid. \\
\hline Setarius. & 6 & Europe. \\
\hline Tachypeles. & $\xi$ & Afrique. \\
\hline Variegalus. & 4 & Ibid. \\
\hline
\end{tabular}

GENRE TERAO.

Bonasia.

Canadensis.

Cupido.

Lagopus.

Medius.

Nigellii.

Phasianellus.

Salicetii.

Scolicus.

Umbellus.

Urogallus.

Telrix.

Espèce indéterminée.

\begin{tabular}{|c|l}
5 & Europe. \\
5 & Amérique sept. \\
5 & Ibid. \\
13 & Europe. \\
2 & Ibid. \\
1 & Inde. \\
1 & Amérique sept. \\
4 & Europe. \\
2 & Ibid. \\
4 & Amérique sept. \\
4 & Europe. \\
2 & Canada. \\
2 & Amérique.
\end{tabular}

CENRE PINAMUS.

Adspersus.

Boraguira.

Brasiliensis.

Cinereus.

Major.

Medius.

Noclivagus.

Obsolelus.

Rufescens.

Talaupa.

Variegalus.

Plus, 14 individus formant 10 espèces indéterminées ou inédites provenant de diverses localites.

\section{GENRE TINOCHORUS.}

Eschshollzii.

Swainsonii.

$$
\mid \begin{array}{l|l}
4 & \text { Chili. } \\
1 & \text { Ibid. }
\end{array}
$$

\section{Genre tragopan.}

IIaslingsii.

Pucrasia.

sialyrus.

\begin{tabular}{l|l}
2 & Inde. \\
3 & Ibid. \\
2 & Ibid.
\end{tabular}

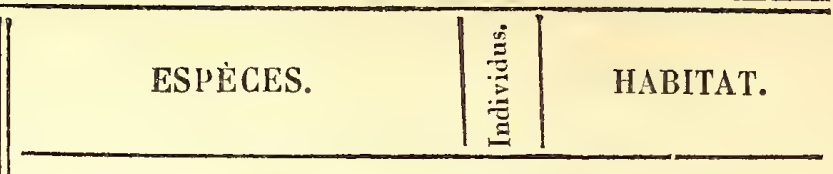

ÉCHASSIERS BRÉVIPENNES.

GIRTE APTIRIX.

Australis.

| 1 |Nouv.-Zélande.

\section{GENRE CASUARIUS.}

Australis (junior). $\quad \mid 1$ |Nouv.-Ilollande.

\section{GERRE STRETHIO.}

Rhea.

| 1 |Brésil.

\section{ÉCHASSIERS PRESSIROSTRES.}

\section{GENRE CHARADIIUS.}

Armatus.

Azarai.

Bicinclus.

Bicolor.

Bilobus.

Cantianus.

Cayanus.

Chalcoplerus.

Cinclus.

Columbinus.

Coronalus.

Crislalus.

Gallinaceus.

Goensis.

Hialicula.

Indicus.

Lampronolus.

Leucurus.

Marmoratus.

Melanogaster.

Melanoplerus.

Minor.

Monachus.

Morinellus.

Nigrifrons.

\begin{tabular}{l|l}
$\mathbf{3}$ & Afrique. \\
2 & Brésil. \\
2 & Nouv.-Hollande. \\
$\mathbf{1}$ & Bengale. \\
$\mathbf{3}$ & Inde. \\
2 & Europe. \\
2 & Cayenne. \\
1 & Sénégal. \\
$\mathbf{2}$ & Nouv.-Hollande. \\
$\mathbf{6}$ & Inde. \\
1 & Afrique. \\
4 & Europe. \\
$\mathbf{2}$ & Nouv.-Hollande. \\
$\mathbf{2}$ & Ibid. \\
$\mathbf{3}$ & Europe. \\
$\mathbf{3}$ & Inde. \\
$\mathbf{3}$ & Amérique mér. \\
$\mathbf{1}$ & Afrique. \\
1 & Amérique mér. \\
$\mathbf{9}$ & Europe, etc. \\
$\mathbf{1}$ & Afrique. \\
$\mathbf{2}$ & Europe. \\
$\mathbf{3}$ & Nouv.-Ilollande. \\
$\mathbf{2}$ & Europe. \\
$\mathbf{3}$ & Nouv.-Hollande. \\
\end{tabular}




\begin{tabular}{|c|c|c|}
\hline ESPĖCES. & 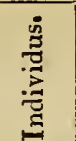 & HABITAT. \\
\hline Pecuarius. & 2 & Afrique. \\
\hline Pluvialis. & $\mathbf{3}$ & Europe. \\
\hline Pyrrhothoraxe & 3 & Europe, etc. \\
\hline Ruficollis. & 1 & Ibid. \\
\hline Sanguineus. & $\mathbf{3}$ & Afrique. \\
\hline Senegalensis. & 2 & Ibid. \\
\hline Spinosus. & $\mathbf{3}$ & Ibid. \\
\hline Vociferus. & 3 & Amérique sep \\
\hline
\end{tabular}

Plus, 18 individus formant 12 espèces indéterminées ou inédites provenant de diverses localités.

\section{GenRe CURsorius.}

Asiaticus.

Bicinctus.

Isabellinus.

Melanocephalus.

Temminkii.

$\mid$\begin{tabular}{r|l}
2 & Inde. \\
1 & Afrique. \\
4 & Europe, etc. \\
4 & Afrique. \\
1 & Abyssinie.
\end{tabular}

GenRe DIChorophus.

Cristalus.

11 |Amérique.

GENRE HTMATOPUS.

Niger.

Ostralegus.

Palliatus.

Espèce indéterminée. 11 Nouv.-Zélande.

\section{GENRE CDICNEMUS.}

Crepitans.

Longipes.

Maculosus.

Magnirostris.

Espèce indéterminée. 1 Amérique.

Espèce indéterminée. $[\mathbf{3}$ Afrique.

\section{GENRE OTIS.}

Afra.

Afroides.

Arabs.

Aurila.

Bengalensis.

\begin{tabular}{|l|l}
6 & Afrique. \\
2 & Ibid. \\
1 & Ibid. \\
4 & Inde. \\
2 & Ibid.
\end{tabular}

\begin{tabular}{|c|c|c|}
\hline ESPĖCES. & 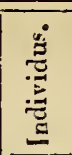 & HABITAT. \\
\hline Carulescens. & 1 & Inde. \\
\hline Denhami. & 3 & Afrique. \\
\hline Houbara. & 1 & Europe. \\
\hline Kori. & 1 & Abyssinie. \\
\hline Ludwigii. & 4 & Afrique. \\
\hline Melanogaster. & 2 & Ibid. \\
\hline Nova-Hollandia. & 1 & Nouv.-Hollande. \\
\hline Ruficrista. & 1 & Afrique. \\
\hline Scolopacea. & 3 & Ibid. \\
\hline Tarda. & 3 & Europe. \\
\hline Tetrax. & 4 & Ibid. \\
\hline Torqueola. & 4 & Afrique. \\
\hline
\end{tabular}

\section{ÉGHASSIERS GULTRIROSTRES.}

GeNRE AIVASTOMUS.

\begin{tabular}{l|l|l} 
Albus. & $\mathbf{2}$ & Inde. \\
Lamelligerus. & $\mathbf{5}$ & Afrique.
\end{tabular}

\section{GENRE ARDEA.}

Agami.

Alba.

Atricollis.

Carulescens.

Caledonica.

Callocephala.

Candidissima.

Cinerea.

Cinnamomea.

Cocoi.

Egretta.

Egrettoides.

Erythromelas.

Exilis.

Flavicollis.

Garzetla.

Golial.

Gularis.

Gulturalis.

Herodias.

Intermedia.

Jugularis.

\begin{tabular}{l|l}
4 & Cayenne. \\
$\mathbf{3}$ & Europe. \\
6 & Afrique. \\
5 & Amérique. \\
4 & Nouv.-Hollande. \\
4 & Amérique. \\
2 & Ibid. \\
5 & Europe. \\
5 & Java. \\
$\mathbf{3}$ & Brésil. \\
8 & Europe, etc. \\
$\mathbf{2}$ & Japon. \\
$\mathbf{2}$ & Amérique. \\
$\mathbf{2}$ & Java. \\
4 & Nouv.-Hollande. \\
$\mathbf{3}$ & Europe. \\
1 & Afrique. \\
$\mathbf{1}$ & Ibid. \\
$\mathbf{1}$ & Ibid. \\
$\mathbf{2}$ & Amérique sept. \\
4 & Amérique. \\
$\mathbf{2}$ & Nouv.-Hollande.
\end{tabular}




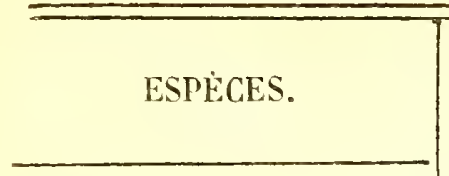

Lentiginosa.

Leucogaster.

Leucops.

Ludoviciana.

Minuta.

Nyclicorax.

Pacifica.

Ealedonica.

Philippensis.

Pileata.

Purpurea.

Pusilla.

Ralloides.

Rufescens.

Russata.

Scapularis.

Sibilatrix.

Sinensis

Soco.

Sparmannii.

Speciosa.

Stcllaris.

Sturmii.

Tigrina.

lerany.

Virescens.

Plus, 14 individus formant $\bar{z}$ espèces indé terminées ou inédites de diverses localités.

\section{GenRe CANCROMA.}

Cochlearia.

5 LAmérique.

\section{GENRE CICONIA.}

Abdimii.

Alba.

Argalu.

Australis.

Capillata.

Ephippiorhyncha.

Leucocrphala.

Leucoptera.

Maguari.

Mycteria.

Nigra.

Espece indéterminéc.

HABITAT.
ESPĖCES.

HABITAT.

GLNRE DROMAS.

Ardeola.

12 |Bengale.

\section{GENRE IETIRTGA.}

Helias.

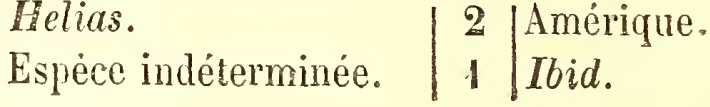

\section{GENRE GRUS.}

Antigone.

Carunculaia.

Cinerea.

Leucauchen.

Leucogeranos.

Monacha.

Paradisea.

Pavonina.

Virgo.

\begin{tabular}{|l|l}
1 & Inde. \\
2 & Afrique. \\
5 & Europe. \\
1 & Japon. \\
1 & Europe. \\
1 & Inde. \\
$\mathbf{5}$ & Afrique. \\
3 & Ibid. \\
1 & Ibid.
\end{tabular}

GENRE NOTEERODIUS.

Guarauna.

I 2 |Amérique mér.

\section{GERRE PIATAIEA.}

Ajaja.

Leucorodia. Regia.

$$
\mid \begin{array}{l|l}
4 & \text { Amérique mér. } \\
5 & \text { Europe. } \\
1 & \text { Nouv.-Hollande. }
\end{array}
$$

\section{GENRE PSOPHIA.}

Crepitans.

12 |Amérique.

\section{GENRE SCOPUS.}

Uimbrelta.

15 Afrique.

\section{GenRe a a RTALUS.}

Lacteus.

Leucocephalus.

Loculator.

Rhodinopterus. 


ESPECES. $\mid$\begin{tabular}{c|c}
$\sum_{0}^{0}$ \\
\hline
\end{tabular}

ÉCHASSIERS LONGIROSTRES.

GeNRE CAIIdRIS.

Arenaria.

| 7 |Europe.

\section{GENRE HIMANTOPUS.}

Candidus.

Leucocephalus.

Melanaucon.

Melanopterus.

\begin{tabular}{|l|l|}
2 & Afrique. \\
2 & Nouv.-Hollande. \\
1 & Timor. \\
4 & Europe.
\end{tabular}

Plus, 6 individus formant 2 espèces indéterminées d'Amérique.

\section{Genre IRIS.}

\begin{tabular}{l|l|l|} 
Alba. & 1 & Amėrique. \\
Calva. & 1 & Afrique. \\
Carunculata. & 1 & Ibid. \\
Cayennensis. & 3 & Cayenne. \\
Cristala. & 1 & Madagascar. \\
Falcinellus. & 2 & Europe. \\
Hagedasch. & 4 & Afrique. \\
Lamellicollis. & 1 & Nouv.-Hollande. \\
Leucon. & 5 & Java. \\
Melanopsis. & $\mathbf{3}$ & Amérique mér. \\
Nudifrons. & 1 & Brésil. \\
Papiliosa. & 1 & Afrique. \\
Plumbeus. & 1 & Amérique mér. \\
Religiosa. & 5 & Afrique. \\
Rubra. & $\mathbf{3}$ & Amérique.
\end{tabular}

Plus, 8 individus formant 4 espèces indéterminées ou inédites de diverses localités.

\section{Genre imimosa.}

Fedoa.

Hudsonica.

Melanura.

Meyeri.

Rufa.

Terek.

\begin{tabular}{|l|l}
1 & Amérique sept. \\
$\mathbf{3}$ & Chiloé. \\
4 & Europe, etc. \\
1 & Ibid. \\
4 & Ibid. \\
2 & Ibid.
\end{tabular}

Plus, 13 individus formant 9 espéces indéterminées ou inedites de diverses localités.
ESPÈCES.

HABITAT.
GenRe NumeiniUs.

Arquata.

Brevirostris.

Longirostris.

Pheopus.

Ienuirostris.

Plus, 2 individus formant 2 espèces indéterminées de Bogota.

GENRE PHATAROPUS.

Hyperboreus.

Platyrhynchus.

Wilsonii.

$$
\mid \begin{array}{l|l}
1 & \text { Europe. } \\
5 & \text { Ibid. } \\
6 & \text { Amérique sept. }
\end{array}
$$

\section{GENRE RECURVIBOSTRA.}

Avocetla.

Rubricollis.

$\mid$\begin{tabular}{l|l}
1 & Europe. \\
1 & Inde.
\end{tabular}

\section{GenRe RTITITCEAEA.}

Capensis.

Hilaira.

\begin{tabular}{|l|l}
3 & Java. \\
1 & Brésil.
\end{tabular}

\section{Gente scolopax.}

Equatorialis.

Gallinago.

Gallinula.

Gigantea.

Grisea.

Major.

Minor.

Rusticola.

Wilsonii.

\begin{tabular}{|l|l}
1 & Abyssinie. \\
4 & Europe. \\
2 & Ibid. \\
1 & Brésil. \\
2 & Europe. \\
2 & Ibid. \\
2 & Amérique sept. \\
4 & Europe. \\
1 & Amérique sept.
\end{tabular}

Plus, 4 individus formant $\mathbf{3}$ espèces indéterminées de diverses localités.

\section{GenRE STRERSTAS.}

Collaris.

Townsendi. $\mid$\begin{tabular}{l|l}
8 & Europe. \\
1 & Amérique sept.
\end{tabular}

\section{GENR TOTANUף.}

Bartramia.

Brevipes.

Calidris.

Flavipes.
2 Ibid.

\begin{tabular}{l|l}
$\mathbf{2}$ & Europe. \\
$\mathbf{3}$ & Amérique sept. \\
$\mathbf{2}$ & Amérique. \\
$\mathbf{3}$ & Europe. \\
$\mathbf{2}$ & Ibid.
\end{tabular}




\begin{tabular}{|c|c|c|}
\hline ESpÈCES. & 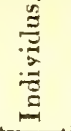 & HABITAT. \\
\hline Fuscus. & 8 & Inde. \\
\hline Glareola. & 5 & Europe. \\
\hline Glotis. & 4 & Ibid. \\
\hline Hypoleucos. & 4 & Ibid. \\
\hline Macularia. & 2 & lbid. \\
\hline Ochropus. & 5 & Ibid. \\
\hline Semipalmatus. & 3 & Toid: \\
\hline Solitarius. & $\ddot{z}$ & Brésil. \\
\hline Stagnatilis. & 2 & Europe. \\
\hline
\end{tabular}
terminées ou inédites provenant de diverses localités.

\section{GENRE TRINGA.}

Cinerea.

Himanthopus.

Marilima.

Minuta.

Pectoralis.

Pugnax.

Subarquala.

Temminkii.

Variabilis.

Plus, 7 individus formant $ّ$ espèces indéterminées ou inédiles provenant de diverses localités.

\section{EGHASSIERS MACRODACTYLES.}

\section{GeirRe CHIOJNIS.}

Minor.

Vaginalis.

$\mid$\begin{tabular}{l|l}
1 & Nouv.-Ilollande. \\
1 & Ibid.
\end{tabular}

GENRE FULICA.

Alra.

Cristata.

$$
\mid \begin{array}{l|l}
9 & \text { Europe. } \\
2 & \text { Madagascar. }
\end{array}
$$

Getre Gatuinuta.

Baillomii.

Carolina.

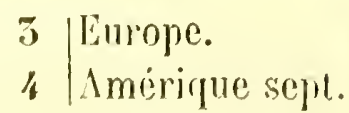

Cayanensis.

Chloropus.

Crex.

Elegans.

Eurizona.

Flavirostris.

Lateralis.

Lugubris.

Melanops.

Mystacina.

Nevia.

Nigra.

Phonicura.

Poliolis.

Porzana.

Pusilla.

Rufa.

\begin{tabular}{|c|l}
\hline 2 & \multicolumn{1}{|c}{ Habitat. } \\
\hline 2 & Guiane. \\
\hline 2 & Europe. \\
2 & Ibid. \\
2 & Amérique sept. \\
2 & Java. \\
1 & Cayenne. \\
3 & Brésil. \\
1 & Inde. \\
5 & Amérique sept. \\
2 & Java. \\
2 & Ibid. \\
6 & Afrique. \\
$\mathbf{3}$ & Ceylan. \\
1 & Guiane. \\
4 & Europe, elc. \\
1 & Ibid. \\
4 & Amérique sept.
\end{tabular}

Plus, 14 individus formant 12 espèces indéterminées ou inédites de diverses localités.

\section{GinRe CTABTOLA.}

Laclea.

Ocularis.

Torquala.

Espéce indéterminée. 1 Java.

\section{GENRE EGACEPHALON.}

Maleo.

1 |Célèbes.

\section{GENRE MEGATODIUS.}

Freycineti.

Thubripes.

$\mid$\begin{tabular}{l|l}
1 & Nouv.-Guinẻe. \\
1 & Célèbes.
\end{tabular}

GENRE PARPA.

Anea.

A/ricana.

Gallinacea.

Jacana.

Sinensis.

$$
\begin{array}{l|l}
3 & \text { Java. } \\
1 & \text { Afrique. } \\
2 & \text { Célébes. } \\
5 & \text { Amérique mér. } \\
2 & \text { Bengale. }
\end{array}
$$

Plus, 8 individus formant 5 espéces indéterminées on inédites de diverses localités. 


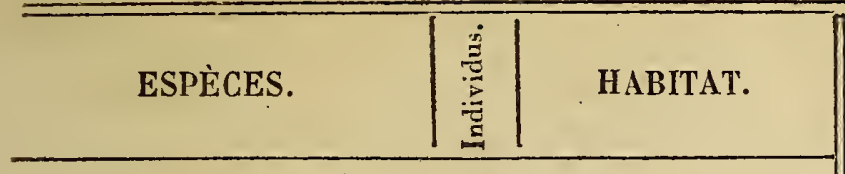

GENRE PAIA MEDEA.

Chavaria.

Cornuta.

Espèce indéterminée. $\quad{ }_{1}$ I Ibid.

\section{GenRe PECHICOPt ERUS.}

Antíquorum.

Ignipalliatus.

Minor.

Ruber.

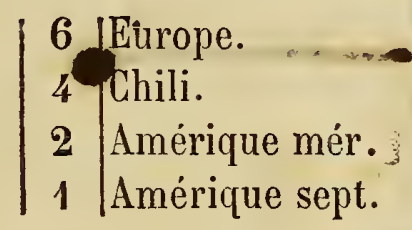

\section{GENRE PORPHYRIO.}

Hyacinthinus.

Marlinica.

Melanolus.

Smaragdinus.

Smaragnotus.

Plus, 3 individus formant 2 espèces indéterminées de Bahia.

\section{GenRe RaIIUS.}

Aquaticus.

Bicolor.

Carulescens.

Cayanensis.

Celebensis.

Exilis.

Fuscus.

Gigas.

Gularis

Longirostris.

Madagascariensis.

Mangle.

Nigricans.

Philippensis.

Saracura.

Torqualus.

Variegatus.

Virginianus.

Plus, 10 individus formant 7 espèces indéterminées ou inédites provenant de diverses localitès.
ESPÈCES.

HABITAT.

Ganke Tategalius.

Cuvieri. 11 |Nouv.-Guinée.

\section{PALMIPÈDES BRACHYPTÈRES.}

\section{Grine ALCA.}

Brunnichii.

Impennis.

Torda.

$\mid$\begin{tabular}{l|l}
2 & Europe. \\
1 & Ibid. \\
4 & Ibid.
\end{tabular}

GENRE APTENODTTES.

Patagonica.

I 3 |Malouines.

GENRE CATARREACTES.

Chrysocoma.

$\mid \mathbf{3}$ |Malouines.

\section{GENRE COIYMBUS.}

Arcticus.

Glacialis.

Septentrionalis.

\begin{tabular}{|l|l}
4 & Europe. \\
4 & Ibid. \\
4 & Ibid.
\end{tabular}

\section{GENRE HELIORNIS.}

Senegalensis.

Surinamensis.

\begin{tabular}{l|l}
2 & Afrique. \\
4 & Guiane.
\end{tabular}

\section{GENRE MORMON.}

Cirrhatus.

Fratercula.

Glacialis.

$$
\mid \begin{array}{l|l}
1 & \text { Mers boréales. } \\
4 & \text { Europe. } \\
\mathbf{3} & \text { Ibid. }
\end{array}
$$

\section{GEMRE PHALERIS.}

Cristatella.

Superciliata.

$$
\mid \begin{array}{l|l}
1 & \text { Japon. } \\
1 & \text { Amérique sept. }
\end{array}
$$

\section{GHNRE PODICEPS.}

Auritus.

Calypareius.

Carolinensis.

Cornutus.

$$
\begin{array}{|l|l}
5 & \text { Europe. } \\
2 & \text { Malouines. } \\
4 & \text { Amérique sept. } \\
4 & \text { Europe. }
\end{array}
$$




\begin{tabular}{|c|c|c|c|c|c|}
\hline ESPECES. & 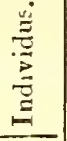 & HABITAT. & ESPËCES. & 点 & HABITAT. \\
\hline Cristatus. & 6 & Europe. & Franklinii. & 1 & Amėrique sept. \\
\hline Dominicus. & 3 & Amérique mér. & Fuscus. & 5 & Brésil. \\
\hline Leucopterus. & 2 & Brésil. & Glaucus. & 2 & Europe. \\
\hline Minor. & 9 & Europe. & Ichthyœtus. & 1 & Afrique. \\
\hline Rolland. & 7 & Malouines. & Leucophthalmus. & 1 & Incie. \\
\hline Rubricollis. & 4 & Europe. & Leucopterus. & 5 & Europe. \\
\hline Espéce indéterminée. & 4 & Nouv.-Hollande. & Marinus. & 6 & loid. \\
\hline \multirow{2}{*}{\multicolumn{3}{|c|}{ GENRE SPEENISCUS. }} & Melanocephalus. & 2 & Ibid. \\
\hline & & & Michaelis. & 1 & Afrique. \\
\hline Demersus. & 4 & Malouines. & Minulus. & 1 & Europe. \\
\hline Minor. & 1 & Nouv.-Hollande. & Nigratoides." & 1 & Chine. \\
\hline Espéce indéterminée. & 1 & Ibid. & Ridibundus. & 4 & Europe. \\
\hline & & & Tenuirostris. & 1 & Inde. \\
\hline GENRE & RI & A. & Tridactylus. & 3 & Europe. \\
\hline
\end{tabular}

\begin{tabular}{l|c|l} 
Alle. & 4 & Europe. \\
Anliqua. & 1 & Amér. borèale. \\
Grylle. & כ̌ & Europe. \\
Troile. & 1 & Ibid. \\
Espèce indéterminée. & 2 &
\end{tabular}

\section{PALMIPÈDES LONGIPENNES.}

\section{GENRE DIOIMEDEA.}

Brachyura.

Chlororhynchos.

Exulans.

Fuliginosa.

Melanophris.

Espéce indéterminée.

\begin{tabular}{|c|l|}
2 & Mers antarctiq. \\
1 & Ibid. \\
2 & Afrique. \\
1 & Mers australes. \\
2 & Afrique. \\
1 &
\end{tabular}

\section{GENRE FALADROMA.}

Bercirdi.

$$
15 \text { |Chili. }
$$

\section{GENRE IARUS.}

Argenlalus.

Atricilla.

Audouinii.

Canus.

Capensis.

Capistratus.

Cirrocephalus.

Eburneus.
Plus, 12 individus formant 8 espèces indéterminées ou inédites provenant de diverses localités.

\section{GENRE IESTIRIS.}

Calaracles.

Parasiticus.

Pomarinus.

Richardsonii.

\begin{tabular}{l|l}
3 & Europe. \\
4 & Ibid. \\
2 & Ibid. \\
8 & Ibid.
\end{tabular}

GENRE PACIITTIIA.

Villata.

| 1 Inde.

\section{GENRE PROGELIARIA.}

Brasiliana.

Capensis.

Desolata.

Gigantea.

Glacialis.

Glacialoides.

Orientalis.

Turtur.

Espèce indéterminée.

Espéce indéterminée.

\begin{tabular}{|l|l}
1 & Brésil. \\
2 & Afrique. \\
1 & Ile de,la Désolat. \\
2 & Océan arclique. \\
3 & Europe, etc. \\
2 & Mers du Nord. \\
1 & Ibid. \\
2 & Mers australes. \\
1 & \\
1 &
\end{tabular}

\section{GENRE PUTEITUS.}

Equinoctialis.

Chlororhynchus.

2 Mers du Sud.

Cinereus.
2 Bourbon.

2 Europe. 


\begin{tabular}{|c|c|c|}
\hline ESPĖCES. & 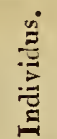 & HABITAT. \\
\hline Curiculus. & 2 & Europe. \\
\hline Major. & 2 & Ibid. \\
\hline Manks. & 1 & Mers du Nord. \\
\hline Obscurus. & 1 & Europe. \\
\hline
\end{tabular}

GeNRE Rey y CeOPS.

Flavirostris.

Nigra.

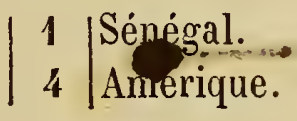

GeNBe STIRNA.

Affinis.

Alba.

Albigena.

Anglica.

Arclica.

Argentea.

Cantiaca.

Caspia.

Dougalli.

Fuliginosa.

Hirundo.

Inca.

Leucopareia.

Leucoptera.

Magnirostris.

Melanauchen.

Melanogaster.

Minuta.

Nigra.

Stolida.

Tenuirostris.

Velox.

\begin{tabular}{|l|l|}
1 & Inde. \\
2 & Ibid. \\
$\mathbf{1}$ & Mer Rouge. \\
$\mathbf{2}$ & Europe \\
$\mathbf{2}$ & Ibid. \\
$\mathbf{2}$ & Orénoque. \\
$\mathbf{5}$ & Europe. \\
$\mathbf{2}$ & Ibid. \\
$\mathbf{4}$ & Ibid. \\
$\mathbf{4}$ & Amérique sept. \\
$\mathbf{4}$ & Europe. \\
$\mathbf{1}$ & Chili. \\
$\mathbf{5}$ & Europe, etc. \\
$\mathbf{7}$ & Ybid. \\
$\mathbf{3}$ & Brésil. \\
$\mathbf{2}$ & Célėbes. \\
$\mathbf{2}$ & Java. \\
$\mathbf{2}$ & Europe. \\
$\mathbf{4}$ & Ibid. \\
$\mathbf{3}$ & Mers du Sud. \\
$\mathbf{2}$ & Sénégal. \\
$\mathbf{3}$ & Afrique. \\
&
\end{tabular}

Plus, 15 individus formant 12 espéces indéterminées ou inédites de diverses localités.

\section{GenRe tratassidroma.}

\begin{tabular}{l|l|l||} 
Columbina. & 2 & Canaries. \\
\hline Fregatta. & 1 & Inde. \\
Leachii. & 2 & Europe. \\
Pelagica. & $\mathbf{2}$ & Ibid. \\
Wilsonii. & 2 & Inde. \\
Espéce indéterminée. & 1 & Nouv.-Zélande.
\end{tabular}

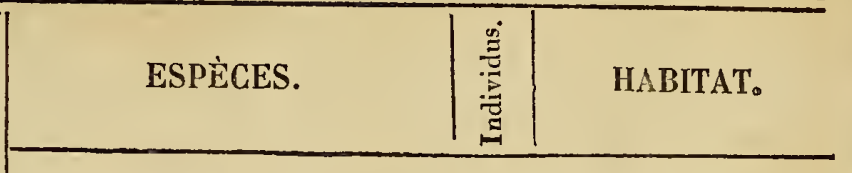 \\ PALMIPÈDES TOTIPALMMES.}

\section{genre cariso.}

Africanus.

Bicristatus.

4 Afrique.

Bougainvillii.

Brasiliensis.

Capensis.

Cormoranus.

Cristatus.

Dimidiatus.

Gaimardi.

Javanicus.

Melanogaster.

Penicillatus.

Punctatus.

Tenuirosiris.

Espèce indéterminée.

Espèce indéterminée.

genRe peLgcandos.

Conspicillalus.

Crispus.

Onocrotalus.

$$
\mid \begin{array}{l|l}
1 & \text { Nouv.-Hollande. } \\
2 & \text { Europe. } \\
4 & \text { Ibid. }
\end{array}
$$

Plus, 5 individus formant $\mathbf{3}$ espéces indéterminées ou inédites de diverses localités.

\section{gente pataton.}
Athereus.
Phœnicurus.

\section{Océan Atlantiq. 2 Océan Pacifique.}

\section{GenRe plotus.}

\begin{tabular}{l|r|l} 
Anhinga. & 4 & Amérique. \\
Levaillantii. & 4 & Afrique. \\
Espéce indéterminée. & 1 & Cochinchine.
\end{tabular}

\section{GenRe SUtA.}

Alba.

Erythrorhyncha.

Fulica.

Espèce indéterminée.

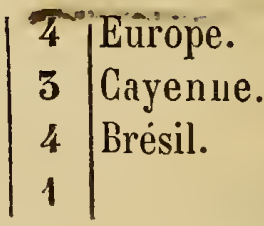




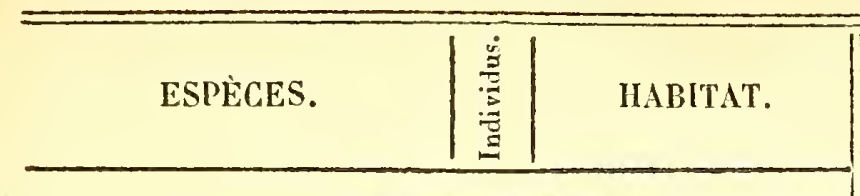

GENRE TACHYPETES.

Aquila.

Espéce indéterminée. Espẻce indéterminée.
2 Océan.

1 Himalaya.

1 Inde.

\section{PALMIPÈDES LAMELLIROSTRES.}

\section{GENRE AINAS.}

Acuta.

Egyptiaca.

Albellus.

Albeola.

Albifrons.

Analoides.

Arcuala.

Alrala.

Autumnalis.

Bernicla.

Bewickii.

Boschas.

Brasiliensis.

\section{Brevipennis.}

Brunnea.

Cana.

Canadensis.

Capensis.

Chiloensis.

Clangula.

Clypeala.

Coromandeliana.

Coscoroba.

Crecca.

Cyanoptera.

Cygnus.

Discors.

Dominica.

Erismalura.

Erythrorhyncha.

Erythrortiyncha.

Falcaria.

Ferina.

Ferruginea.

\begin{tabular}{|c|c|c|}
\hline 6 & Afrique. & Indica. \\
\hline 2 & Egypte. & Jensen. \\
\hline 4 & Europe. & Jubata. \\
\hline 2 & Amérique sept. & Leucocephala. \\
\hline 2 & Europe. & Leucophlhalma. \\
\hline 1 & Brésil. & Leucopsis. \\
\hline 4 & Java. & Lobala. \\
\hline 1 & Nouv.-Hollande. & Maccoa. \\
\hline 5 & Amérique mél. & Maculata. \\
\hline 2 & Europe. & Maculirostris. \\
\hline 1 & Ibid. & Madagascariensis. \\
\hline 2 & Ibid. & Magellanica. \\
\hline 2 & Brésil. & Marila. \\
\hline 3 & Afrique. & Marmoralau \\
\hline 3 & Ibid. & Melanotos. \\
\hline 1 & Ibid. & Membranacea. \\
\hline 2 & Amérique sept. & Merganser. \\
\hline 3 & Afrique. & Mollissima. \\
\hline 5 & Chiloé. & Moschata. \\
\hline 4 & Europe. & Nigra. \\
\hline Ј & Ibid. & Nigriceps. \\
\hline 3 & Coromandel. & Nigricollis. \\
\hline 1 & Amérique mér. & Obscura. \\
\hline 2 & Europe. & Ocyplera. \\
\hline 1 & Abyssinie. & Olor. \\
\hline 2 & Europe. & Oxyura. \\
\hline 6 & Amerique. & Penelope. \\
\hline 2 & Ibid. & Perspicillala. \\
\hline 3 & Afrique. & Pocilorhyncha. \\
\hline 5 & Amérique mér. & Querquedula. \\
\hline 2 & Afrique. & Radjah. \\
\hline $\mathbf{3}$ & Japon. & RafResii. \\
\hline 4 & Europe. & Regia. \\
\hline$T$ & Amérique mér. & Rhynchotis. \\
\hline
\end{tabular}

ellanica.

HABITAT.

Flavirosiris.

Formosa.

Fuligula.

Fulva.

Fusca.

Galericulala.

Gambensis.

Gibbifrons.

Glacialis.

Histrionica.

Hyperborea.

indica.

Jubata.

Leucocephala.

Leucopsis.

Maculata.

Maculirostris

Madagascariensis.

Melanotos

Mollissima.

chata.

Nigricollis

Ocyplera.

Penelope.

Perspicillala.

Radjah.

Raflesiz.

Rhynchotis.
Afrique.

Japon.

Europe.

3 Amérique.

Europe.

3 Chine.

3 Afrique.

2 Célèbes.

Europe.

Ibid.

3 Mexique.

Inde.

3 Tanger.

3 Nouv.-Hollande.

2 Europe.

2 Ibid.

2 Ibid.

2 Nouv.-Hollande.

2 Inde.

2 Amérique mér.

6 Ibid.

4 Madagascar.

5 Amérique mér.

1 Europe.

2 Ibid.

2 Ceylan.

2 Nouv.-Hollande.

6 Europe.

4 Ibid.

4 Ibid.

4 Ibid.

2 Afrique.

1 Amérique mér.

5 Amérique sept.

1 Ibid.

6 Europe.

3 Amérique mér.

7 Europe.

1 Ibid.

3 Ceylan.

$\because$ Europe, etc.

1 Australie.

4 Amérique mér.

6 Brésil.

5 Nouv.-Hollande. 


\begin{tabular}{|c|c|c|c|c|c|}
\hline ESPĖCES. & 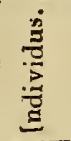 & HABITAT. & ESPÈCES. & 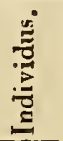 & HABITAT. \\
\hline $\begin{array}{l}\text { Rubida. } \\
\text { Rufina. } \\
\text { Rutila. } \\
\text { Segetum. } \\
\text { Serrator. } \\
\text { Smithii. } \\
\text { Speclabilis. } \\
\text { Spinosa. } \\
\text { Sponsa. }\end{array}$ & $\begin{array}{l}3 \\
3 \\
3 \\
5 \\
2 \\
1 \\
4 \\
1 \\
3\end{array}$ & $\begin{array}{l}\text { Amérique mér. } \\
\text { Europe. } \\
\text { Tanger. } \\
\text { Europe. } \\
\text { Ibid. } \\
\text { Afrique. } \\
\text { Europe. } \\
\text { Amérique mér. } \\
\text { Ibid. }\end{array}$ & $\begin{array}{l}\text { Strepera. } \\
\text { Superciliosa. } \\
\text { Tadorna. } \\
\text { Tadornoides. } \\
\text { Torquata. } \\
\text { Urophasianus. } \\
\text { Valisneria. } \\
\text { Viduata. }\end{array}$ & $\begin{array}{l}3 \\
2 \\
2 \\
2 \\
5 \\
2 \\
3 \\
5\end{array}$ & $\begin{array}{l}\text { Europe. } \\
\text { Nouv.-Hollande. } \\
\text { Europe. } \\
\text { Nouv.-Hollande. } \\
\text { Amérique mér. } \\
\text { lbid. } \\
\text { Amérique sept. } \\
\text { Amérique mér. }\end{array}$ \\
\hline
\end{tabular}

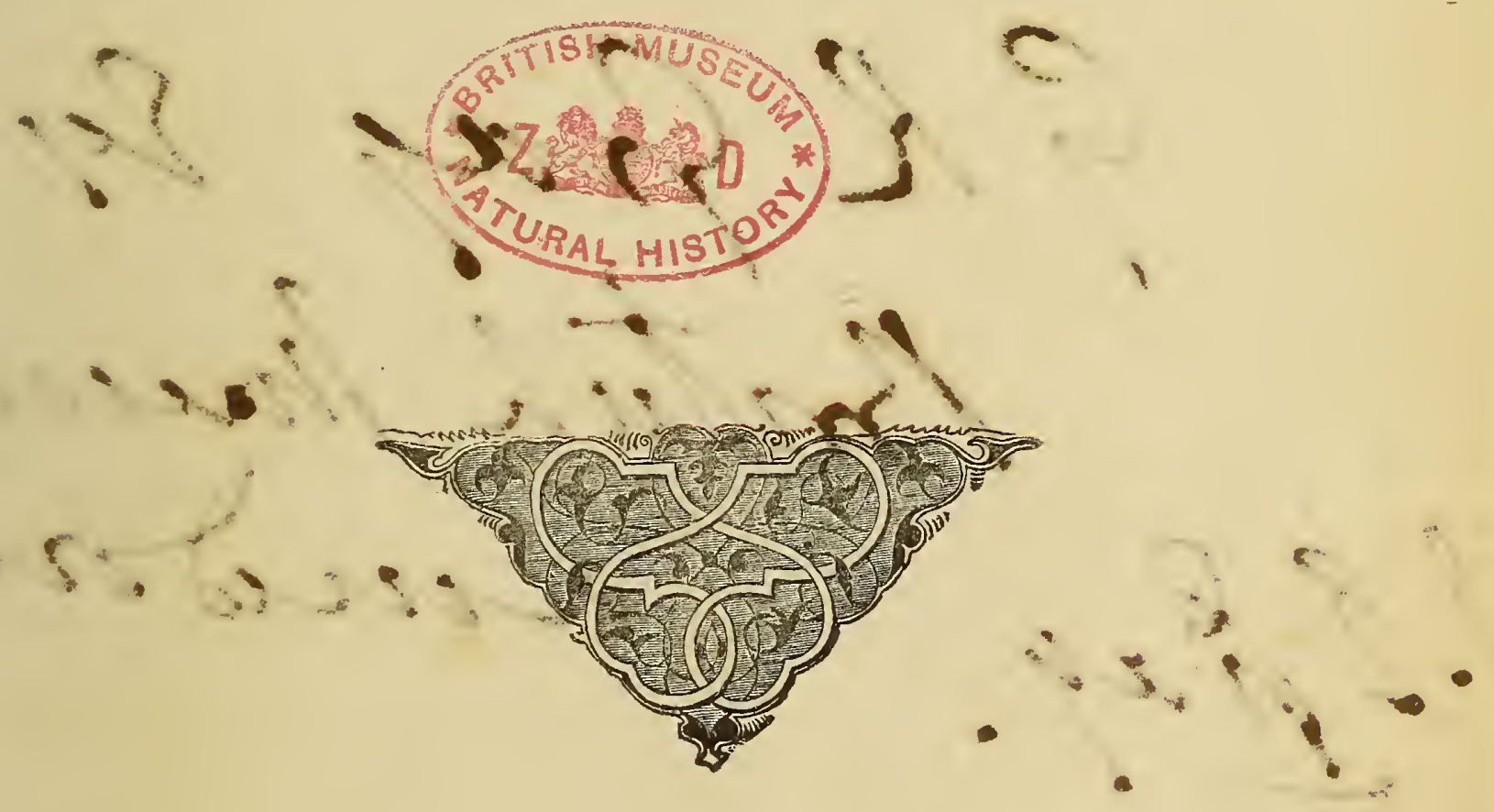


GR Gay Sy Bntuki inuceen 2\% Liniton 2 fray. 

